\title{
Discovery of (1H-Pyrazolo[3,4-c]pyridin-5- yl)sulfonamide Analogs as Hepatitis B Virus Capsid Assembly Modulators by Conformation Constraint
}

Chunting Wang ${ }^{1, \#}$, Yameng Peil, ${ }^{1, \#}$ Lin Wang ${ }^{3}$, Shuo Li ${ }^{4}$, Chao Jiang ${ }^{4}, X u$ Tan $^{4}, Y i$

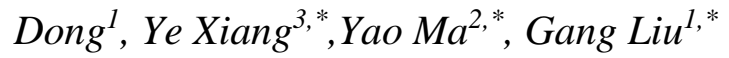

1, School of Pharmaceutical Sciences, Tsinghua University, Beijing 100084, China.

2, Institute of Materia Medica, Chinese Academy of Medical Sciences \& Peking Union Medical College, Beijing 100050, China.

3, Beijing Advanced Innovation Center for Structural Biology, Collaborative Innovation Center for Diagnosis and Treatment of Infectious Diseases, Center for Global Health and Infectious Diseases, Department of Basic Medical Sciences, School of Medicine, Tsinghua University, Beijing 100084, China.

4, Beijing Advanced Innovation Center for Structural Biology, Beijing Frontier Research Center for Biological Structure, School of Pharmaceutical Sciences, Center for Infectious Disease Research, School of Medicine, Tsinghua University, TsinghuaPeking Center for Life Sciences, Beijing 100084, China.

\section{Contents:}

1) Supplementary Table S1. Anti-HBV activity and cytotoxicity of compounds with various substituents at the sulfonamide moiety.

2) Supplementary Figure S1. In vivo anti-HBV activity of 56 and $\mathbf{6 7}$ (i.v. qod. and p.o. qd.) in HDI mouse model.

3) Supplementary Figure S2. In vivo anti-HBV activity of 56 and 67 (p.o. bid.) in HDI mouse model. 
4) Supplementary Figure S3. Inhibition of intracellular HBV RNA in HBV infected Huh7-NTCP

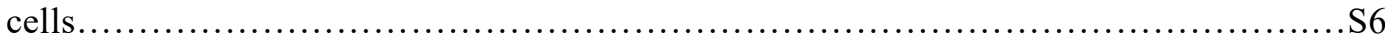

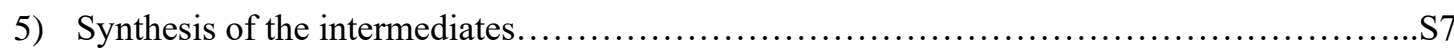

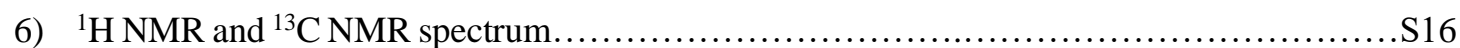

7) Supplementary Table S2. Purity of the tested compounds................................ 84

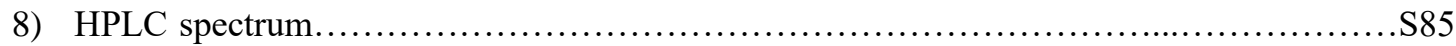


Supplementary Table S1: Anti-HBV activity and cytotoxicity in HepAD38 cells of compounds with various substituents at the sulfonamide moiety.<smiles>[R5]S(=O)(=O)c1ccc2c(c1)c(Nc1ccc(F)c(C)c1)nn2C1CC1</smiles>

\begin{tabular}{|c|c|c|c|}
\hline Compd. & $\mathrm{R}_{3}$ & $\mathrm{IC}_{50}(\mu \mathrm{M})$ & $\mathrm{CC}_{50}(\mu \mathrm{M})$ \\
\hline S1 & & 0.17 & 23.23 \\
\hline S2 & & 0.083 & 13.5 \\
\hline S3 & & 0.22 & 14.06 \\
\hline S4 & & 0.21 & 19.34 \\
\hline S5 & & 0.23 & $>100$ \\
\hline S6 & & 0.070 & 8.85 \\
\hline S7 & & 0.079 & 27.8 \\
\hline
\end{tabular}




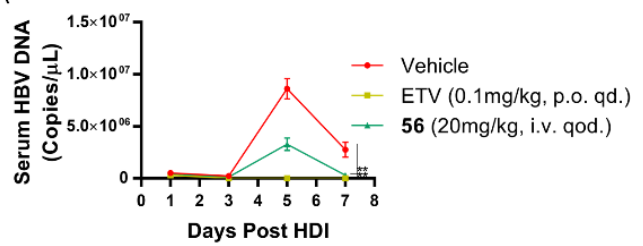

B

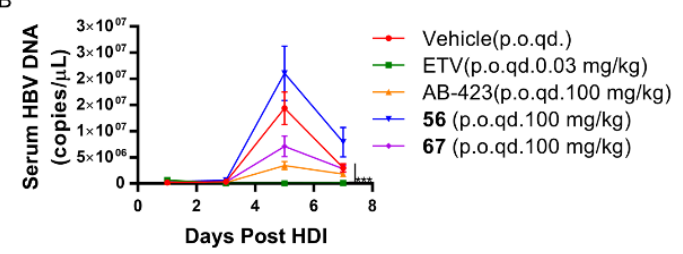

Supplementary Figure S1. In vivo anti-HBV activity of 56 and $\mathbf{6 7}$ in HDI mouse model. (A) In vivo anti-HBV activity of $\mathbf{5 6}$ administered intravascularly. (B) In vivo anti-HBV activity of $\mathbf{5 6}$ and $\mathbf{6 7 .}$ Results are presented as mean \pm SEM. $N=7 ; * *, \mathrm{P}<0.01 ; * * *, \mathrm{P}<0.001$. 

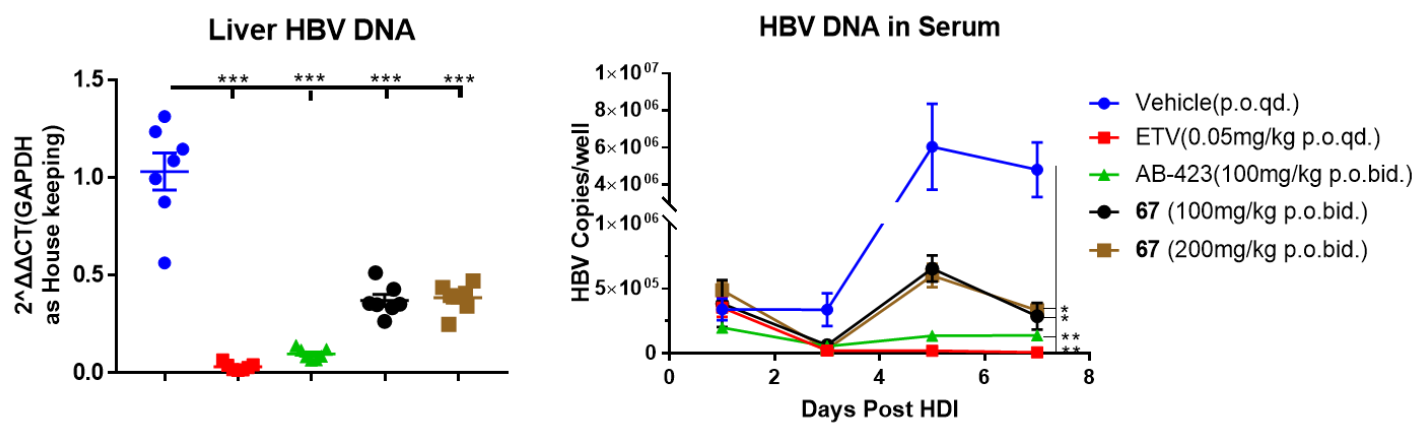

Supplementary Figure S2. In vivo anti-HBV activity of $\mathbf{5 6}$ and 57 (p.o. bid.) in HDI mouse model. Results are presented as mean \pm SEM. $N=7 ; *, \mathrm{P}<0.05 ; * *, \mathrm{P}<0.01 ; * * *, \mathrm{P}<0.001$. 


\section{Intracellular RNA Level in HBV Infected Huh7-NTCP cells}

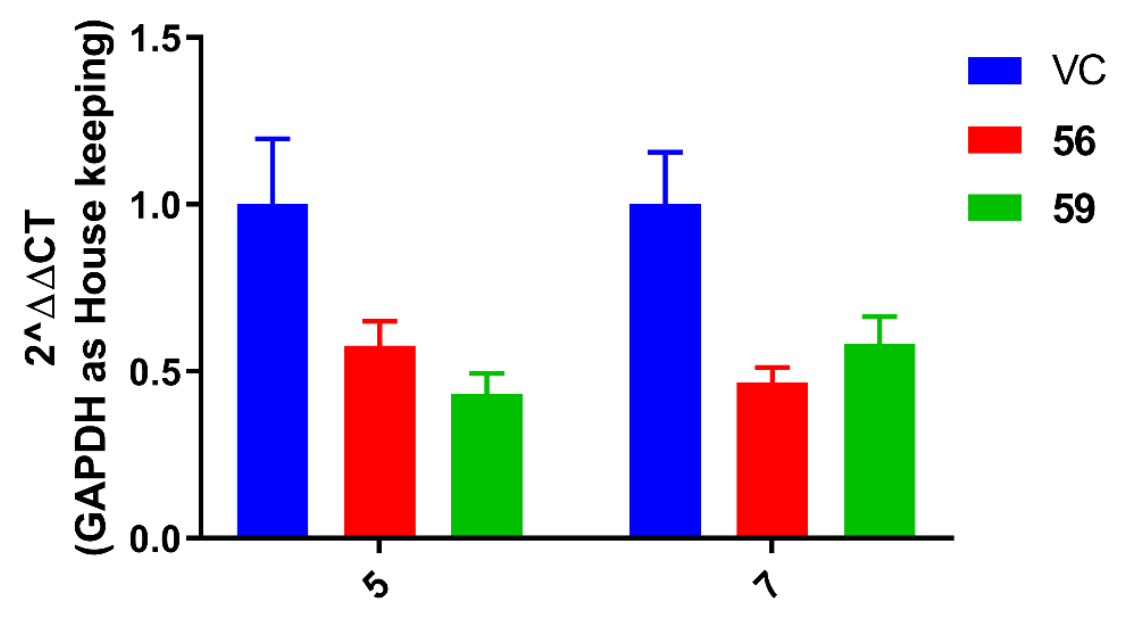

Days Post Infection

Supplementary Figure S3. Inhibition of intracellular HBV RNA in HBV infected Huh7-NTCP cells. The Huh7-NTCP cells $\left(2 \times 10^{5}\right.$ cells/well in 24 micro-well plate) were infected by HBV (200MOI) for $24 \mathrm{~h}$, and then treated with $1.0 \mu \mathrm{M}$ compounds or DMSO (VC) for 5 or 7 days. Cells were collected and intracellular RNA was extracted and detected by qPCR. The data is presented as mean \pm SD. 


\section{Synthesis of the intermediates}

Synthesis of 72-75.<smiles>Brc1ccccc1</smiles>

5-(benzylthio)-1H-indazole (72). 5-Bromoindazole (390 mg, $2 \mathrm{mmol}), \mathrm{Pd}_{2}(\mathrm{dba})_{3}(91 \mathrm{mg}, 0.1$ mmol), Xantphos (117 mg, $0.2 \mathrm{mmol}$ ) were dissolved in $20 \mathrm{~mL}$ 1,4-dioxane. Then DIPEA (660 $\mu \mathrm{L}$, $4 \mathrm{mmol})$ and benzyl mercaptan $(372 \mathrm{mg}, 3 \mathrm{mmol})$ were added. The mixture was heated at $90{ }^{\circ} \mathrm{C}$ under argon atmosphere for $4 \mathrm{~h}$. After that, the mixture was filtered and the filtrate was concentrated under vacuum. The residue was purified by column chromatography on silica gel (eluted with PE/EA, 2/1) to get $440 \mathrm{mg} 1-2$ as orange oil. Yield: $92 \%$.<smiles>O=S(=O)(c1ccc2[nH]ncc2c1)N1CCC(O)CC1</smiles>

1-((1H-indazol-5-yl)sulfonyl)piperidin-4-ol (73). $420 \mathrm{mg}(1.75 \mathrm{mmol}) \mathbf{7 2}$ was dissolved in mixture of $10 \mathrm{~mL}$ acetonitrile, $100 \mu \mathrm{L} \mathrm{H}_{2} \mathrm{O}$ and $100 \mu \mathrm{L} \mathrm{AcOH}$. After the mixture was cooled to $-30{ }^{\circ} \mathrm{C}, 5,5-$ dimethylhydantoin $(520 \mathrm{mg}, 2.62 \mathrm{mmol}$ ) was added portion wise. Then the mixture was warmed to $-15{ }^{\circ} \mathrm{C}$ and stirred for another hour. The mixture was diluted with EA and washed with brine. The organic layer was dried using anhydrous sodium sulfate. EA was removed under vacuum. The residue was dissolved with DCM. 4-hydroxylpiperidine (520 mg, $5.2 \mathrm{mmol})$ and TEA (1.0 ml, 7.86 $\mathrm{mmol}$ ) were added. The mixture was stirred at room temperature for $30 \mathrm{mins}$ and water was added. The organic layer was separated, washed with brine and dried using anhydrous sodium sulfate. DCM was evaporated under vacuum. The residue was then purified using flash chromatography on $\mathrm{C}_{18}$ silica gel (eluted with water/methanol) to give $200 \mathrm{mg} 73$ as white powder. Yield $41 \%$.<smiles>O=S(=O)(c1ccc2[nH]nc(Br)c2c1)N1CCC(O)CC1</smiles>

1-((3-bromo-1H-indazol-5-yl)sulfonyl)piperidin-4-ol (74). To the mixture of 73 (93 $\mathrm{mg}, 0.33 \mathrm{mmol})$ in chloroform was added NBS ( $65 \mathrm{mg}, 0.37 \mathrm{mmol})$. The resulting mixture was stirred at $60^{\circ} \mathrm{C}$. After completion, saturated $\mathrm{Na}_{2} \mathrm{~S}_{2} \mathrm{O}_{3}$ solution was added. The organic phase was separated, washed with brine and dried using anhydrous sodium sulfate. DCM was evaporated under vacuum to give $\mathbf{7 4}$ which was used for next step without purification.<smiles>CC(=O)OC1CCN(S(=O)(=O)c2ccc3c(c2)c(Br)nn3C2CCCCO2)CC1</smiles>

1-((3-bromo-1-(tetrahydro-2H-pyran-2-yl)-1H-indazol-5-yl)sulfonyl)piperidin-4-yl acetate (75). To the solution of 1-4 obtained above in EA was added 3,4-Dihydro-2H-pyran $(55 \mathrm{mg}, 0.66 \mathrm{mmol})$ and $p$-Toluenesulfonic acid monohydrate $(19 \mathrm{mg}, 0.1 \mathrm{mmol})$. The resulting mixture was stirred under reflux for $16 \mathrm{~h} . \mathrm{Ac}_{2} \mathrm{O}(67 \mu \mathrm{L}, 0.66 \mathrm{mmol})$ was then added. After completion, the mixture was washed 
with water, brine and dried by anhydrous sodium sulfate. After evaporation, the residue was then purified using flash chromatography on silica gel (eluted with PE/EA) to give $138 \mathrm{mg} \mathbf{6 5}$ as white powder. Yield $86 \%$.

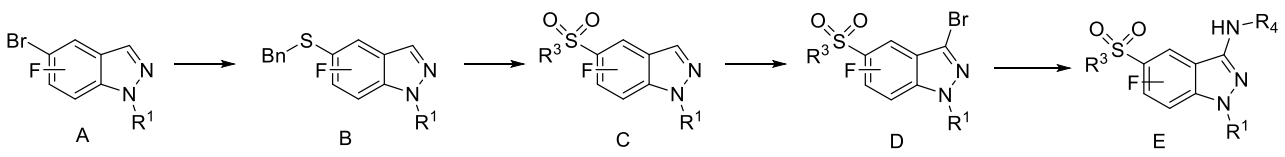

Synthesis of intermediate A.<smiles>Cn1ncc2cc(Br)ccc21</smiles>

5-bromo-1-methyl-1H-indazole. To the solution of 5-bromo- $1 H$-indazole (394 mg, $2 \mathrm{mmol}$ ) in acetone was added $\mathrm{CH}_{3} \mathrm{I}(870 \mathrm{mg}, 6 \mathrm{mmol})$ and $\mathrm{K}_{2} \mathrm{CO}_{3}(552 \mathrm{mg}, 4 \mathrm{mmol})$. The resulting mixture was stirred at $80^{\circ} \mathrm{C}$ for $12 \mathrm{~h}$. After filtration, acetone was evaporated under vacuum. Purification by column chromatography on silico gel (eluted with PE/EA, 2/1) afforded the $220 \mathrm{mg}$ product as white powder. Yield: 59\%. ${ }^{1} \mathrm{H}$ NMR (400 MHz, Chloroform- $d$ ) $\delta 7.95(\mathrm{~d}, J=1.0 \mathrm{~Hz}, 1 \mathrm{H}), 7.89$ (dd, $J=$ $1.8,0.7 \mathrm{~Hz}, 1 \mathrm{H}), 7.49(\mathrm{dd}, J=8.9,1.8 \mathrm{~Hz}, 1 \mathrm{H}), 7.31(\mathrm{dt}, J=8.9,0.9 \mathrm{~Hz}, 1 \mathrm{H}), 4.09$ (s, 3H).

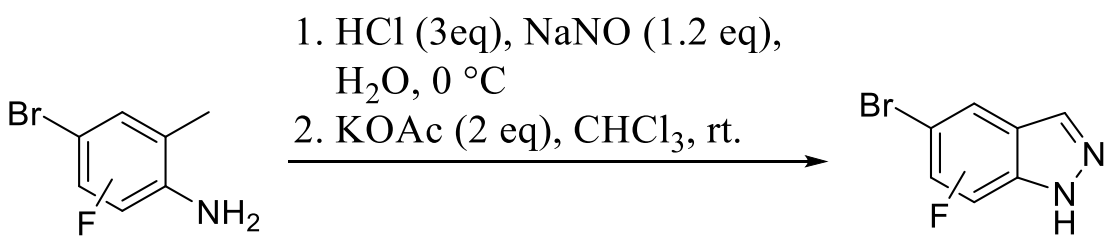

To the mixture of the aniline $(6 \mathrm{mmol})$ in $\mathrm{H}_{2} \mathrm{O}$ and hydrochloric acid $(2.5 \mathrm{~mL})$ at $0{ }^{\circ} \mathrm{C}$ under stirring was added aqueous NaNO (7.2 mmol). After $30 \mathrm{~min}, \mathrm{H}_{2} \mathrm{O}$ was evaporated under vacuum. The residues were dispersed in $\mathrm{CHCl}_{3}$. To the mixture, KOAc $(18 \mathrm{mmol})$ was added. The resulting mixture was stirred at room temperature until completion. $\mathrm{CHCl}_{3}$ was evaporated under vacuum. The residue was washed with water and dried under vacuum to afford the product.<smiles>Brc1ccc2c(cnn2C2CC2)c1</smiles>

5-bromo-1-cyclopropyl-1H-indazole. The mixture of 5-bromo- $1 H$-indazole $(985 \mathrm{mg}, 5 \mathrm{mmol})$, cyclopropylboronic acid ( $859 \mathrm{mg}, 10 \mathrm{mmol}), \mathrm{Cu}(\mathrm{OAc})_{2}(900 \mathrm{mg}, 5 \mathrm{mmol}), 2$,'-Bipyridine (780 mg, $5 \mathrm{mmol})$ and $\mathrm{Na}_{2} \mathrm{CO}_{3}(1.0 \mathrm{~g}, 10 \mathrm{mmol})$ in $30 \mathrm{~mL} \mathrm{1,2-DCE}$ was stirred at $70^{\circ} \mathrm{C}$ for $2 \mathrm{~h}$. After filtration, the filtrate was concentrated under vacuum. The residue was dissolved in EA and washed with saturated aqueous $\mathrm{CuSO}_{4}$, brine and dried using anhydrous sodium sulfate. EA was evaporated under vacuum. The product was used for next step without purification.<smiles>Brc1ccc2c(cnn2C2COC2)c1</smiles>

5-bromo-1-(oxetan-3-yl)-1H-indazole. To the solution of 5-bromo- $1 H$-indazole ( $329 \mathrm{mg}, 1.67 \mathrm{mmol})$ in DMF were added oxetan-3-yl 4-methylbenzenesulfonate (570 mg, $2.5 \mathrm{mmol})$ and $\mathrm{K}_{2} \mathrm{CO}_{3}(691 \mathrm{mg}$, 
$5 \mathrm{mmol})$. The resulting mixture was stirred at $110^{\circ} \mathrm{C}$ for $12 \mathrm{~h}$. After filtration, DMF was evaporated under vacuum. Purification by column chromatography on silico gel (eluted with PE/EA, 4/1) afforded the product.<smiles>CCn1ncc2cc(Br)ccc21</smiles>

5-bromo-1-ethyl-1H-indazole. To the solution of 5-bromo- $1 \mathrm{H}$-indazole (591 $\mathrm{mg}, 3 \mathrm{mmol})$ in DMF were added $\mathrm{CH}_{3} \mathrm{CH}_{2} \mathrm{I}(936 \mathrm{mg}, 6 \mathrm{mmol})$ and $\mathrm{K}_{2} \mathrm{CO}_{3}(1.24 \mathrm{~g}, 9 \mathrm{mmol})$. The resulting mixture was stirred at $40{ }^{\circ} \mathrm{C}$ for $12 \mathrm{~h}$. After filtration, DMF was evaporated under vacuum. Purification by column chromatography on silico gel (eluted with PE/EA, 8/1) afforded $125 \mathrm{mg}$ product. Yield: 19\%. ${ }^{1} \mathrm{H}$ NMR (400 MHz, Chloroform- $d$ ) $\delta 7.94(\mathrm{~d}, J=1.1 \mathrm{~Hz}, 1 \mathrm{H}), 7.88(\mathrm{~d}, J=1.8 \mathrm{~Hz}, 1 \mathrm{H}), 7.46$ (dd, $J=8.9,1.8 \mathrm{~Hz}, 1 \mathrm{H}), 7.31(\mathrm{~d}, J=8.9 \mathrm{~Hz}, 1 \mathrm{H}), 4.43$ (q, $J=7.3 \mathrm{~Hz}, 2 \mathrm{H}), 1.52$ (t, $J=7.3 \mathrm{~Hz}, 3 \mathrm{H})$.<smiles>CC(C)n1ncc2cc(Br)ccc21</smiles>

5-bromo-1-isopropyl-1H-indazole. To the solution of 5-bromo- $1 H$-indazole (296 mg, $1.5 \mathrm{mmol})$ in $10 \mathrm{~mL}$ DMF were added $i$-PrI $(510 \mathrm{mg}, 3 \mathrm{mmol})$ and DBU (456 mg, $3 \mathrm{mmol})$. The mixture was stirred at room temperature for $48 \mathrm{~h}$. After evaporation under vacuum, the residue was purified by column chromatography on silico gel (eluted with PE/EA, 10/1) to afford $160 \mathrm{mg}$ product. Yield: 45\%. ${ }^{1} \mathrm{H}$ NMR (400 MHz, Chloroform- $d$ ) $\delta 7.94$ (s, $1 \mathrm{H}$ ), 7.86 (dd, $J=1.7,0.8 \mathrm{~Hz}, 1 \mathrm{H}$ ), 7.43 (dd, $J$ $=8.9,1.7 \mathrm{~Hz}, 1 \mathrm{H}), 7.33(\mathrm{~d}, J=8.9 \mathrm{~Hz}, 1 \mathrm{H}), 4.81$ (hept, $J=6.7 \mathrm{~Hz}, 1 \mathrm{H}), 1.59$ (d, $J=6.6 \mathrm{~Hz}, 6 \mathrm{H})$.<smiles>Brc1ccc2c(cnn2C2CCC2)c1</smiles>

5-bromo-1-cyclobutyl-1H-indazole. To the solution of 5-bromo- $1 \mathrm{H}$-indazole ( $296 \mathrm{mg}, 1.5 \mathrm{mmol})$ in $10 \mathrm{~mL}$ DMF were added cyclobutyl bromide (405 mg, $3 \mathrm{mmol}$ ) and $\mathrm{NaH}(180 \mathrm{mg}(60 \%), 4.5 \mathrm{mmol})$. The mixture was stirred at $50^{\circ} \mathrm{C}$ for $12 \mathrm{~h}$. After evaporation under vacuum, the residue was purified by column chromatography on silico gel (eluted with PE/EA, 10/1) to afford $170 \mathrm{mg}$ product. Yield: $45 \%$.

\section{Synthesis of intermediate $B$.}

Examples:<smiles>c1ccc(Sc2ccc3c(cnn3C3CC3)c2)cc1</smiles>

5-(benzylthio)-1-cyclopropyl-1H-indazole. To the solution of 5-bromo-1-cyclopropyl-1H-indazole (crude product, $5 \mathrm{mmol}$ ) was added $\mathrm{Pd}_{2}(\mathrm{dba})_{3}(229 \mathrm{mg}, 0.025 \mathrm{mmol}$ ), Xantphos ( $289 \mathrm{mg}, 0.5 \mathrm{mmol}$ ), DIPEA $(1650 \mu \mathrm{L}, 10 \mathrm{mmol})$ and benzyl mercaptan $(930 \mathrm{mg}, 7.5 \mathrm{mmol})$. The resulting mixture was stirred at $90{ }^{\circ} \mathrm{C}$ for $4 \mathrm{~h}$. After filtration and evaporation, the residue was purified by column chromatography on silico gel (eluted with PE/EA, 8/1) to afford $854 \mathrm{mg}$ product as yellow solid. 
Yield: $61 \%$ for 2 steps. ${ }^{1} \mathrm{H}$ NMR (400 MHz, Chloroform- $d$ ) $\delta 7.84(\mathrm{~d}, J=0.9 \mathrm{~Hz}, 1 \mathrm{H}), 7.66(\mathrm{dd}, J$ $=1.7,0.8 \mathrm{~Hz}, 1 \mathrm{H}), 7.48(\mathrm{dt}, J=8.7,0.9 \mathrm{~Hz}, 1 \mathrm{H}), 7.35(\mathrm{dd}, J=8.7,1.6 \mathrm{~Hz}, 1 \mathrm{H}), 7.28-7.16(\mathrm{~m}$, $5 \mathrm{H}), 4.06(\mathrm{~s}, 2 \mathrm{H}), 3.56(\mathrm{tt}, J=7.1,3.7 \mathrm{~Hz}, 1 \mathrm{H}), 1.24-1.18(\mathrm{~m}, 2 \mathrm{H}), 1.18-1.10(\mathrm{~m}, 2 \mathrm{H})$.

\section{Synthesis of intermediate $C$}

Example:<smiles>CC(=O)OC1CCN(S(=O)(=O)c2ccc3c(cnn3C3CC3)c2)CC1</smiles>

1-((3-bromo-1-cyclopropyl-1H-indazol-5-yl)sulfonyl)piperidin-4-yl acetate. To the solution of 5(benzylthio)-1-cyclopropyl-1 $H$-indazole ( $140 \mathrm{mg}, 0.5 \mathrm{mmol})$ in $5 \mathrm{~mL} \mathrm{CH}_{3} \mathrm{CN}, 100 \mu \mathrm{L} \mathrm{H} 2 \mathrm{O}$ and 100 $\mu \mathrm{L} \mathrm{AcOH}$ was added 5,5-dimethylhydantoin $(200 \mathrm{mg}, 1.0 \mathrm{mmol})$ slowly at $-15^{\circ} \mathrm{C}$ under stirring. The mixture was kept at $-15^{\circ} \mathrm{C}$ for $1 \mathrm{~h}$. EA was added; the organic phase was washed with brine and dried by anhydrous sodium sulfate. EA was evaporated under vacuum. To the solution of the above product in DCM was added 4-hydroxylpiperidine (100 mg, $1 \mathrm{mmol})$ and TEA (277 $\mu \mathrm{L}, 1$ $\mathrm{mmol})$. The mixture was stirred at room temperature for $30 \mathrm{~min}$. Then it was washed with water and brine, and dried using anhydrous sodium sulfate. DCM was evaporated under vacuum. To the solution of the above product in EA was added $\mathrm{Ac}_{2} \mathrm{O}(600 \mu \mathrm{L}, 5 \mathrm{mmol})$ and pyridine $(160 \mu \mathrm{L}, 2$ mmol). The mixture was stirred at reflux until disappearance of the substrate. The organic phase was washed with saturated aqueous $\mathrm{CuSO}_{4}$, brine and dried using anhydrous sodium sulfate. EA was evaporated under vacuum to afford the product, which can be used for next step without further purification.

Synthesis of intermediate D

Examples:<smiles>CC(=O)OC1CCN(S(=O)(=O)c2ccc3c(c2)c(Br)nn3C2CC2)CC1</smiles>

To the solution of the substrate (crude product, $0.5 \mathrm{mmol}$ ) in the mixture of $4 \mathrm{~mL} \mathrm{CH}_{3} \mathrm{CN}$ and $2 \mathrm{~mL}$ AcOH was added NBS $(133 \mathrm{mg}, 0.75 \mathrm{mmol})$ at room temperature under stirring. The reaction was monitored using LC-MS. Upon the disappearance of the substrate, EA was added. The organic phase was washed with saturated aqueous $\mathrm{NaHCO}_{3}$ and brine. After evaporation, the residue was purified by column chromatography on silico gel (eluted with PE/EA, 8/1) to afford $140 \mathrm{mg}$ product as white powder. Yield: 64\%. ${ }^{1} \mathrm{H}$ NMR (400 MHz, DMSO- $\left.d_{6}\right) \delta 8.00(\mathrm{~d}, J=8.9 \mathrm{~Hz}, 1 \mathrm{H}), 7.94(\mathrm{~s}, 1 \mathrm{H}), 7.85$ $(\mathrm{d}, J=9.0 \mathrm{~Hz}, 1 \mathrm{H}), 4.67$ (s, 1H), 3.89 (p, $J=5.2 \mathrm{~Hz}, 1 \mathrm{H}), 3.31-3.12(\mathrm{~m}, 3 \mathrm{H}), 2.87$ (d, $J=10.3 \mathrm{~Hz}$, 2H), $2.02-1.80(\mathrm{~m}, 5 \mathrm{H}), 1.61(\mathrm{q}, J=9.9 \mathrm{~Hz}, 2 \mathrm{H}), 1.19(\mathrm{~d}, J=8.1 \mathrm{~Hz}, 4 \mathrm{H})$. 
<smiles>CC(=O)OC1CCN(S(=O)(=O)c2ccc3c(cnn3C3CC3)c2F)CC1</smiles><smiles>CC(=O)OCC(C)(C)C(=O)O</smiles><smiles>CC(=O)OC1CCN(S(=O)(=O)c2ccc3c(c(Br)nn3C3CC3)c2F)CC1</smiles>

To the solution of the substrate $(76 \mathrm{mg}, 0.2 \mathrm{mmol}$ ) in $3 \mathrm{~mL} \mathrm{AcOH}$ was added dibromo-5,5dimethylhydantoin ( $86 \mathrm{mg}, 0.3 \mathrm{mmol}$ ) at $40{ }^{\circ} \mathrm{C}$ under stirring. Upon the disappearance of the substrate, $\mathrm{AcOH}$ was evaporated under vacuum. The residue was purified with thin layer chromatography on silico gel (eluted with PE/EA, 2/1) to afford the $64 \mathrm{mg}$ product. Yield: 70\%.<smiles>CC(=O)OC1CCN(S(=O)(=O)c2cc3cnn(C4CC4)c3cc2F)CC1</smiles>

To the solution of the substrate ( $350 \mathrm{mg}, 0.92 \mathrm{mmol}$ ) in $10 \mathrm{~mL} \mathrm{AcOH}$ was added NBS (481 mg, 2.7 mmol) at $40{ }^{\circ} \mathrm{C}$ under stirring. Upon the disappearance of the substrate, $\mathrm{AcOH}$ was evaporated under vacuum. The residue was purified with column chromatography on silico gel (eluted with PE/EA, $3 / 1$ ) to afford the $180 \mathrm{mg}$ product. Yield: $42 \%$.<smiles>CC(=O)OC1CCN(S(=O)(=O)c2cc(F)c3c(cnn3C3CC3)c2)CC1</smiles>

To the solution of the substrate $(133 \mathrm{mg}, 0.35 \mathrm{mmol})$ in $10 \mathrm{~mL} \mathrm{AcOH}$ was added NBS (187 mg, $1.05 \mathrm{mmol}$ ) at $40{ }^{\circ} \mathrm{C}$ under stirring. Upon the disappearance of the substrate, $\mathrm{AcOH}$ was evaporated under vacuum. The residue was purified with thin layer chromatography on silico gel (eluted with PE/EA, 4/1) to afford the $45 \mathrm{mg}$ product. Yield: $28 \%$.

Synthesis of 78-84.<smiles>Cc1cc(Br)ncc1N</smiles>

6-bromo-4-methylpyridin-3-amine (78). To the solution of 2-Bromo-5-nitro-4-picoline (2.0 g, 9.3 mmol) in methanol was added Raney Ni. The resulting mixture was stirred at room temperature under hydrogen atmosphere for $48 \mathrm{~h}$. Raney $\mathrm{Ni}$ was removed through filtration; the filtrate was evaporated to afford 78, which was used for next step without purification.<smiles>Cc1cc(Br)ncc1NC(Cl)Cl</smiles>

$\mathrm{N}$-(6-bromo-4-methylpyridin-3-yl)acetamide (79). The mixture of 78 (crude product, $9.3 \mathrm{mmol}$ ) and $\mathrm{Ac}_{2} \mathrm{O}(2.86 \mathrm{~mL}, 28 \mathrm{mmol})$ in $\mathrm{PhCH}_{3}$ was stirred at $100{ }^{\circ} \mathrm{C}$ for $2 \mathrm{~h}$. $\mathrm{PhCH}_{3}$ was evaporated under 
vacuum. The residue was purified with column chromatography on silico gel to afford $1.7 \mathrm{~g}$ compound 79 as white powder. Yield: $85 \%$ for 2 steps.<smiles>Brc1cc2cn[nH]c2cn1</smiles>

5-bromo-1H-pyrazolo[3,4-c]pyridine (80). To the solution of 79 in $20 \mathrm{~mL} \mathrm{PhCH}_{3}$ was added isoamyl nitrite (2.34 g, $20 \mathrm{mmol}), \mathrm{Ac}_{2} \mathrm{O}(1.6 \mathrm{~mL}, 16 \mathrm{mmol}), \mathrm{KOAc}(1.44 \mathrm{~g}, 16 \mathrm{mmol})$ and 18 crown-6 (211 mmol, $16 \mathrm{mmol})$. The resulting mixture was stirred at $80^{\circ} \mathrm{C}$ for $20 \mathrm{~h}$. $\mathrm{PhCH}_{3}$ was evaporated and the residue was dissolved in EA. The organic phase was washed with water and brine. EA was evaporated under vacuum. The residue was purified with column chromatography on silico gel. The product obtained above was dissolved in THF/water $(10 / 10 \mathrm{~mL})$. NaOH $(1.28 \mathrm{~g}, 32$ mmol) was added. The resulting mixture was stirred at room temperature for $2 \mathrm{~h}$. After evaporating the THF under vacuum, $1 \mathrm{~N} \mathrm{HCl}$ was added slowly to the aqueous phase to make the $\mathrm{pH} \approx 7$. The solid was collected through filtration and dried under vacuum. Yield: $1.03 \mathrm{~g}, 63 \%$. ${ }^{1} \mathrm{H}$ NMR (400 MHz, DMSO- $\left.d_{6}\right) \delta 13.83(\mathrm{~s}, 1 \mathrm{H}), 8.88(\mathrm{~s}, 1 \mathrm{H}), 8.21(\mathrm{~d}, J=1.2 \mathrm{~Hz}, 1 \mathrm{H}), 8.04(\mathrm{~d}, J=1.0 \mathrm{~Hz}, 1 \mathrm{H})$. $\mathrm{BnS}$<smiles>Cc1cc2cn[nH]c2cn1</smiles>

5-(benzylthio)-1H-pyrazolo[3,4-c]pyridine (81). To the solution of 80 (1.0 g, $5 \mathrm{mmol}$ ) was added $\mathrm{Pd}(\mathrm{dba})_{2}(144 \mathrm{mg}, 0.25 \mathrm{mmol})$, Xantphos (144 mg, $\left.0.25 \mathrm{mmol}\right)$, DIPEA $(1650 \mu \mathrm{L}, 10 \mathrm{mmol})$ and benzyl mercaptan $(930 \mathrm{mg}, 7.5 \mathrm{mmol})$. The resulting mixture was stirred at $90{ }^{\circ} \mathrm{C}$ for $4 \mathrm{~h}$. After filtration and evaporation, the residue was purified by column chromatography on silico gel (eluted with PE/EA, 2/1) to afford $1.22 \mathrm{~g}$ product as yellow solid. Yield: $87 \%$. ${ }^{1} \mathrm{H}$ NMR (400 MHz, Chloroform- $d$ ) $\delta 10.62(\mathrm{~s}, 1 \mathrm{H}), 9.00(\mathrm{~s}, 1 \mathrm{H}), 8.02(\mathrm{~s}, 1 \mathrm{H}), 7.57(\mathrm{~d}, J=1.2 \mathrm{~Hz}, 1 \mathrm{H}), 7.39-7.32(\mathrm{~m}$, $2 \mathrm{H}), 7.29-7.24(\mathrm{~m}, 2 \mathrm{H}), 7.23-7.18(\mathrm{~m}, 1 \mathrm{H}), 4.42(\mathrm{~s}, 2 \mathrm{H})$.<smiles>Br[Sb]c1cc2cnn(C3CC3)c2cn1</smiles>

5-(benzylthio)-1-cyclopropyl-1H-pyrazolo[3,4-c]pyridine (82). The mixture of 81 (1.2 g, 5 mmol), cyclopropylboronic acid ( $859 \mathrm{mg}, 10 \mathrm{mmol}), \mathrm{Cu}(\mathrm{OAc})_{2}(900 \mathrm{mg}, 5 \mathrm{mmol}), 2,2^{\prime}$-Bipyridine $(780 \mathrm{mg}$, $5 \mathrm{mmol})$ and $\mathrm{Na}_{2} \mathrm{CO}_{3}(1.0 \mathrm{~g}, 10 \mathrm{mmol})$ in $30 \mathrm{~mL}$ 1,2-DCE was stirred at $70^{\circ} \mathrm{C}$ for $2 \mathrm{~h}$. After filtration, the filtrate was concentrated under vacuum. The residue was dissolved in EA and washed with water for 4 times and dried using anhydrous sodium sulfate. EA was evaporated under vacuum. The residue was purified by column chromatography on silico gel (eluted with PE/EA, 2/1) to afford $714 \mathrm{mg}$ product as yellow solid. Yield: 51\%.<smiles>O=S(=O)(c1cc2cnn(C3CC3)c2cn1)N1CCC(O)CC1</smiles>

1-((1-cyclopropyl-1H-pyrazolo[3,4-c]pyridin-5-yl)sulfonyl)piperidin-4-ol (83a). 
<smiles>O=S(=O)(c1cc2cnn(C3CC3)c2cn1)N1CCOCC1</smiles>

4-((1-cyclopropyl-1H-pyrazolo[3,4-c]pyridin-5-yl)sulfonyl)morpholine ( $\mathbf{8 3 \boldsymbol { b }})$.

To the solution of $82(140 \mathrm{mg}, 0.5 \mathrm{mmol})$ in $5 \mathrm{~mL} \mathrm{CH}_{3} \mathrm{CN}, 100 \mu \mathrm{L} \mathrm{H}_{2} \mathrm{O}$ and $100 \mu \mathrm{L} \mathrm{AcOH}$ was added 5,5-dimethylhydantoin $(200 \mathrm{mg}, 1.0 \mathrm{mmol})$ slowly at $-15{ }^{\circ} \mathrm{C}$ under stirring. The mixture was kept at $-15{ }^{\circ} \mathrm{C}$ for $1 \mathrm{~h}$. EA was added; the organic phase was washed with brine and dried by anhydrous sodium sulfate. EA was evaporated under vacuum.

To the solution of the above product in DCM was added 4-hydroxylpiperidine (100 $\mathrm{mg}, 1 \mathrm{mmol}$, for 83a) or morpholine ( $87 \mathrm{mg}, 1 \mathrm{mmol}$, for 83b) and TEA ( $277 \mu \mathrm{L}, 1 \mathrm{mmol})$. The mixture was stirred at room temperature for $30 \mathrm{~min}$. Then it was washed with water and brine, and dried using anhydrous sodium sulfate. DCM was evaporated under vacuum to afford the product, which can be used for next step without further purification.<smiles>CC(=O)OC1CCN(S(=O)(=O)c2cc3c(Br)nn(C4CC4)c3cn2)CC1</smiles>

1-((3-bromo-1-cyclopropyl-1H-pyrazolo[3,4-c]pyridin-5-yl)sulfonyl)piperidin-4-yl acetate (84a). To the solution of 83a (crude product, $4 \mathrm{mmol})$ in EA was added $\mathrm{Ac}_{2} \mathrm{O}(2.0 \mathrm{~mL}, 20 \mathrm{mmol})$ and pyridine $(1480 \mu \mathrm{L}, 16 \mathrm{mmol})$. The mixture was stirred at reflux until disappearance of the substrate. The organic phase was washed with water and brine and dried using anhydrous sodium sulfate. EA was evaporated under vacuum. The crude product was dissolved in TFA/AcOH $(8 / 8 \mathrm{~mL})$. To the solution was added NBS $(1.42 \mathrm{~g}, 8 \mathrm{mmol})$. The mixture was stirred overnight at $50{ }^{\circ} \mathrm{C}$. The solvent was evaporated under vacuum. The residue was dissolved in ethyl acetate and washed with saturated aqueous $\mathrm{NaHCO}_{3}$ and brine. After evaporation, the residue was purified by column chromatography on silico gel (eluted with PE/EA, 2/1) to afford $900 \mathrm{mg} 74 \mathrm{a}$ as white powder. Yield: 51\% for two steps. ${ }^{1} \mathrm{H}$ NMR $(400 \mathrm{MHz}$, Chloroform- $d$ ) $\delta 9.13(\mathrm{~d}, J=1.2 \mathrm{~Hz}, 1 \mathrm{H}), 8.24(\mathrm{~d}, J=1.2 \mathrm{~Hz}, 1 \mathrm{H}), 4.82$ (tt, $J=7.9,3.8 \mathrm{~Hz}, 1 \mathrm{H}), 3.86-3.71(\mathrm{~m}, 1 \mathrm{H}), 3.61(\mathrm{ddd}, J=11.7,7.0,4.0 \mathrm{~Hz}, 2 \mathrm{H}), 3.21$ (ddd, $J=$ $12.4,8.5,3.6 \mathrm{~Hz}, 2 \mathrm{H}), 2.01$ (s, 3H), 1.95 (ddd, $J=13.9,7.2,3.6 \mathrm{~Hz}, 2 \mathrm{H}), 1.76$ (dtd, $J=12.5,8.3$, $3.8 \mathrm{~Hz}, 2 \mathrm{H}), 1.32(\mathrm{tq}, J=6.9,2.6,2.0 \mathrm{~Hz}, 4 \mathrm{H})$.<smiles>O=S(=O)(c1cc2c(Br)nn(C3CC3)c2cn1)N1CCOCC1</smiles>

4-((3-bromo-1-cyclopropyl-1H-pyrazolo[3,4-c]pyridin-5-yl)sulfonyl)morpholine (84b). To the solution of $83 \mathbf{b}(97 \mathrm{mg}, 0.3 \mathrm{mmol})$ in $\mathrm{CH}_{3} \mathrm{CN} / \mathrm{AcOH}(3 / 3 \mathrm{~mL})$ was added dibromo-5,5dimethylhydantoin (129 mg, $0.45 \mathrm{mmol})$. The mixture was stirred at room temperature. Upon the disappearance of $\mathbf{8 3 b}$, EA was added. The organic phase was washed with saturated aqueous $\mathrm{NaHCO}_{3}$ and brine. After evaporation, the residue was purified by column chromatography on silico 
gel to afford $68 \mathrm{mg} 84 \mathrm{~b}$ as white powder. Yield: $59 \%$.<smiles></smiles>

1-((1-cyclopropyl-3-iodo-1H-indazol-5-yl)sulfonyl)piperidin-4-yl acetate (85). To the solution of the substrate (crude product, $1 \mathrm{mmol}$ ) in $\mathrm{CH}_{3} \mathrm{CN} / \mathrm{H}_{2} \mathrm{SO}_{4}(8 / 2 \mathrm{~mL}$ ) was added NIS $(562 \mathrm{mg}, 2.5$ mmol). The mixture was stirred at room temperature for $48 \mathrm{~h}$. EA was added. The organic phase was washed with sat. aq. $\mathrm{NaHCO}_{3}$ and brine and dried with $\mathrm{Na}_{2} \mathrm{SO}_{4}$. After evaporating the EA, the residue was purified with column chromatography on silico gel (eluted with PE/EA, 2/1) to afford $180 \mathrm{mg}$ product as white powder. Yield: $37 \%$

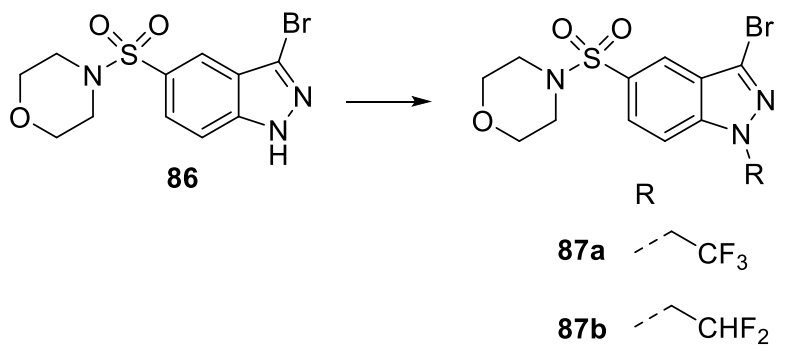

To the solution of 86 (70 mg, $0.2 \mathrm{mmol}$ ) in $2 \mathrm{~mL} \mathrm{DMF}$ was added 2-iodo-1,1,1-trifluoroethane (for 87a, $84 \mathrm{mg}, 0.4 \mathrm{mmol}$ ) or 2-iodo-1,1-difluoroethane (for $87 \mathrm{~b}, 76 \mathrm{mg}, 0.4 \mathrm{mmol}$ ) and $\mathrm{K}_{2} \mathrm{CO}_{3}(56 \mathrm{mg}$, $0.4 \mathrm{mmol}$ ). The mixture was stirred at $80^{\circ} \mathrm{C}$ overnight. After filtration, the filtrate was concentrated under vacuum and the residue was purified with column chromatography on silico gel (eluted with PE/EA, 3/2) to afford the product 87a (39 mg, yield 45\%), 87b (47 mg, yield 58\%).

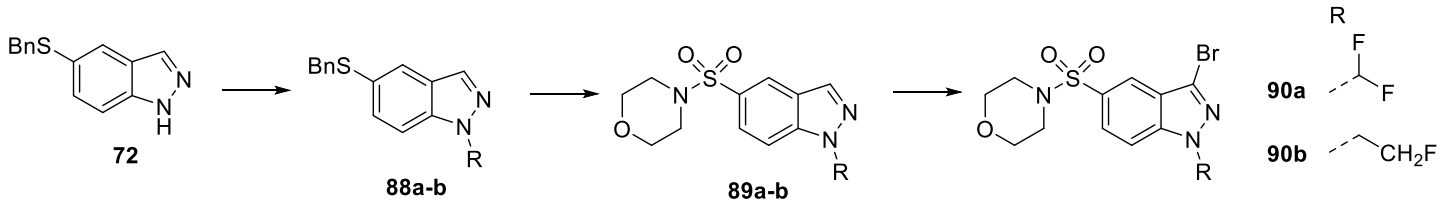

5-(benzylthio)-1-(difluoromethyl)-1H-indazole (88a). To the solution of $72(240 \mathrm{mg}, 1 \mathrm{mmol})$ in $\mathrm{CH}_{3} \mathrm{CN}$ was added Diethyl bromodifluoromethanephosphonate (534 mg, $2 \mathrm{mmol}$ ) and $\mathrm{KF}$ (116 mg, $2 \mathrm{mmol}$ ). The mixture was stirred at $40{ }^{\circ} \mathrm{C}$ for $48 \mathrm{~h}$. After filtration, the filtrate was concentrated under vacuum and the residue was purified with column chromatography on silico gel (eluted with PE/EA, 12/1) to afford the product 88a (245 mg, yield 84\%). ${ }^{1} \mathrm{H}$ NMR (400 MHz, Chloroform- $d$ ) $\delta$ $8.24(\mathrm{~s}, 1 \mathrm{H}), 7.65(\mathrm{~d}, J=9.1 \mathrm{~Hz}, 1 \mathrm{H}), 7.61-7.23(\mathrm{~m}, 8 \mathrm{H}), 4.16(\mathrm{~s}, 2 \mathrm{H})$.

5-(benzylthio)-1-(2-fluoroethyl)-1H-indazole ( $88 \boldsymbol{b})$. To the solution of $72(240 \mathrm{mg}, 1 \mathrm{mmol})$ in DMF was added 1-Fluoro-2-iodoethane ( $348 \mathrm{mg}, 2 \mathrm{mmol}$ ) and $\mathrm{K}_{2} \mathrm{CO}_{3}(276 \mathrm{mg}, 2 \mathrm{mmol})$. The mixture was stirred at $80^{\circ} \mathrm{C}$ overnight. After filtration, the filtrate was concentrated under vacuum and the residue was purified with column chromatography on silico gel (eluted with PE/EA, 2/1) to afford the product $\mathbf{8 8 b}, 186 \mathrm{mg}$, yield $65 \%$.

The procedure for synthesis of $\mathbf{8 9} \mathbf{a}-\mathbf{b}$ was as the described in the synthesis of $\mathbf{8 3 \mathbf { b }}$. 4-((3-bromo-1-(difluoromethyl)-1H-indazol-5-yl)sulfonyl)morpholine (90a). To the solution of 89a (98 $\mathrm{mg}, 0.31 \mathrm{mmol}$ ) in $4 \mathrm{~mL} \mathrm{CH} \mathrm{CHN}_{3}$ and $2 \mathrm{~mL}$ TFA was added NBS ( $83 \mathrm{mg}, 0.465 \mathrm{mmol}$ ). The mixture was stirred at $80{ }^{\circ} \mathrm{C}$. Upon the disappearance of 89a, ethyl acetate was added. The organic phase was washed with saturated aqueous $\mathrm{NaHCO}_{3}$ and brine. After evaporation, the residue was purified by column chromatography on silico gel to afford $61 \mathrm{mg}$ 90a. Yield: 50\%. 
4-((3-bromo-1-(2-fluoroethyl)-1H-indazol-5-yl)sulfonyl)morpholine (90b). To the solution of 89b ( $0.30 \mathrm{mmol}$, crude product) in $4 \mathrm{~mL} \mathrm{CH} 3 \mathrm{CN}$ and $2 \mathrm{~mL} \mathrm{AcOH}$ was added NBS (169 mg, $0.95 \mathrm{mmol}$ ). The mixture was stirred at $40{ }^{\circ} \mathrm{C}$ overnight. Ethyl acetate was added. The organic phase was washed with saturated aqueous $\mathrm{NaHCO}_{3}$ and brine. After evaporation, the residue was purified by column chromatography on silico gel to afford $87 \mathrm{mg} \mathbf{9 0 b}$. Yield: $74 \%$. 
${ }^{1} \mathrm{H}$ NMR and ${ }^{13} \mathrm{C}$ NMR spectrum

\section{Compound 1}

20190306 wct-1095-1-H/10
PROTON DUSO [D: LNIR_DATA\} LG 16 LIVIDDATA\} LG 16

'H NMR (400 MHz, DMSO- $\left.d_{6}\right) \delta 12.63(\mathrm{~s}, 1 \mathrm{H}), 9.53(\mathrm{~s}, 1 \mathrm{H}), 8.56(\mathrm{~s}, 1 \mathrm{H}), 8.11(\mathrm{~s}, 1 \mathrm{H}), 7.92-7.45(\mathrm{~m}$, $3 \mathrm{H}), 7.35(\mathrm{t}, J=9.3 \mathrm{~Hz}, 1 \mathrm{H}), 4.65(\mathrm{~s}, 1 \mathrm{H}), 3.49(\mathrm{~s}, 1 \mathrm{H}), 3.16(\mathrm{~s}, 2 \mathrm{H}), 2.71(\mathrm{~s}, 2 \mathrm{H}), 1.74(\mathrm{~s}, 2 \mathrm{H}), 1.44(\mathrm{~s}$,

$2 \mathrm{H})$<smiles>CC=CCCC</smiles><smiles>C1CCCCCC1</smiles><smiles>CCCCCCC</smiles>

HO<smiles>CNc1n[nH]c2ccc(S(=O)(=O)N3CCC(C)CC3)cc12</smiles><smiles>Fc1ccc(Cl)c(Cl)c1</smiles>

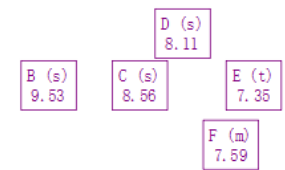

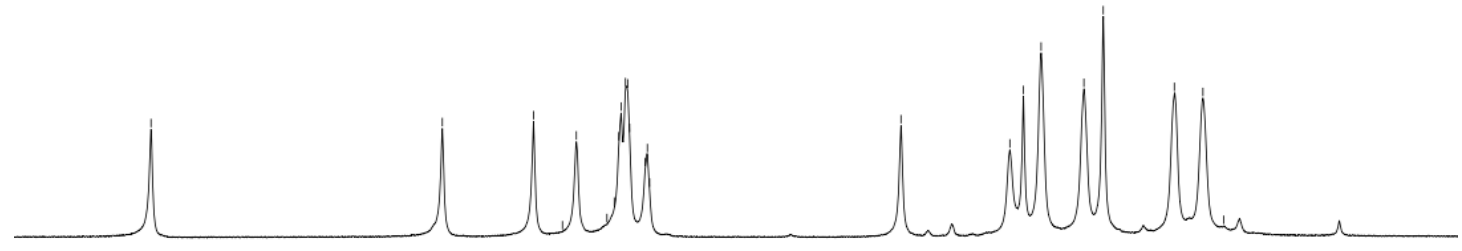

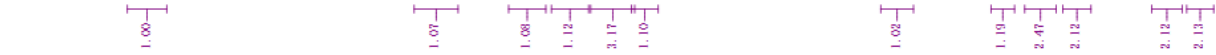

$\begin{array}{lllllllllllllllllllllllllllllllllllllll}4.0 & 13.5 & 13.0 & 12.5 & 12.0 & 11.5 & 11.0 & 10.5 & 10.0 & 9.5 & 9.0 & 8.5 & 8.0 & 7.5 & 7.0 & 6.5 & 6.0 & 5.5 & 5.0 & 4.5 & 4.0 & 3.5 & 3.0 & 2.5 & 2.0 & 1.5 & 1.0 & 0.5 & 0.0 & -0.5 & -1.0\end{array}$ $20190306-$ wct-1095-1-C/10

C13CPD DUSO [D: \NIXR_DATA\} LQ

$\frac{1}{1} \frac{1}{1}$

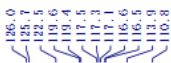

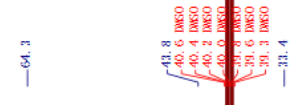

${ }_{13} \mathrm{CNMR}\left(101 \mathrm{MHz}\right.$, DMSO-d $\left.d_{6}\right) \delta 151.27(\mathrm{~d}, J=237.9 \mathrm{~Hz}), 146.25,141.74,140.03,126.01,125.69$

$122.46,119.53(\mathrm{~d}, J=18.1 \mathrm{~Hz}), 117.43(\mathrm{~d}, J=21.6 \mathrm{~Hz}), 117.08,116.53(\mathrm{~d}, J=6.1 \mathrm{~Hz}), 113.89,110.83$, $64.29,43.75,33.38$.
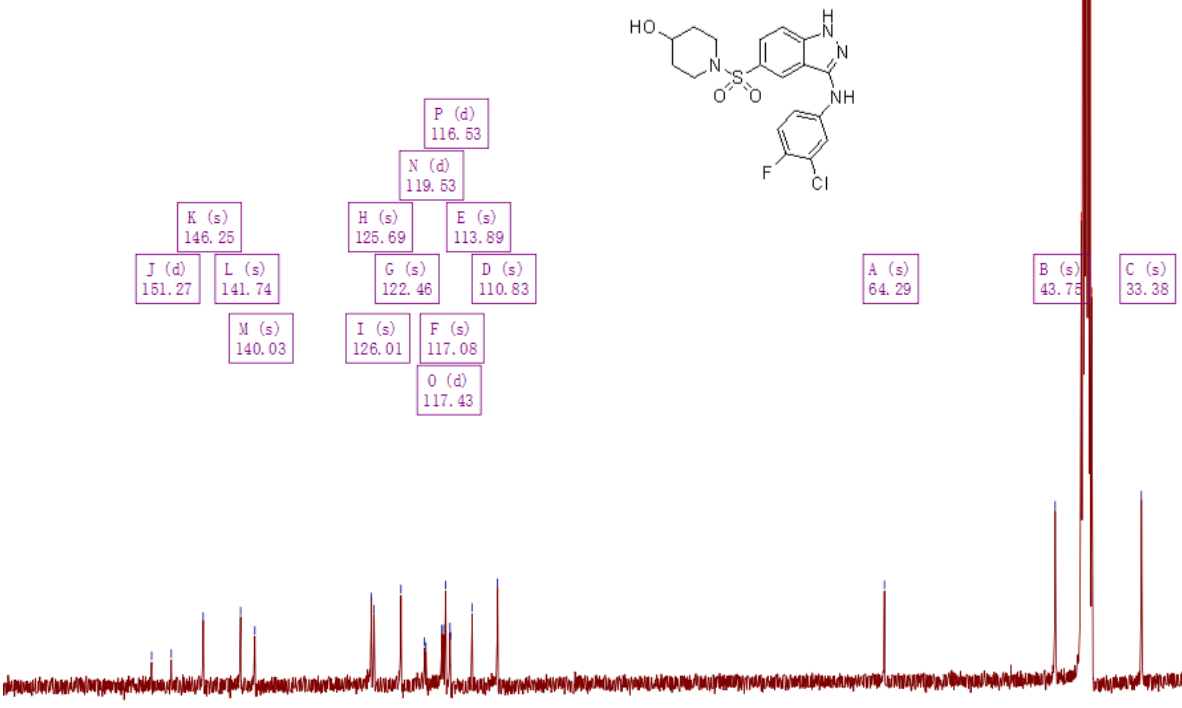

$70 \quad 160 \quad 150 \quad 140 \quad 130 \quad 120 \quad 110 \quad 100 \quad 90,80$ 


\section{Compound 2.}

20190705-wct-1117-2-H/10
PROTON DUSO \{D: \NIR_DATA\} LG 33

H NMR ( $\left.400 \mathrm{MHz}, \mathrm{DMSO}-d_{\mathrm{k}}\right) 89.55(\mathrm{~s}, 1 \mathrm{H}), 8.55(\mathrm{~s}, 1 \mathrm{H}), 8.03$ (dd, $\left.J=6.6,2.7 \mathrm{~Hz}, 1 \mathrm{H}\right), 7.76-7.64$

$(\mathrm{m}, 2 \mathrm{H}), 7.59$ (ddd, $J=9.1,4.1,2.7 \mathrm{~Hz}, 1 \mathrm{H}), 7.36(\mathrm{t}, J=9.1 \mathrm{~Hz}, 1 \mathrm{H}), 4.64(\mathrm{~d}, J=3.8 \mathrm{~Hz}, 1 \mathrm{H}), 3.98(\mathrm{~s}$,

$3 \mathrm{H}), 3.50(\mathrm{tq}, J=7.6,3 . \mathrm{Hz}, \mathrm{H}), 3.16(\mathrm{ddt}, J=10.7,6.7,3.5 \mathrm{~Hz}, 2 \mathrm{H}), 2.71$ (ddd, $J=11.9,8.5,3.5 \mathrm{~Hz}$

$2 \mathrm{H}), 1.81-1.68(\mathrm{~m}, 2 \mathrm{H}), 1.44$ (dtd, $J=12.2,7.9,3.5 \mathrm{~Hz}, 2 \mathrm{H}$ ).
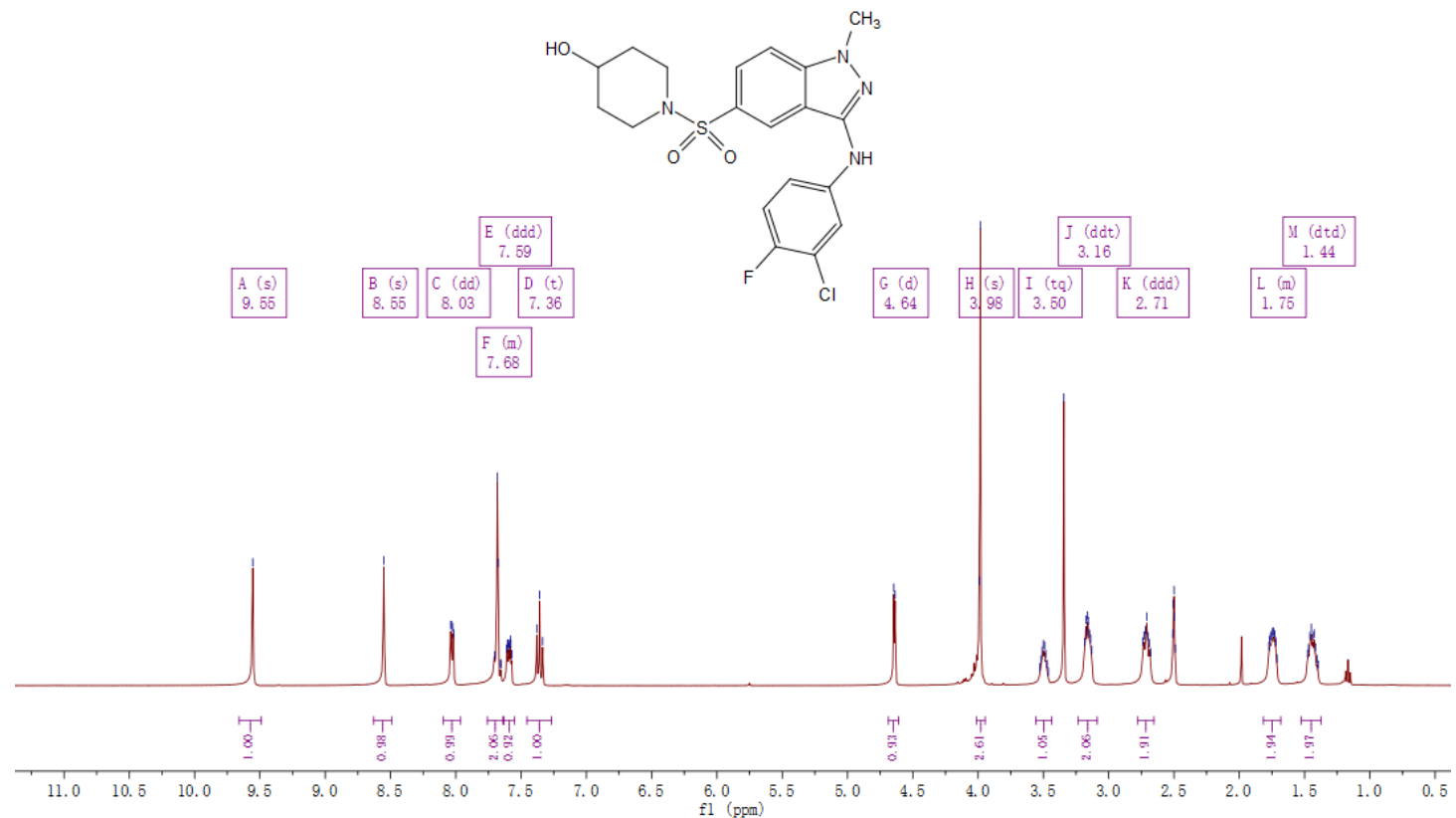<smiles>C/C=C/Nc1nn(C)c2ccc(S(=O)(=O)N3CCC(O)CC3)cc12</smiles>

$20190705-w c t-1117-2-\mathrm{C} / 10$

C13CPD DUSO \{D: $\backslash$ NNR

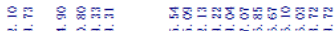

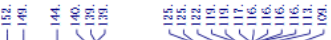

${ }^{13} \mathrm{C} \mathrm{NMR}\left(101 \mathrm{MHz}\right.$, DMSO- $\left.d_{l}\right) \delta 150.91$ (d, $\left.J=238.4 \mathrm{~Hz}\right), 144.90,140.80,139.32$ (d, $\left.J=2.3 \mathrm{~Hz}\right)$,

$125.54,125.09,122.13,119.13(\mathrm{~d}, J=18.1 \mathrm{~Hz}), 116.96(\mathrm{~d}, J=21.7 \mathrm{~Hz}), 116.67,116.06(\mathrm{~d}, J=6.5 \mathrm{~Hz})$,

$113.72,109.72,63.81,43.27,35.37,32.90$

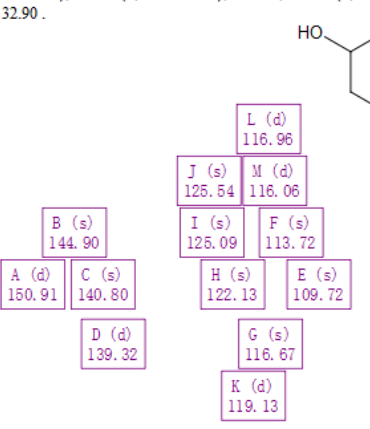
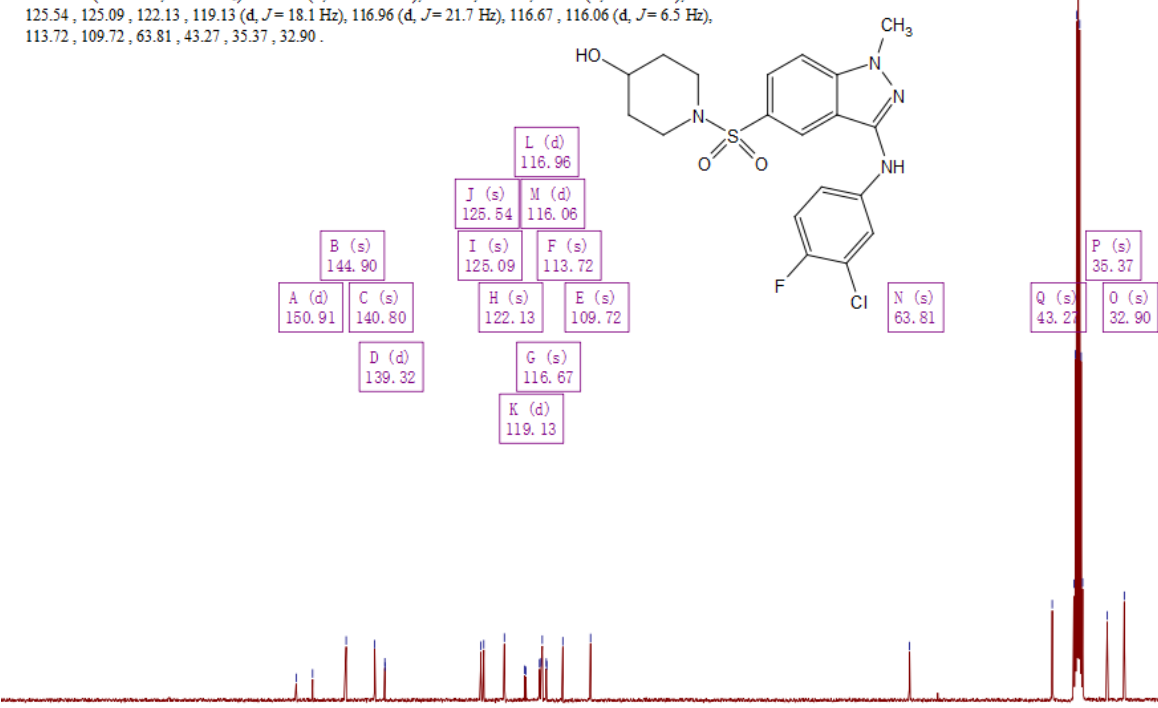

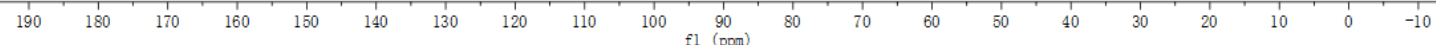




\section{Compound 3.}
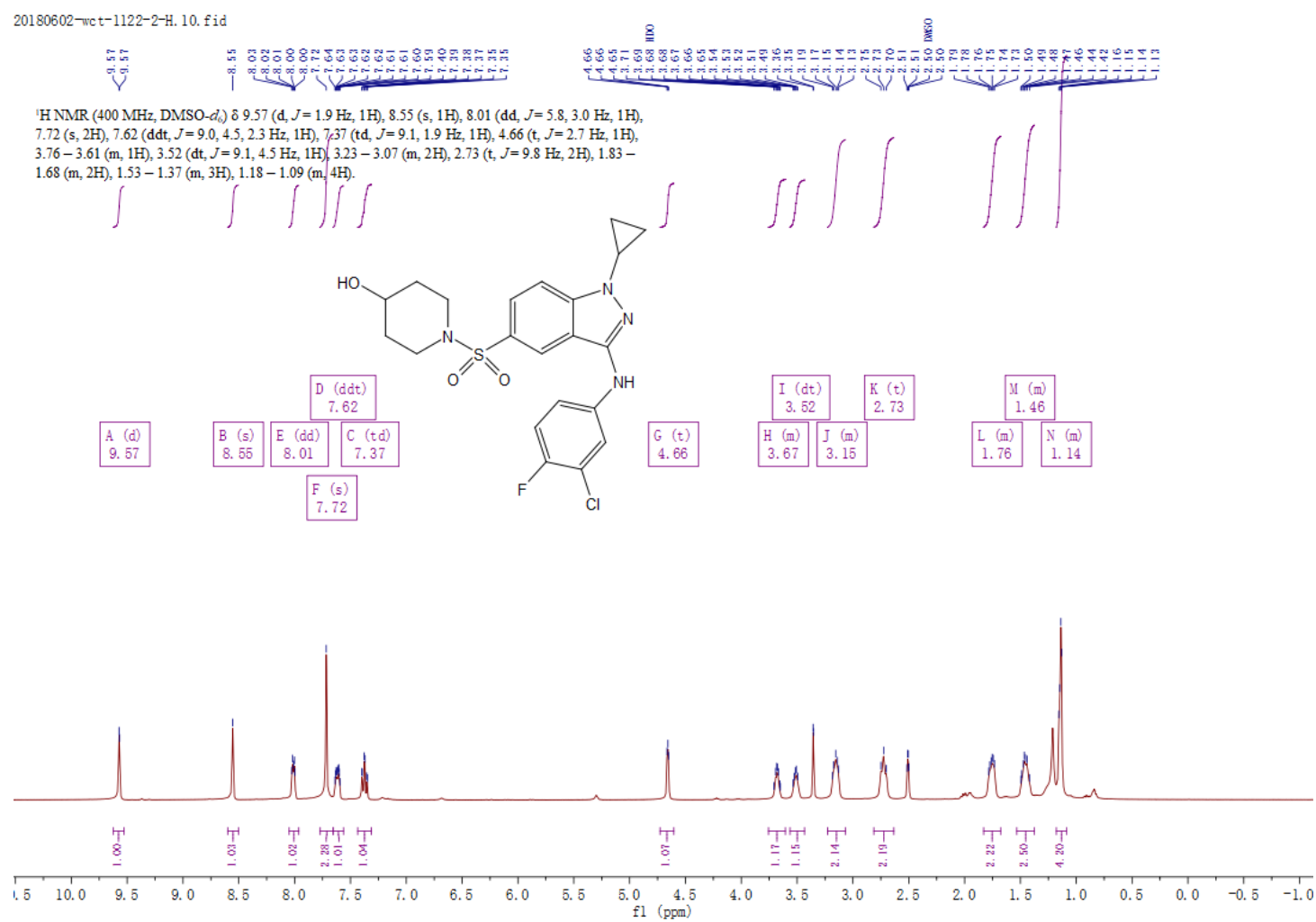

20190623-wct-1122-2-C/10

C13CPD DUSO [D: \{NIR_DATA\} LG 54.

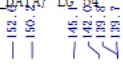

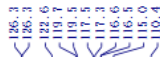

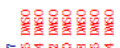

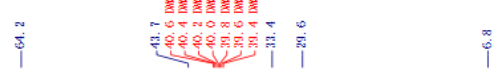

${ }^{13} \mathrm{C} \mathrm{NMR}\left(101 \mathrm{MHz}, \mathrm{DMSO}-d_{\mathrm{d}}\right) \delta 151.4(\mathrm{~d}, J=238.5 \mathrm{~Hz}), 145.1,142.0,139.7(\mathrm{~d}, J=2.5 \mathrm{~Hz}), 126.3$,

$126.3,122.6,119.6(\mathrm{~d}, J=18.2 \mathrm{~Hz}), 117.5,117.3,116.6(\mathrm{~d}, J=6.5 \mathrm{~Hz}), 115.0,110.4,64.2,43.7,33$.

$, 29.6,6.8$

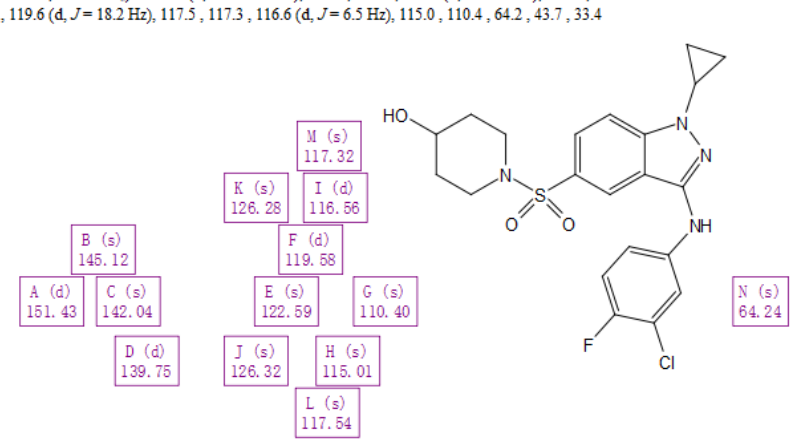

\begin{tabular}{cc}
\hline$(s)$ \\
64.24 \\
\hline
\end{tabular}

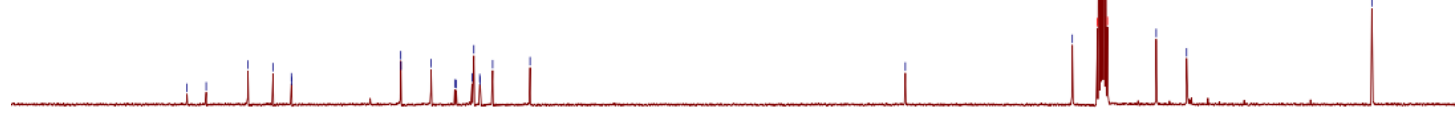




\section{Compound 4.}

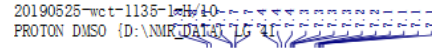

洔

I $\mathrm{HNMR}\left(400 \mathrm{MHz}, \mathrm{DMSO}-\mathrm{d}_{\mathrm{l}}\right) \delta 7.81(\mathrm{~d}, J=8.9 \mathrm{~Hz}, 1 \mathrm{H}), 7.66$ (dd, $\left.J=8.9,1.6 \mathrm{~Hz}, 1 \mathrm{H}\right), 7.38(\mathrm{t}, J=$

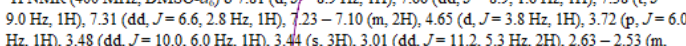
$2 \mathrm{H}), 1.79-1.66(\mathrm{~m}, 2 \mathrm{H}), 1.42$ (dtd, $J \neq 11.9,8.0,3.5 \mathrm{~Hz}, 2 \mathrm{H}), 1.16(\mathrm{~d}, J=3.3 \mathrm{~Hz}, 4 \mathrm{H}$ ).
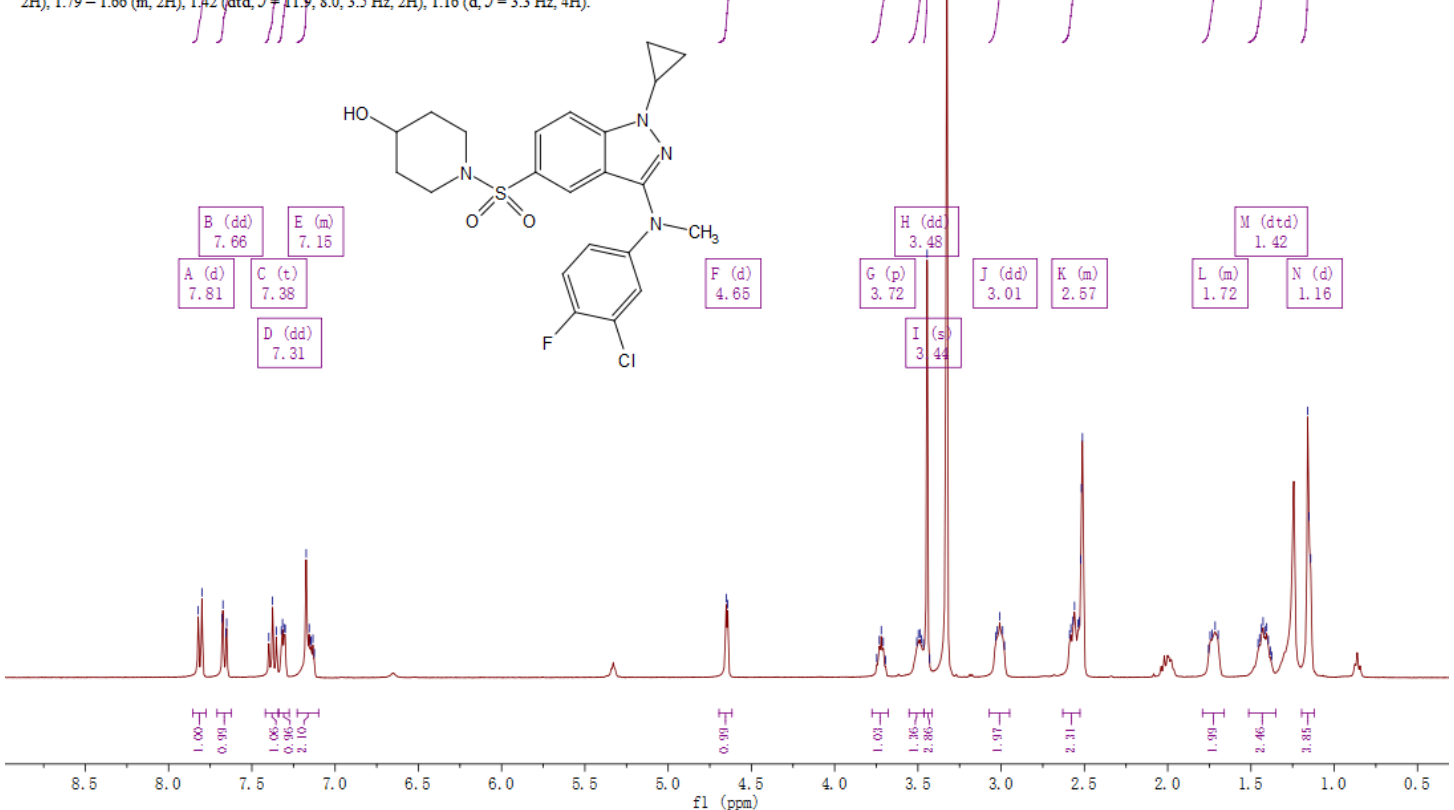

$\underbrace{2}$

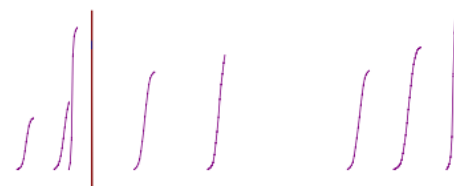

$20190528-w c t-1135-1-\mathrm{C} / 10$

C13CPD DUSO \{D: \NIR, DATA 1
I

${ }^{13} \mathrm{C} N M R\left(101 \mathrm{MHz}\right.$, DMSO- $\left.d_{\mathrm{l}}\right) \delta 153.48(\mathrm{~d}, J=242.0 \mathrm{~Hz}), 148.50,144.79(\mathrm{~d}, J=2.9 \mathrm{~Hz}), 143.38$, $130.11,126.57,125.41,122.44(\mathrm{~d}, J=11.8 \mathrm{~Hz}), 121.49(\mathrm{~d}, J=6.8 \mathrm{~Hz}), 120.28(\mathrm{~d}, J=18.5 \mathrm{~Hz}), 117.74$ $(\mathrm{d}, J=22.0 \mathrm{~Hz}), 115.15,111.46,64.23,43.66,40.80,33.34,29.77,6.80$
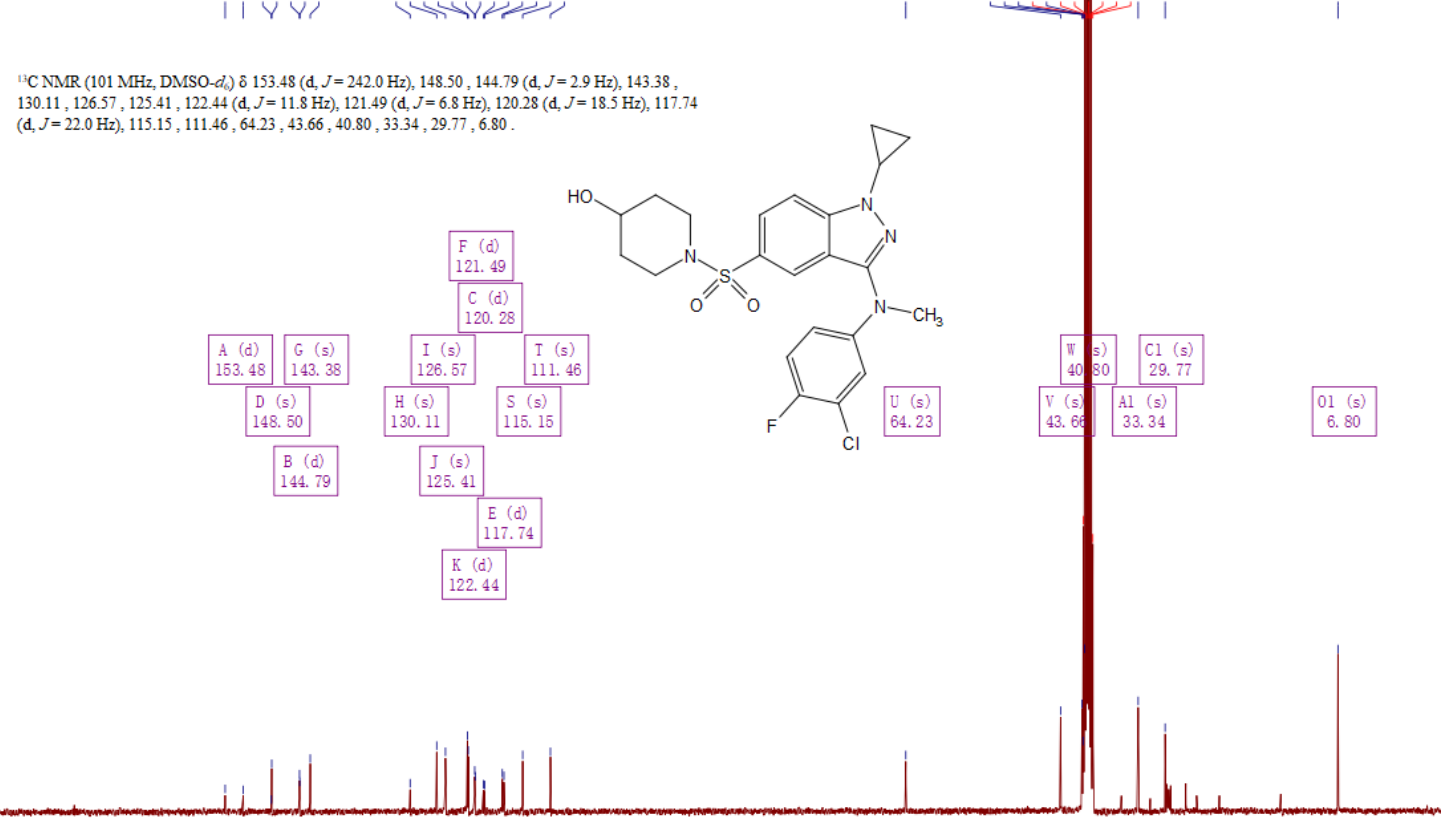

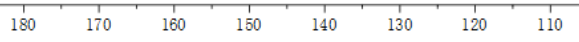

$100 \quad 90$




\section{Compound 5.}

20190503-wct-1370-3-H/10
PROTON DUSO \{D: $\backslash$ NMR_DATA\} LG 1

H NMR ( $400 \mathrm{MHz}$, DMSO-d $) \delta 7.88$ (d, $J=8.9 \mathrm{~Hz}, 1 \mathrm{H}), 7.83$ (d, $J=1.5 \mathrm{~Hz}, 1 \mathrm{H}), 7.77$ (dd, $J=8.9$,

$1.7 \mathrm{~Hz}, 1 \mathrm{H}), 7.64(\mathrm{dd}, J=6.2,3.0 \mathrm{~Hz}, 1 \mathrm{H}), 7.49(\mathrm{t}, J=9.0 \mathrm{~Hz}, 1 \mathrm{H}), 7.36(\mathrm{dt}, J=9.1,3.5 \mathrm{~Hz}, 1 \mathrm{H}), 4.65(\mathrm{~s}$,

$1 \mathrm{H}), 3.72(\mathrm{tt}, J=6.8,4.1 \mathrm{~Hz}, 1 \mathrm{H}), 3.49(\mathrm{dq}, J=7.7,4.0 \mathrm{~Hz}, 1 \mathrm{H}), 3.11(\mathrm{ddd}, J=1.2,7.1,3.7 \mathrm{~Hz}, 2 \mathrm{H})$,

2.69 (ddd, $J=11.7,8.3,3.4 \mathrm{~Hz}, 2 \mathrm{H}), 1.73$ (ddt, $J=13.9,7.2,3.5 \mathrm{~Hz}, 2 \mathrm{H}), 1.42$ (dtd, $J=12.2,8.1,3.7$
$\mathrm{~Hz}, 2 \mathrm{H}), 1.11$ (dq, $J=7.7,2.5 \mathrm{~Hz}, 4 \mathrm{H}$ ).<smiles>O=S(=O)(c1ccc2c(c1)c(Oc1ccc(F)c(Cl)c1)nn2C1CC1)N1CCC(O)CC1</smiles>

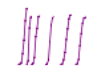<smiles>C=CC1CCCCC1CC</smiles>
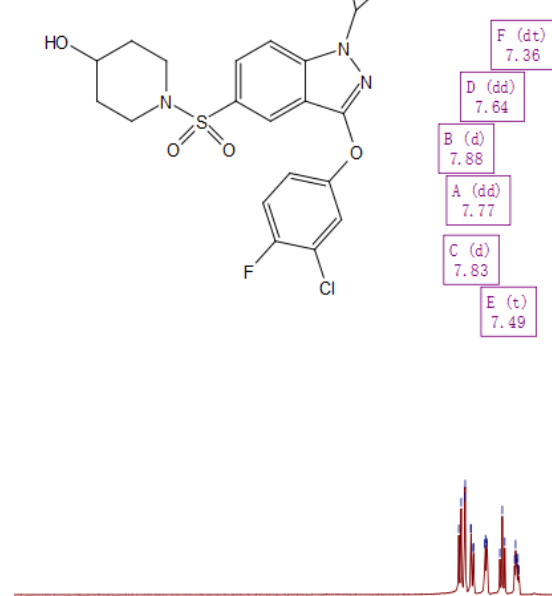

U. N

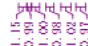

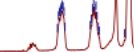

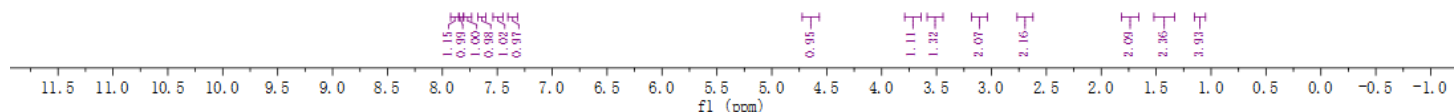

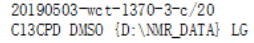

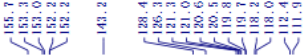

${ }^{13} \mathrm{CNMR}\left(101 \mathrm{MHz}, \mathrm{DMSO}-d_{\mathrm{c}}\right) 8154.54$ (d, $\left.J=243.2 \mathrm{~Hz}\right), 153.04,152.17$ (d, $J=2.9 \mathrm{~Hz}$ ), 143.23 , $128.42,126.33,121.27,120.97,120.55(\mathrm{~d}, J=19.6 \mathrm{~Hz}), 119.72(\mathrm{~d}, J=7.3 \mathrm{~Hz}), 118.10(\mathrm{~d}, J=23.1 \mathrm{~Hz})$ $112.40,111.87,64.19,43.64,33.35,29.97,6.91$
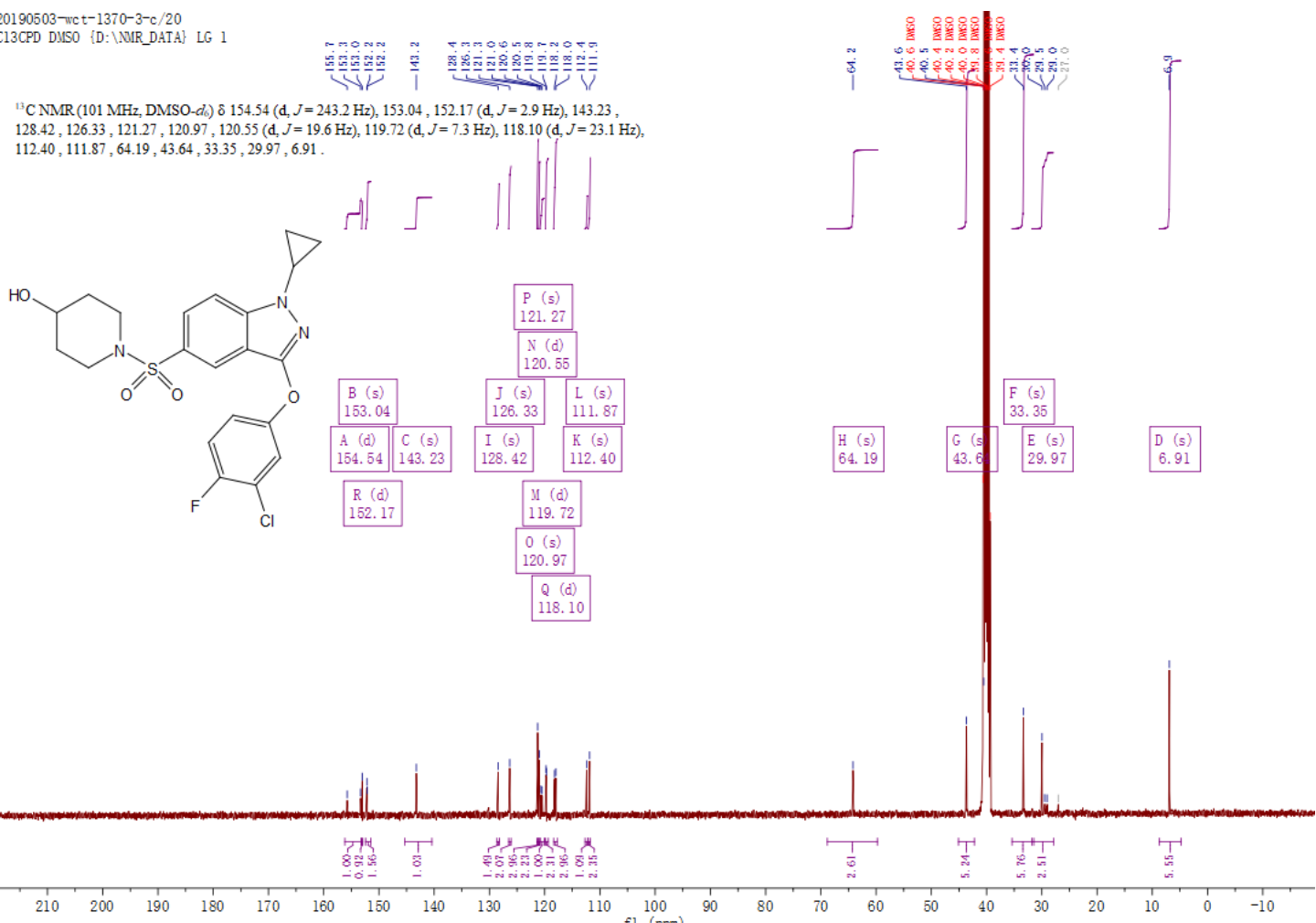


\section{Compound 6.}

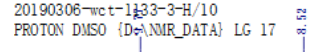

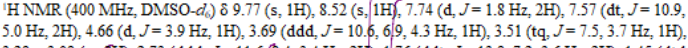
$3.29-3.09(\mathrm{~m}, 2 \mathrm{H}), 2.73$ (ddd, $J=11.6,8.4,3.4 \mathrm{~Hz}, 2 \mathrm{H}), 1.76$ (ddt, $J=13.9,7.2,3.6 \mathrm{~Hz}, 2 \mathrm{H}), 1.45$ (ddd, $J=12.0,8.0,3.6 \mathrm{~Hz}, 2 \mathrm{H}), 1.24-1.09(\mathrm{~m}, 4 \mathrm{H})$

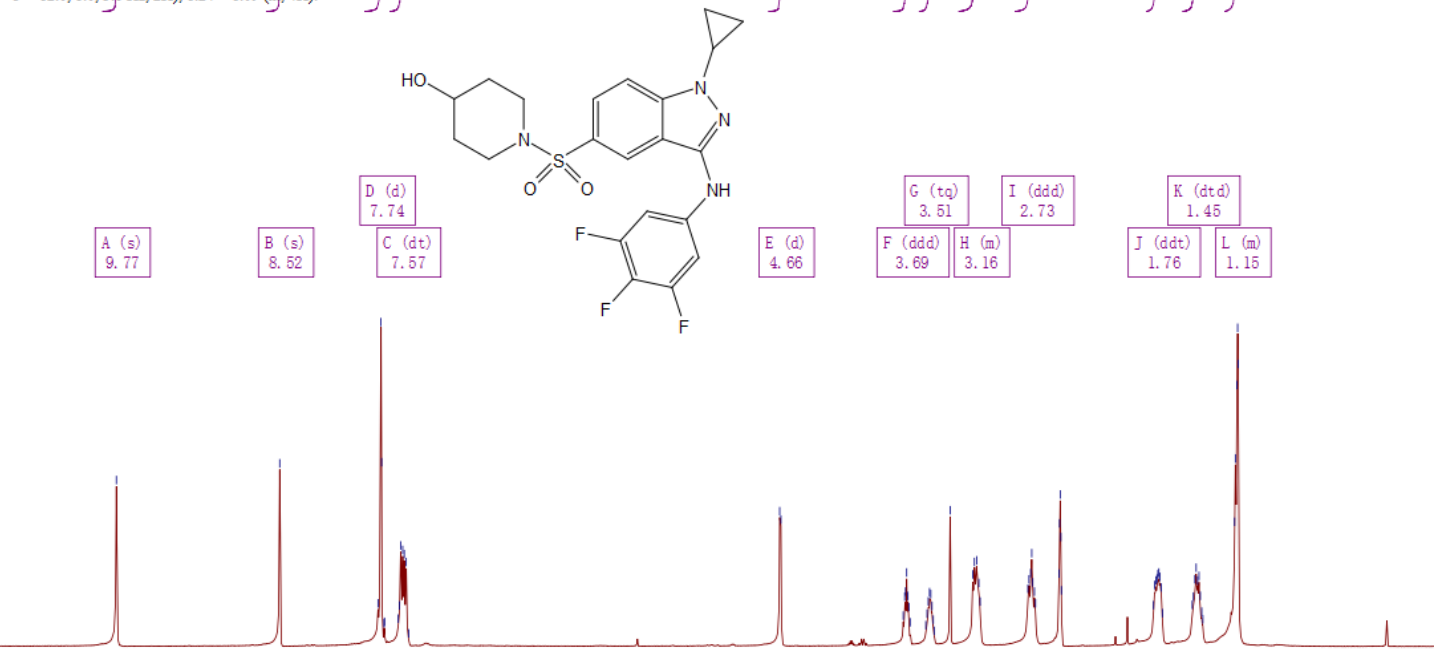

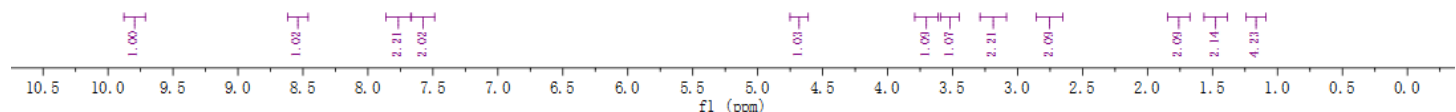

$20190306-$ wct $-1133-3-\mathrm{C} / 10$

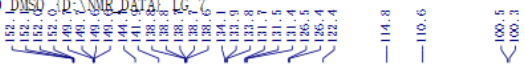

${ }^{13} \mathrm{CNMR}\left(101 \mathrm{MHz}, \mathrm{DMSO}-d_{d}\right) \delta 150.84$ (ddd, $\left.J=242.6,9.9,6.1 \mathrm{~Hz}\right), 144.71,141.92,139.39$ -

$138.31(\mathrm{~m}), 132.73$ (dt, $J=239.8,16.1 \mathrm{~Hz}), 126.51,126.38,122.40,114.83,110.57,100.41$ (d, $J=24.7$

$\mathrm{Hz}), 64.20,43.69,33.35,29.67,6.85$
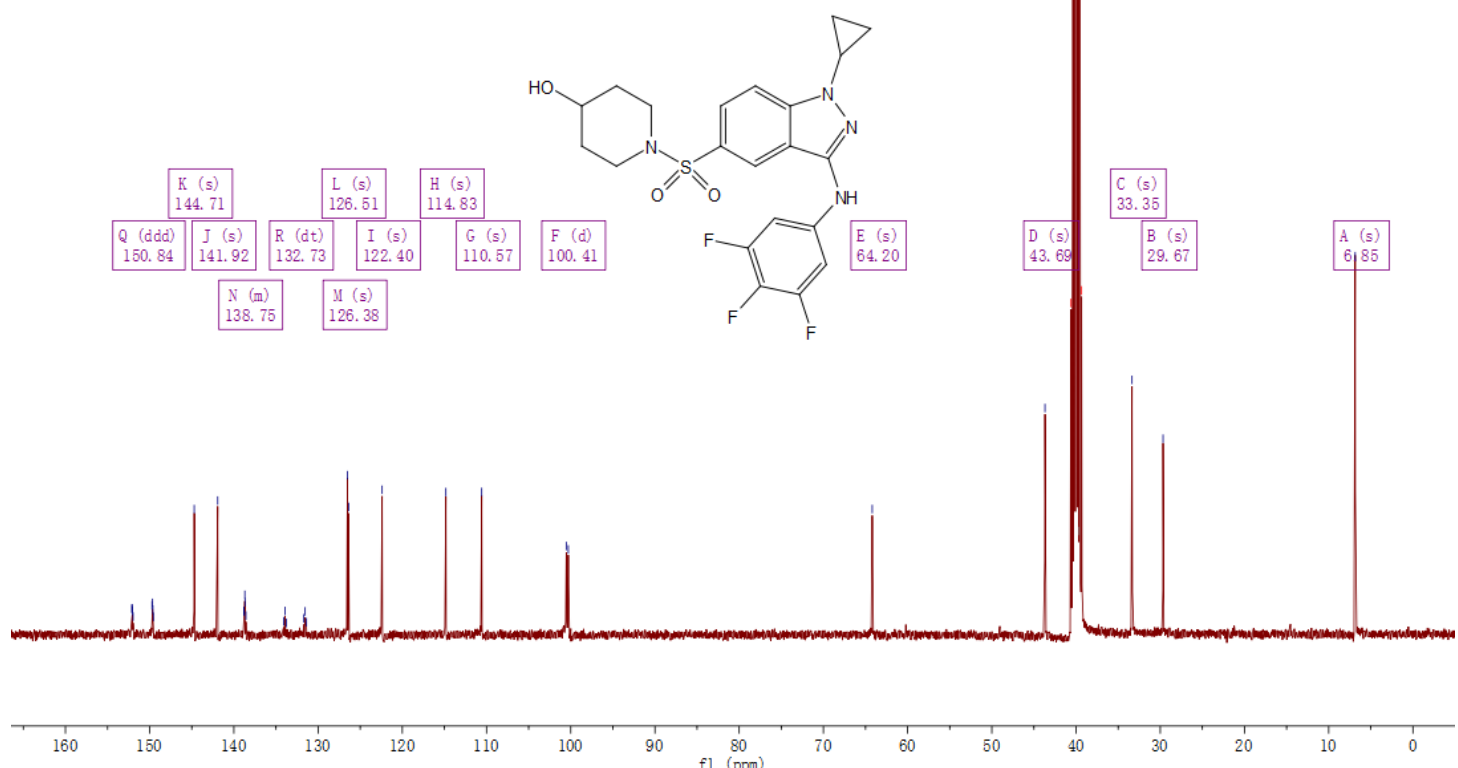

80
$(\mathrm{ppm})$

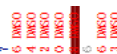

过年果得

$\begin{array}{lllllllll}160 & 150 & 140 & 130 & 120 & 110 & 100 & 90 & 80\end{array}$ 


\section{Compound 7}

$20190306-$ wet $-1165-1-H / 10$
PROTON DUSO \{D: $\backslash$ NIR_DATA $\}$ LG 8

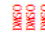

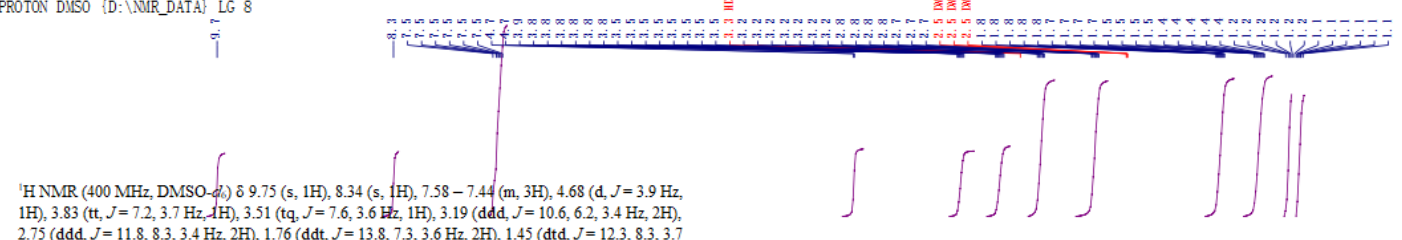

$1 \mathrm{H}), 3.83(\mathrm{tt}, J=7.2,3.7 \mathrm{~Hz}, 1 \mathrm{H}), 3.51$ (tq, $J=7.6,3.6 \mathrm{~Hz}, 1 \mathrm{H}), 3.19$ (ded, $J=10.6,6.2,3.4 \mathrm{~Hz}, 2 \mathrm{H}$ ),

$2.75(\mathrm{ddd}, J=11.8,8.3,3.4 \mathrm{~Hz}, 2 \mathrm{H}), 1.76(\mathrm{ddt}, J=13.8,7.3,3.6 \mathrm{~Hz}, 2 \mathrm{H}), 1.45$ (dtd, $J=12.3,8.3,3$. $\mathrm{Hz}, 2 \mathrm{H}), 1.25-1.19(\mathrm{~m}, 2 \mathrm{H}), 1.15-1.09(\mathrm{~m}, 2 \mathrm{H})$
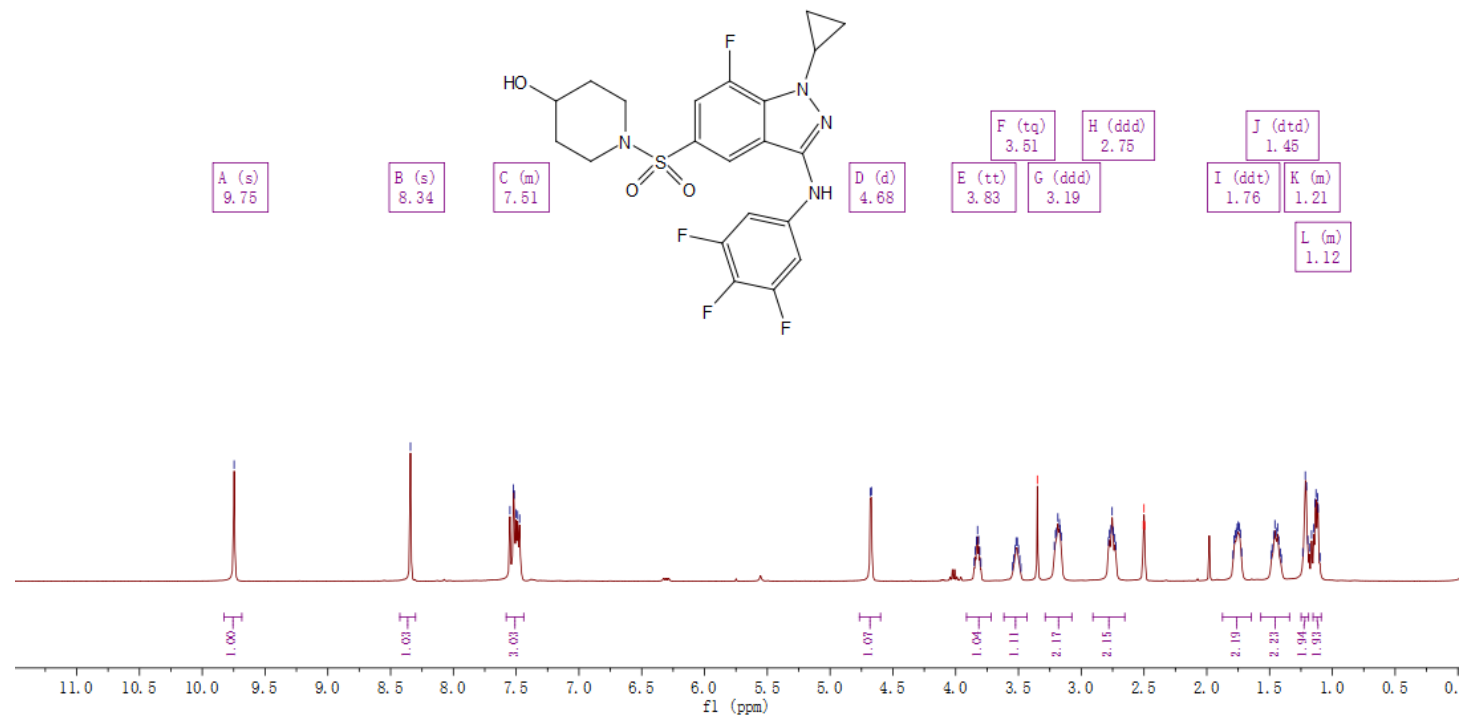

$20190306-$ wct $-1165-1-\mathrm{C} / 12$

C13CPD DUSO [D: \NMP

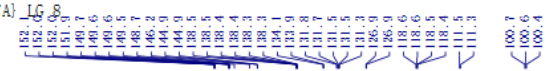

${ }^{13} \mathrm{C}$ NMR (101 MHz, DMSO- $\left.d_{6}\right) \delta 150.80$ (ddd, $\left.J=242.8,10.0,6.0 \mathrm{~Hz}\right), 147.46$ (d, $J=252.4 \mathrm{~Hz}$ ),

$144.92(\mathrm{~d}, J=2.2 \mathrm{~Hz}), 138.64-138.17(\mathrm{~m}), 134.35-131.49(\mathrm{~m}), 131.39(\mathrm{~d}, J=13.6 \mathrm{~Hz}), 126.93(\mathrm{~d}, J=$
$4.4 \mathrm{~Hz}), 118.62(\mathrm{~d}, J=6.0 \mathrm{~Hz}), 118.45(\mathrm{~d}, J=3.7 \mathrm{~Hz}), 111.37(\mathrm{~d}, J=20.7 \mathrm{~Hz}), 100.53(\mathrm{~d}, J=24.9 \mathrm{~Hz})$

$64.24,43.75,33.38,31.97(\mathrm{~d}, J=2.6 \mathrm{~Hz}), 7.81(\mathrm{~d}, J=3.0 \mathrm{~Hz})$.
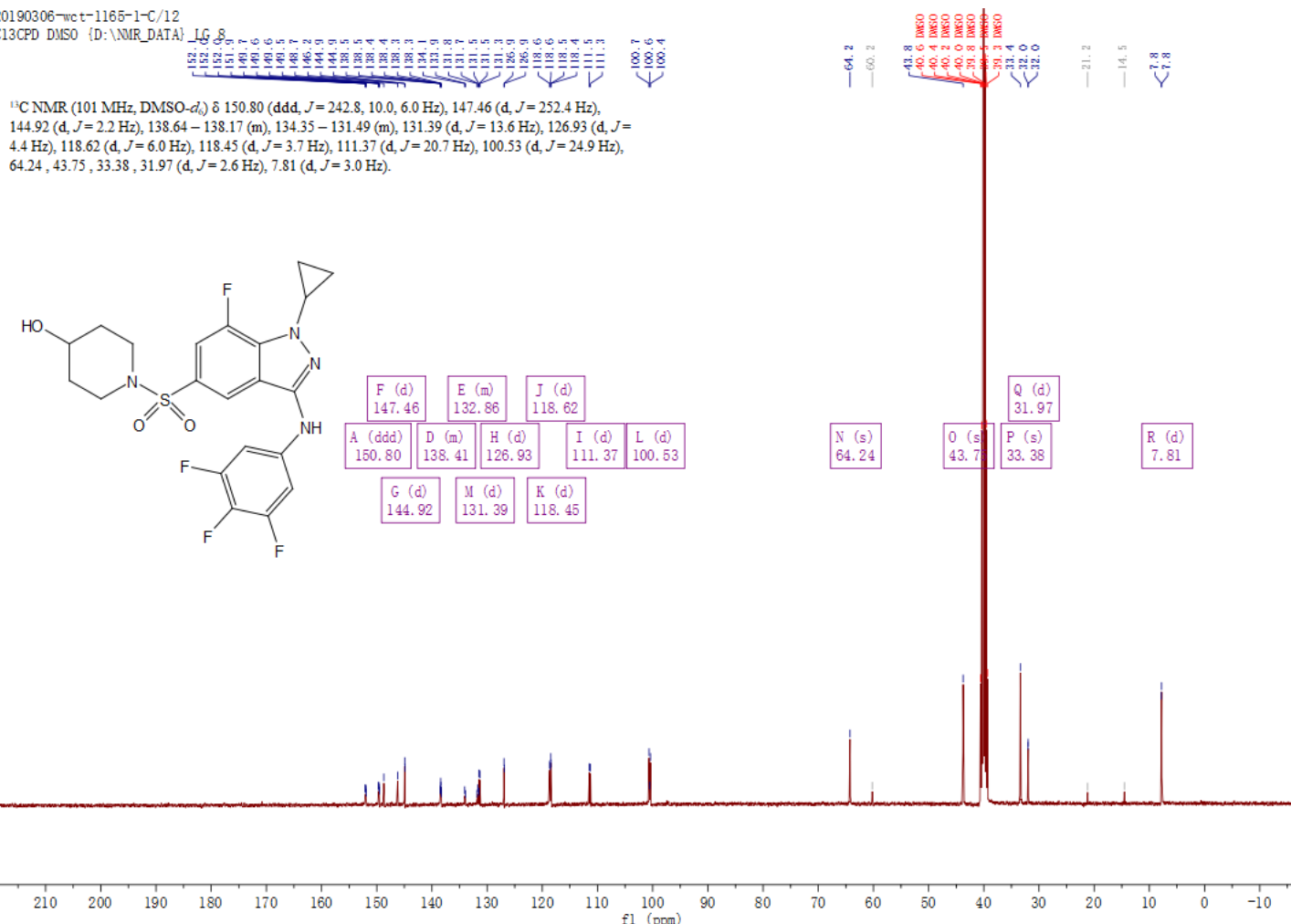


\section{Compound 8.}

20190530 wc t-1168-1-H/10
PROTON DUSO [D: $\backslash$ NMR_DATA\} LG 43

요요료 을

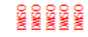

'H NMR (400 MHz, DMSO-d $\left.d_{\text {l }}\right) \delta 9.81$ (s, $\left.1 \mathrm{H}\right), 8.59$ (d, $\left.J=6.6 \mathrm{~Hz}, 1 \mathrm{H}\right), 7.60$ (d, $\left.J=11.2 \mathrm{~Hz}, 1 \mathrm{H}\right), 7.53$ (dd, $J=11.0,6.2 \mathrm{~Hz}, 2 \mathrm{H}), 4.71(\mathrm{~d}, J=4.0 \mathrm{~Hz}, 1 \mathrm{H}), 3.66(\mathrm{p}, J=5.3 \mathrm{~Hz}, 1 \mathrm{H}), 3.62-3.51(\mathrm{~m}, 1 \mathrm{H}), 2.89$

(ddd, $J=12.0,8.3,3.2 \mathrm{~Hz}, 2 \mathrm{H}), 1.76(\mathrm{ddc}, J=13.4,7,1,3.5 \mathrm{~Hz}, 2 \mathrm{H}), 1.43(\mathrm{ddd}, J=12.5,8.3,3.7 \mathrm{~Hz}$<smiles>Cn1nc(Nc2cc(F)c(F)c(F)c2)c2cc(S(=O)(=O)N3CCCCC3)c(F)cc21</smiles><smiles>C1=CCCCC1</smiles><smiles>CCCCC</smiles>

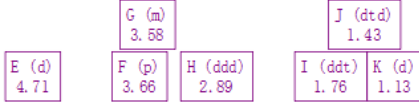

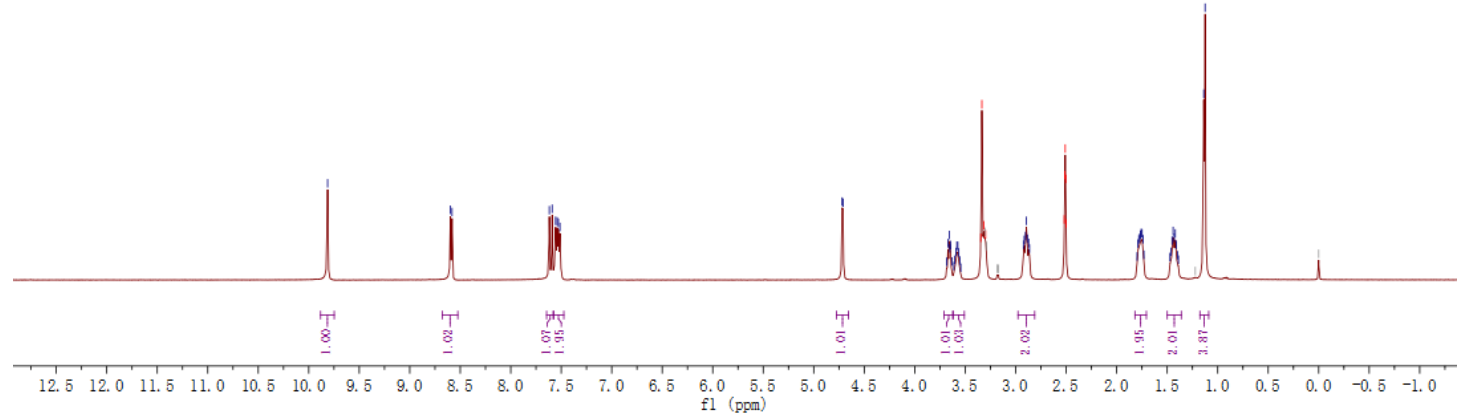

20190530-wCt-1168-1-C/10
C13CPD DUSO \{D: \NMR_DATA\} LG 53

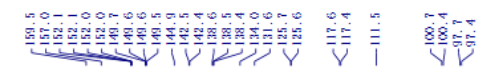

${ }^{13} \mathrm{C} \mathrm{NMR}\left(101 \mathrm{MHz}\right.$, DMSO-d $\left.d_{\mathrm{s}}\right) \delta 158.26$ (d, $J=251.3 \mathrm{~Hz}$ ), 150.83 (ddd $J=243.0,10.1,6.4 \mathrm{~Hz}$ ),

$144.93,142.45(\mathrm{~d}, J=12.5 \mathrm{~Hz}), 138.52(\mathrm{t}, J=12.6 \mathrm{~Hz}), 132.84(\mathrm{~d}, J=240.9 \mathrm{~Hz}), 125.64(\mathrm{~d}, J=3.1 \mathrm{~Hz})$,

$29.82,6.83$.<smiles>O=S(=O)(c1cc2c(Nc3cc(F)c(F)c(F)c3)nn(C3CC3)c2cc1F)N1CCC(O)CC1</smiles> 


\section{Compound 9.}

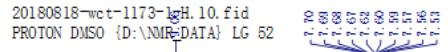
Fonspas

'H NMR (400 MHz, DMSO- $\left.d_{6}\right) \delta 9.06(\mathrm{~s}, 1 \mathrm{H}), 7.68$ (dd, $\left.J=8.9,6.2 \mathrm{~Hz}, 1 \mathrm{H}\right), 7.59$ (dd, $J=11.2,6.3 \mathrm{~Hz}$ $2 \mathrm{H}), 7.55(\mathrm{~d}, J=8.9 \mathrm{~Hz}, 1 \mathrm{H}), 4.71(\mathrm{~d}, J=4.0 \mathrm{~Hz}, 1 \mathrm{H}), 3.73(\mathrm{tt}, J=7.0,4.0 \mathrm{~Hz}, 1 \mathrm{H}), 3.56(\mathrm{dq}, J=8.1,4$. (dtd, $J=12.3,8.1,3.7 \mathrm{~Hz}, 2 \mathrm{H}), 1.20-1.11(\mathrm{~m}, 4 \mathrm{H})$.

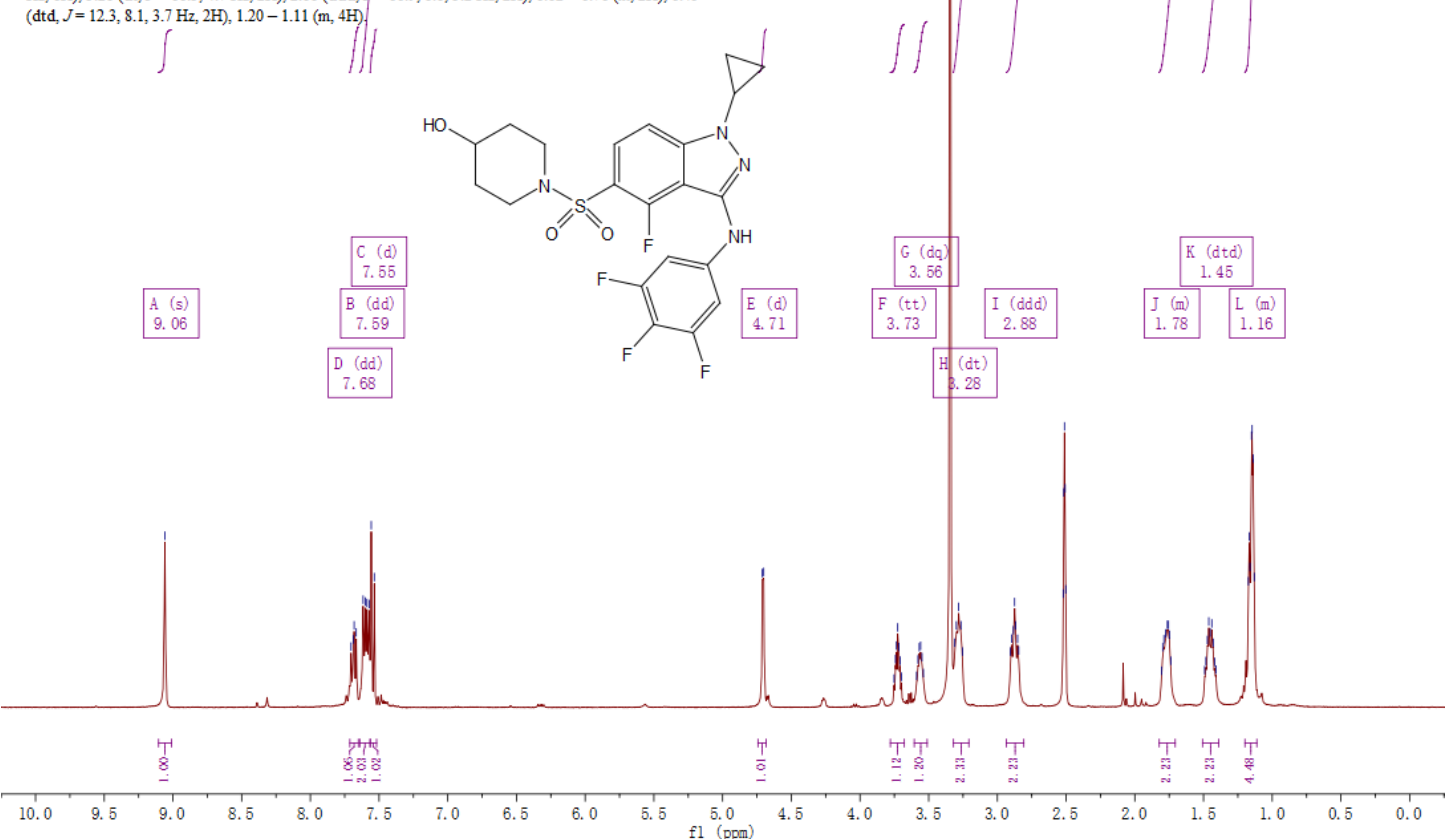

Desktop. 11. fid
C13CPD DISO $\{$ D: $\backslash$ NIR_DATA $\}$ LG 52

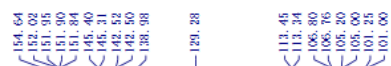

${ }^{13} \mathrm{CNMR}\left(101 \mathrm{MHz}, \mathrm{DMSO}-d_{\mathrm{s}}\right) \delta 153.33(\mathrm{~d}, J=263.6 \mathrm{~Hz}), 151.99-149.03(\mathrm{~m}), 145.36(\mathrm{~d}, J=8.7 \mathrm{~Hz})$ $142.51(\mathrm{~d}, J=2.2 \mathrm{~Hz}), 138.98,129.28,113.39(\mathrm{~d}, J=11.3 \mathrm{~Hz}), 106.78(\mathrm{~d}, J=3.9 \mathrm{~Hz}), 105.10$ (d, $J=$

$20.1 \mathrm{~Hz}), 101.13(\mathrm{~d}, J=24.9 \mathrm{~Hz}), 64.31,43.47,33.48,29.94,6.89$
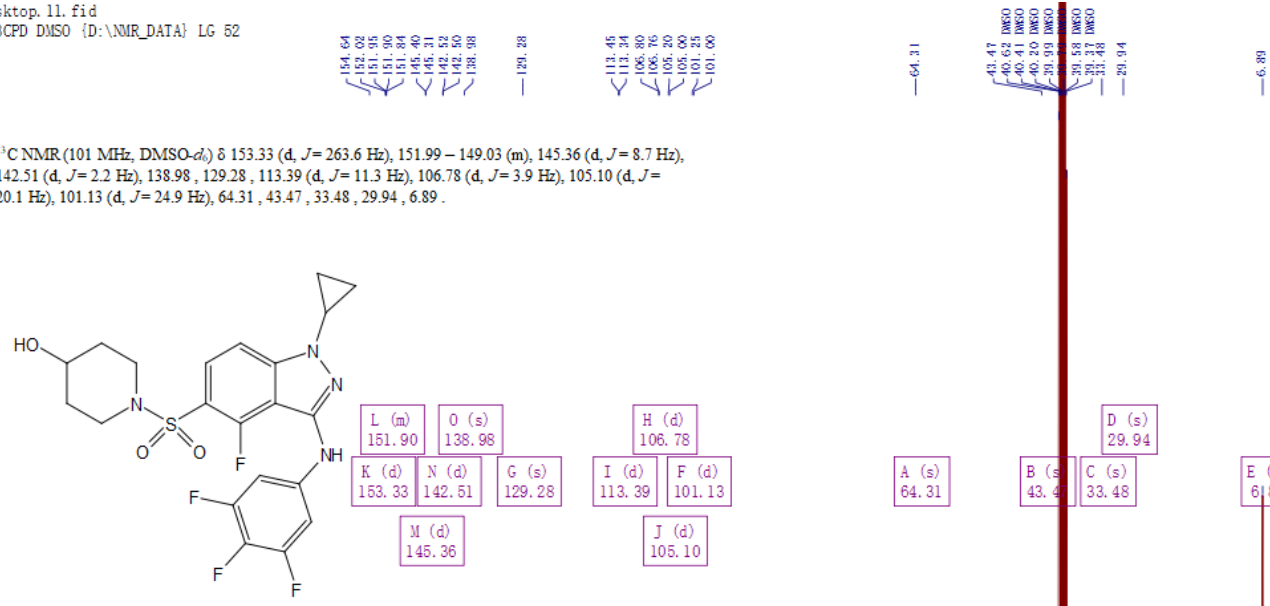


\section{Compound 10.}

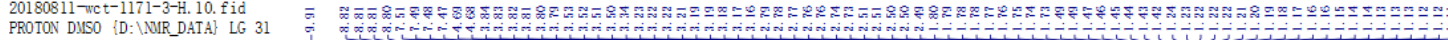

'H NMR ( $400 \mathrm{MHz}$, DMSO- $\left.d_{6}\right) \delta 9.91(\mathrm{~s}, 1 \mathrm{H}), 8.82(\mathrm{~d}, J=2.1 \mathrm{~Hz}, 1 \mathrm{H}), 8.80(\mathrm{~d}, J=2.1 \mathrm{~Hz}, 1 \mathrm{H}), 7.49$

$\mathrm{Hz}, 1 \mathrm{H}$ ), 3.20 (ddd, $J=13.1,6.1,3.0 \mathrm{~Hz}, 2 \mathrm{H}$ ), 2.76 (ddd, $J=11.8,8.6,3.3 \mathrm{~Hz}, 2 \mathrm{H}$ ) 1.77 (ddt, $J=13.5$

$6.6,3.5 \mathrm{~Hz}, 2 \mathrm{H}), 1.45(\mathrm{dtd}, J=12.3,8.0,3.5 \mathrm{~Hz}, 2 \mathrm{H}), 1.25-1.18(\mathrm{~m}, 2 \mathrm{H}), 1.18-1.10(\mathrm{~m}, 2 \mathrm{H})$

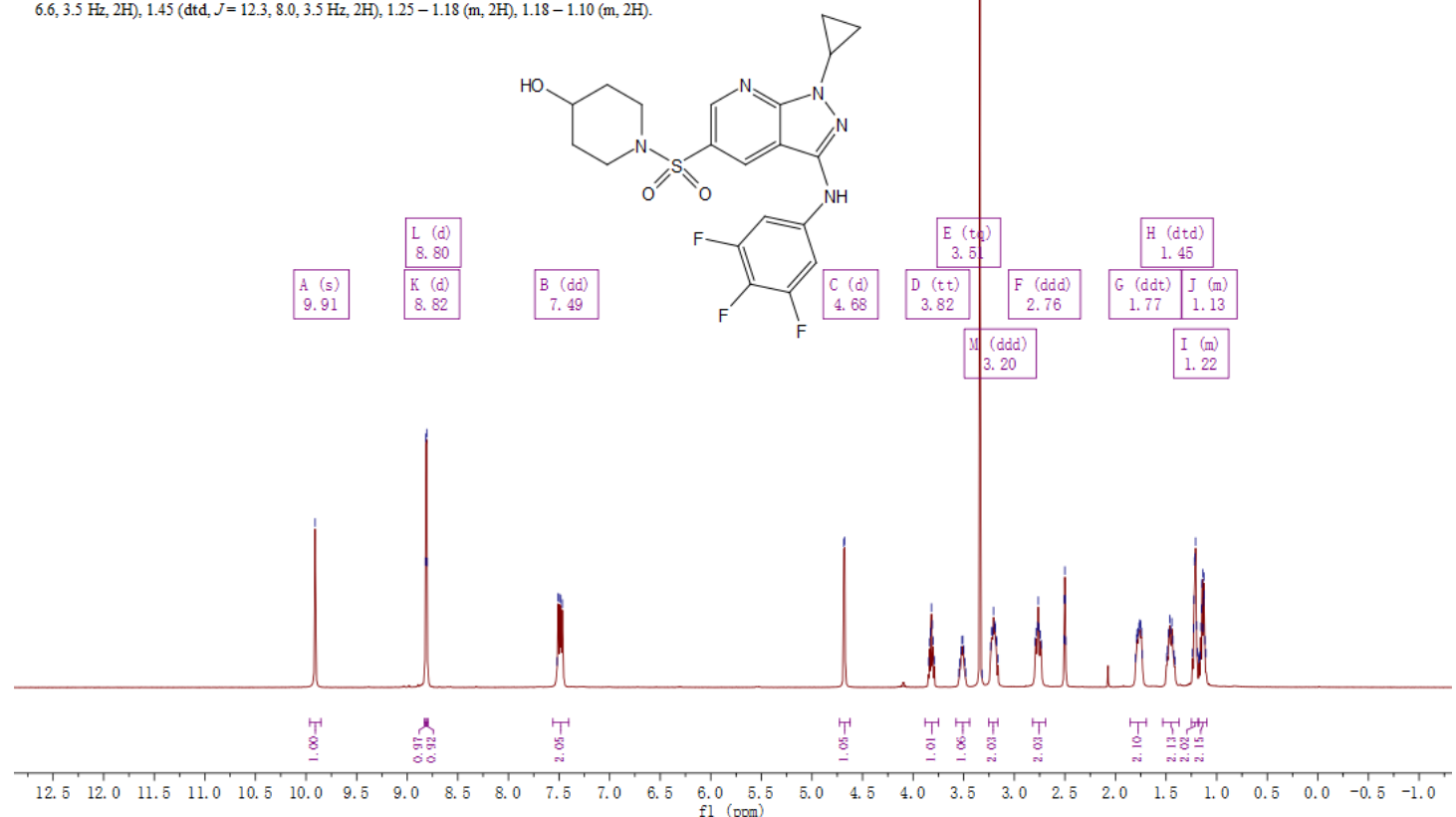

20180811-wct-1173-3-C. 10, fid
C13CPD DUSO \{D: \NMIR_DATA\} LG 3.

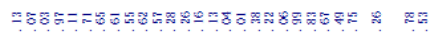

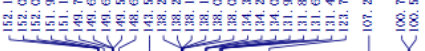

${ }^{13} \mathrm{C} \mathrm{NMR}\left(101 \mathrm{MHz}, \mathrm{DMSO}-d_{\mathrm{c}}\right.$ ) $\delta 151.11,150.84$ (ddd, $J=243.0,10.0,5.8 \mathrm{~Hz}$ ), $148.62,143.57,138.15$ (td, $J=12.3,2.8 \mathrm{~Hz}), 133.02$ (dt $J=240.5,15.9 \mathrm{~Hz}), 131.49,123.75,107.26,100.66$ (d, $J=24.3 \mathrm{~Hz}$ ), $64.24,43.69,33.37,29.17,6.61$<smiles>O=S(=O)(c1cnc2c(c1)c(Nc1cc(F)c(F)c(F)c1)nn2C1CC1)N1CCC(O)CC1</smiles>

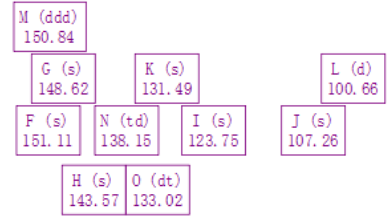




\section{Compound 11.}

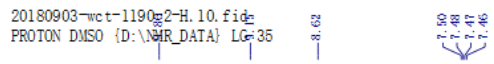
$2 \mathrm{H}), 4.69(\mathrm{~d}, J=3.9 \mathrm{~Hz}, 1 \mathrm{H}), 3.84(\mathrm{dq}, J=6.9,3.5,3.0 \mathrm{~Hz}, 1 \mathrm{H}), 3.63-3.48(\mathrm{~m}, 2 \mathrm{H}), 3.41(\mathrm{dt}, J=11.4$,
$4.5 \mathrm{~Hz}, 2 \mathrm{H}), 2.94(\mathrm{ddd}, J=12.2 .8 .8,3.4 \mathrm{~Hz}, 2 \mathrm{H}), 1.83-1.67(\mathrm{~m}, 2 \mathrm{H}), 1.52-1.33(\mathrm{~m}, 2 \mathrm{H}), 1.23-1.16$ $(\mathrm{m}, 4 \mathrm{H})$

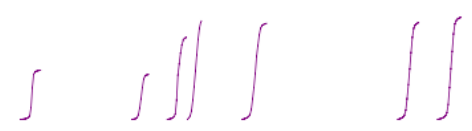

(s)
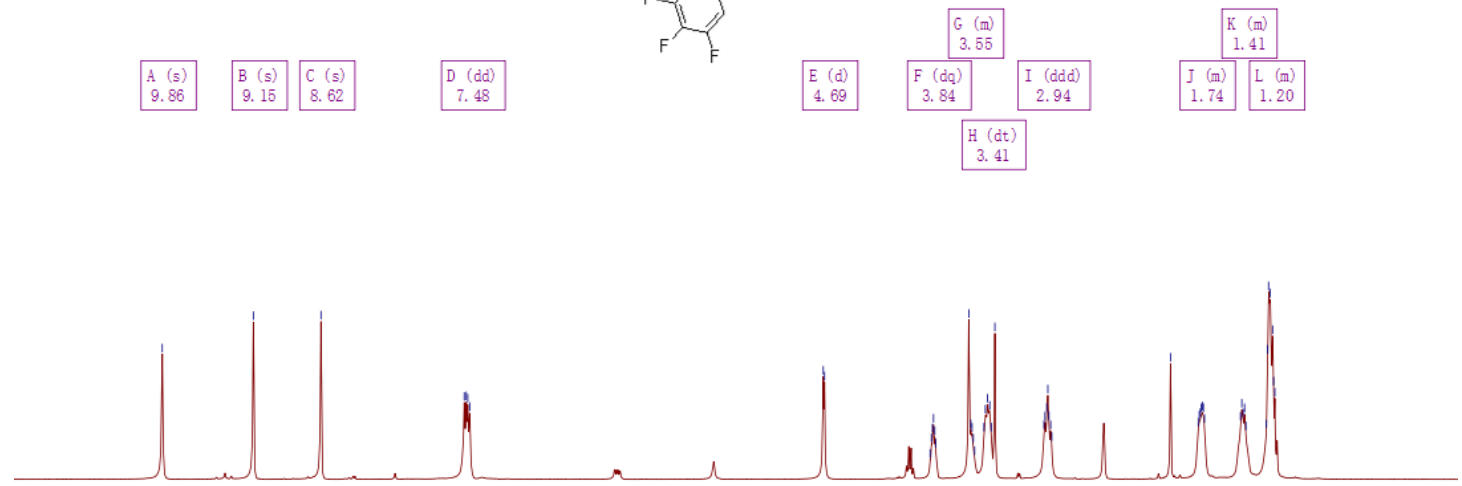

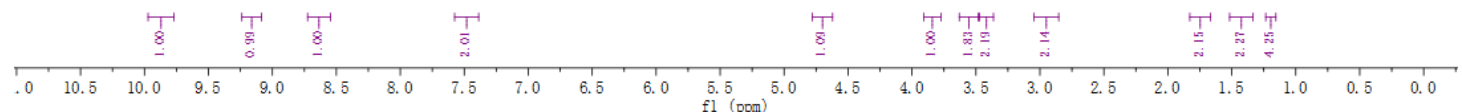

20180903-wct-1190-2-C. 12. fid

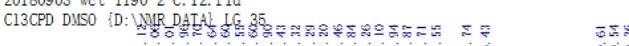

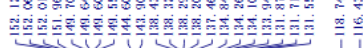

-580
$\frac{88}{4}$

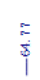

${ }^{13} \mathrm{CNMR}\left(101 \mathrm{MHz}, \mathrm{DMSO}-d_{k}\right.$ ) $\delta 150.83$ (ddd, $J=242.6,10.0,5.8 \mathrm{~Hz}$ ), 144.60, 143.90, $138.94-$

$137.93(\mathrm{~m}), 137.46,134.84,132.90(\mathrm{~d}, J=240.7 \mathrm{~Hz}), 118.74,116.43,100.48(\mathrm{~d}, J=24.7 \mathrm{~Hz}), 64.77$ $44.16,33.76,30.30,6.94$.

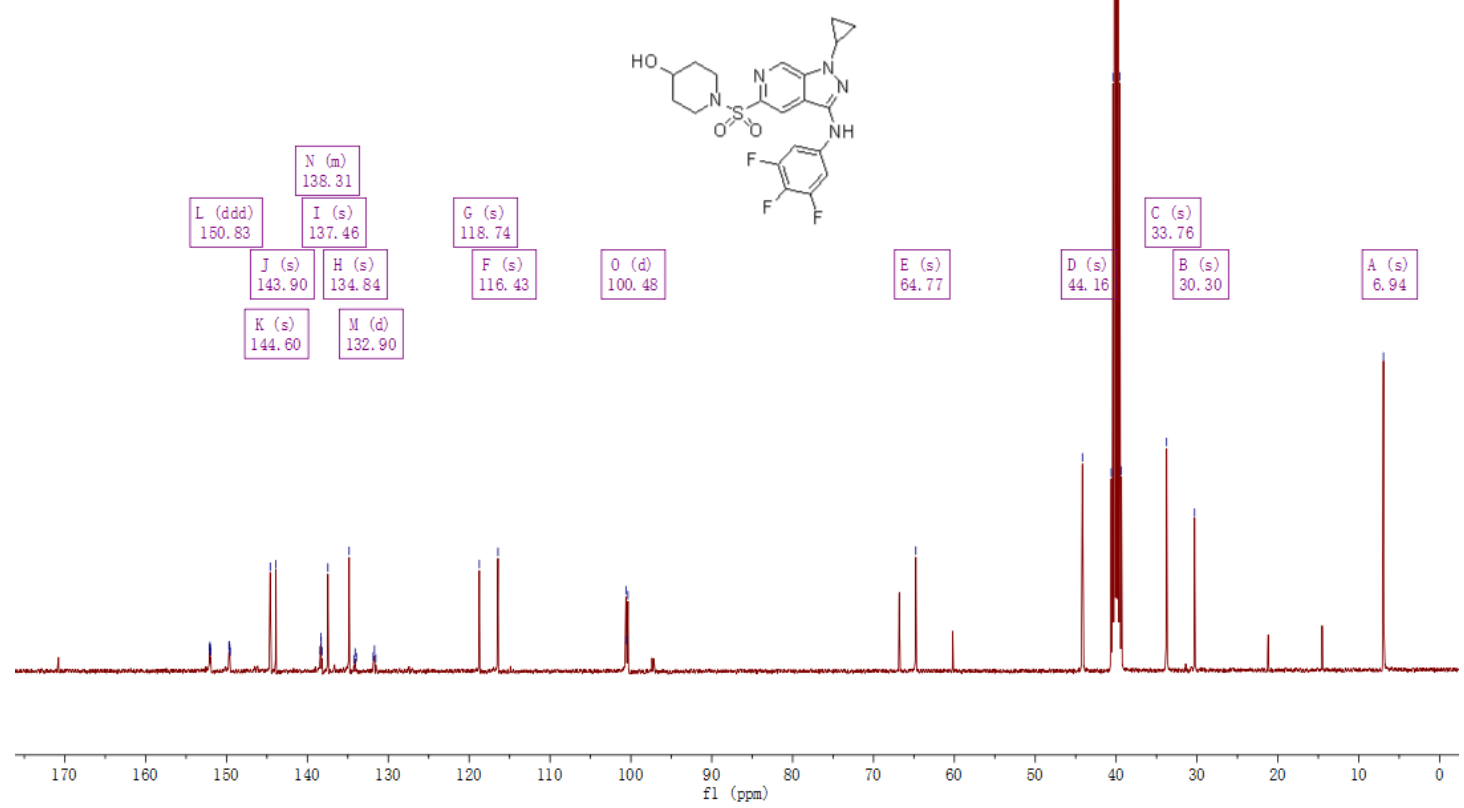




\section{Compound 12.}

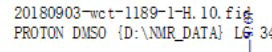

'H NMR (400 MHz, DMSO- $\left.d_{9}\right) 89.67(\mathrm{~s}, 1 \mathrm{H}), 8.25(\mathrm{~d}, J=8.8 \mathrm{~Hz}, 1 \mathrm{H}), 7.94(\mathrm{~d}, J=8.8 \mathrm{~Hz}, 1 \mathrm{H}), 7.72$ $(\mathrm{dd}, J=11.2,6.2 \mathrm{~Hz}, 2 \mathrm{H}), 4.69$ (d, $J=4.0 \mathrm{~Hz}, 1 \mathrm{H}), 3.81-3.67(\mathrm{~m}, 1 \mathrm{H}), 3.59-3.46(\mathrm{~m}, 1 \mathrm{H}), 3.41$ (dd, $J$

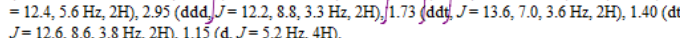<smiles>CC=CCCC</smiles>
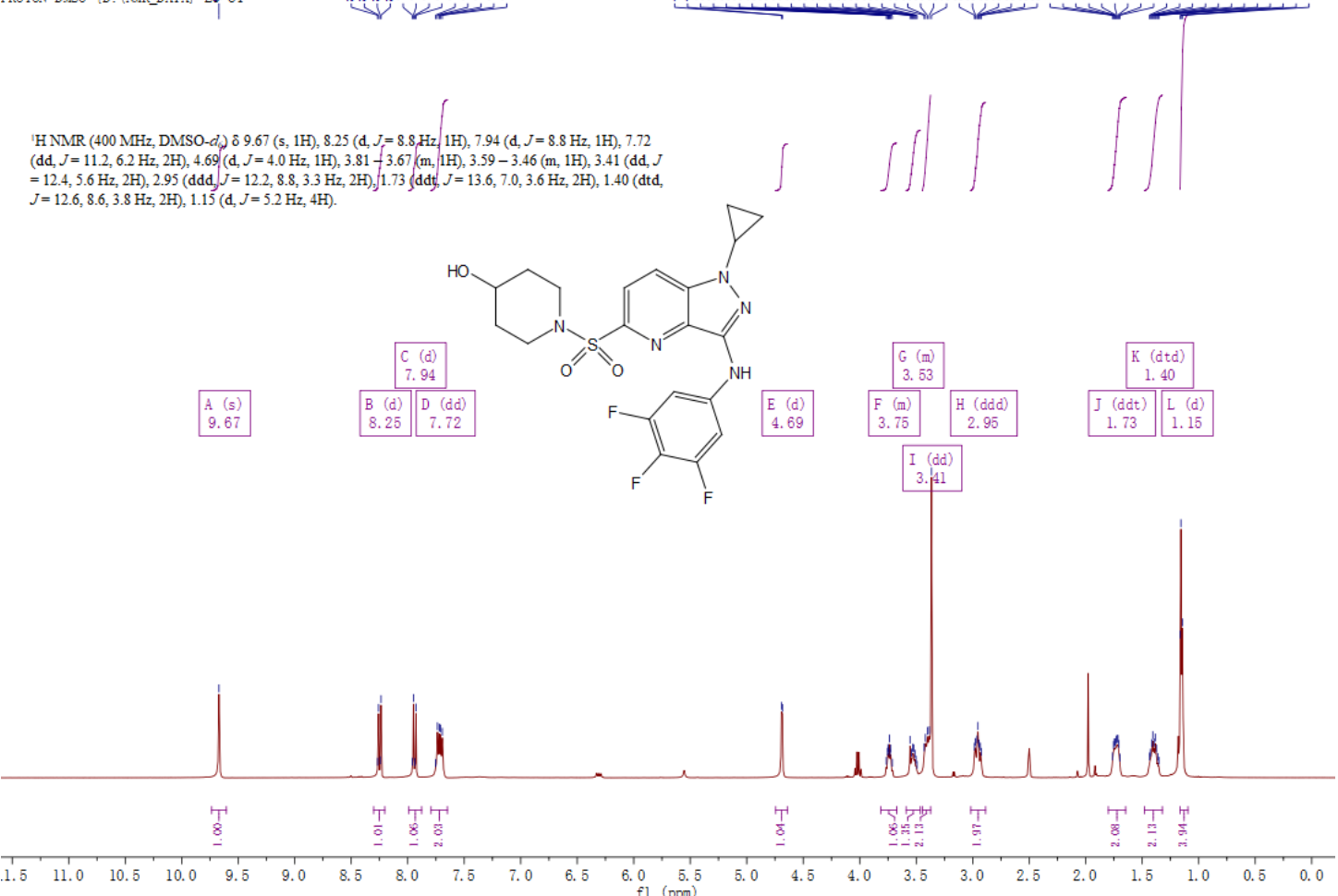

20190627- wct-1189-1-C/10

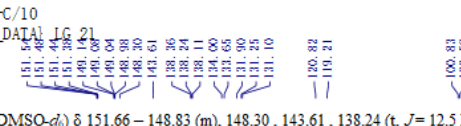

$\frac{8}{\mathrm{z}}$

${ }^{13} \mathrm{CNMR}\left(101 \mathrm{MHz}, \mathrm{DMSO}-d_{\mathrm{s}}\right) \delta 151.66-148.83(\mathrm{~m}), 148.30,143.61,138.24(\mathrm{t}, J=12.5 \mathrm{~Hz}), 134.00$
$133.88-131.08(\mathrm{~m}), 131.90,120.82,119.21,100.70$ (d, $J=24.7 \mathrm{~Hz}), 64.19,43.59,33.25,29.57$,

6.21.
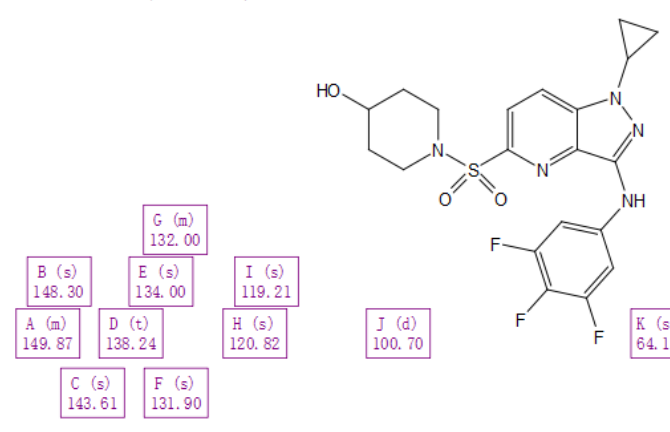


\section{Compound 13.}

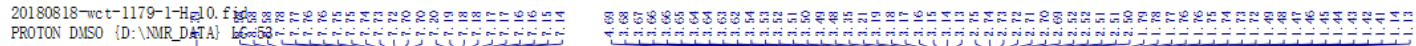

'H NMR $\left(400 \mathrm{MHz}\right.$, DMSO- $\left.d_{\mathrm{d}}\right) \delta 9.39(\mathrm{~s}, 1 \mathrm{H}), 8.58(\mathrm{~d}, J=1.3 \mathrm{~Hz}, 1 \mathrm{H}), 7.79-7.72(\mathrm{~m}, 2 \mathrm{H}), 7.70(\mathrm{~d}, J$

$=1.2 \mathrm{~Hz}, 2 \mathrm{H}), 7.21-7.13(\mathrm{~m}, 2 \mathrm{H}), 4.69(\mathrm{~s}, 1 \mathrm{H}), 3.65(\mathrm{p}, J=5.3 \mathrm{~Hz}, 1 \mathrm{H}), 3.51(\mathrm{tt}, J=7.6,3.6 \mathrm{~Hz}, 1 \mathrm{H})$,

$3.18-3.11(\mathrm{~m}, 2 \mathrm{H}), 2.72$ (ddd, $J=11.8,8.4,3.4 \mathrm{~Hz}, 2 \mathrm{H}$ ), 1.76 (ddt, $J=13.5,6.8,2.9 \mathrm{~Hz}, 2 \mathrm{H}$ ), 1.45 (dtd,

$J=12.0,7.8,3.6 \mathrm{~Hz}, 2 \mathrm{H}), 1.13(\mathrm{~d}, J=5.2 \mathrm{~Hz}, 4 \mathrm{H})$.
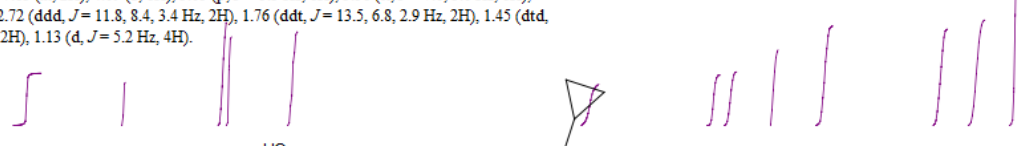

HOY<smiles>CNc1nn(C)c2ccc(S(=O)(=O)N3CCCCC3)cc12</smiles>

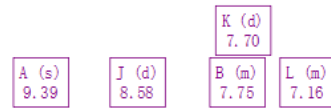
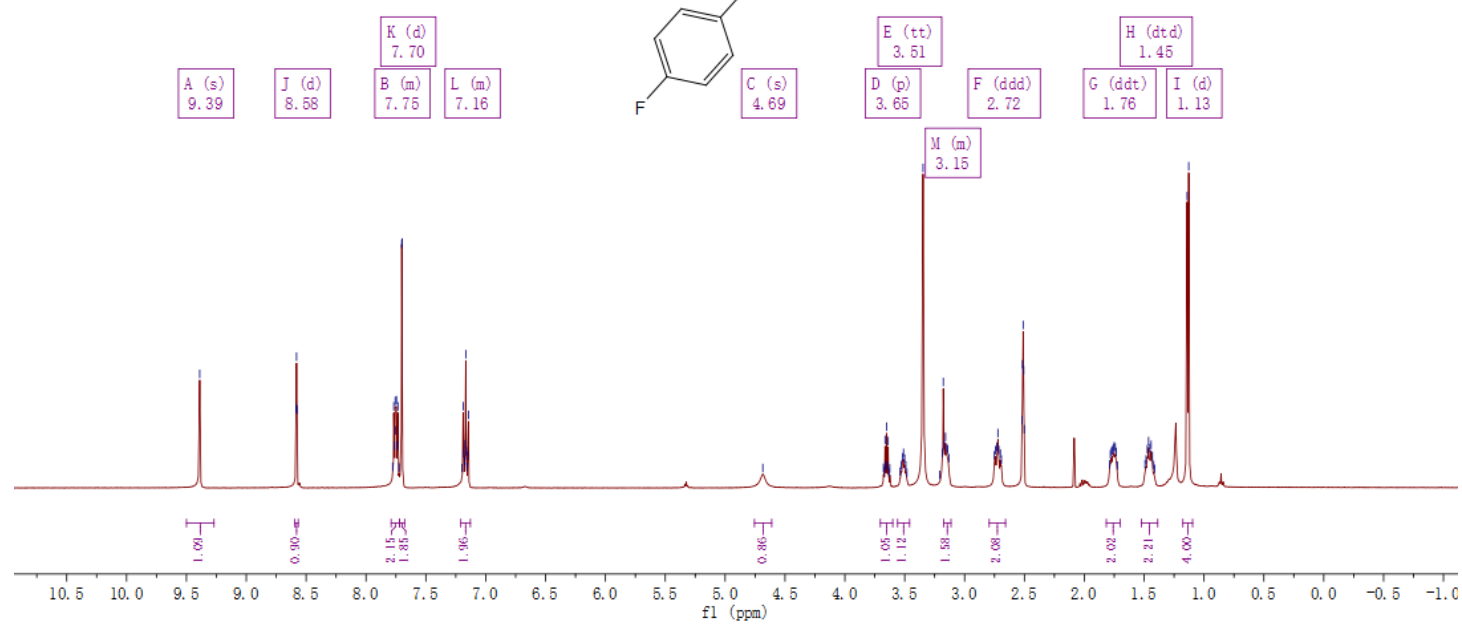

20180818-wct-1179-1-C. 20. fid

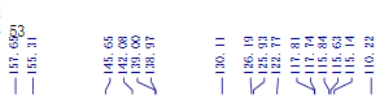

${ }^{13} \mathrm{CNMR}\left(101 \mathrm{MHz}, \mathrm{DMSO}-d_{\mathrm{d}}\right) \delta 156.48$ (d, $\left.J=235.7 \mathrm{~Hz}\right), 145.65,142.08,138.99$ (d, $\left.J=2.1 \mathrm{~Hz}\right), 126.06$ (d, $J=26.0 \mathrm{~Hz}), 122.77,117.78(\mathrm{~d}, J=7.4 \mathrm{~Hz}), 115.84,115.63,115.14,110.22,64.25,43.72,33.37,29.57,6.87$.

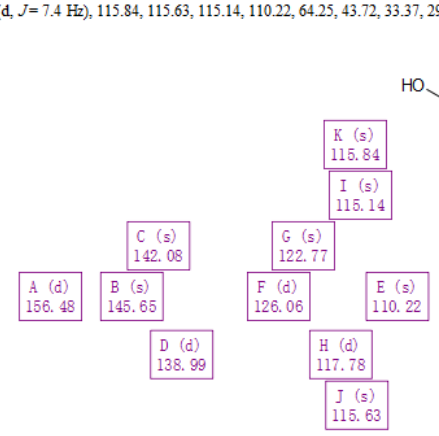

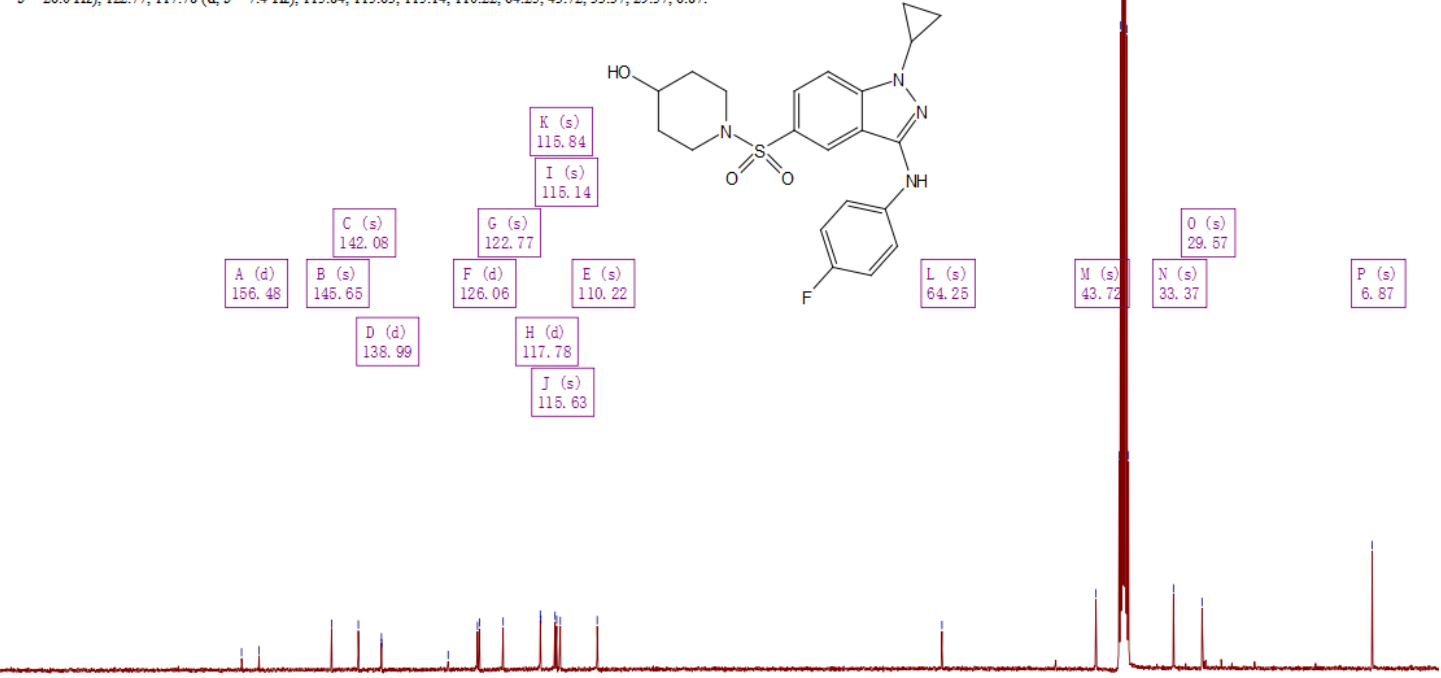

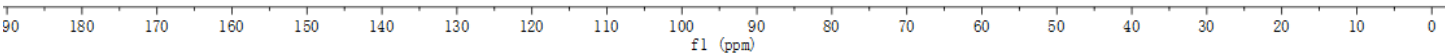




\section{Compound 14.}

20180924-wct-1199-1-H. 10, fid
PROTON DISO \{D: LNIR_DATA\} LG 46

8 .

增

IH NMR (400 MHz, DMSO- $\left.d_{\mathrm{s}}\right) \delta 9.24(\mathrm{~s}, 1 \mathrm{H}), 8.60(\mathrm{t}, J=1.3 \mathrm{~Hz}, 1 \mathrm{H}), 7.72-7.67(\mathrm{~m}, 2 \mathrm{H}), 7.67-7.61$

$1 \mathrm{H}), 3.16$ (ddd, $J=11.1,6.9,3.7 \mathrm{~Hz}, 2 \mathrm{H}), 2.72(\mathrm{ddd}, J=11.8,8.4,3.4 \mathrm{~Hz}, 2 \mathrm{H}), 2.26(\mathrm{~s}, 3 \mathrm{H}), 1.76$ (ddt, $J$

$=13.5,6.8,2.9 \mathrm{~Hz}, 2 \mathrm{H}), 1.46(\mathrm{dtd}, J=12.0,7.9,3.5 \mathrm{~Hz}, 2 \mathrm{H}), 1.13(\mathrm{~d}, J=5.2 \mathrm{~Hz}, 4 \mathrm{H})$.

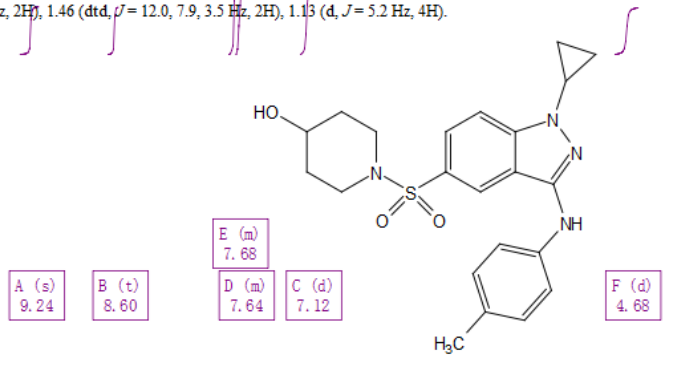

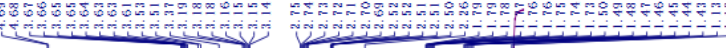

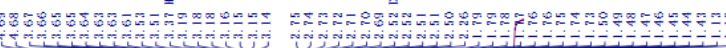

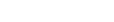

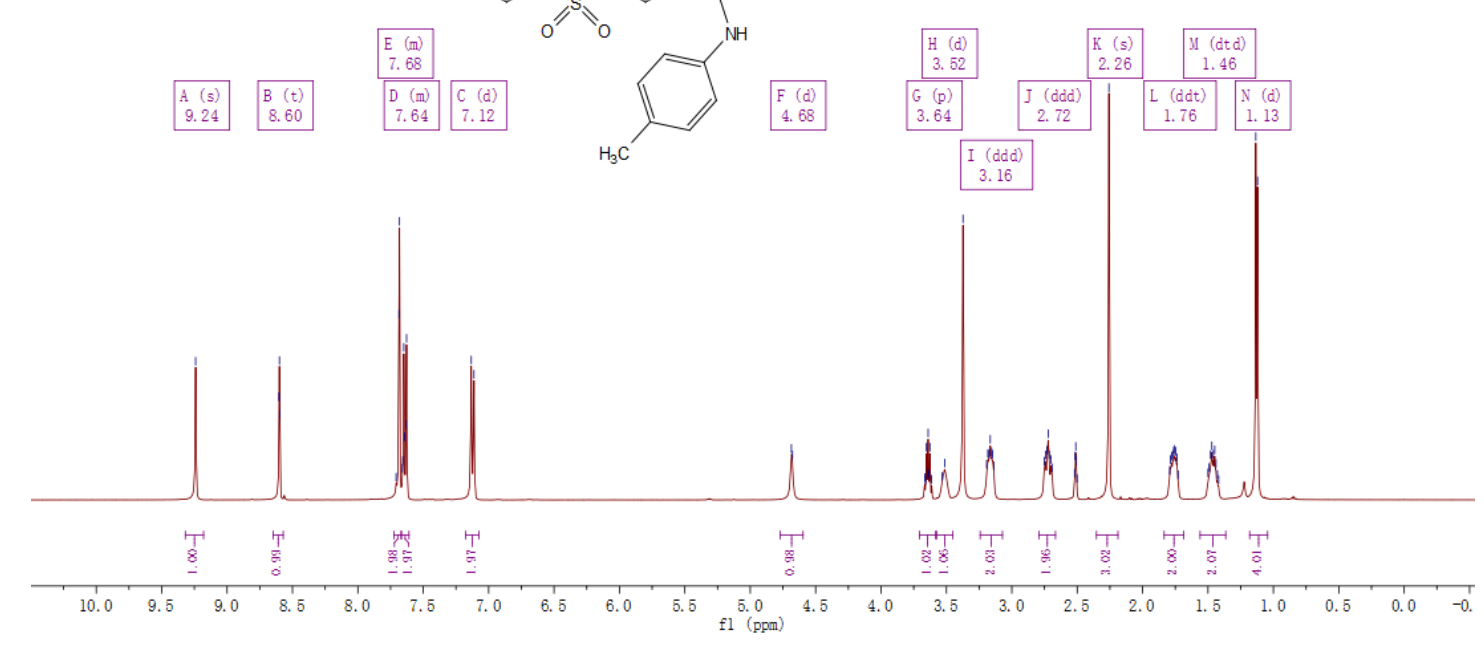

20180924-wct-1199-1-C. 20, fid
C13CPD DUSO \{D: $\backslash$ NIR_DATA\} LG 4

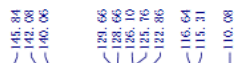

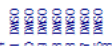

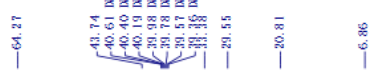

${ }^{13} \mathrm{C} \mathrm{NMR}\left(101 \mathrm{MHz}\right.$, DMSO- $\left.d_{l}\right) \delta 145.84,142.08,140.06,129.66,128.66,126.10,125.76,122.86$ $116.64,115.31,110.08,64.27,43.74,33.38,29.55,20.81,6.86$
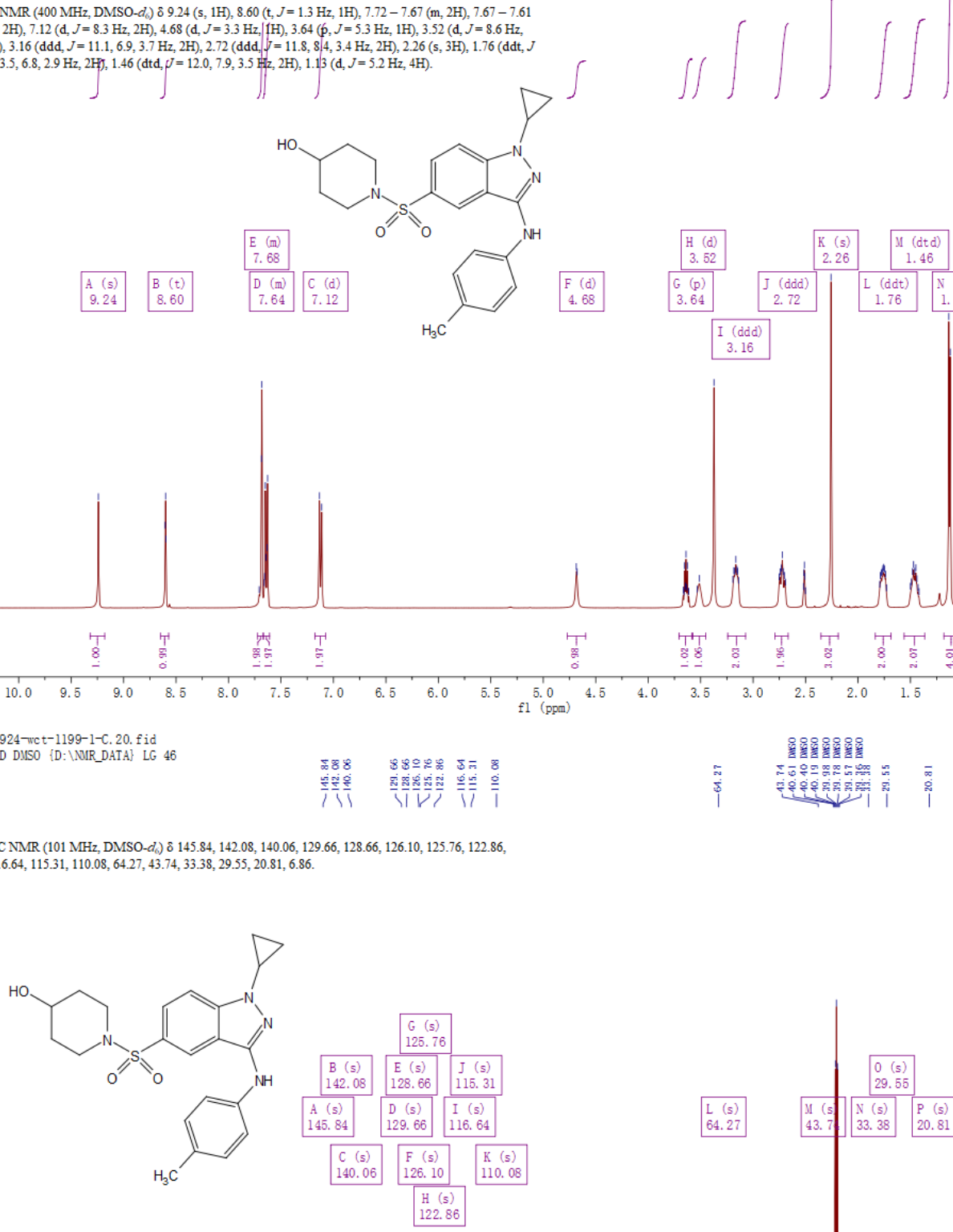


\section{Compound 16.}

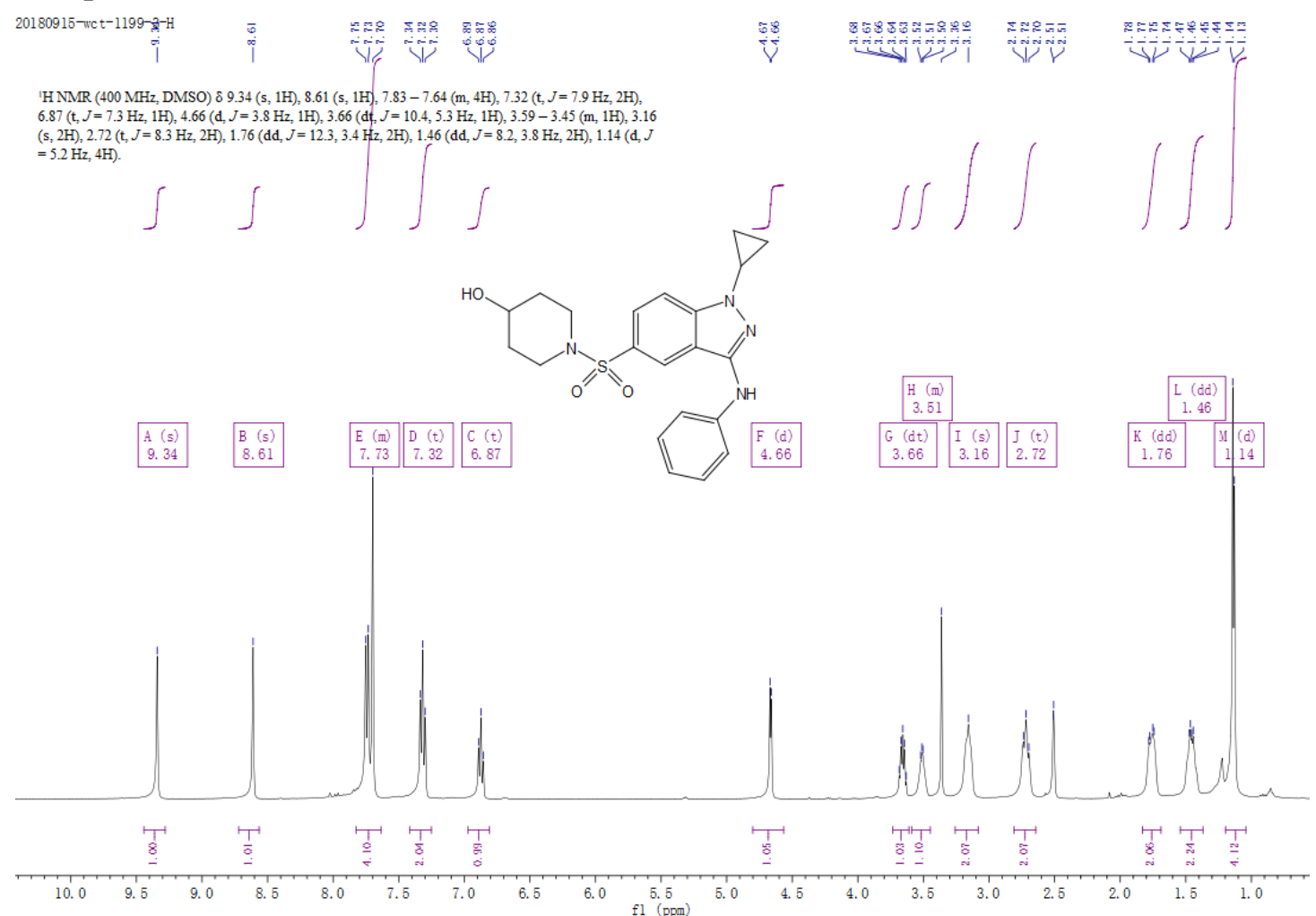

20180917-wct-1199-3-C. 10. fid

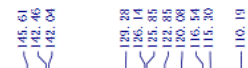

${ }_{13}^{13} \mathrm{CNMR}\left(101 \mathrm{MHz}, \mathrm{DMSO}-\mathrm{d}_{2}\right) \delta 145.61,142.46,142.04,129.28,126.14,125.85,122.85,120.08$, $116.54,115.30,110.19,64.25,43.74,33.37,29.58,6.88$.
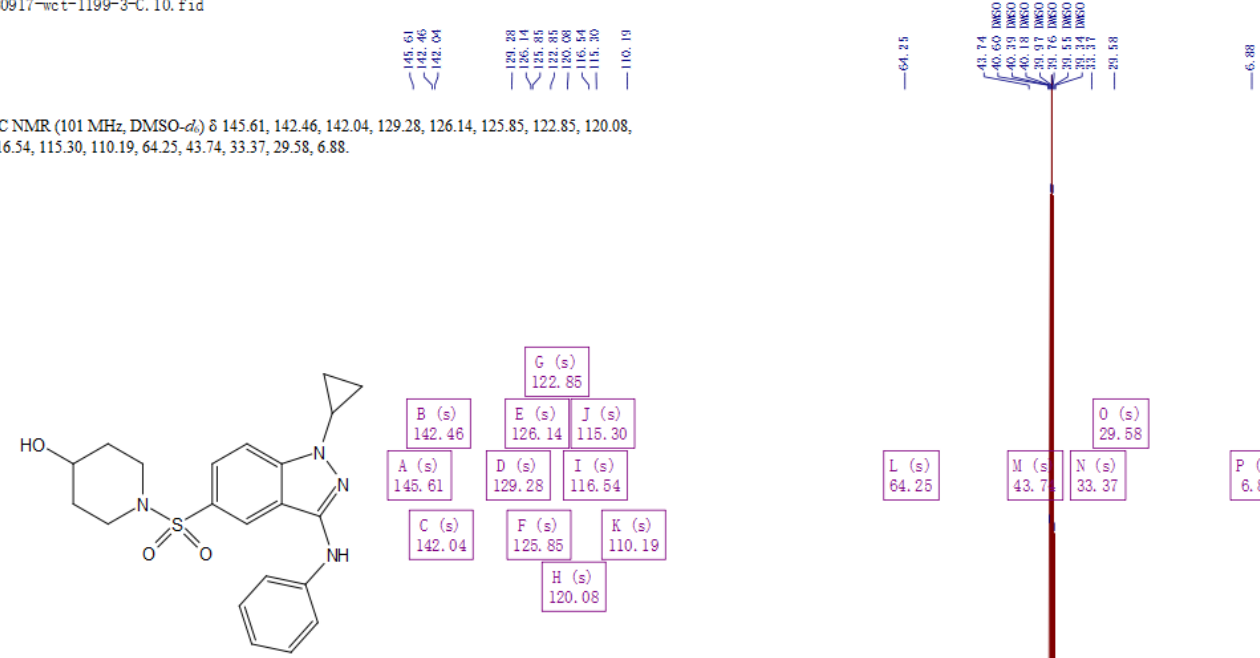


\section{Compound 17.}

20180924-wct-1202-1-H. 10, fid
PROTON DUSO \{D: $\backslash$ NMR_DATA\} LG

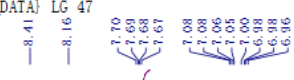

$\stackrel{8}{=}$

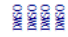

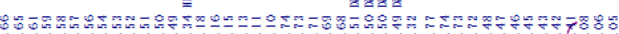

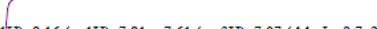

'H NMR (400 MHz, DMSO-d $\left.d_{\mathrm{s}}\right) \delta 8.41(\mathrm{~s}, 1 \mathrm{H}), 8.16(\mathrm{~s}, 1 \mathrm{H}), 7.81-7.61(\mathrm{~m}, 3 \mathrm{H}), 7.07$ (dd, $J=9.7,2.9$

$\mathrm{Hz}, 1 \mathrm{H}), 7.02-6.93(\mathrm{~m}, 1 \mathrm{H}), 4.65(\mathrm{~d}, J=3.8 \mathrm{~Hz}, 1 \mathrm{H}), 3.65-3.54(\mathrm{~m}, 1 \mathrm{H}), 3.54-3.45(\mathrm{~m}, 1 \mathrm{H}), 3.14(\mathrm{td}$

$J=11.1,10.7,5.0 \mathrm{~Hz}$,
$1.14-0.97(\mathrm{~m}, 4 \mathrm{H})$
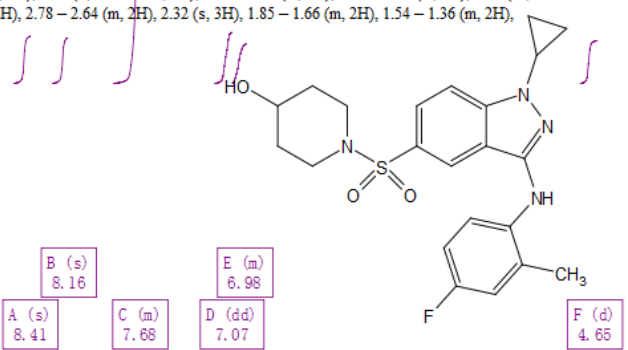

$\sqrt{-1}$

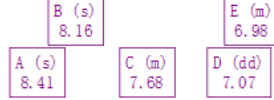
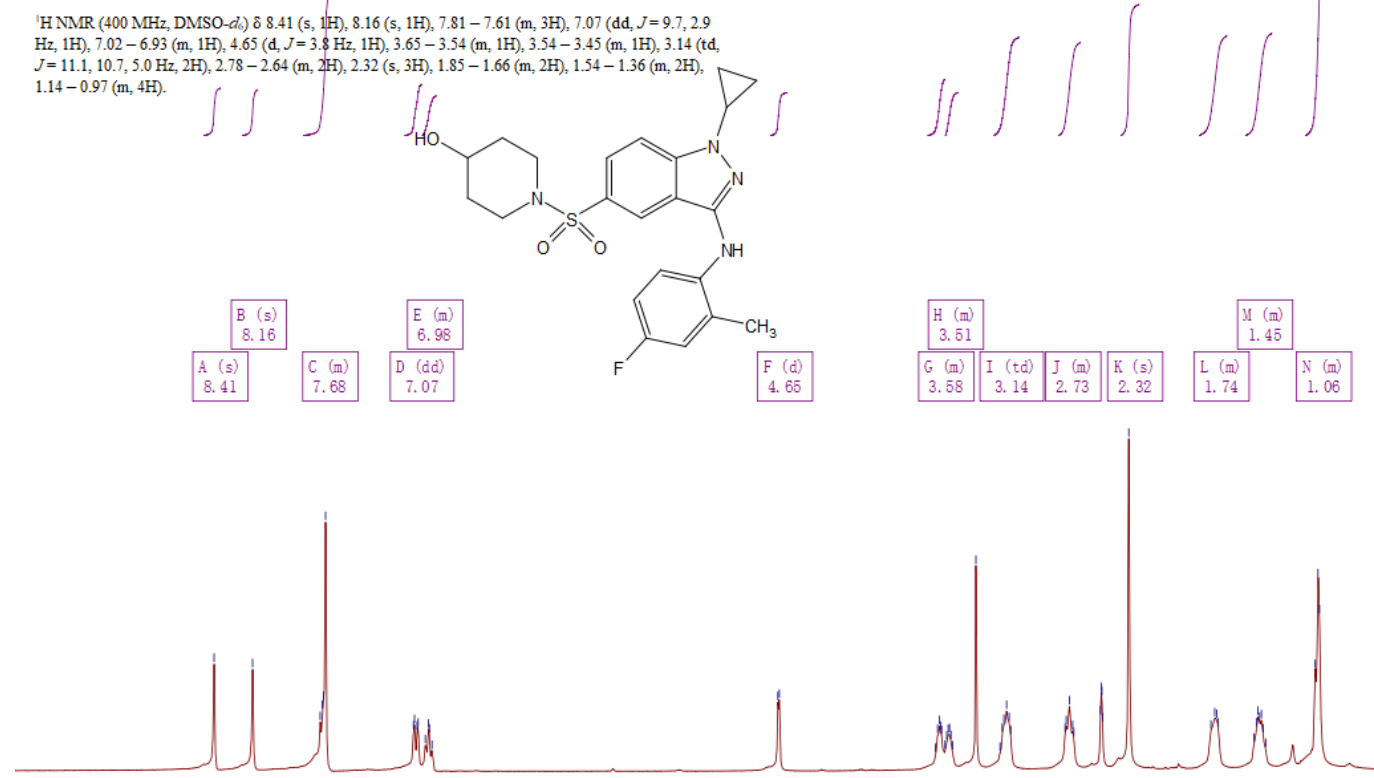

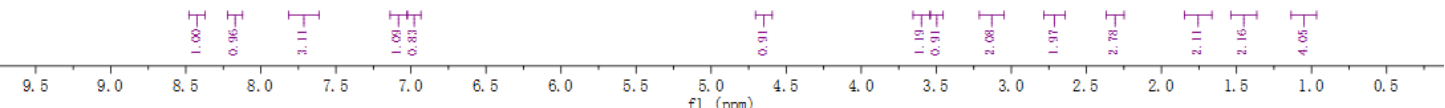

20180925-wct-1202-1-C. 10, fid
C13CPD DMSO [D: LNIR__ATA\} LG 2

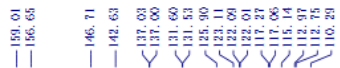

${ }^{13} \mathrm{CNMR}\left(101 \mathrm{MHz}, \mathrm{DMSO}-d_{\mathrm{s}}\right) \delta 157.83$ (d, $\left.J=237.9 \mathrm{~Hz}\right), 146.71,142.63,137.02$ (d, $J=2.5 \mathrm{~Hz}$ ),

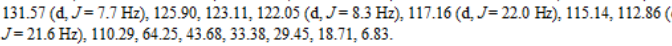

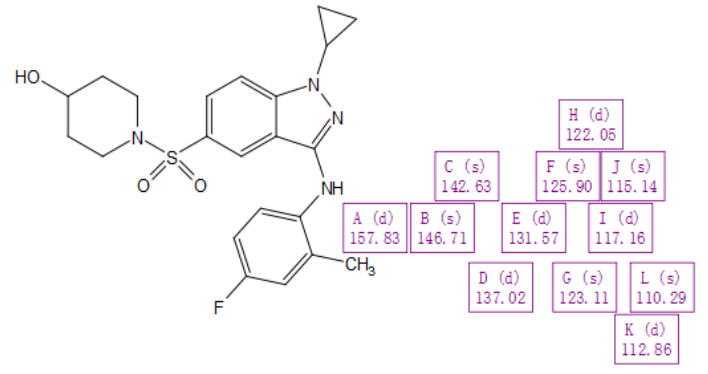

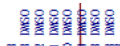

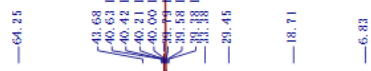
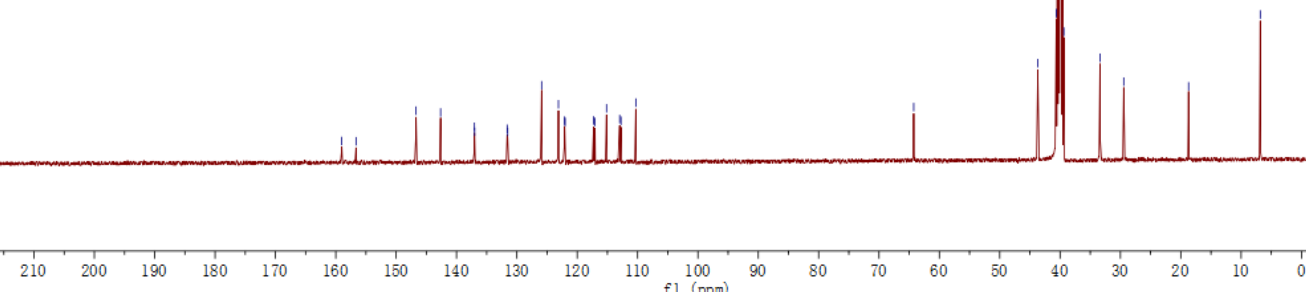


\section{Compound 18.}

$20180924-w c t-1202-2-$ H. 10, fid
PROTON DUISO \{D: INIR_DATA\} LG

'H NMR (400 MHz, DMSO-d $\left.d_{6}\right) \delta 8.57$ (s, $\left.1 \mathrm{H}\right), 854$ (d, $\left.J=1.5 \mathrm{~Hz}, 1 \mathrm{H}\right), 8.03$ (dd, $J=9.2,5.6 \mathrm{~Hz}, 1 \mathrm{H}$ ) $7.80-7.64(\mathrm{~m}, 2 \mathrm{H}), 7.47$ (dd, $J=8.5,3.0 \mathrm{~Hz}, 1 \mathrm{H}), 7.22(\mathrm{td}, J=8.6,3.0 \mathrm{~Hz}, 1 \mathrm{H}), 4.66(\mathrm{~d}, J=3.8 \mathrm{~Hz}$

$1 \mathrm{H}), 3.71-3.60(\mathrm{~m}, 1 \mathrm{H}), 3.53$ (tq, $J=7.6,3.7 \mathrm{~Hz}, 1 \mathrm{H}$ ): 3.16 (ddt, $J=11.2,7.3,4.5 \mathrm{~Hz}, 2 \mathrm{H}$ ), 2.74 (ddd,

$=11.8,8.5,3.4 \mathrm{~Hz}, 2 \mathrm{H}), 1.86-1.68(\mathrm{~m}, 2 \mathrm{H}), 1.46(\mathrm{dtd}, J=12.1,8.0,3.6 \mathrm{~Hz}, 2 \mathrm{H}), 1.15-1.05(\mathrm{~m}, 4 \mathrm{H})$.
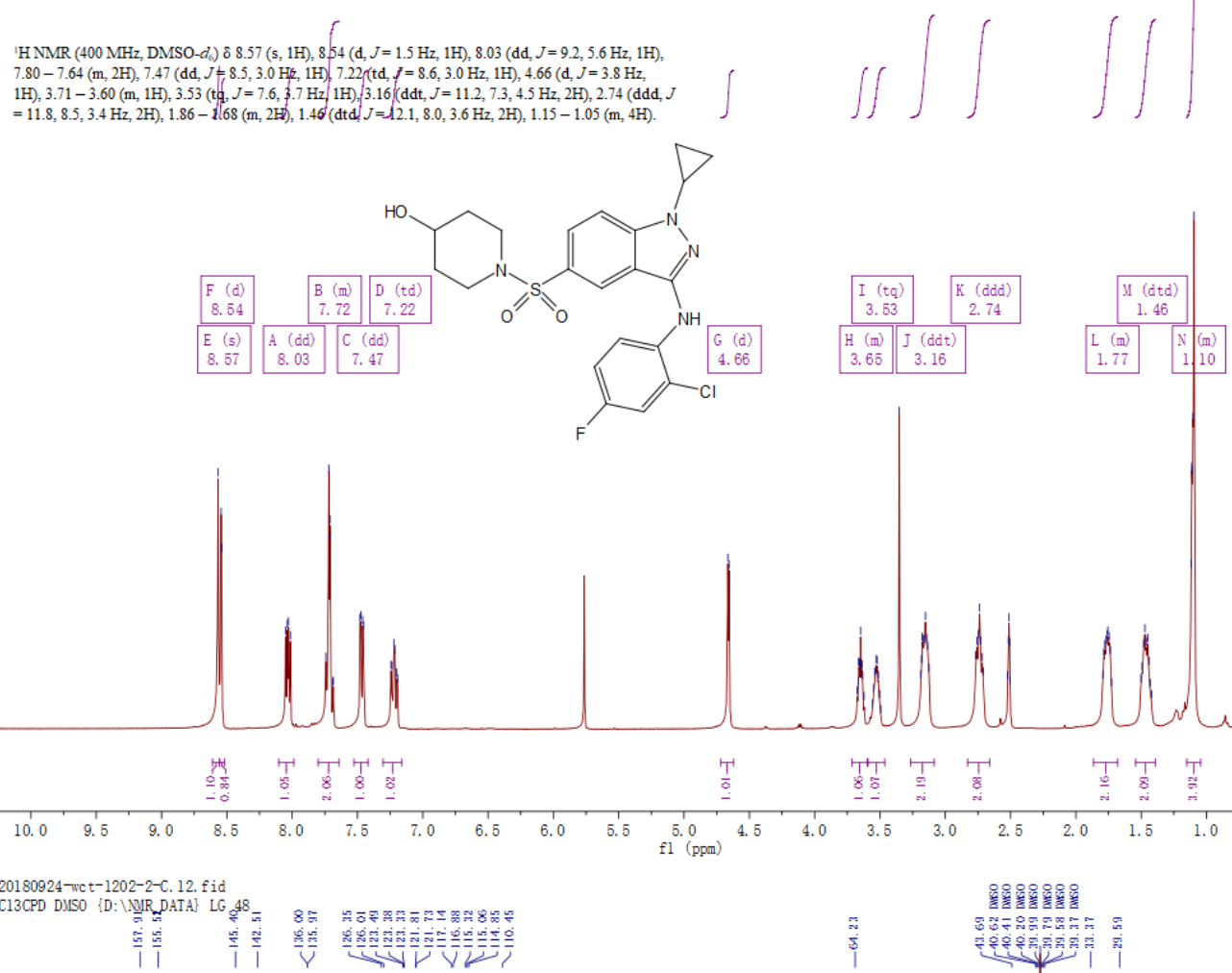

${ }^{13} \mathrm{CNMR}\left(101 \mathrm{MHz}, \mathrm{DMSO}-d_{\mathrm{s}}\right) \delta 156.71(\mathrm{~d}, J=240.9 \mathrm{~Hz}), 145.40,142.51,135.99(\mathrm{~d}, J=3.0 \mathrm{~Hz})$, $126.35,126.01,123.44(\mathrm{~d}, J=10.5 \mathrm{~Hz}), 123.33,121.77(\mathrm{~d}, J=8.2 \mathrm{~Hz}), 117.01(\mathrm{~d}, J=25.7 \mathrm{~Hz}), 115.32$

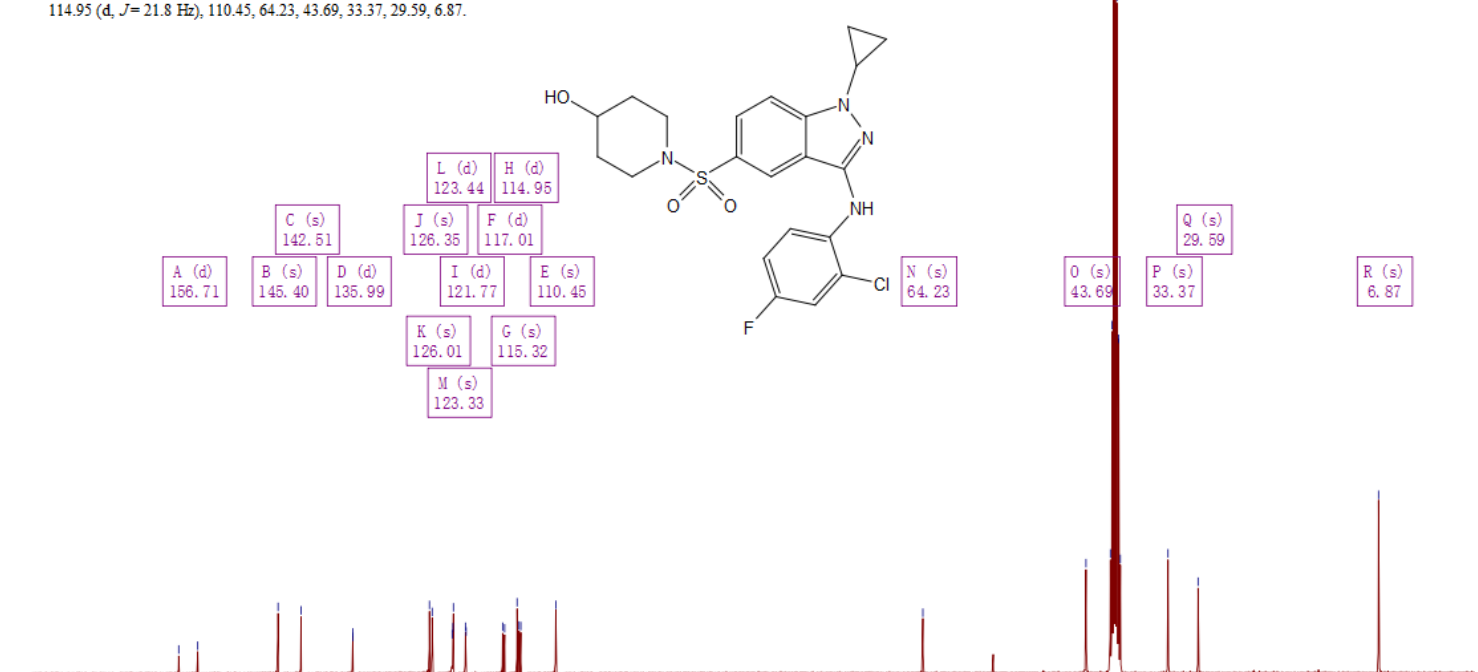

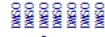
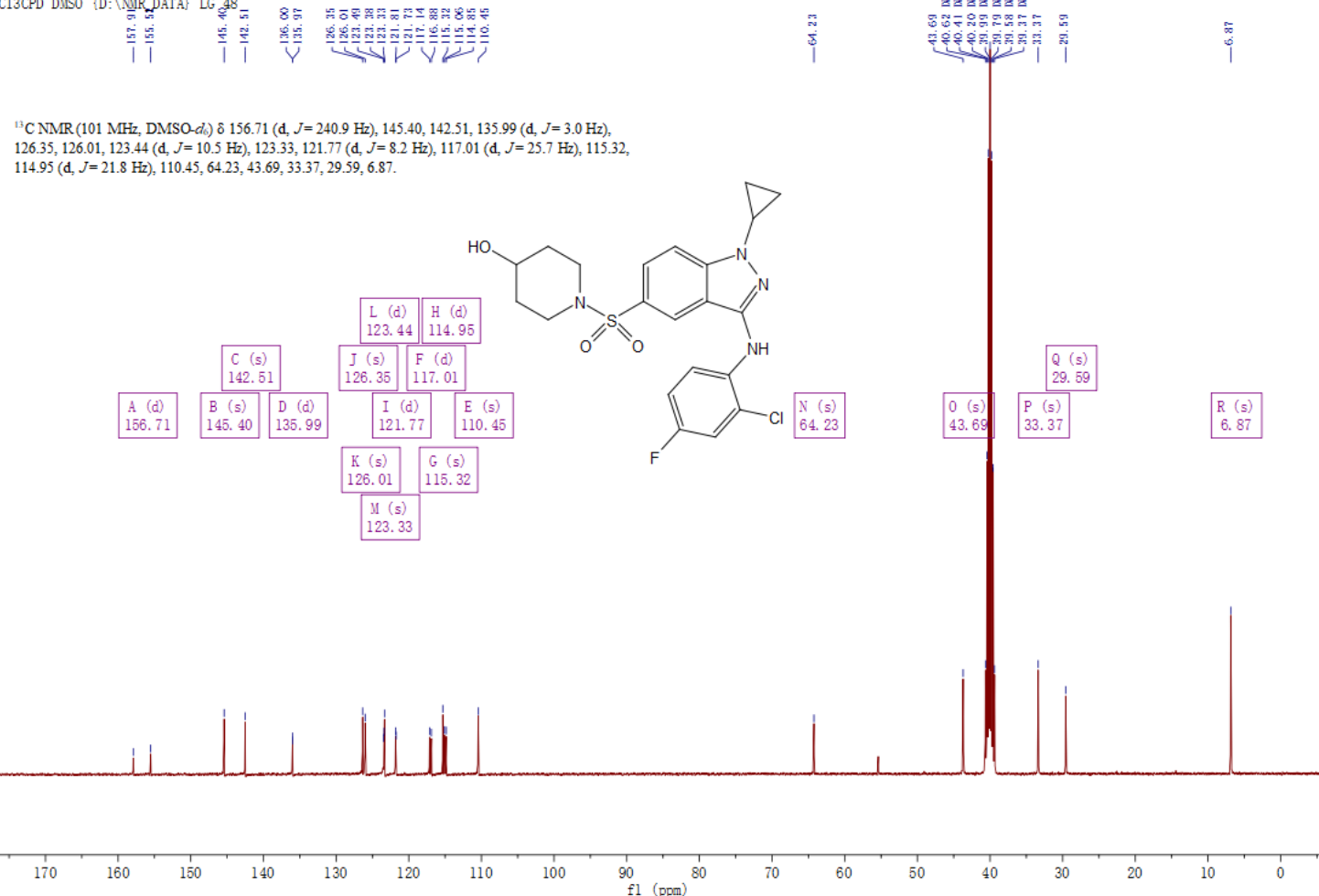


\section{Compound 19.}

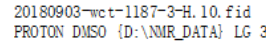

H NMR (400 MHz, DMSO- $\left.d_{\mathrm{s}}\right) \delta 9.63(\mathrm{~s}, 1 \mathrm{H}), 8.56(\mathrm{~d}, J=1.2 \mathrm{~Hz}, 1 \mathrm{f}), 7.96-7.82(\mathrm{~m}, 1 \mathrm{H}), 7.71(\mathrm{~s}$, $2 \mathrm{H}), 7.48-7.31(\mathrm{~m}, 2 \mathrm{H}), 4.66(\mathrm{~s}, 1 \mathrm{H}), 3.76-3.60(\mathrm{~m}, 1 \mathrm{H}), 3.50(\mathrm{~s}, 1 \mathrm{H}), 3.14(\mathrm{dt}, J=11.2,4.0 \mathrm{~Hz}, 2 \mathrm{H})$ 2.72 (ddd $J=11.7,8.4,3.4 \mathrm{~Hz}, 2 \mathrm{H}), 1.82-1.67(\mathrm{~m}, 2 \mathrm{H}), 1.44$ (dtd, $J=121.81,3.7 \mathrm{~Hz}, 2 \mathrm{H}), 1.18-$ $1.05(\mathrm{~m}, 4 \mathrm{H})$
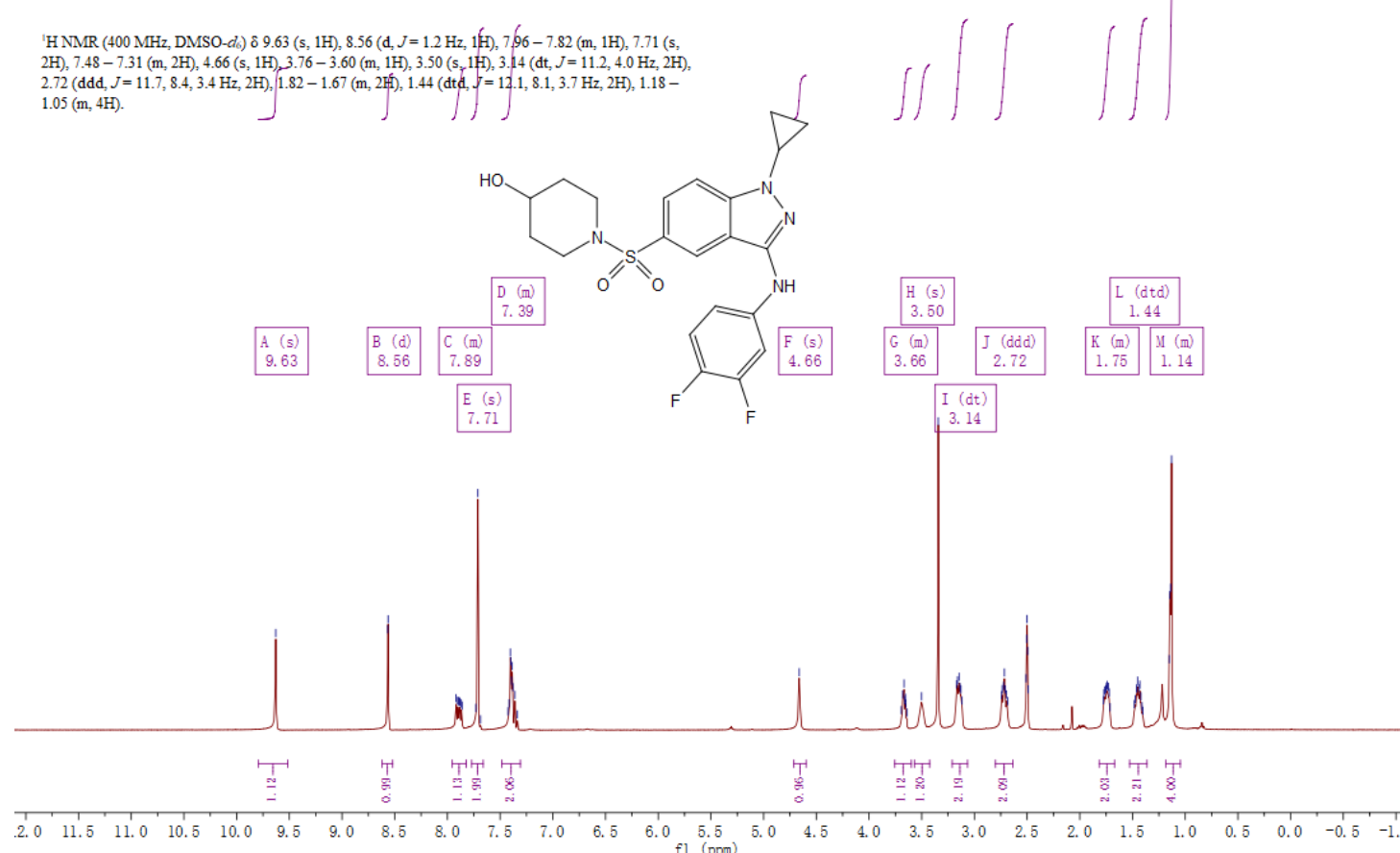

20180907-wct-1187-3-C. 10. fid

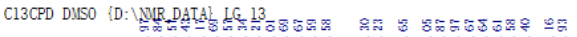

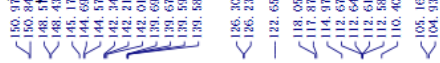

${ }^{13} \mathrm{C} \mathrm{NMR}\left(101 \mathrm{MHz}\right.$, DMSO- $\left.d_{\mathrm{l}}\right) \delta 151.40-146.76(\mathrm{~m}), 145.17,143.45$ (dd, $\left.J=237.0,12.4 \mathrm{~Hz}\right), 142.01$,

$139.63(\mathrm{dd} J=9,7,2.2 \mathrm{~Hz}), 126.30,12623,122.65,117.96(\mathrm{~d}, J=17.7 \mathrm{~Hz}), 114.97,112.62(\mathrm{dd}, J=5.3$

$2.8 \mathrm{~Hz}), 110.40,105.05(\mathrm{~d}, J=223 \mathrm{~Hz}), 6422,43.71,33,36,29.62,686$.
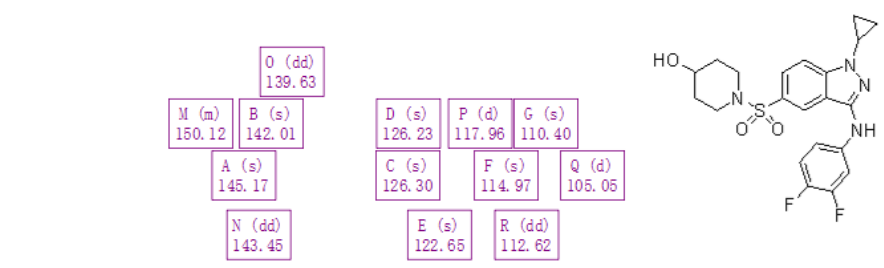

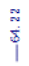

\begin{tabular}{|l|}
\hline$H(s)$ \\
64.22 \\
\hline
\end{tabular}
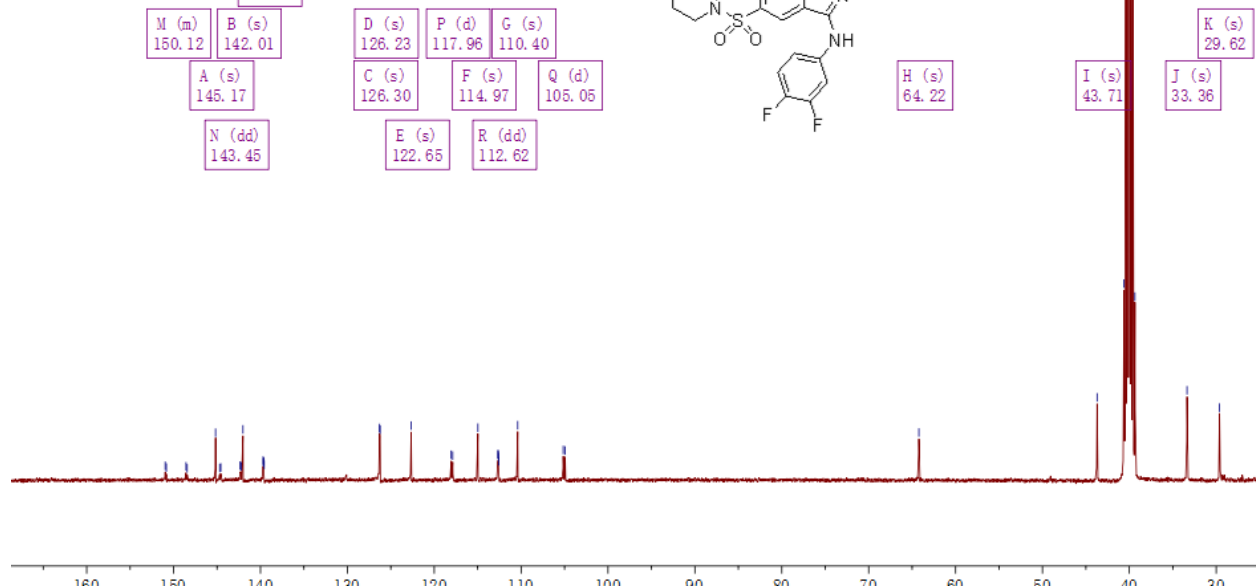

${ }_{\mathrm{f} 1}^{10}(\mathrm{pmm})$ 


\section{Compound 20.}

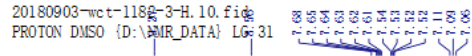

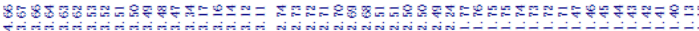

ن

H NMR ( $\left.400 \mathrm{MHz}, \mathrm{DMSO}-d_{\mathrm{s}}\right) \delta 9.27(\mathrm{~s}, 1 \mathrm{H}), 8.56(\mathrm{~s}, 1 \mathrm{H}), 7.68(\mathrm{~s}, 2 \mathrm{H}), 7.63$ (dt, $J=8.2,3.6 \mathrm{~Hz}, 1 \mathrm{H}$ ),

$7.53(\mathrm{dd}, J=6.9,2.8 \mathrm{~Hz}, 1 \mathrm{H}), 7.09(\mathrm{t}, J=9.2 \mathrm{~Hz}, 1 \mathrm{H}), 4.66(\mathrm{~s}, 1 \mathrm{H}), 3.64(\mathrm{p}, J=5.3 \mathrm{~Hz}, 1 \mathrm{H}), 3.50$ (tt, $J=$

$7.9,3.6 \mathrm{~Hz}, 1 \mathrm{H}$ ), $3.22-3.07(\mathrm{~m}, 2 \mathrm{H}), 2.71$ (ddd, $J=11.9,8.6,3.5 \mathrm{~Hz}, 2 \mathrm{H}), 2.24(\mathrm{~s}, 3 \mathrm{H}$ ), 1.74 (ddd, $J=$

$13.9,7.3,3.6 \mathrm{~Hz}, 2 \mathrm{H}), 1.44$ (dtd, $J=12.3,8.2,3.6 \mathrm{~Hz}, 2 \mathrm{H}), 1.12(\mathrm{~d}, J=5.2 \mathrm{~Hz}, 4 \mathrm{H})$.

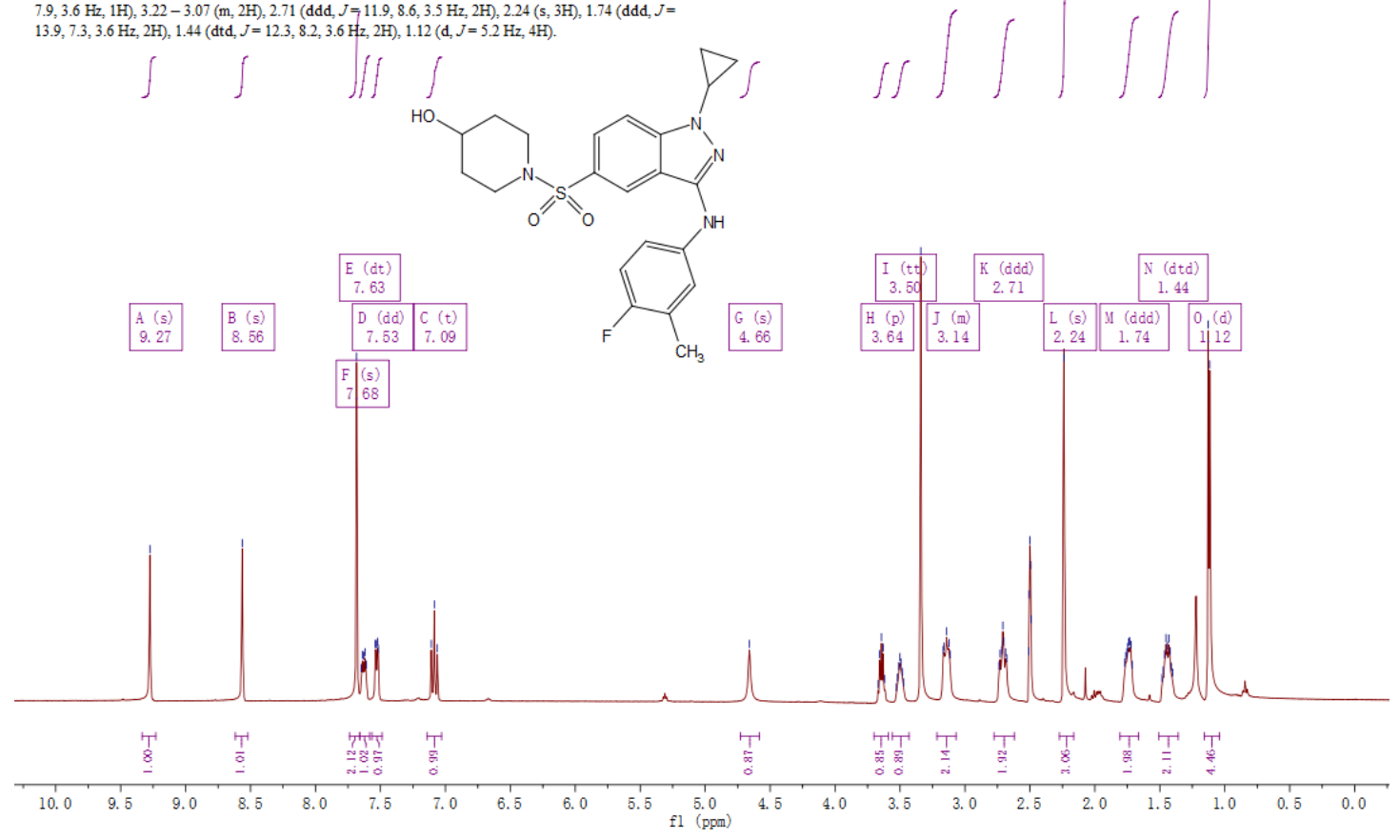

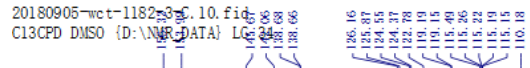

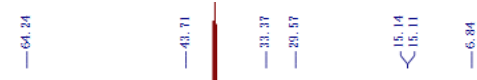

C NMR (101 MHz, DMSO-d $)^{2} \delta 155.16(\mathrm{~d}, J=234.8 \mathrm{~Hz}), 145.67,142.06,138.67(\mathrm{~d}, J=2.2 \mathrm{~Hz})$

$126.01(\mathrm{~d}, J=29.0 \mathrm{~Hz}), 124.46(\mathrm{~d}, J=18.0 \mathrm{~Hz}), 122.78,119.17(\mathrm{~d}, J=4.0 \mathrm{~Hz}), 115.49,115.24(\mathrm{~d}, J=4.1$

$\mathrm{Hz}), 115.19,115.15,110.18,64.24,43.71,33.37,29.57,15.13(\mathrm{~d}, J=2.9 \mathrm{~Hz}), 6.84$.

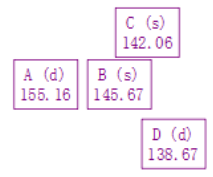

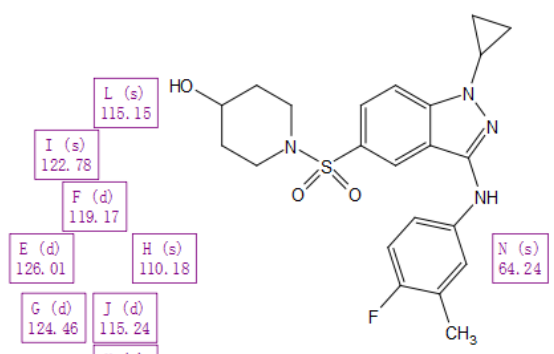

K (s)

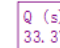

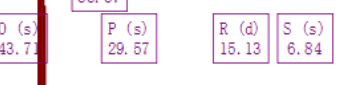

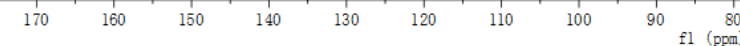




\section{Compound 21.}
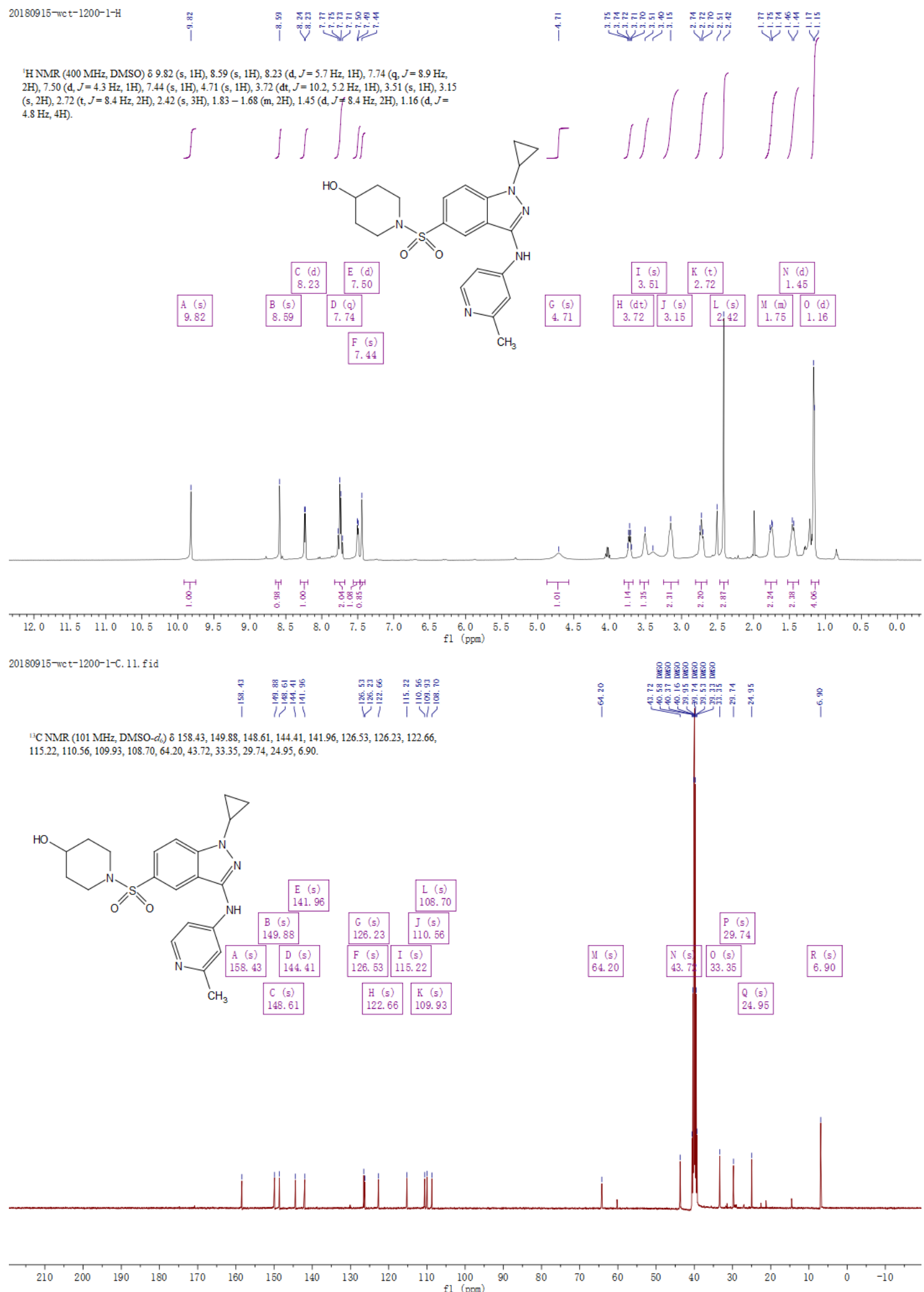


\section{Compound 22.}

20181029-wct-1228-1-H. 10, fid
PROTON DUSO \{D: INIR_DATA\} LG 36

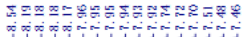

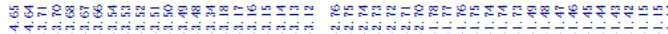

H NMR (400 MHz, DMSO-d s $^{2} 9.75(\mathrm{~s}, 1 \mathrm{H}), 8.54(\mathrm{~s}, 1 \mathrm{H}), 8.18$ (dd, $J=5.5,2.9 \mathrm{~Hz}, 1 \mathrm{H}$ ), $8.02-7.87$ $(\mathrm{m}, 1 \mathrm{H}), 7.72(\mathrm{~s}, 2 \mathrm{H}), 7.48(\mathrm{t}, J=9.1 \mathrm{~Hz}, 1 \mathrm{H}), 4.64(\mathrm{~d}, J=3.8 \mathrm{~Hz}, 1 \mathrm{H}), 3.68(\mathrm{p}, J=5.4 \mathrm{~Hz}, 1 \mathrm{H}), 3.51$ (tt

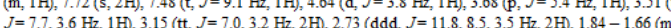
$2 \mathrm{H}), 1.45(\mathrm{dtd}, J=12.3,8.2,3.7 \mathrm{~Hz}, 2 \mathrm{H}), 1.15(\mathrm{~d}, J=6.4 \mathrm{~Hz}, 4 \mathrm{H}$.

$$
\int
$$

$\iiint \int \mathrm{H}$<smiles>C1=CC2CCCC2C1</smiles><smiles>C=CCC=CCCC</smiles><smiles>c1ccccc1</smiles>

A ( $(s)$
9.75

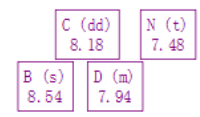

\begin{tabular}{c|cc}
$B(s)$ & D (II) \\
8.54 & 7.94 \\
\hline
\end{tabular}

$\begin{array}{r}F(d) \\ 4.64 \\ \hline\end{array}$
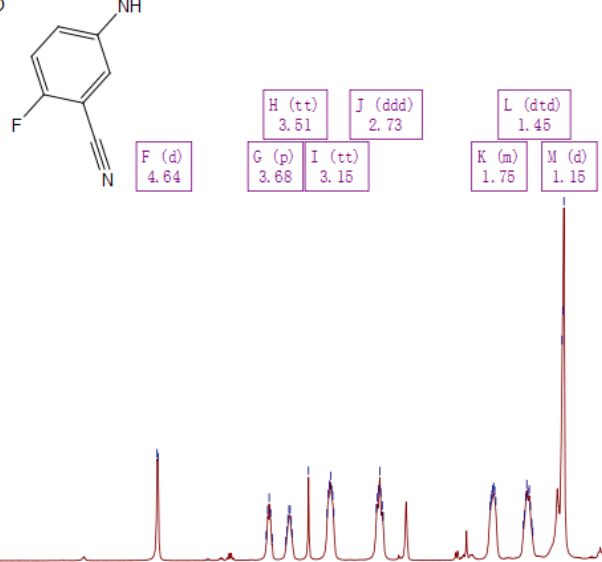

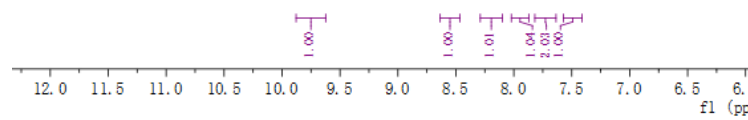

20181030-wct-1228-1-C. 10. fid
C13CPD DUSO [D: $\backslash$ NNR_DATA] LG 2]

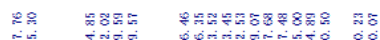
$\frac{1}{8}$

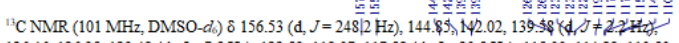
$126.46,126.35,123.49(\mathrm{~d}, J=7.5 \mathrm{~Hz}), 122.53,119.07,117.58(\mathrm{~d}, J=20.5 \mathrm{~Hz}), 115.00,114.89,110.50$ $100.15(\mathrm{~d}, J=16.0 \mathrm{~Hz}), 64.22,43.69,33.37,29.67,6.87$.

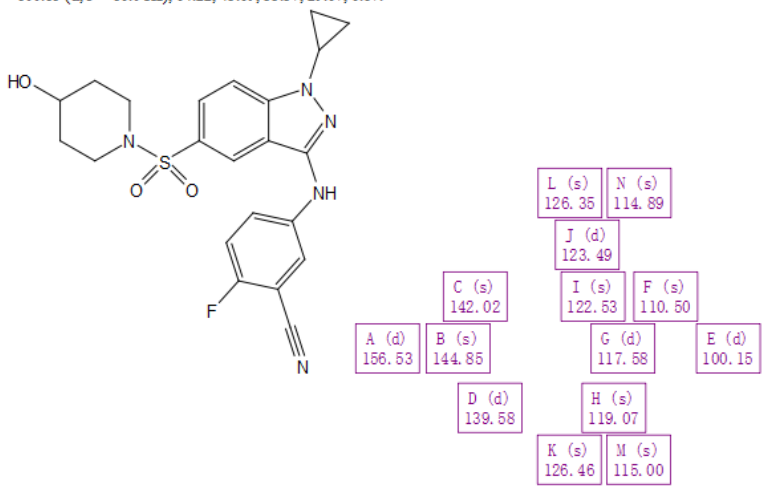

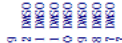

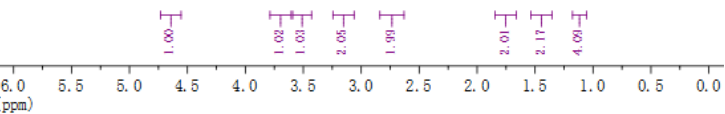




\section{Compound 23.}

$20181105-$ - $\mathrm{cot}-1210-1-\mathrm{H}$. 10. fid $\mathrm{de}$ PROTON DMSO [D: LNIR_DATA\} LG

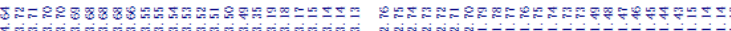

'H NMR ( $400 \mathrm{MHz}$, DMSO-d $\left.d_{s}\right) \delta 10.19(\mathrm{~s}, 1 \mathrm{H}), 8.71(\mathrm{~d}, J=1.2 \mathrm{~Hz}, 1 \mathrm{H}), 8.23$ (d, $\left.J=3.0 \mathrm{~Hz}, 1 \mathrm{H}\right), 8.05$

(dd, $J=9.2,3.9 \mathrm{~Hz}, 1 \mathrm{H}), 7.78-7.66(\mathrm{~m}, 3 \mathrm{H}), 4.65$ (d, $J=3.8 \mathrm{~Hz}, 1 \mathrm{H}), 3.69$ (tt, $J=6.6,4.5 \mathrm{~Hz}, 1 \mathrm{H}), 3.52$

(tq, $J=7.4,3.6 \mathrm{~Hz}, 1 \mathrm{H}), 3.18-3.10(\mathrm{~m}, 2 \mathrm{H}), 2.73$ (ddd, $J=11.8,8.4,3.4 \mathrm{~Hz}, 2 \mathrm{H}), 1.76$ (ddt, $J=14.0$,

$7.2,3.5 \mathrm{~Hz}, 2 \mathrm{H}$ ), 1.46 (dtd, $J=11.9,7.9,3.6 \mathrm{~Hz}, 2 \mathrm{H}), 1.19-1.07(\mathrm{~m}, 4 \mathrm{H})$.
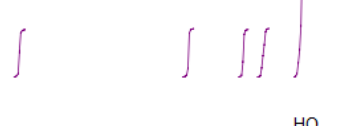

$\int 1$

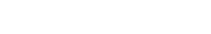

\begin{tabular}{|c|c|}
\hline & \begin{tabular}{|l|} 
C (d) \\
8.23 \\
\end{tabular} \\
\hline $\begin{array}{l}\text { B (d) } \\
8.71\end{array}$ & \begin{tabular}{|l} 
D (d \\
8.0
\end{tabular} \\
\hline
\end{tabular}<smiles>OC1CCNCC1</smiles>

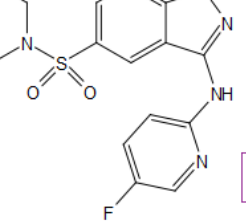

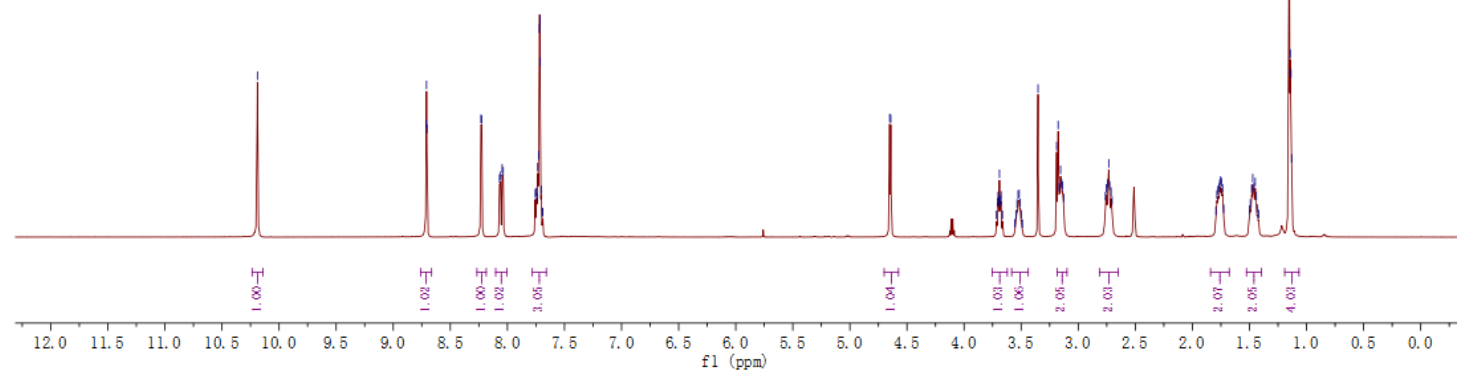

20181108-wct-1210-1-C. 10. fid

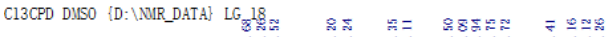

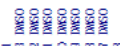

${ }^{13} \mathrm{C} \mathrm{NMR}\left(101 \mathrm{MHz}, \mathrm{DMSO}-d_{d}\right)$ )

$\mathrm{Hz}), 126.50,126.09,125.85(\mathrm{~d}, J=19.9 \mathrm{~Hz}), 123.72,115.41,111.14(\mathrm{~d}, J=4.0 \mathrm{~Hz}), 110.26,64.26,43.71$,

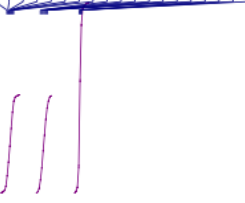
$33.38,29.64,6.84$
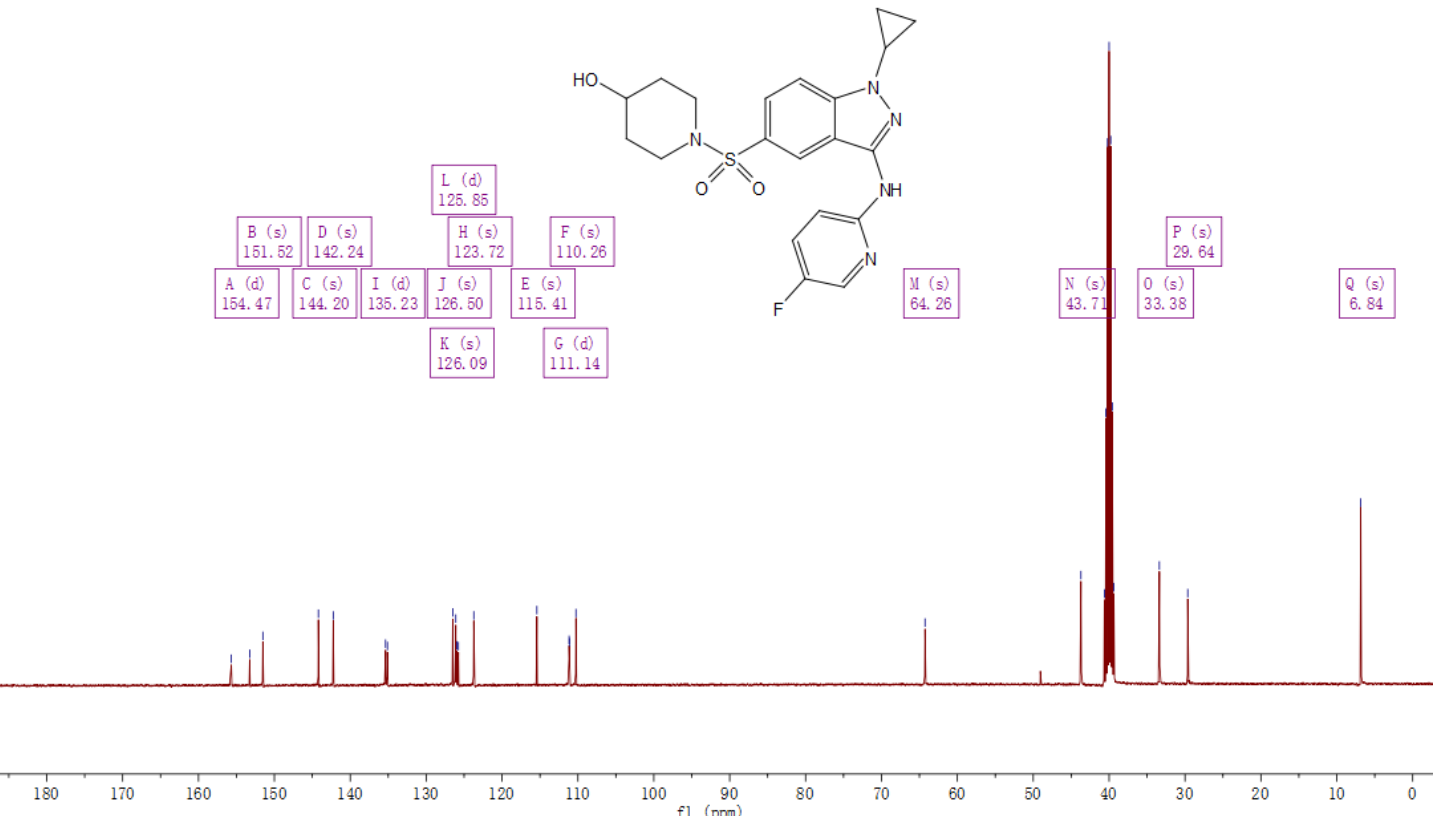


\section{Compound 24.}

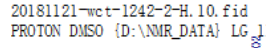

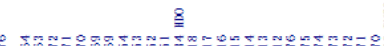

曼

'H NMR (400 MHz, DMSO- $\left.d_{s}\right) \delta 10.02(\mathrm{~s}, 1 \mathrm{H}), 8.66(\mathrm{~s}, 1 \mathrm{H}), 8.10(\mathrm{~s}, 1 \mathrm{H}), 7.86(\mathrm{~d}, J=5.6 \mathrm{~Hz}, 1 \mathrm{H}), 7.78$

$-7.66(\mathrm{~m}, 2 \mathrm{H}), 4.64(\mathrm{~d}, J=3.8 \mathrm{~Hz}, 1 \mathrm{H}), 3.71(\mathrm{td}, J=6.3,3.2 \mathrm{~Hz}, 1 \mathrm{H}), 3.52(\mathrm{tq}, J=7.6,3.7 \mathrm{~Hz}, 1 \mathrm{H}), 3.16$

(ddt, $J=11.2,7.2,4.2 \mathrm{~Hz}, 2 \mathrm{H}$ ), 2.73 (ddd, $J=11.7,8.2,3.3 \mathrm{~Hz}, 2 \mathrm{H}$ ), 2.31 (s, $3 \mathrm{H}), 1.76$ (ddt, $J=14.0$,

$7.2,3.5 \mathrm{~Hz}, 2 \mathrm{H}$ ), 1.46 (dtd, $J=12.1,8.1,3.6 \mathrm{~Hz}, 2 \mathrm{H}), 1.19-1.07$ (m, $4 \mathrm{H}$ ).

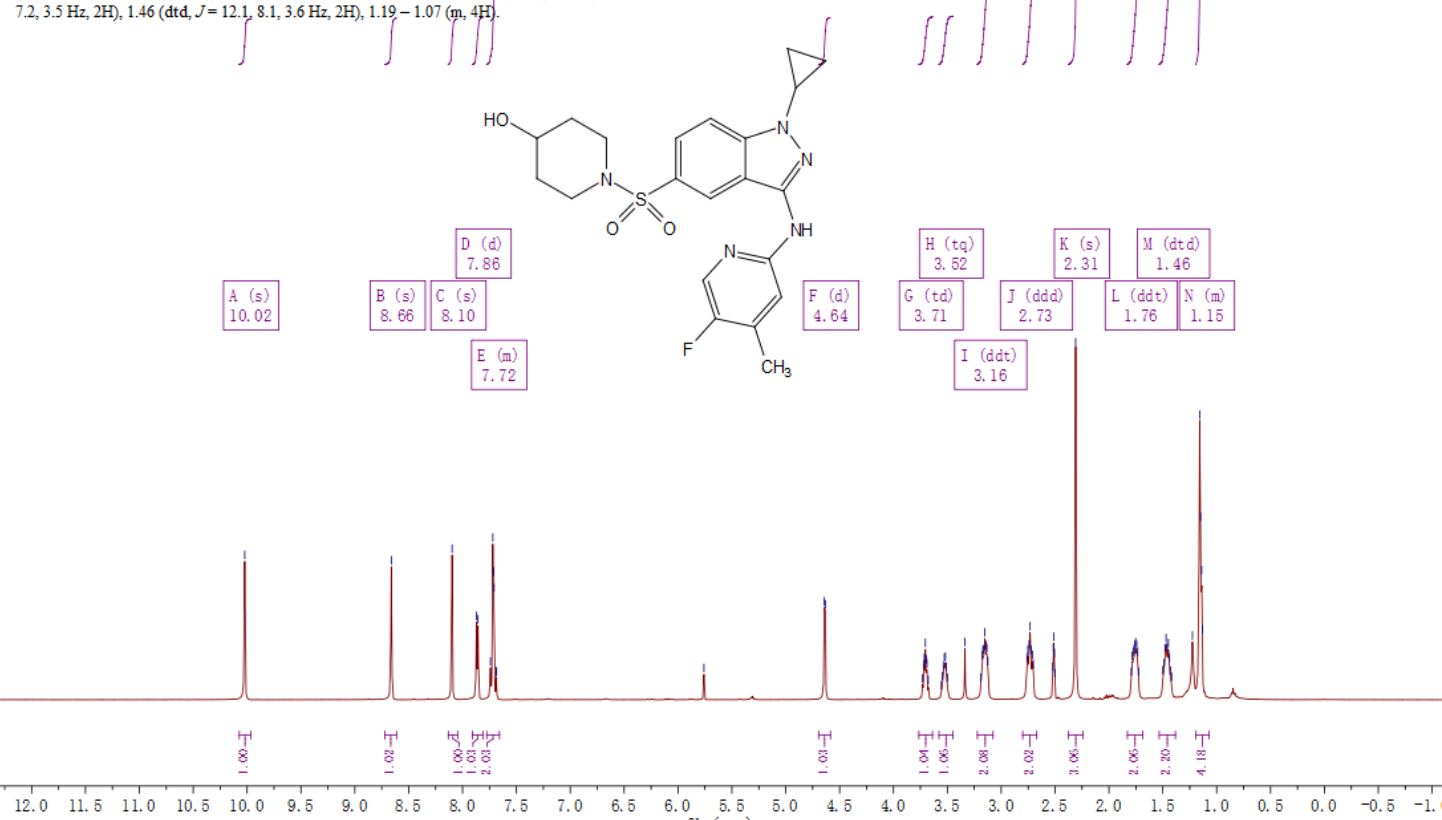

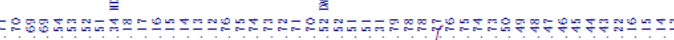

C13CPD DMSO \{D: LNIR_DATA $L$ LS 16

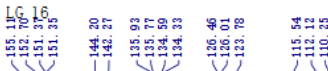

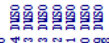

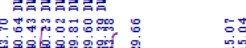

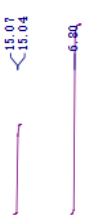

${ }^{13} \mathrm{C} \mathrm{NMR}\left(101 \mathrm{MHz}, \mathrm{DMSO}-d_{0}\right) 6133.91(\mathrm{~d}, J=2421 \mathrm{~Hz}), 151.36(\mathrm{~d}, J=1.9 \mathrm{~Hz}), 144.20,142.27$ $135.85(\mathrm{~d}, J=16.1 \mathrm{~Hz}), 134.46(\mathrm{~d}, J=25.7 \mathrm{~Hz})$,
$43.70,33.38,29.66,15.05(\mathrm{~d}, J=2.8 \mathrm{~Hz}), 6.80$.

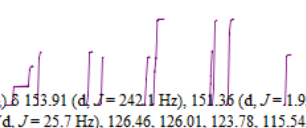

$\iint_{\mathrm{Hz}), 14.20,142.27,26}$
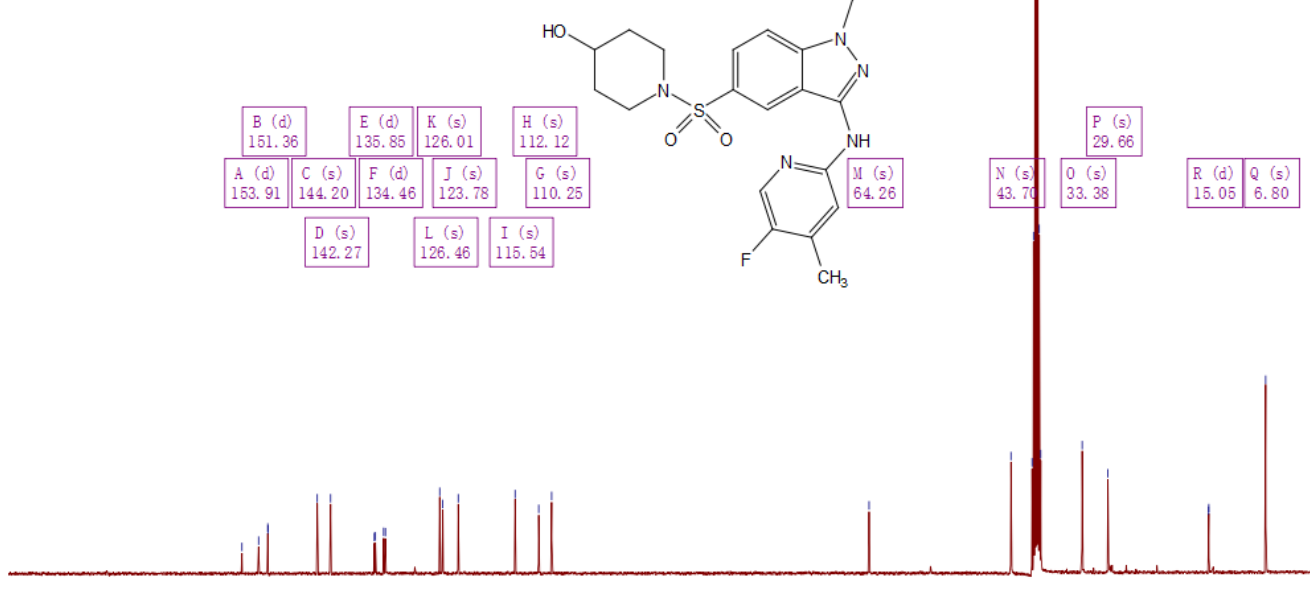

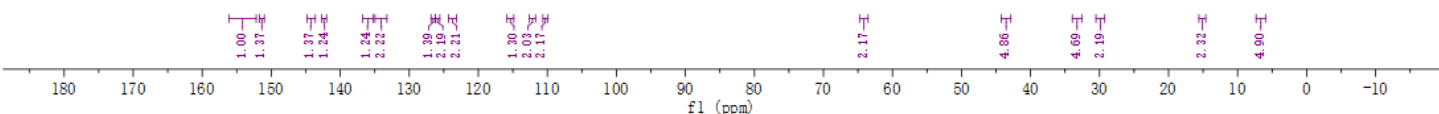




\section{Compound 25.}

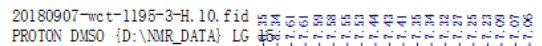

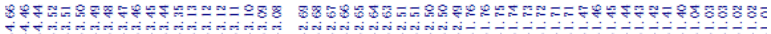

'H NMR (400 MHz, DMSO- $\left.d_{6}\right) 88.34(\mathrm{~d}, J=1.6 \mathrm{~Hz}, 1 \mathrm{H}), 7.65-7.50(\mathrm{~m}, 2 \mathrm{H}), 7.42(\mathrm{~d}, J=7.2 \mathrm{~Hz}, 2 \mathrm{H})$, $7.34(\mathrm{t}, J=7.4 \mathrm{~Hz}, 2 \mathrm{H}), 7.25(\mathrm{t}, J=7.3 \mathrm{~Hz}, 1 \mathrm{H}), 7.07(\mathrm{t}, J=5.8 \mathrm{~Hz}, 1 \mathrm{H}), 4.66(\mathrm{~s}, 1 \mathrm{H}), 4.45(\mathrm{~d}, J=5.8$ $\mathrm{Hz}, 2 \mathrm{H}), 3.48$ (qt, $J=6.8,3.8 \mathrm{~Hz}, 2 \mathrm{H}), 3.11$ (ddd, $J$ (dt $1.2,6.9,3.7 \mathrm{~Hz}, 2 \mathrm{H}$ ), 2.66 (ddd, $J=11.8,8.5,3.4$
$\mathrm{~Hz}, 2 \mathrm{H}), 1.73$ (ddt, $J=13.9,7.2,3.5 \mathrm{~Hz}, 2 \mathrm{H}), 1.43$ (dtd. $J=12.2,8.2,3.7 \mathrm{~Hz}, 2 \mathrm{H}), 1.03$ (dt, $J=8.3,2.8$ $\mathrm{Hz}, 4 \mathrm{H})$.
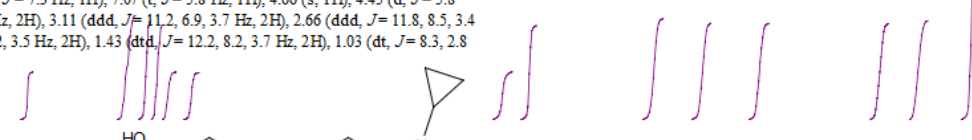

\begin{tabular}{|l|r|r|}
\hline $\begin{array}{l}\text { A (d) } \\
8.34\end{array}$ & $\begin{aligned} \mathrm{F}(\mathrm{m}) \\
7.57\end{aligned}$ & $\begin{array}{l}\text { B (t) } \\
7.07\end{array}$ \\
\hline
\end{tabular}

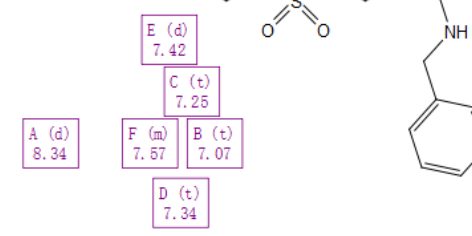<smiles>C1=N[SH]2(=CC2)OC1</smiles><smiles>CCc1ccccc1C1CC2CCC1[C@H]2C</smiles>
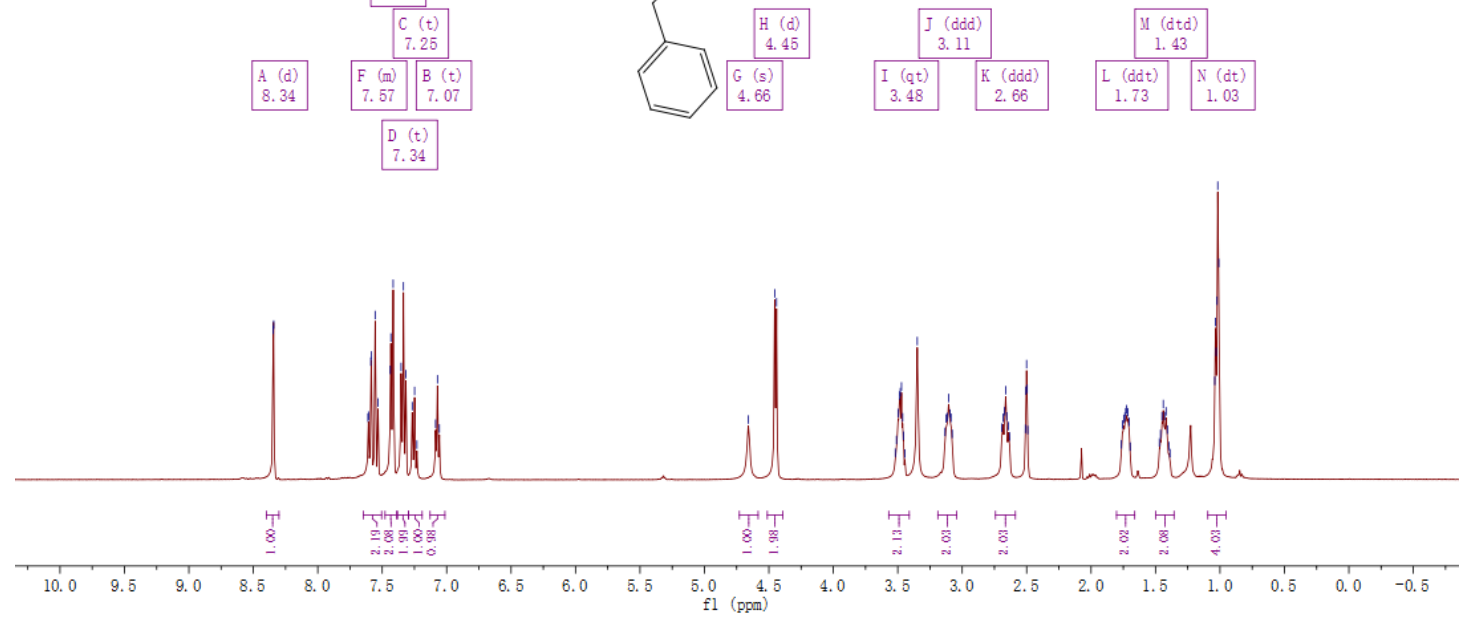

20180909-wct-1195-3-C. 10, fid
C13CPD DUSO \{D: $\backslash$ NIR_DATA\} LG 5

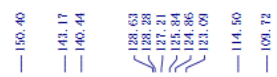

${ }^{12} \mathrm{CNMR}\left(101 \mathrm{MHz}\right.$, DMSO- $\left.d_{6}\right) \delta 150.40,143.17,140.44,128.63,128.28,127.21,125.84,124.86$ $123.09,114.50,109.72,64.28,47.04,43.70,33.37,29.23$
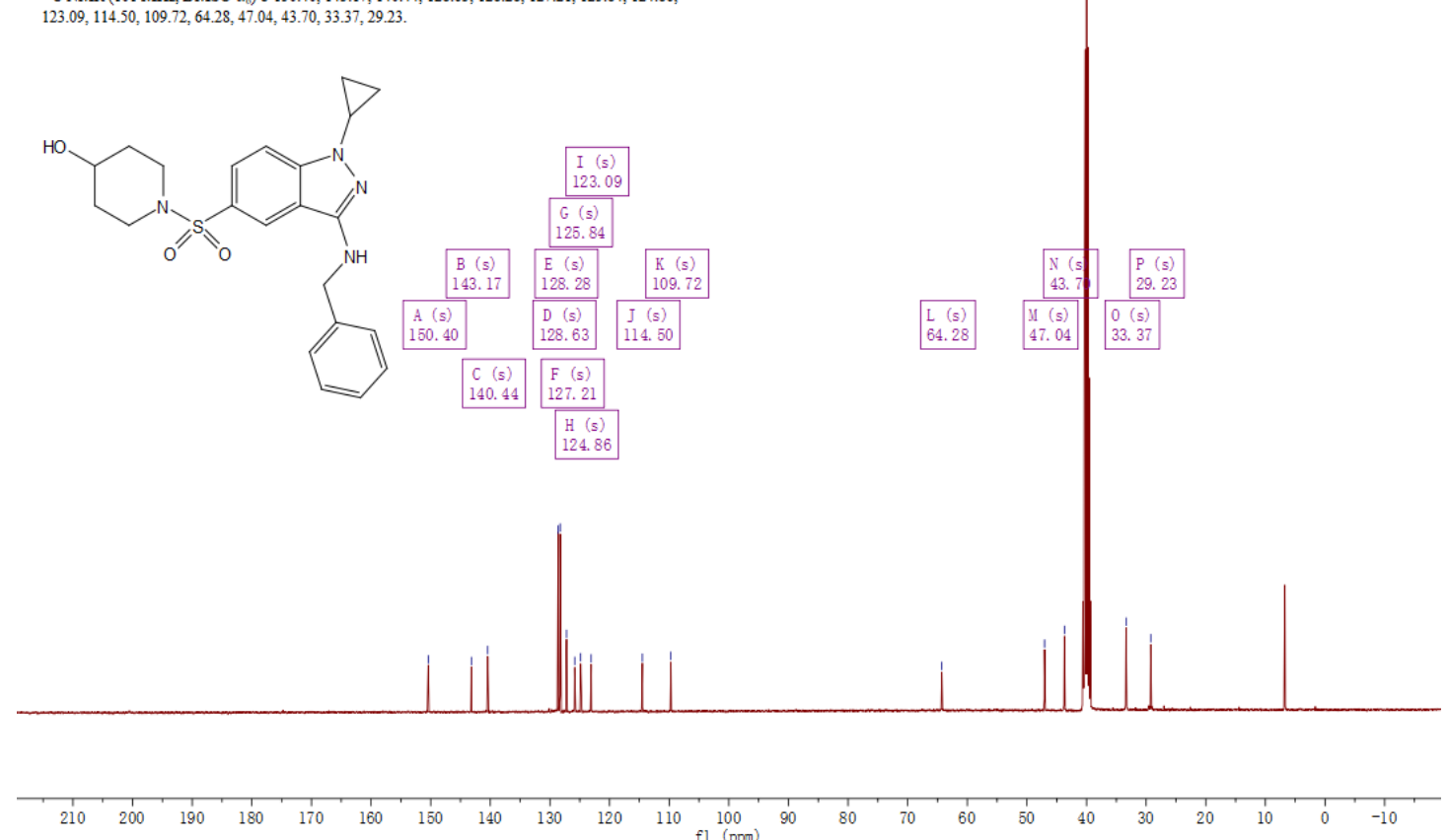


\section{Compound 26.}

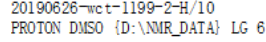
نivicing

'H NMR (400 MHz, DMSO-d $d_{\text {) }} \delta 8.31(\mathrm{~s}, 1 \mathrm{H}), 7.64-7.57(\mathrm{~m}, 1 \mathrm{H}), 7.55(\mathrm{~d}, J=8.9 \mathrm{~Hz}, 1 \mathrm{H}), 7.45$ (dd, $J$ $=8.4,5.6 \mathrm{~Hz}, 2 \mathrm{H}), 7.16(\mathrm{t}, J=8.7 \mathrm{~Hz}, 2 \mathrm{H}, 7.07(\mathrm{t}, J=5.9 \mathrm{~Hz}, 1 \mathrm{H}), 4.64(\mathrm{~d}, J=3.8 \mathrm{~Hz}, 1 \mathrm{H}), 4.42(\mathrm{~d}, J=$
$5.7 \mathrm{~Hz}, 2 \mathrm{H}), 3.61-3.42(\mathrm{~m}, 2 \mathrm{H}), 3.10(\mathrm{t}, J=8.8 \mathrm{~Hz}, 2 \mathrm{H}), 2.75-2.59(\mathrm{~m}, 2 \mathrm{H}), 1.84-1.64(\mathrm{~m}, 2 \mathrm{H}), 1.53$ $-1.32(\mathrm{~m}, 2 \mathrm{H}), 1.02(\mathrm{t}, J=6.4 \mathrm{~Hz}, 4 \mathrm{H})$.

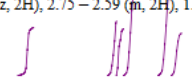<smiles>O=S(=O)(c1ccc2c(c1)c(NCc1ccc(F)cc1)nn2C1CC1)N1CCC(O)CC1</smiles>
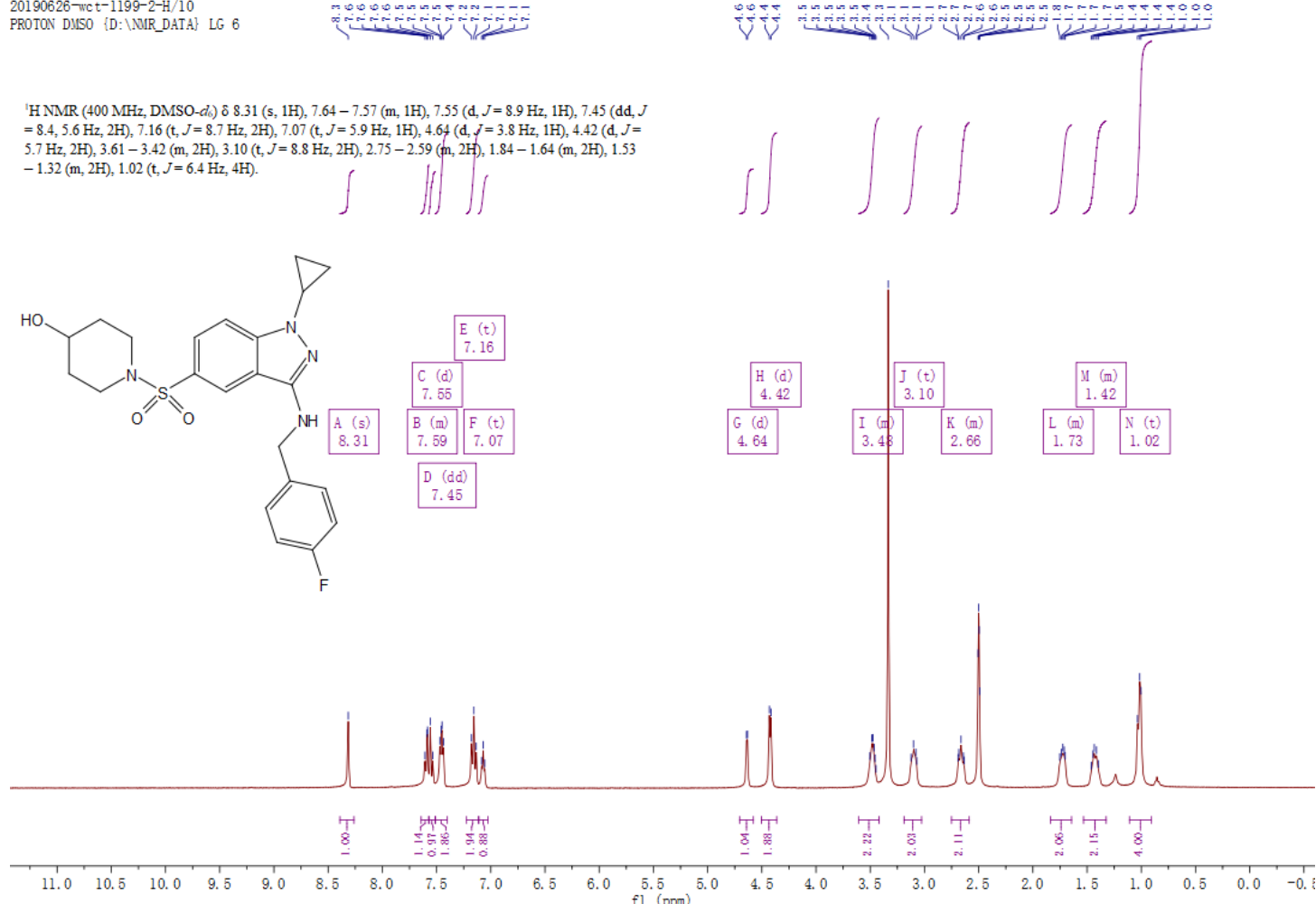

$20190627-w c t-1199-2-\mathrm{C} / 10$

C13CPD DUSO \{D: $\backslash$ NUR_DATA\} LG 22

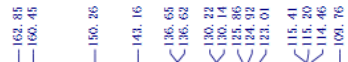

"CNMR (101 MHz, DMSO-d $\delta$ 161.65 (d $J=2420 \mathrm{~Hz}), 150.26,143.16,136.63(\mathrm{~d}, J=2.9 \mathrm{~Hz})$ $130.18(\mathrm{~d}, J=8.0 \mathrm{~Hz}), 125.86,124.92,123.01,115.31(\mathrm{~d}, J=21.2 \mathrm{~Hz}), 114.46,109.76,64.27,46.26$ $43.19,33,37,29.23,6.73$

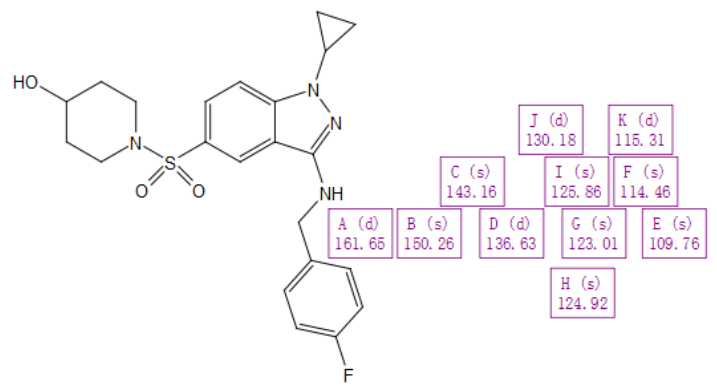

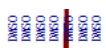

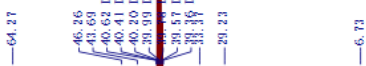

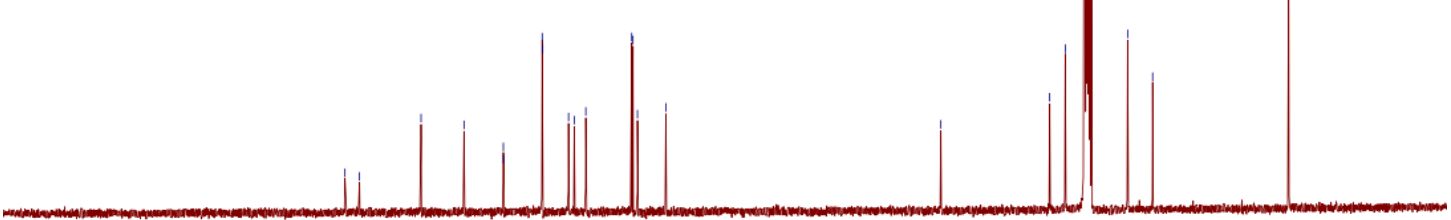

$\begin{array}{llllllllllll}210 & 200 & 190 & 180 & 170 & 160 & 150 & 140 & 130 & 120 & 110 & 100\end{array}$ 


\section{Compound 27.}

20190117-wct-1292-1-H. 10, fid
PROTON DUSO \{D: $\backslash$ NMR_DATA\} LG 49

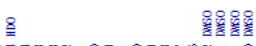

Fo

'H NMR (400 MHz, DMSO-d $d_{\text {s) }} 88.34$ (d, $\left.J=1.6 \mathrm{~Hz}, 1 \mathrm{H}\right), 7.58$ (dd, $\left.J=8.8,1.7 \mathrm{~Hz}, 1 \mathrm{H}\right), 7.51$ (d, $J=$

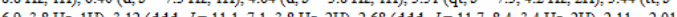

(m, 2H) $1.80-170$ (m,

$1.22(\mathrm{~m}, 5 \mathrm{H}), 1.09-0.98(\mathrm{~m}, 4 \mathrm{H})$
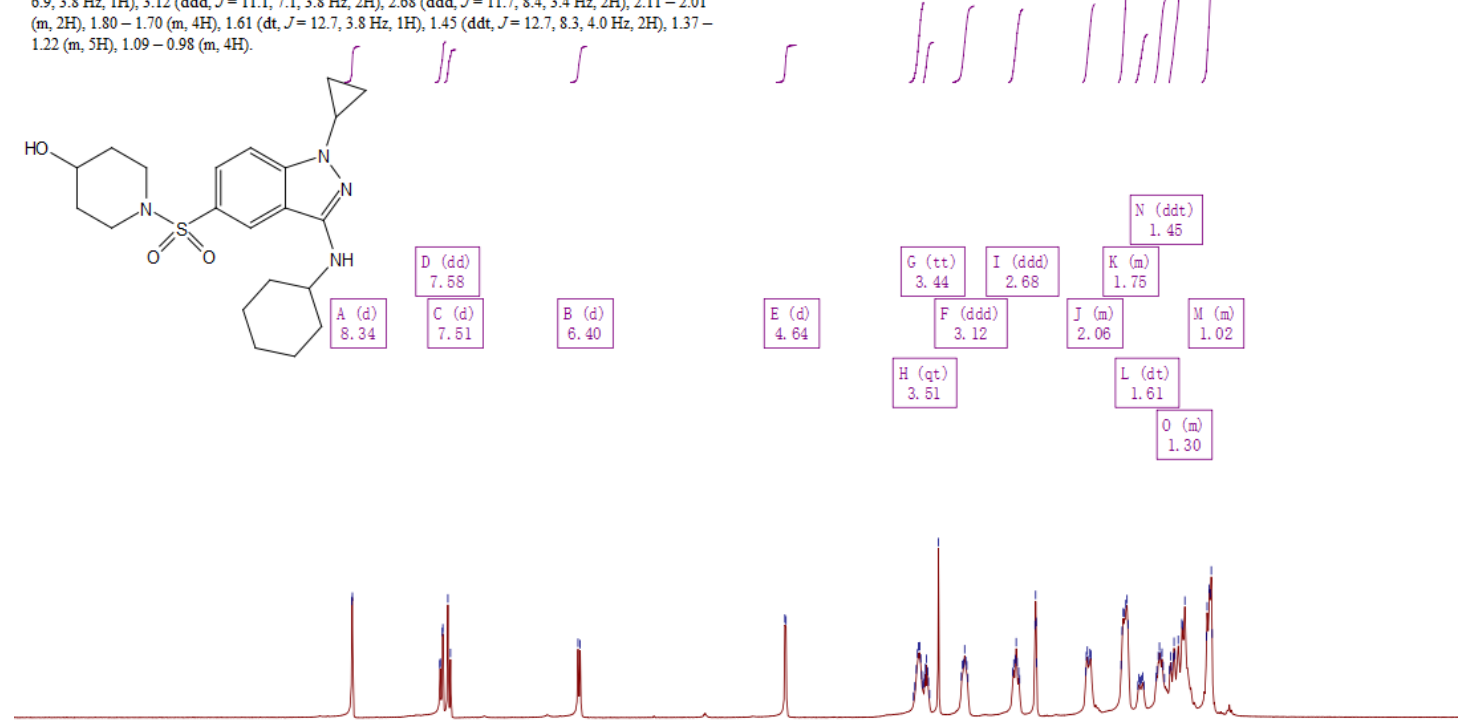

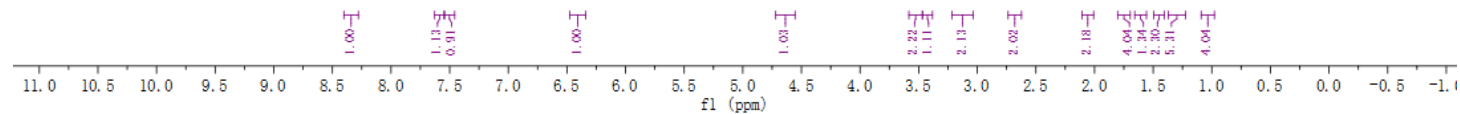

20190117-wct-1292-2-C. 10, fid
C13CPD DUSO $\{$ D: $\backslash$ NIR_DATA LG 27

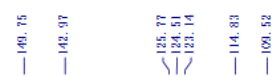

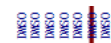

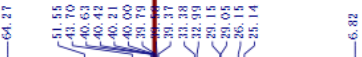

${ }^{13} \mathrm{C}$ NMR $\left(101 \mathrm{MHz}, \mathrm{DMSO}-d_{6}\right) \delta 149.75,142.97,125.77,124.51,123.14,114.83,109.52,64.27,51.55$ $43.70,33.38,32.99,29.15,26.15,25.14,6.82$<smiles>O=S(=O)(c1ccc2c(c1)c(NC1CCCCC1)nn2C1CC1)N1CCC(O)CC1</smiles>

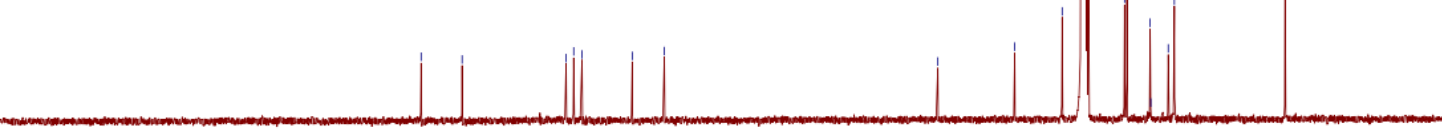

$\begin{array}{llllllllllll}210 & 200 & 190 & 180 & 170 & 160 & 150 & 140 & 130 & 120 & 110 & 100\end{array}$ 


\section{Compound 28.}

20190122-wct-1299-1-H. 10, fid
PROTON DUSO [D: $\backslash$ NMR_DATA\} LG 46 $\stackrel{8}{\underline{x}}$ 糬署罯

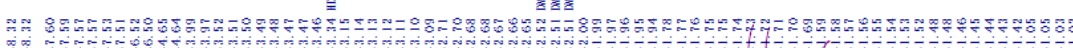
H NMR ( $400 \mathrm{MHz}$, DMSO- $\left.d_{\text {e }}\right) \delta 8.32$ (d, $J=1.5 \mathrm{~Hz}, 1 \mathrm{H}$ ), 7.58 (dd, $J=8.9,1.7 \mathrm{~Hz}, 1 \mathrm{H}$ ), 7.52 (d, $J=$ $8.8 \mathrm{~Hz}, 1 \mathrm{H}), 6.51(\mathrm{~d}, J=6.4 \mathrm{~Hz}, 1 \mathrm{H}), 4.64(\mathrm{~d}, J=3.6 \mathrm{~Hz}, 1 \mathrm{H}), 3.97(\mathrm{p}, J=6.3 \mathrm{~Hz}, 1 \mathrm{H}), 3.49$ (dtt, $J=$ $16.8,6.8,3.8 \mathrm{~Hz}, 2 \mathrm{H}), 3.12$ (ddd, $J=11.1,7.1,3.7 \mathrm{~Hz}, 2 \mathrm{H}), 2.68$ (ddd, $J=11.7,8.4,3.4 \mathrm{~Hz}, 2 \mathrm{H}), 2.05-$
$1.90(\mathrm{~m}, 2 \mathrm{H}), 1.80-1.6(\mathrm{~m}, 4 \mathrm{H}), 1.63-1.50(\mathrm{~m}, 4 \mathrm{H}), 1.44(\mathrm{dtd}, J=12.1,8.0,3.5 \mathrm{~Hz}, 2 \mathrm{H}), 1.03(\mathrm{t}, J=$ $\mathrm{Hz}, 4 \mathrm{H}$
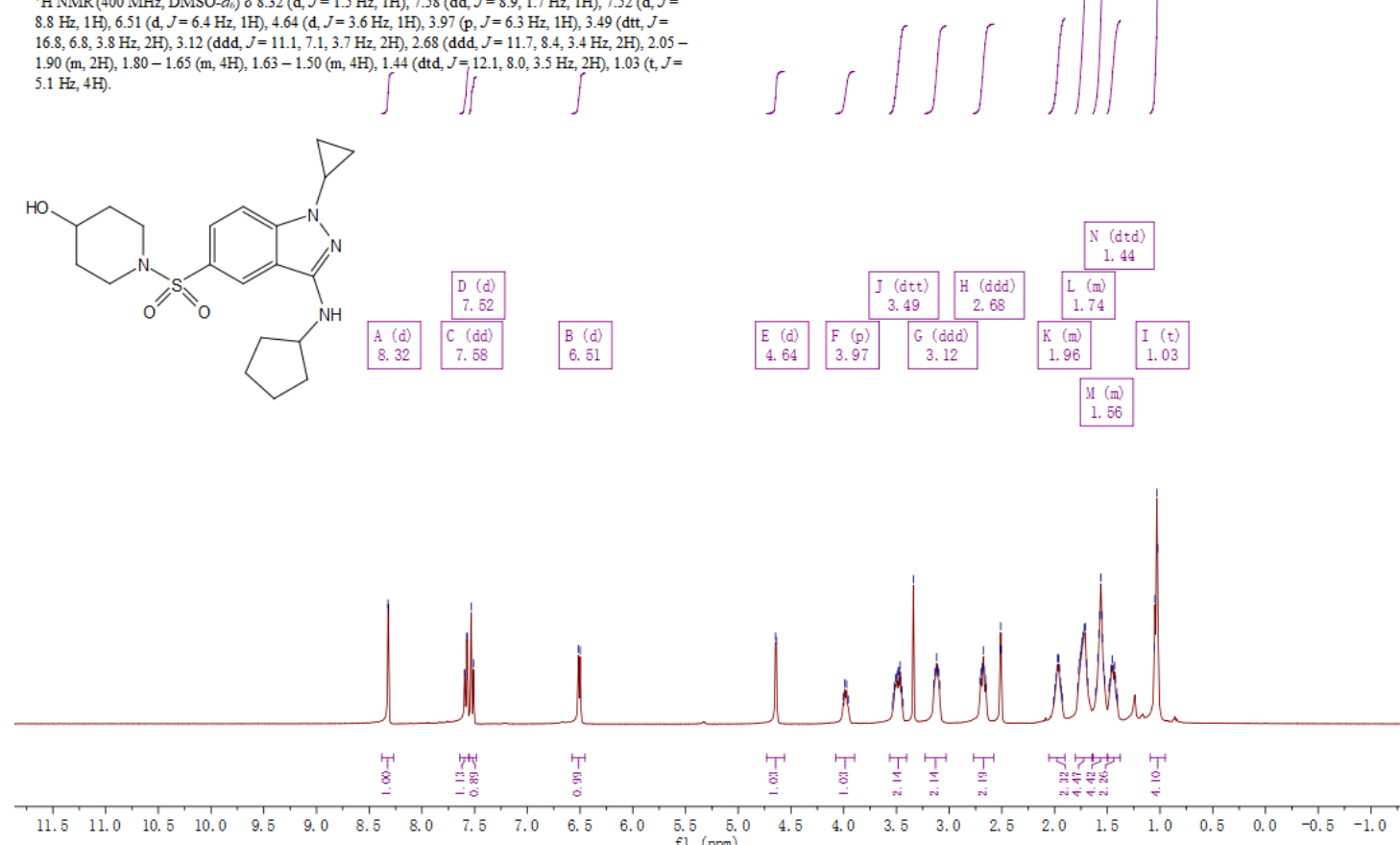
20190123-wct-1299-1-C. 10, fid
C13CPD DMSO [D: LNIR_DATA\} LG 25
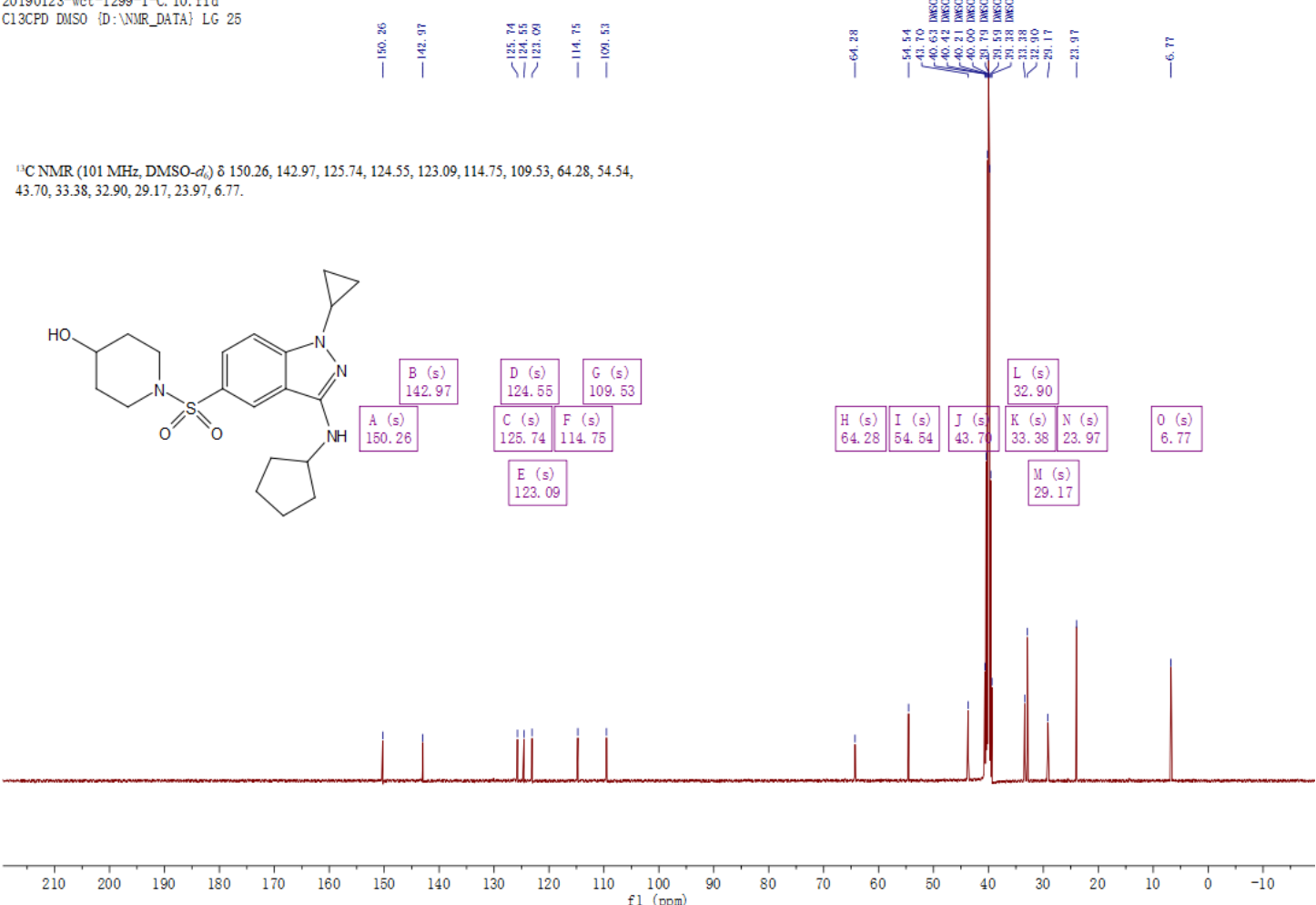


\section{Compound 29.}

20181122-wct-1257-2-H. 10, fid
PROTON DUSO \{D: $\backslash$ NMR_DATA\} LG 13

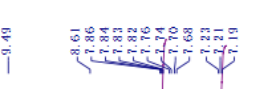

'H NMR (400 MHz, DMSO-d $) \delta 9.49(\mathrm{~s}, 1 \mathrm{H}), 8.61$ (s, $1 \mathrm{H}), 7.84$ (dd, $J=8.9,4.8 \mathrm{~Hz}, 2 \mathrm{H}), 7.7 \mathrm{~s}$ (d, $J=$ $8.9 \mathrm{~Hz}, 1 \mathrm{H}), 7.69(\mathrm{~d}, J=9.0 \mathrm{~Hz}, 1 \mathrm{H}), 7.21(\mathrm{t}, J=8.7 \mathrm{~Hz}, 2 \mathrm{H}), 6.04(\mathrm{p}, J=7.2 \mathrm{~Hz}, 1 \mathrm{H}), 5,12(\mathrm{t}, J=6.4$ $\mathrm{Hz}, 2 \mathrm{H}), 4.97(\mathrm{t}, J=7.0 \mathrm{~Hz}, 2 \mathrm{H}), 4.63(\mathrm{~d}, J=3.8 \mathrm{~Hz}, 1 \mathrm{H}), 3.50$ (dt, $J=8.2,3.9 \mathrm{~Hz}, 1 \mathrm{H}), 3.16$ (dt, $J=$
$11.2,4.6 \mathrm{~Hz}, 2 \mathrm{H}), 2.72(\mathrm{ddd}, J=12.0,8.9,3.3 \mathrm{~Hz}, 2 \mathrm{H}), 1.83-1.67(\mathrm{~m}, 2 \mathrm{H}), 1.44$ (dtd, $J=12.5,8.4,3.7$ $\mathrm{Hz}, 2 \mathrm{H})$

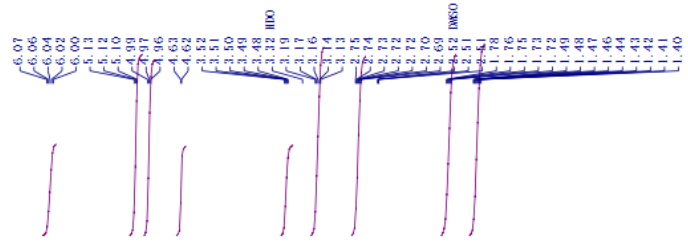<smiles>O=S(=O)(c1ccc2c(c1)c(Nc1ccc(F)cc1)nn2C1COC1)N1CCC(O)CC1</smiles>

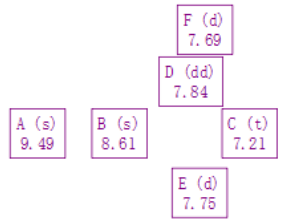

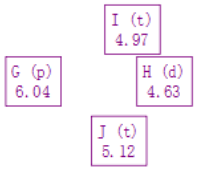

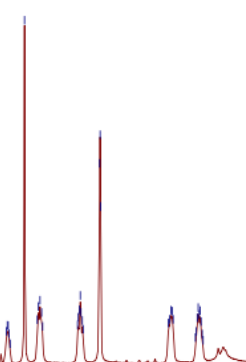

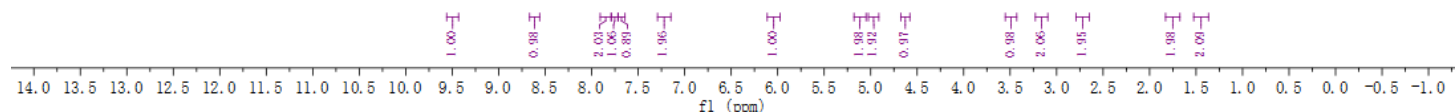

\section{3-HiCT-1257-2-c. 10. fid

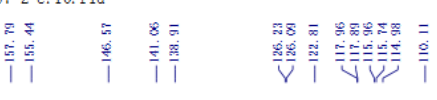

CNMR (101 MHz, DMSO- $\left.d_{d}\right) \delta 156.62$ (d, $\left.J=236.2 \mathrm{~Hz}\right), 146.57,141.06,138.91,126.23,126.09$, $122.81,117.93(\mathrm{~d}, J=7.3 \mathrm{~Hz}), 115.85(\mathrm{~d}, J=22.1 \mathrm{~Hz}), 114.98,110.11,76.91,64.24,51.42,43.72,33.35$.
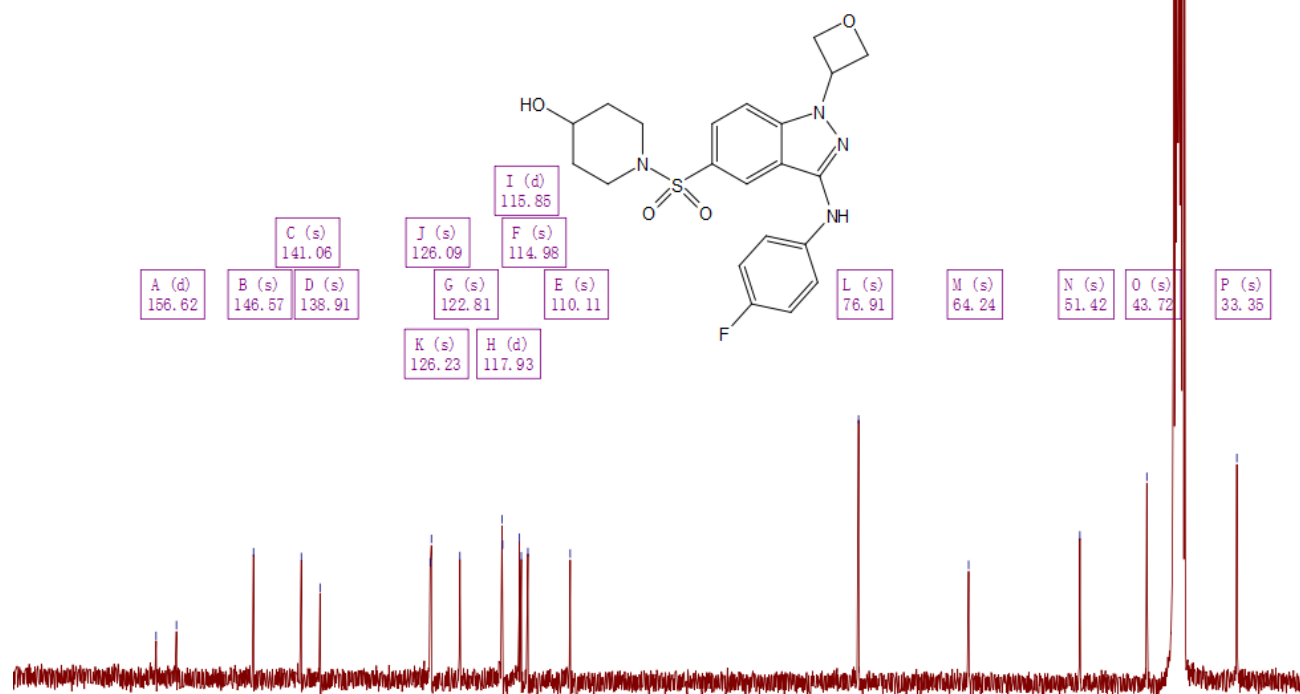

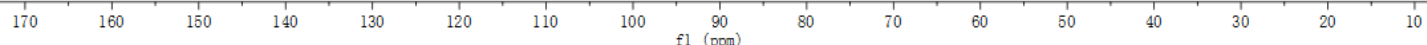




\section{Compound 30.}

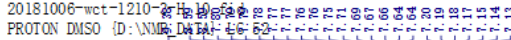

'H NMR (400 MHz, DNMSO-ds) $89.38(\mathrm{~s}, 1 \mathrm{H}), 8.59\left(\mathrm{~d}, \int=1.6 \mathrm{H} \neq, 1 \mathrm{H}\right), 7.81-7.73(\mathrm{~m}, 2 \mathrm{H}), 7.72-7.62$ $\iiint \iint$

$(\mathrm{m}, 2 \mathrm{H}), 7.16(\mathrm{t}, J=8.9 \mathrm{~Hz}, 2 \mathrm{H}), 4.66$ (d, $J=3.9 \mathrm{~Hz}, 1 \mathrm{H}), 4.36(q, J=7.1 \mathrm{~Hz}, 2 \mathrm{H}), 3.52$ (tt, $J=7.8,3.9$

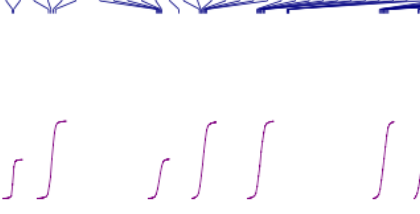

$\mathrm{Hz}, 1 \mathrm{H}), 3.18$ (ddd, $J=11.1,7.0,3.7$

$\mathrm{H}_{3} \mathrm{C}$
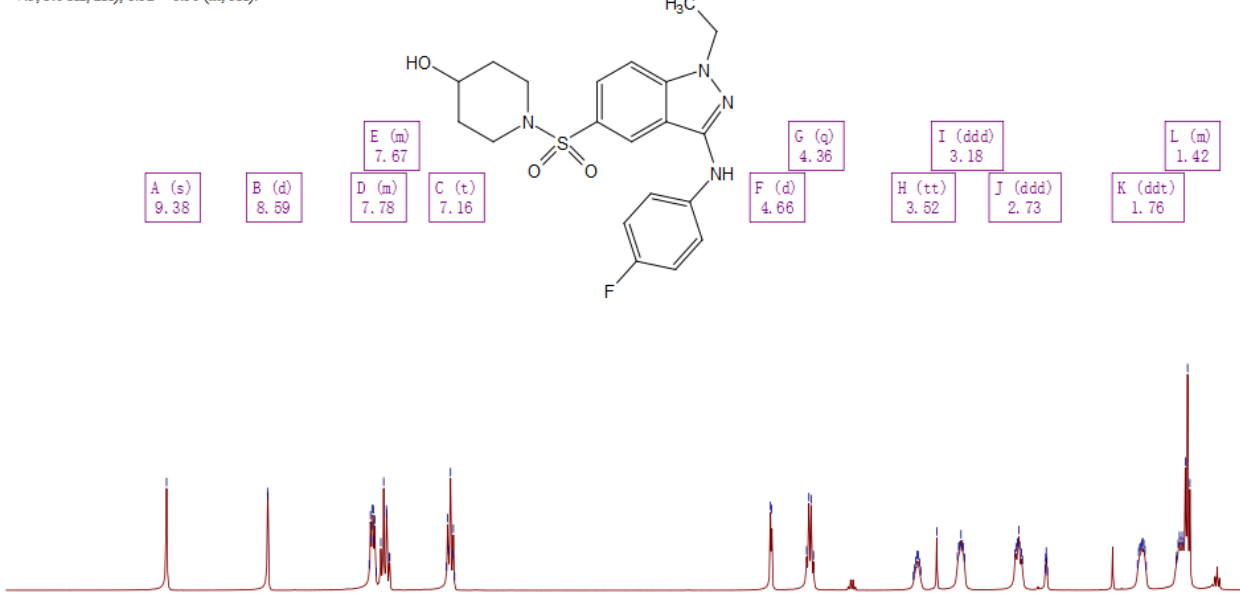

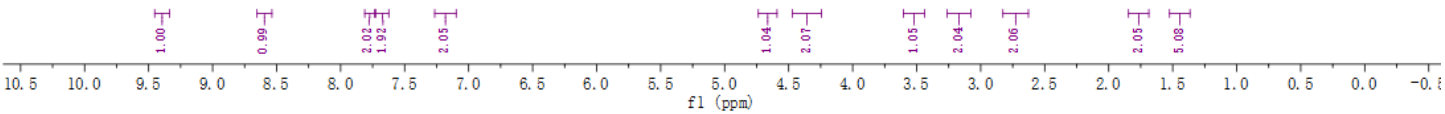

20181006-wct-1210-3-C. 20, fid
C13CPD DUSO \{D: \NIR_DATA\} LG 5

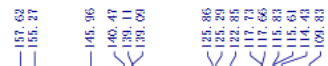

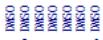

||

${ }^{13} \mathrm{CNMR}\left(101 \mathrm{MHz}\right.$, DMSO- $\left.d_{l}\right) \delta 156.44(\mathrm{~d}, J=235.7 \mathrm{~Hz}), 145.96,140.47,139.10(\mathrm{~d}, J=2.2 \mathrm{~Hz})$, $109,83,64,30,43,75$ $43.33,33.40,15.09$.
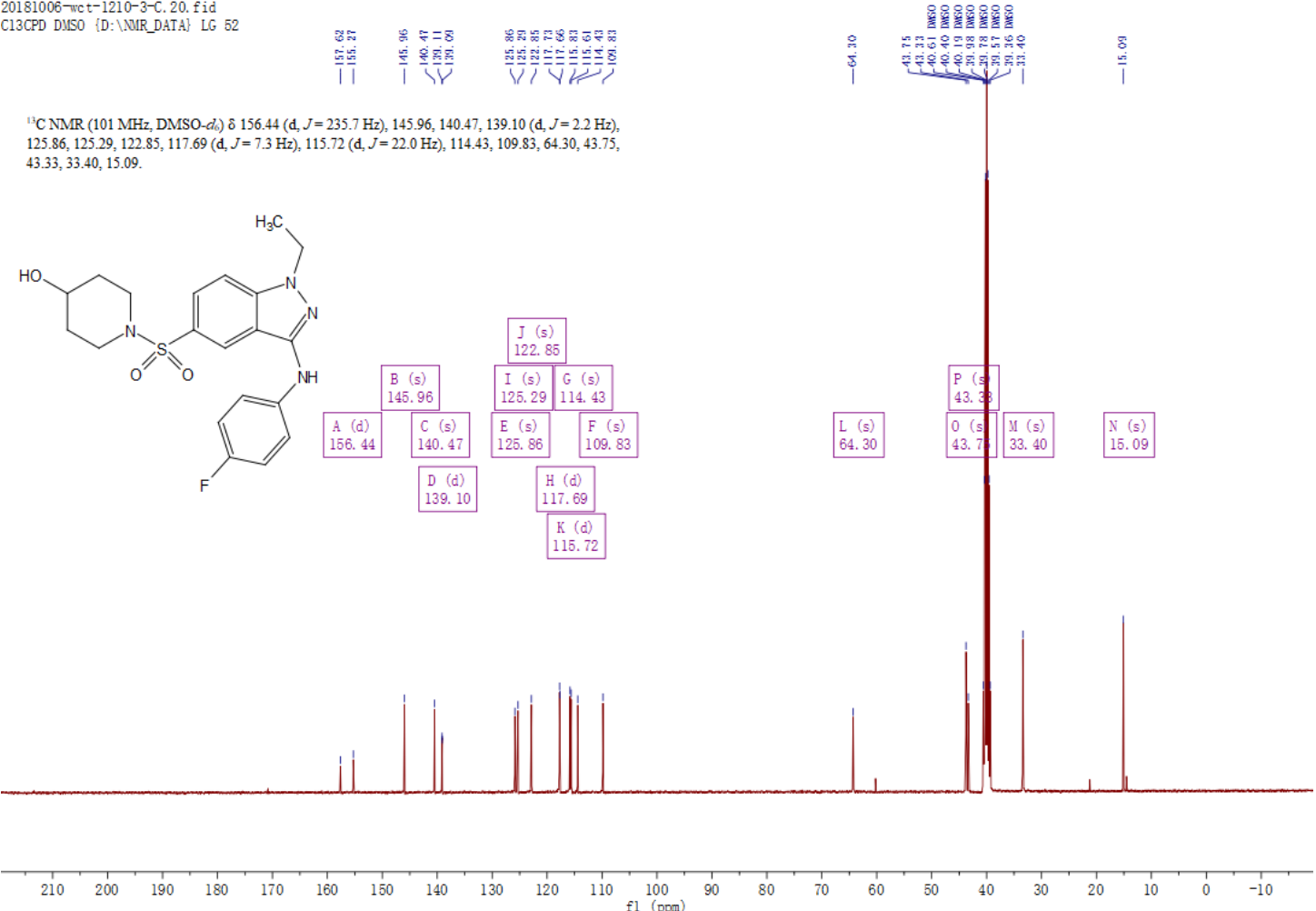


\section{Compound 31.}

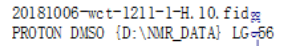

'H NMR (400 MHz, DMSO- $\left.d_{6}\right) \delta 9.38(\mathrm{~s}, 1 \mathrm{H}), 8.58(\mathrm{~d}, J=1.6 \mathrm{~Hz}, 1 \mathrm{H}), 7.82-7.74(\mathrm{~m}, 2 \mathrm{H}), 7.72(\mathrm{~d}, J$

$=9.0 \mathrm{~Hz}, 1 \mathrm{H}), 7.64(\mathrm{dd}, J=8.9,1.7 \mathrm{~Hz}, 1 \mathrm{H}), 7.17(\mathrm{t}, J=8.9 \mathrm{~Hz}, 2 \mathrm{H}), 4.92(\mathrm{p}, J=6.5 \mathrm{~Hz}, 1 \mathrm{H}), 4.66(\mathrm{~d}, J$

$=3.9 \mathrm{~Hz}, 1 \mathrm{H}), 3.52(\mathrm{tt}, J=7.6,3.7 \mathrm{~Hz}, 1 \mathrm{H}), 3.17$ (ddd, $J=11.1,7.0,3.8 \mathrm{~Hz}, 2 \mathrm{H}$ ), 2.73 (ddd, $J=11.7$,

$8.5,3.4 \mathrm{~Hz}, 2 \mathrm{H}), 1.77$ (ddt, $J=13.7,7.1,3.2 \mathrm{~Hz}, 2 \mathrm{H}), 1.54-1.41(\mathrm{~m}, 8 \mathrm{H})$
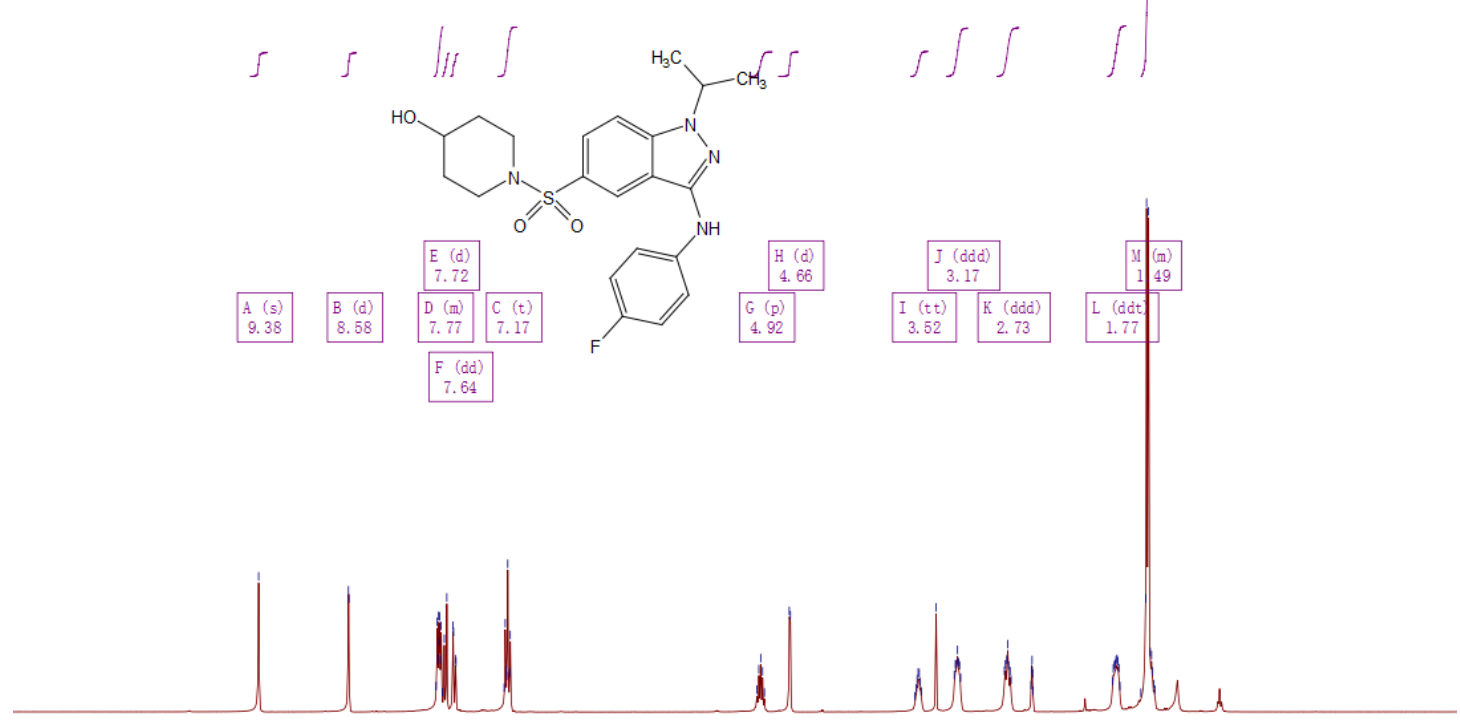

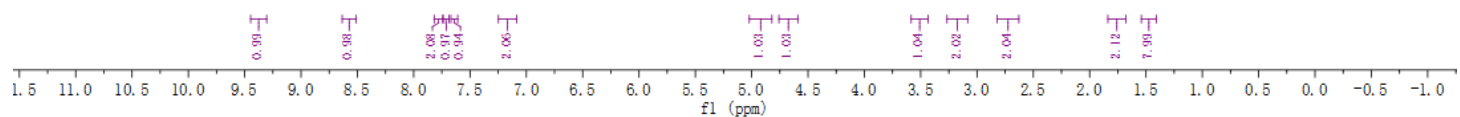
20181006-wct-1211-1-C. 10. fid

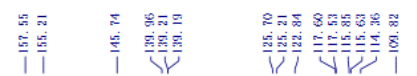

${ }^{13} \mathrm{C} \mathrm{NMR}\left(101 \mathrm{MHz}, \mathrm{DMSO}-\mathrm{d}_{\mathrm{s}}\right) \delta 156.38$ (d, $\left.J=235.4 \mathrm{~Hz}\right), 145.74,139.96,139.20$ (d, $J=1.9 \mathrm{~Hz}$ ), (25.70, 125.21, 122.84, 117.57 (d, $J=7.4 \mathrm{~Hz}), 115.74$ (d, $J=22.2 \mathrm{~Hz}), 114.36,109.82,64.27,49.62$ $43.75,33.39,22.26$

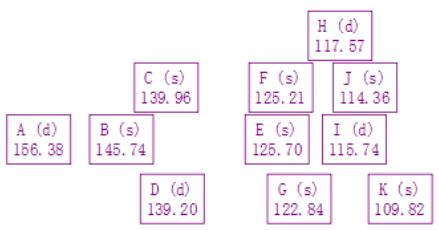<smiles>CC(C)n1nc(Nc2ccc(F)cc2)c2cc(S(=O)(=O)N3CCC(O)CC3)ccc21</smiles>

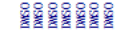

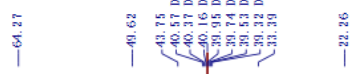
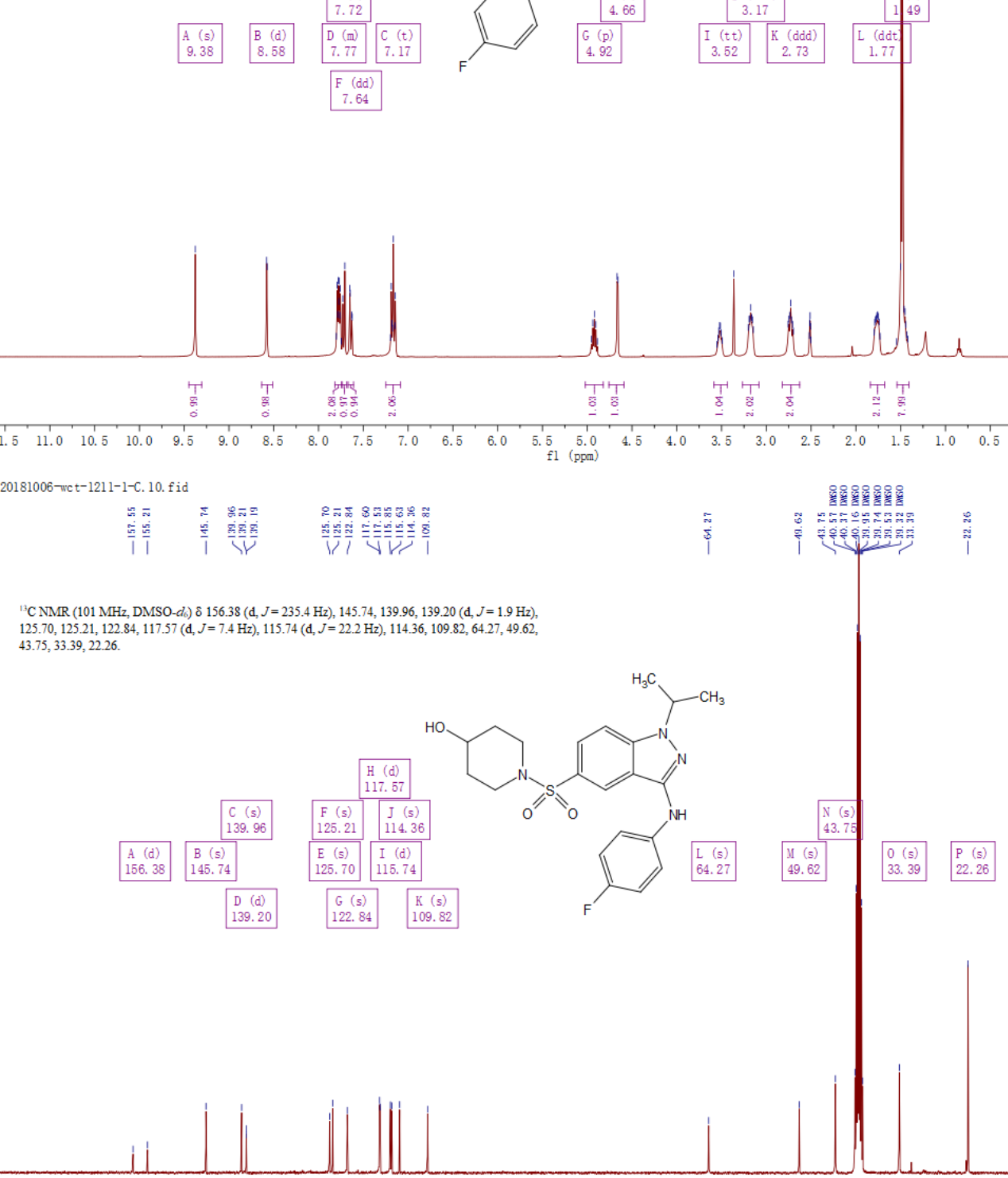


\section{Compound 32.}

20190104-wct-1290-1-H. 10. fid

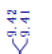

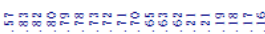

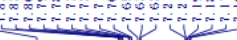

요료

蕰要

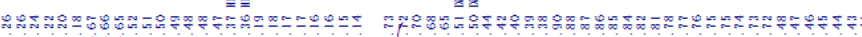

'H NMR (400 MHz, DMSO- $\left.d_{\text {l) }}\right) 89.42$ (d, $\left.J=2.6 \mathrm{~Hz}, 1 \mathrm{H}\right), 8.57(\mathrm{~s}, 1 \mathrm{H}), 7.81$ (dt, $\left.J=8.0,3.6 \mathrm{~Hz}, 2 \mathrm{H}\right)$, $7.72(\mathrm{dd}, J=9.0,2.6 \mathrm{~Hz}, 1 \mathrm{H}), 7.64(\mathrm{~d}, J=8.5 \mathrm{~Hz}, 1 \mathrm{H}), 7.19$ (td, $J=9.0,2.7 \mathrm{~Hz}, 2 \mathrm{H}), 5.22(\mathrm{p}, J=9.0 \mathrm{~Hz}$, 1H), $4.66(\mathrm{t}, J=3.3 \mathrm{~Hz}, 1 \mathrm{H}), 3.49(\mathrm{tt}, J=7.4,3.8 \mathrm{~Hz}, 1 \mathrm{H}), 3.24-3.08(\mathrm{~m}, 2 \mathrm{H}), 2.69(\mathrm{q}, J=9.5,8.6 \mathrm{~Hz}$, $4 \mathrm{H}), 2.47-2.34(\mathrm{~m}, 2 \mathrm{H}), 1.95-1.80(\mathrm{~m}, 2 \mathrm{H}), 1.75$ (ddd, $J=13.6,7.0,3.5 \mathrm{~Hz}, 2 \mathrm{H}), 1.45$ (ddt, $J=16.7$ $11.9,5.6 \mathrm{~Hz}, 2 \mathrm{H})$

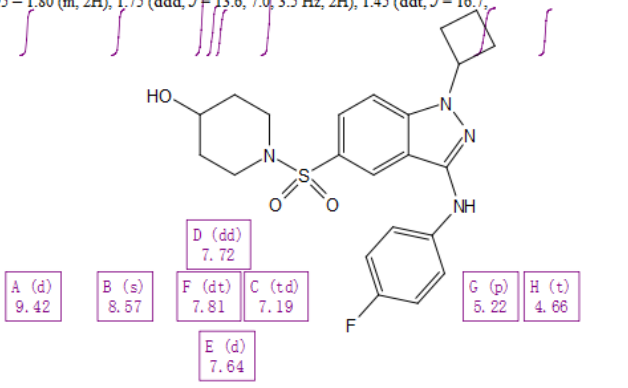

\begin{tabular}{|c|c|c|}
\hline & \begin{tabular}{|cc} 
L & (m) \\
2.41
\end{tabular} & $\begin{array}{c}N \text { (ddd) } \\
1.75\end{array}$ \\
\hline \begin{tabular}{|cc} 
& $(t t)$ \\
3.49
\end{tabular} & $\begin{array}{ll}\mathrm{K} & (\mathrm{q}) \\
2.69\end{array}$ & \begin{tabular}{|l|}
$M(\mathrm{~m})$ \\
1.86
\end{tabular} \\
\hline
\end{tabular}

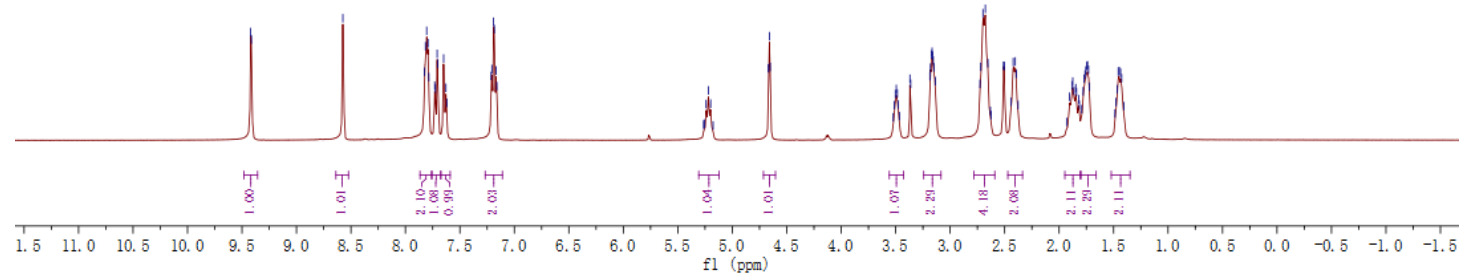

20190104-wct-1290-1-C.10fid
C13CPD DUSO \{D:INUR_DATA\} LG 34.

${ }^{13} \mathrm{C} \mathrm{NMR}\left(101 \mathrm{MHz}, \mathrm{DMSO}-d_{\mathrm{l}}\right) \delta 156.47$ (d, $\left.J=235.6 \mathrm{~Hz}\right), 145.98,140.24,139.11$ (d, $J=1.9 \mathrm{~Hz}$,

$125.88,125.55,122.81,117.69$ (d, $J=7.4 \mathrm{~Hz}), 115.79(\mathrm{~d}, J=22.1 \mathrm{~Hz}), 114.61,109.95,64.31,51.71$

$43.76,33.40,29.85,14.94$
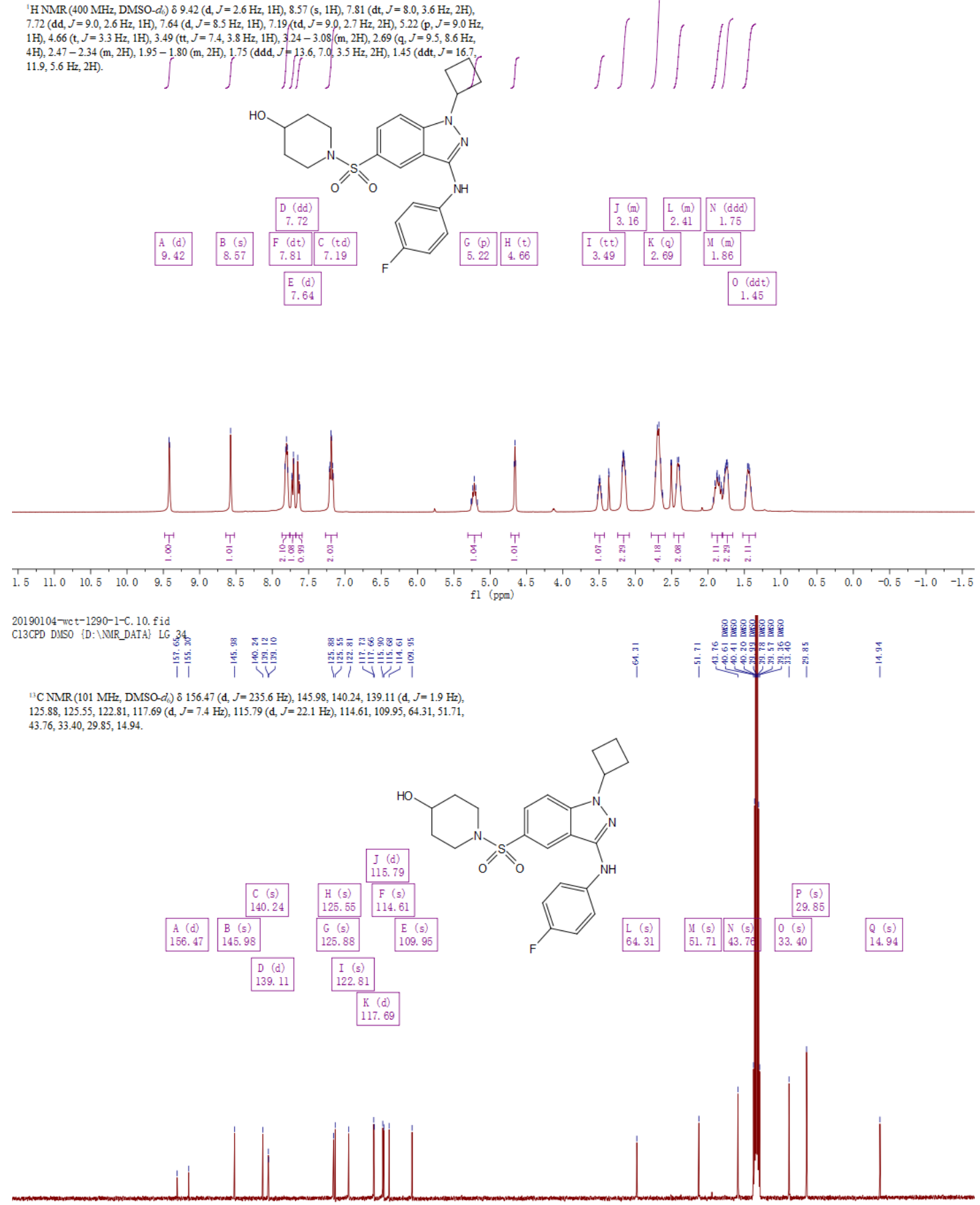

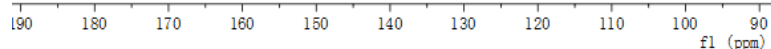




\section{Compound 33.}

20180928-wct-1206-1-H. 10 fid
PROTON DUSO \{D: NUR_DATA\} TYF 36

'H NMR (400 MHz, DMSO-d $d_{\text {) }} \delta 9.39$ (s, $\left.1 \mathrm{H}\right), 8.60$ (d, $\left.J=1.4 \mathrm{~Hz}, 1 \mathrm{H}\right), 7.75$ (dd, $J=9.1,4.7 \mathrm{~Hz}, 2 \mathrm{H}$ ) $7.73-7.67(\mathrm{~m}, 2 \mathrm{H}), 7.17(\mathrm{t}, J=8.9 \mathrm{~Hz}, 2 \mathrm{H}), 3.74-3.59(\mathrm{~m}, 5 \mathrm{H}), 2.97-2.80(\mathrm{~m}, 4 \mathrm{H}), 1.14(\mathrm{~d}, J=5.3$ $\mathrm{Hz}, 4 \mathrm{H})$.

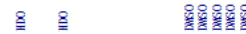

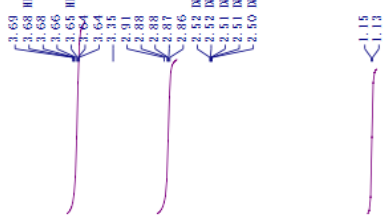

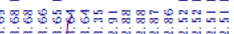

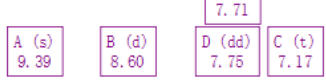<smiles>O=S(=O)(c1ccc2c(c1)c(Nc1ccc(F)cc1)nn2C1CC1)N1CCOCC1</smiles>

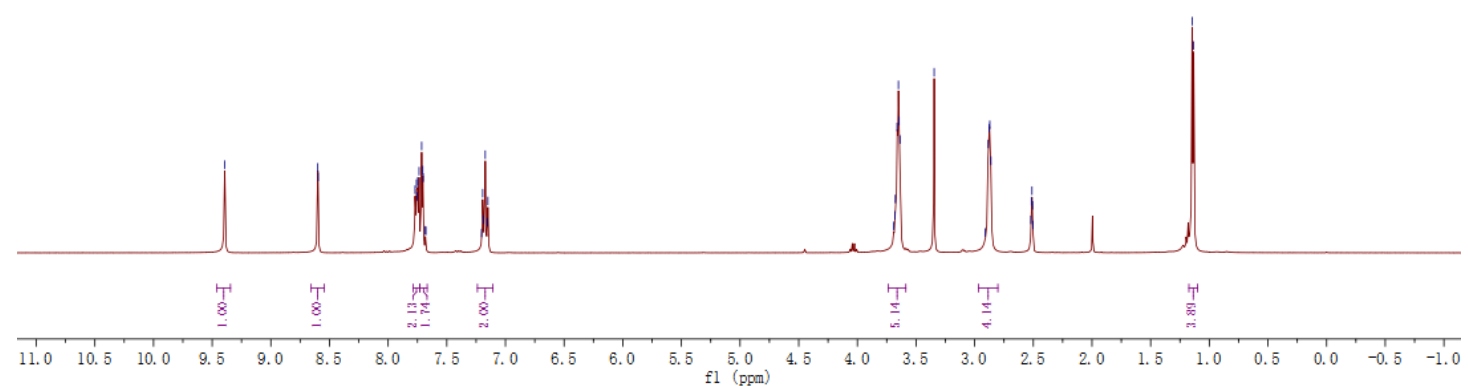

20180928-wct-1206-1-C. 10. fid
C13CPD DUSO \{D: \NUR_DATA\} LG

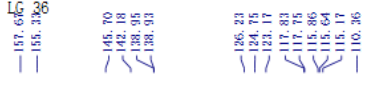

${ }_{13}^{13} \mathrm{C} \mathrm{NMR}\left(101 \mathrm{MHz}, \mathrm{DMSO}-\mathrm{d}_{\mathrm{s}}\right) \delta 156.50(\mathrm{~d}, J=235.7 \mathrm{~Hz}), 145.70,142.18,138.94(\mathrm{~d}, J=2.0 \mathrm{~Hz})$ $126.23,124.75,123.17,117.79$ (d, $J=7.4 \mathrm{~Hz}), 115.75(\mathrm{~d}, J=22.0 \mathrm{~Hz}), 115.17,110.36,65.74,46.42$ $29.59,6.87$.
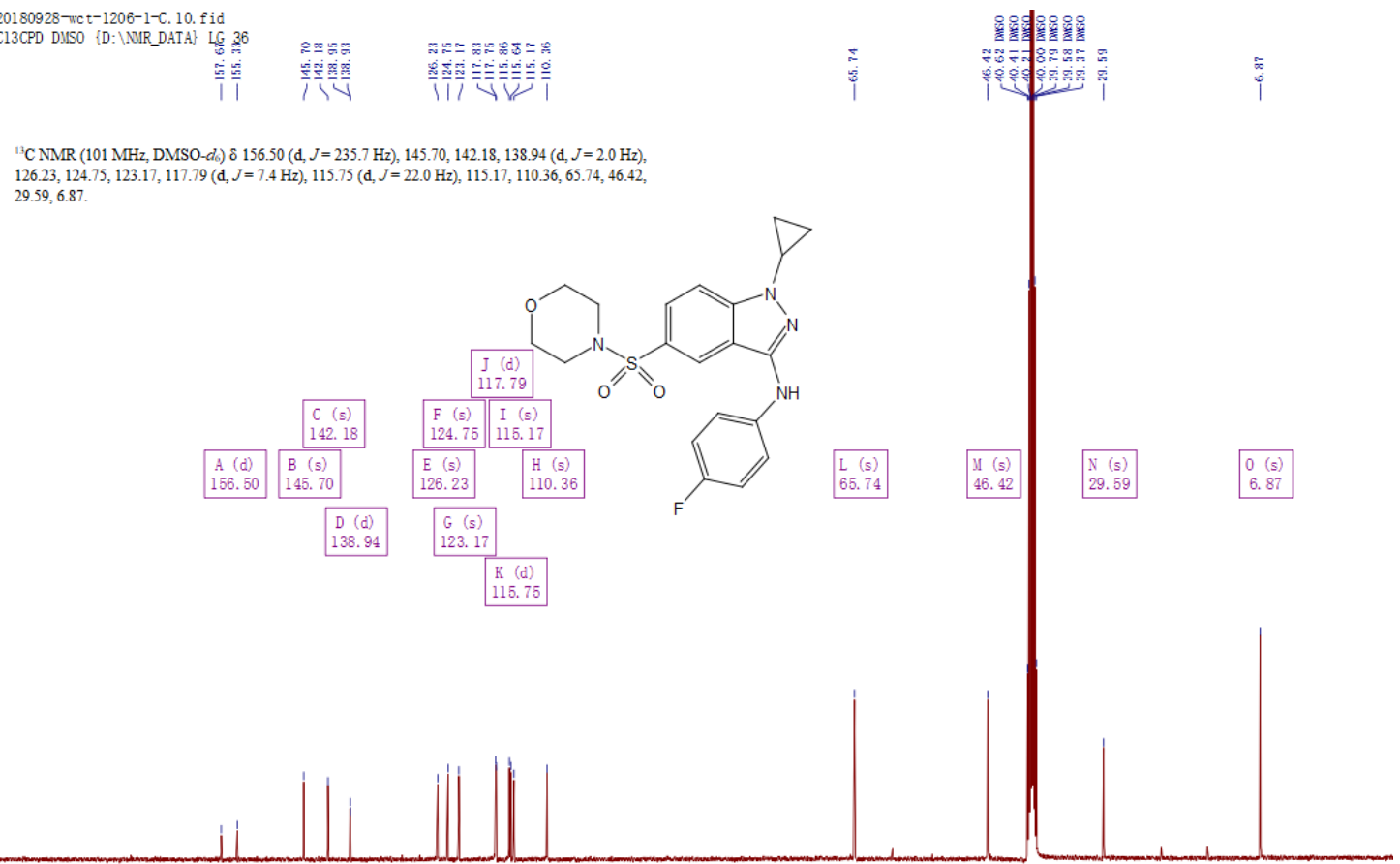

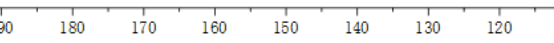

$\cos ^{1}$

70

$60 \quad 50$

40 


\section{Compound 34.}

20181030-wct-1230-2-H. 10, fid

然

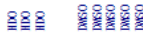

'H NMR (400 MHz, DMSO- $\left.d_{\mathrm{s}}\right) \delta 9.38(\mathrm{~s}, 1 \mathrm{H}), 8.58(\mathrm{~d}, J=1.2 \mathrm{~Hz}, 1 \mathrm{H}), 7.80-7.72(\mathrm{~m}, 2 \mathrm{H}), 7.70(\mathrm{~d}, J$

$=1.7 \mathrm{~Hz}, 2 \mathrm{H}), 7.24-7.10(\mathrm{~m}, 2 \mathrm{H}), 4.98(\mathrm{~d}, J=4.8 \mathrm{~Hz}, 1 \mathrm{H}), 3.65(\mathrm{p}, J=5.3 \mathrm{~Hz}, 1 \mathrm{H}), 3.55(\mathrm{tq}, J=8.8$,

$4.1 \mathrm{~Hz}, 1 \mathrm{H}), 3.46(\mathrm{dd}, J=10.9,4.2 \mathrm{~Hz}, 1 \mathrm{H}), 3.30(\mathrm{~s}, 1 \mathrm{H}), 2.11(\mathrm{td}, J=11.1,2.5 \mathrm{~Hz}, 1 \mathrm{H}), 2.18-2.07(\mathrm{~m}$

1H), $1.81-1.65(\mathrm{~m}, 2 \mathrm{H}), 1.47(\mathrm{qd}, J=11.7,10.6,3.7 \mathrm{~Hz}, 1 \mathrm{H}), 1.13(\mathrm{~d}, J=5.3 \mathrm{~Hz}, 4 \mathrm{H}), 1.11-1.00(\mathrm{~m}$

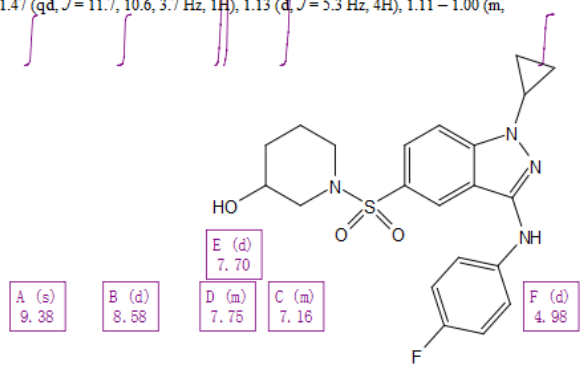

$\iint /$
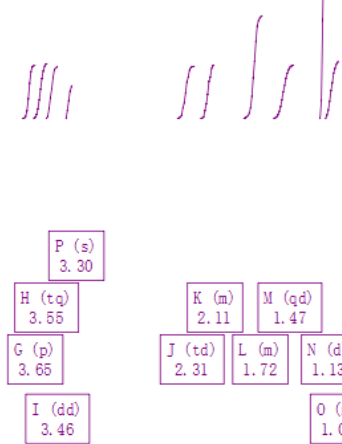

\begin{tabular}{|c|c|c|}
\hline $\begin{array}{l}\mathrm{K} \text { (m } \\
2.11\end{array}$ & & \\
\hline $\begin{array}{c}\text { (td) } \\
2.31\end{array}$ & $\begin{array}{l}\text { L (m) } \\
1.72\end{array}$ & $\begin{array}{r}\text { N (d) } \\
1.13\end{array}$ \\
\hline
\end{tabular}

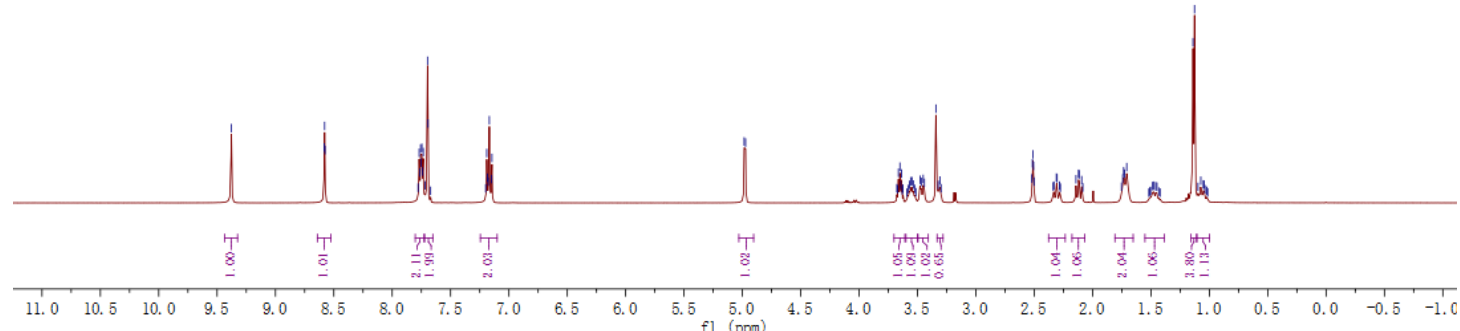

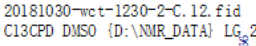

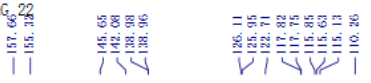

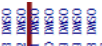

C NMR (101 MHz, DMSO- $\left.d_{l}\right) \delta 156.49$ (d, $\left.J=235.5 \mathrm{~Hz}\right), 145.65,142.08,138.97$ (d, $J=2.0 \mathrm{~Hz}$ ), $126.11,125.95,122.71,117.78(\mathrm{~d}, J=7.3 \mathrm{~Hz}), 115.74(\mathrm{~d}, J=22.1 \mathrm{~Hz}), 115.13,110.26,65.50,53.14$, $46.33,32.43,29.57,22.72,6.86$.

$$
6.86 \text {. }
$$

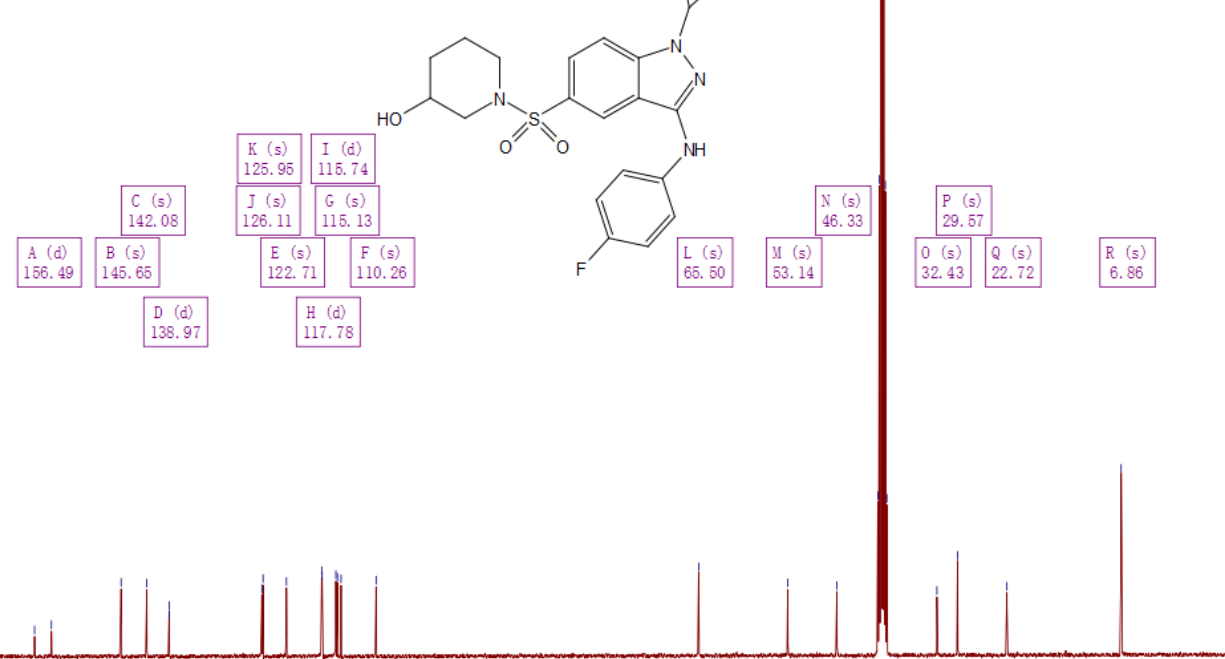

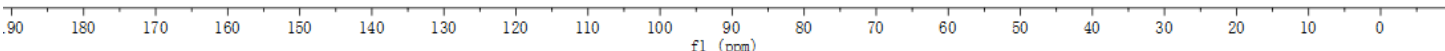




\section{Compound 35.}

20181121-wct-1245-1-H. 10, fid
PROTON DUSO \{D_NIR_DATA\} LG 13

i

${ }^{\prime} \mathrm{H} \mathrm{NMR}\left(400 \mathrm{MHz}, \mathrm{DMSO}-d_{\mathrm{d}}\right) \delta 12.52(\mathrm{~s}, 1 \mathrm{H}), 9.37(\mathrm{~s}, 1 \mathrm{H}), 8.66(\mathrm{~s}, 1 \mathrm{H}), 7.77$ (dt, $J=8.925 .7 \mathrm{~Hz}$,

$3 \mathrm{H}), 7.69(\mathrm{~d}, J=8.9 \mathrm{~Hz}, 1 \mathrm{H}), 7.17(\mathrm{t}, J=8.8 \mathrm{~Hz}, 2 \mathrm{H}), 3.65(\mathrm{p}, J=5.3 \mathrm{~Hz}, 1 \mathrm{H}), 3.43-3.28(\mathrm{~m}, 2 \mathrm{H}), 32$.
$-3.12(\mathrm{~m}, 2 \mathrm{H}), 2.94(\mathrm{p}, J=7.3 \mathrm{~Hz}, 1 \mathrm{H}), 1.97(\mathrm{dd}, J=13.0,7.1 \mathrm{~Hz}, 1 \mathrm{H}), 1.88(\mathrm{td}, J=14.6,12.8,7.9 \mathrm{~Hz}$,

$1 \mathrm{H}), 1.14(\mathrm{~d}, J=5,3 \mathrm{H}, 4 \mathrm{H})$

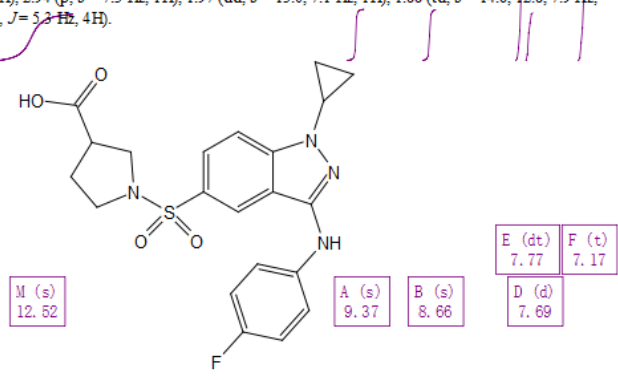

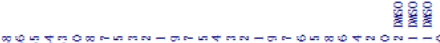

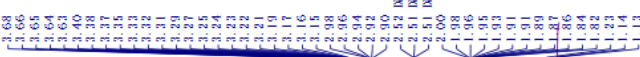
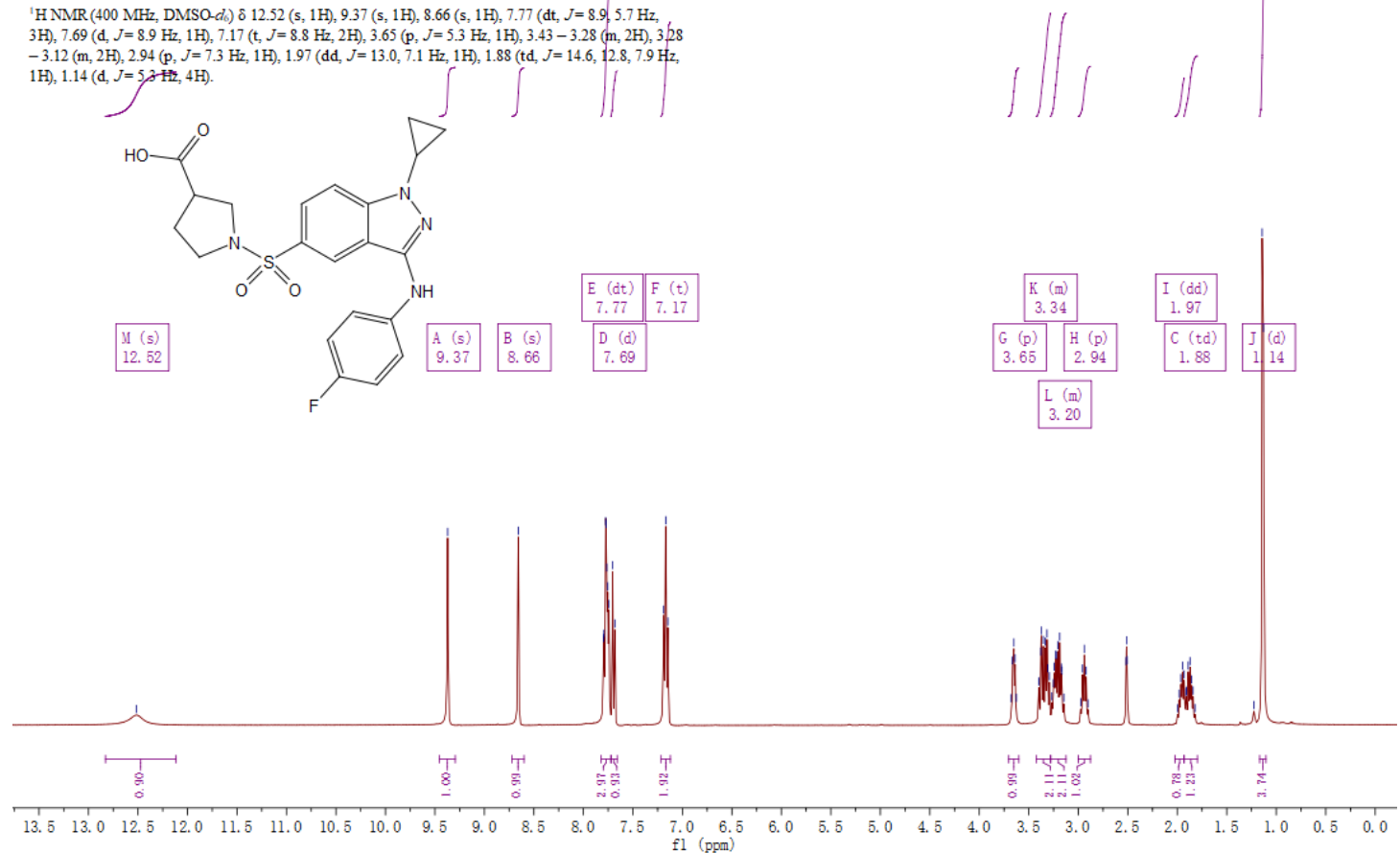

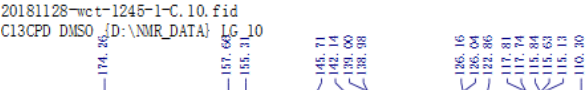

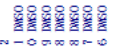

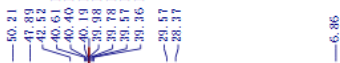

${ }^{3} \mathrm{C}$ NMR $\left(101 \mathrm{MHz}\right.$, DMSO- $\left.d_{s}\right)$ 8 174.26, 156.48 (d, $\left.J=235.7 \mathrm{~Hz}\right), 145.71,142.14,138.99$ (d, $J=2.1$

z), 126.16, 126.04, 122.86, 117.77 (d, $J=7.5 \mathrm{~Hz}), 115.74(\mathrm{~d}, J=22.0 \mathrm{~Hz}), 115.13,110.30,50.21,47.8$

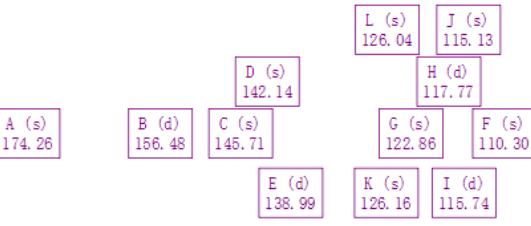<smiles>O=C(O)C1CCN(S(=O)(=O)c2ccc3c(c2)c(Nc2ccc(F)cc2)nn3C2CC2)C1</smiles>

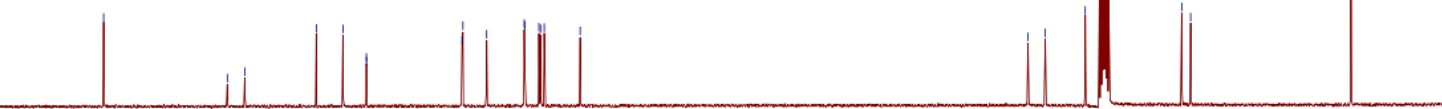

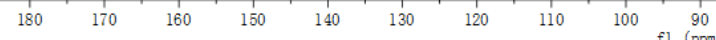




\section{Compound 36.}

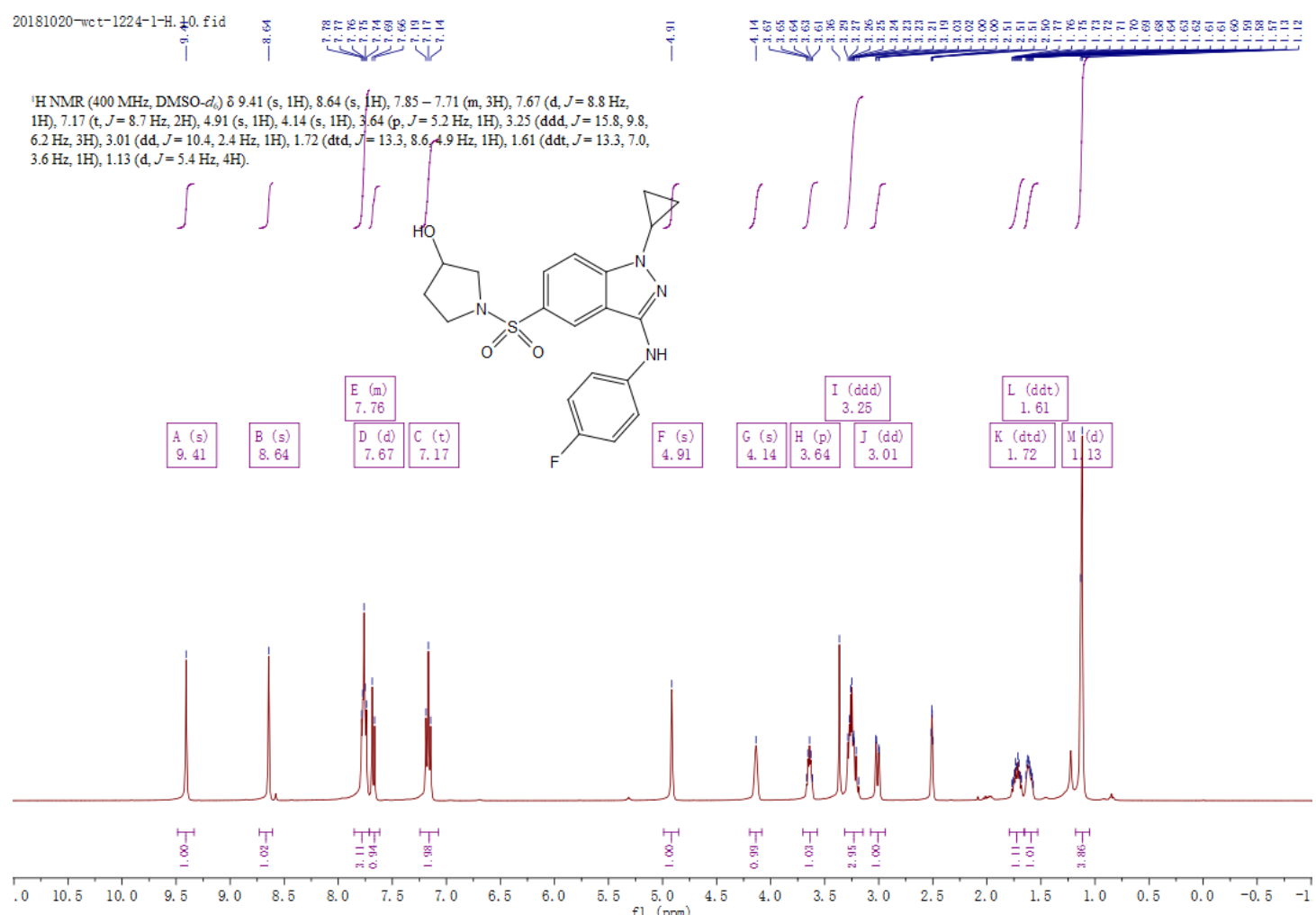

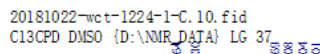

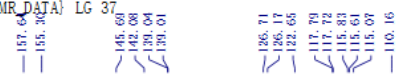

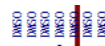

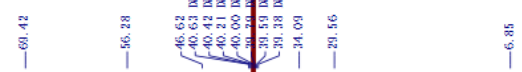

${ }_{13} \mathrm{C} \mathrm{NMR}$ (101 MHz, DMSO- $d$ ) $\delta 156.47$ (d, $J=235.5 \mathrm{~Hz}$ ), $145.69,14208,139.02$ (d, $\left.J=2.2 \mathrm{~Hz}\right)$, $126.71,126.17,122.65,117.75(\mathrm{~d}, J=7.4 \mathrm{~Hz}), 115.72(\mathrm{~d}, J=22.2 \mathrm{~Hz}), 115.07,110.16,69.42,56.28$ $46.62,34.09,29.56,6.85$.
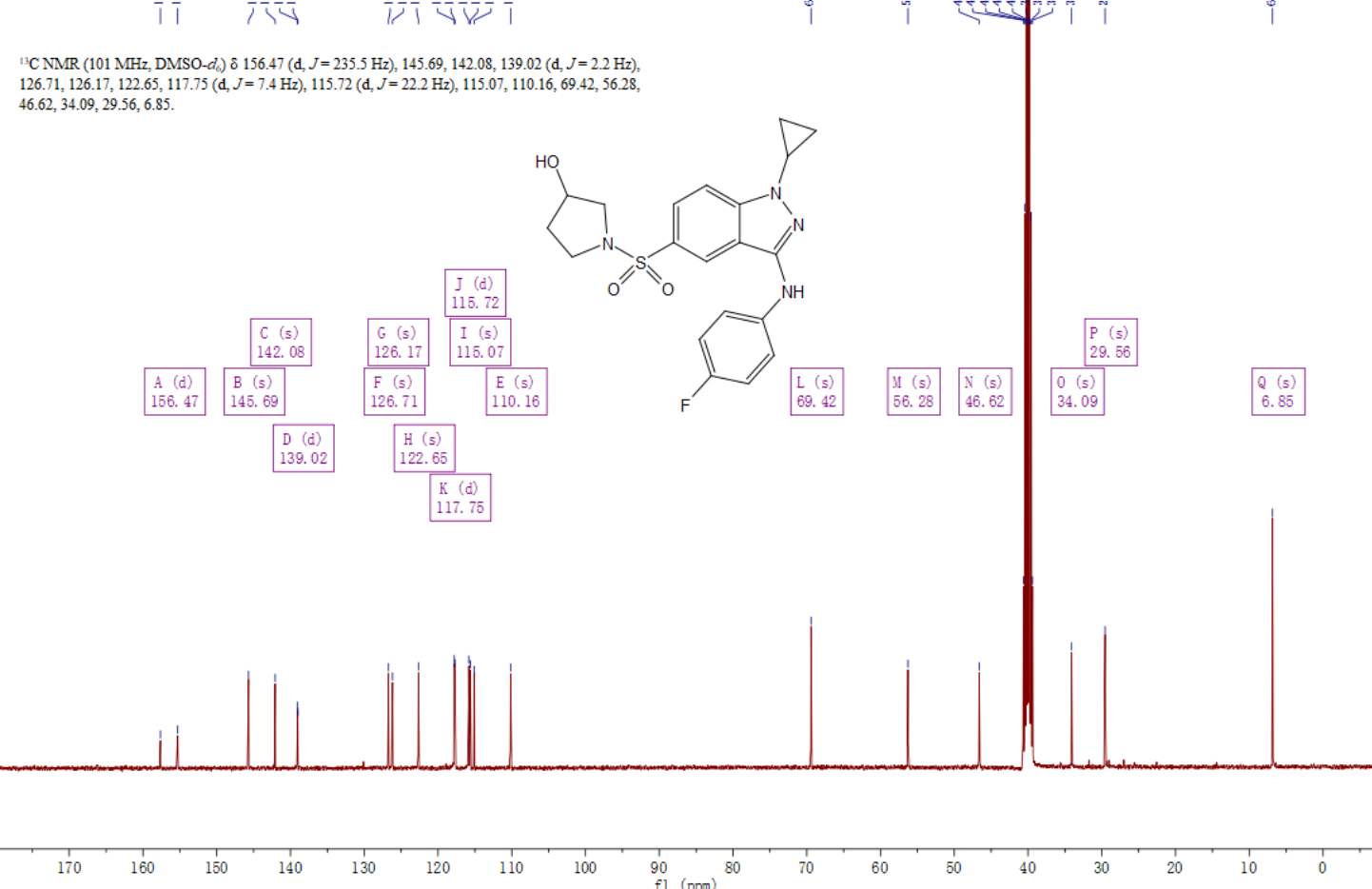


\section{Compound 37.}

$$
\text { 20181029-wct-1225-1-H. 10. fid }
$$

'H NMR (400 MHz, DMSO- $\left.d_{6}\right) \delta 9.39(\mathrm{~s}, 1 \mathrm{H}), 8.57(\mathrm{~d}, J=1.4 \mathrm{~Hz}, 1 \mathrm{H}), 7.84-7.70(\mathrm{~m}, 3 \mathrm{H}), 7.68(\mathrm{~d}, J$ $=1.2 \mathrm{~Hz}, 2 \mathrm{H}), 7.16(\mathrm{t}, J=8.9 \mathrm{~Hz}, 2 \mathrm{H}), 4.49(\mathrm{t}, J=5.1 \mathrm{~Hz}, 1 \mathrm{H}), 3.75-3.58(\mathrm{~m}, 3 \mathrm{H}), 3.27-3.10(\mathrm{~m}, 2 \mathrm{H})$, $=1.1 \mathrm{~Hz}, 2 \mathrm{H}), 7.16(\mathrm{t}, \mathrm{Jz}=8.9 \mathrm{~Hz}, 2 \mathrm{H}), 4.49(\mathrm{t}, J=5.1 \mathrm{~Hz}, 1 \mathrm{H}, 3.7 .3-3.18(\mathrm{~m}, 3 \mathrm{H})$,
$2.17(\mathrm{td}, J=11.7,2.4 \mathrm{~Hz}, 2 \mathrm{H}, 1.70(\mathrm{dd}, J=12.9,3.3 \mathrm{~Hz}, 2 \mathrm{H}), 1.20-1.07(\mathrm{~m}, 6 \mathrm{H})$.

$$
\iiint \int^{1}
$$

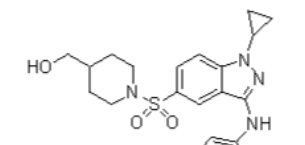

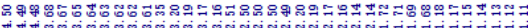
ن
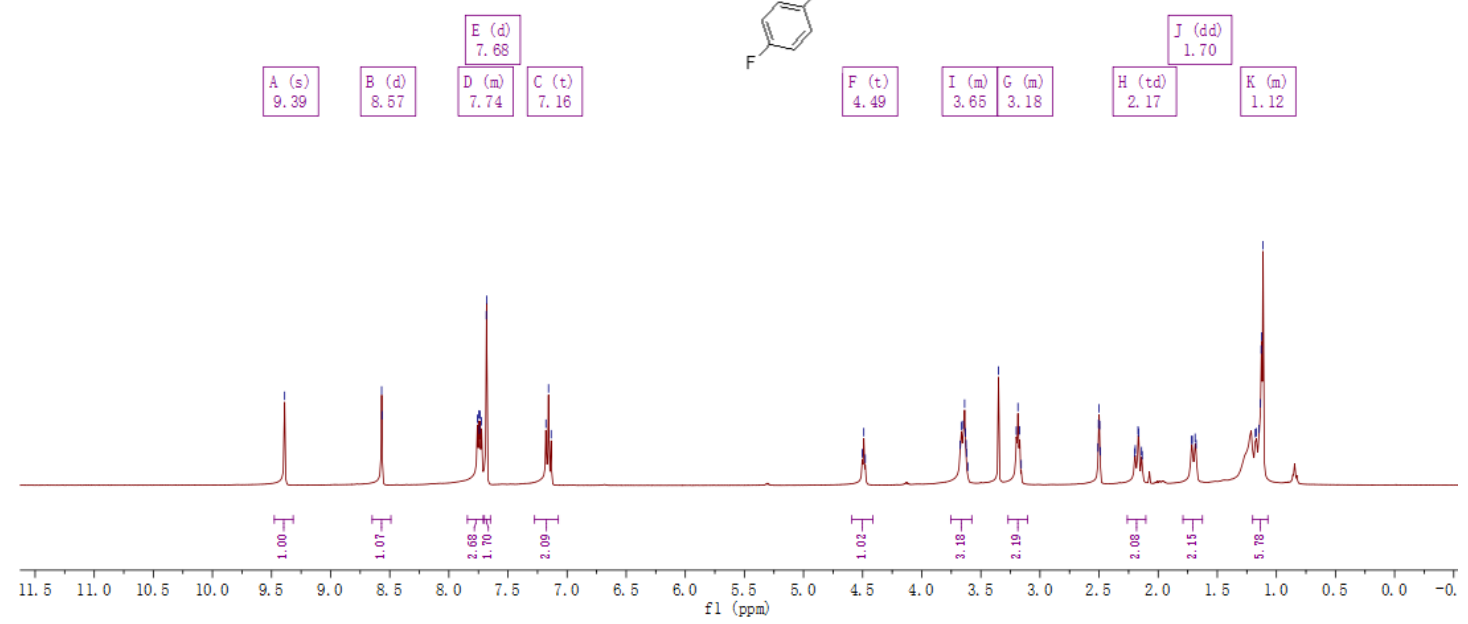

20181031-wct-1225-1-C. 10. fid

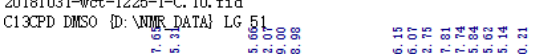

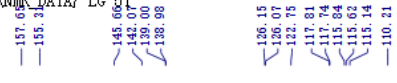

${ }^{13} \mathrm{CNMR}\left(101 \mathrm{MHz}, \mathrm{DMSO}-d_{\mathrm{s}}\right) \delta 156.48(\mathrm{~d}, J=235.9 \mathrm{~Hz}), 145.66,142.07,138.99$ (d, $\left.J=2.2 \mathrm{~Hz}\right)$,

$126.11(\mathrm{~d}, J=8.7 \mathrm{~Hz}), 122.75,117.77(\mathrm{~d}, J=7.3 \mathrm{~Hz}), 115.84,115.62,115.14,110.21,65.67,46.39,37.66$,

29.57, 28.31, 6.86.

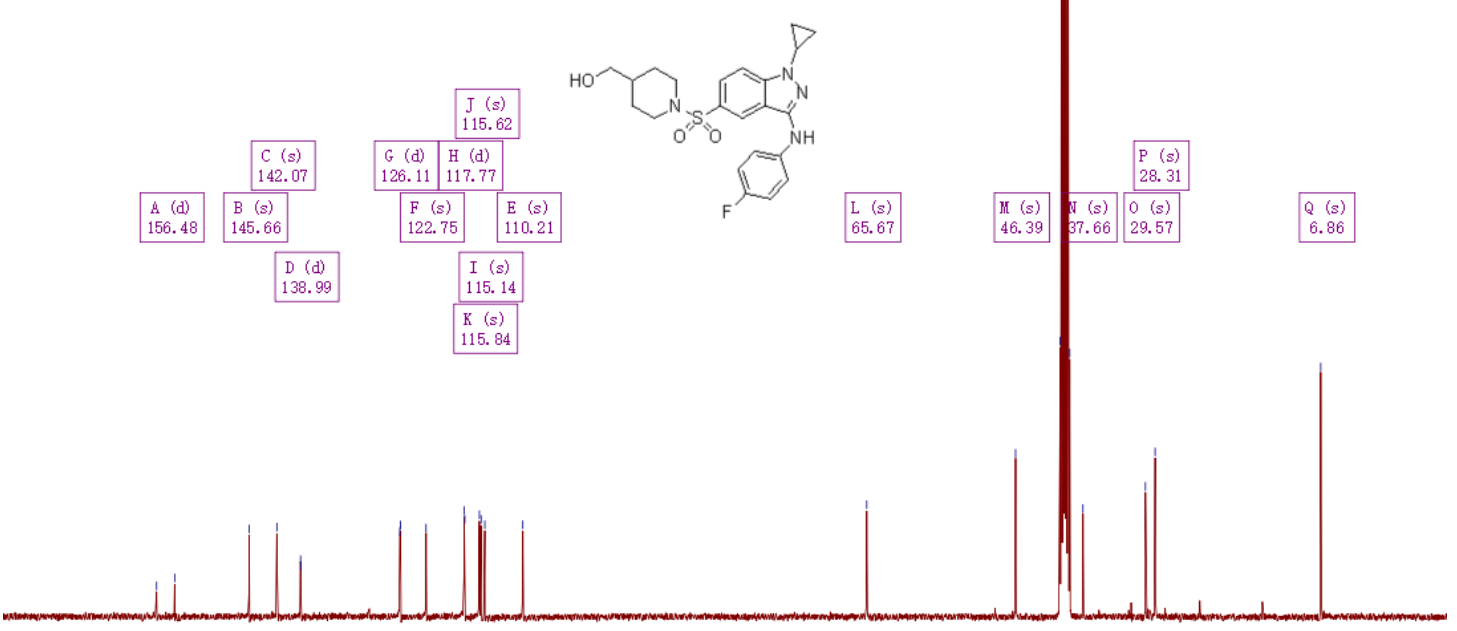

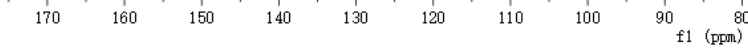




\section{Compound 38.}

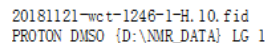
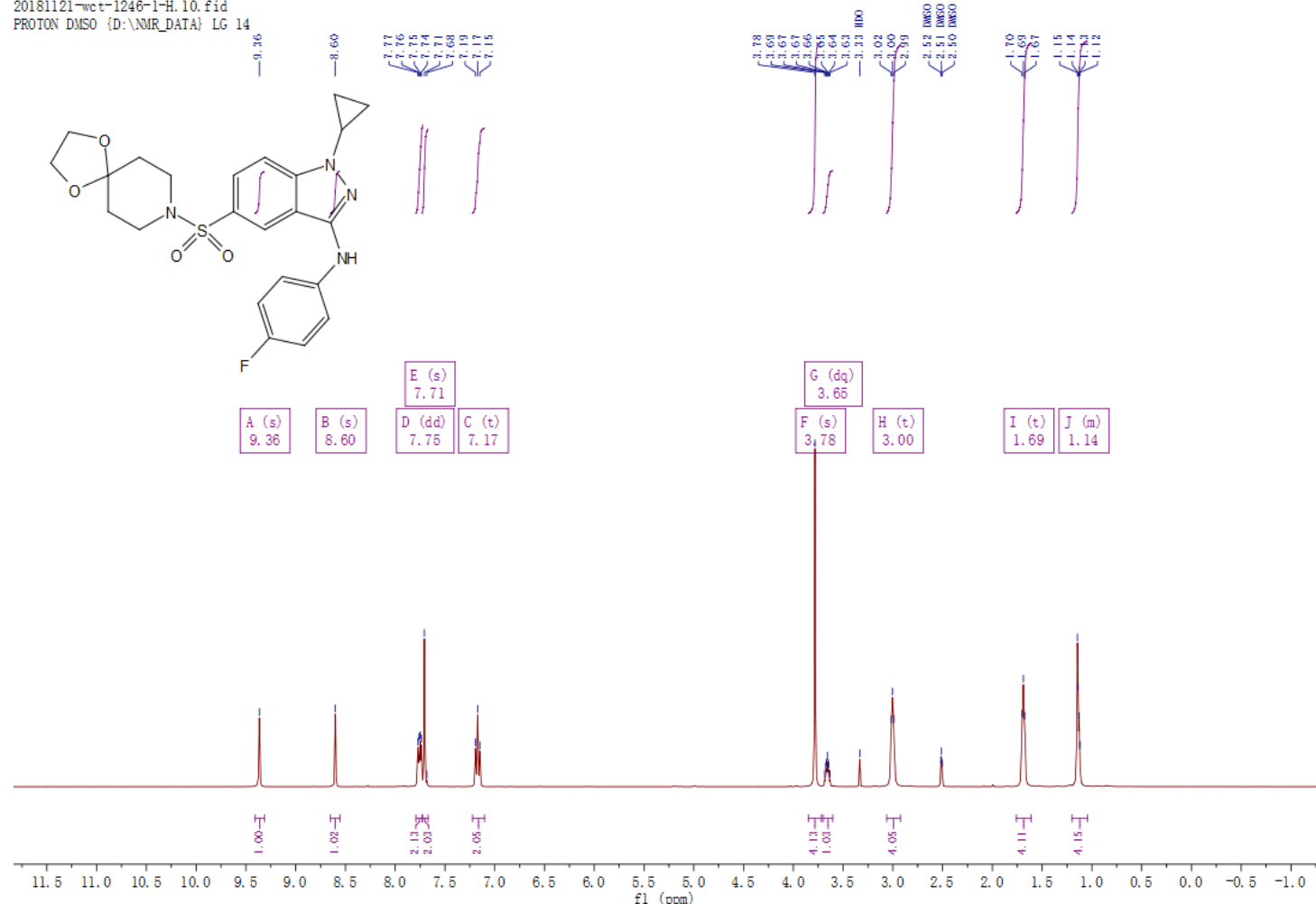

20181128-wct-1246-1-C. 10. fid

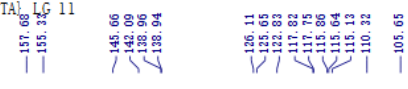

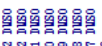

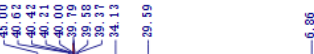

${ }^{13} \mathrm{C}$ NMR (101 MHz, DMSO- $\left.d_{3}\right) \delta 156.51$ (d, $\left.J=235.9 \mathrm{~Hz}\right), 145.66,142.09,138.95$ (d, $J=2.1 \mathrm{~Hz}$ ),

$126.11,125.65,122.83,117.79(\mathrm{~d}, J=7.4 \mathrm{~Hz}), 115.75(\mathrm{~d}, J=22.2 \mathrm{~Hz}), 115.13,110.32,105.65,64.20$

$45.00,34.13,29.59,6.86$<smiles>CNc1nn(C2CC2)c2ccc(S(=O)(=O)N3CCC4(CC3)OCCO4)cc12</smiles>

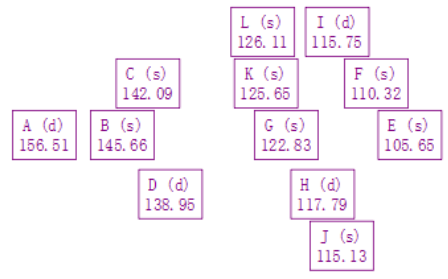<smiles>Nc1ccc(F)cc1</smiles>
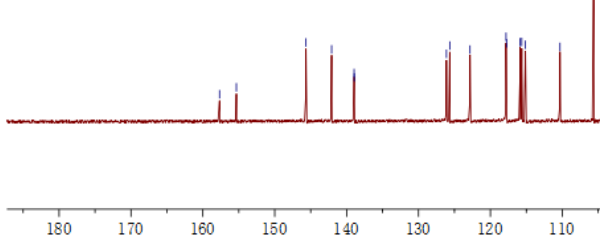

$120 \quad 110 \quad 100$ $\mathrm{fl}^{90}(\mathrm{ppm}) \mathrm{8}$

$\begin{array}{lll}10 & 1 & 1 \\ & & 1\end{array}$ 


\section{Compound 39.}

20181121-wct-1247-1-H. 10, fid
PROTON DUSO [D: (NMR.DATA] LG 15

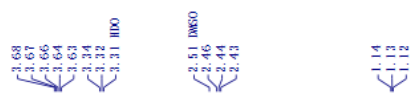

H NMR (400 MHz, DMSO-dl) $89.40(\mathrm{~s}, 1 \mathrm{H}), 8.67(\mathrm{~s}, 1 \mathrm{H}), 7.82-7.66(\mathrm{~m}, 4 \mathrm{H}), 7.16(\mathrm{t}, J=8.7 \mathrm{~Hz}$

$2 \mathrm{H}), 3.66(\mathrm{p}, J=5.4 \mathrm{~Hz}, 1 \mathrm{H}), 3.33(\mathrm{~d}, J=6.6 \mathrm{~Hz}, 4 \mathrm{H}), 2.44(\mathrm{t}, J=6.1 \mathrm{~Hz}, 4 \mathrm{H}), 1.19-1.07(\mathrm{~m}, 4 \mathrm{H})$

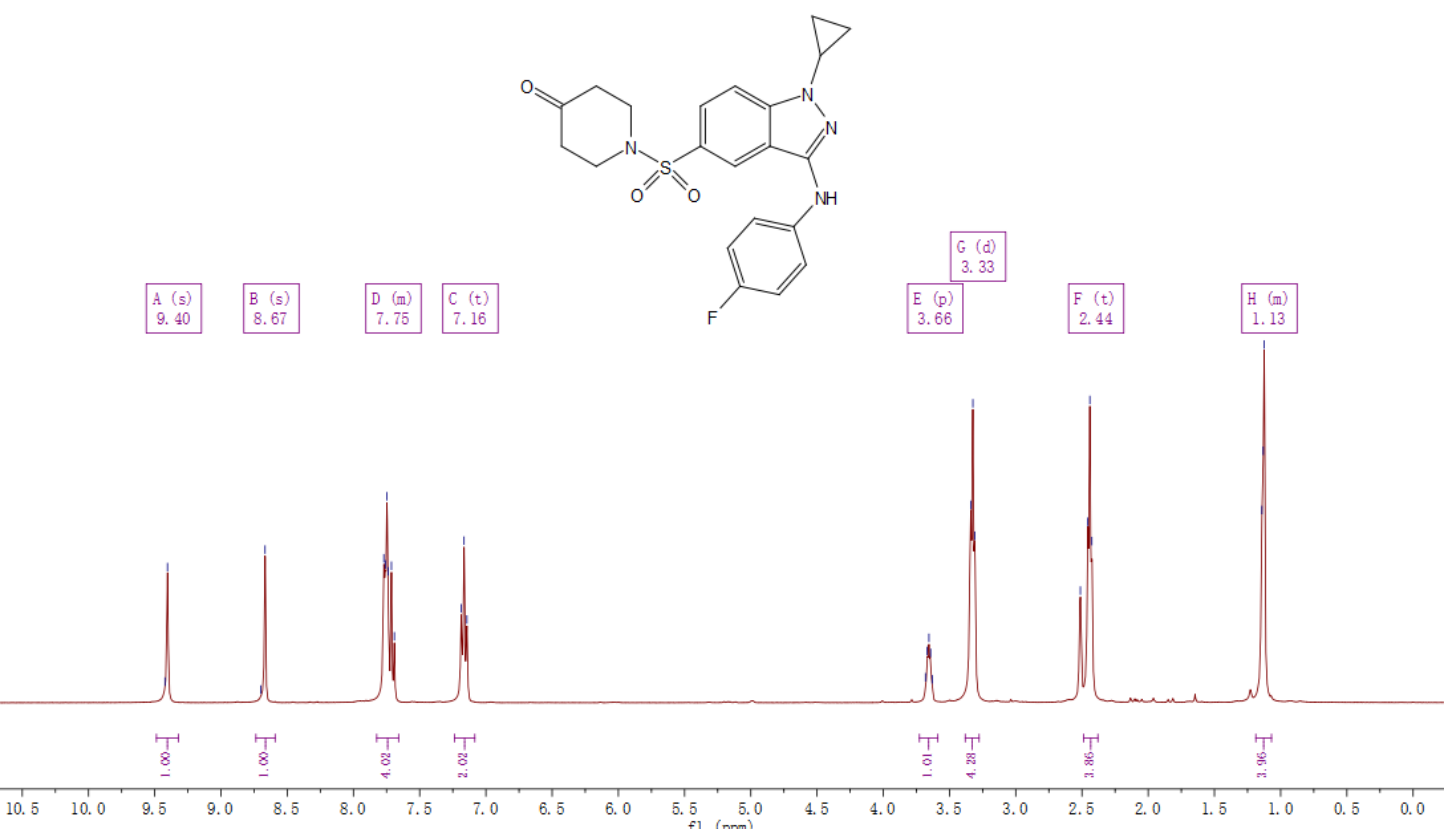

20181127-wct-1247-1-C. 10,fid
C13CPD DYSO [D: $\backslash$ NIR_DATA\} LG 7

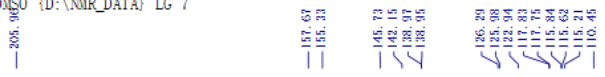

${ }^{13} \mathrm{C} \mathrm{NMR}\left(101 \mathrm{MHz}, \mathrm{DMSO}-d_{\text {) }}\right) \delta 205.96,156.50$ (d, $J=235.6 \mathrm{~Hz}$ ), 145.73, $142.15,138.96$ (d, $J=2.1$ Hz) $12629,125.98,122.94,117.79(\mathrm{~d}, J=7.4 \mathrm{~Hz}), 115.73(\mathrm{~d}, J=22.2 \mathrm{~Hz}), 115.21,110.45,45.63,29.58$ 6.86.

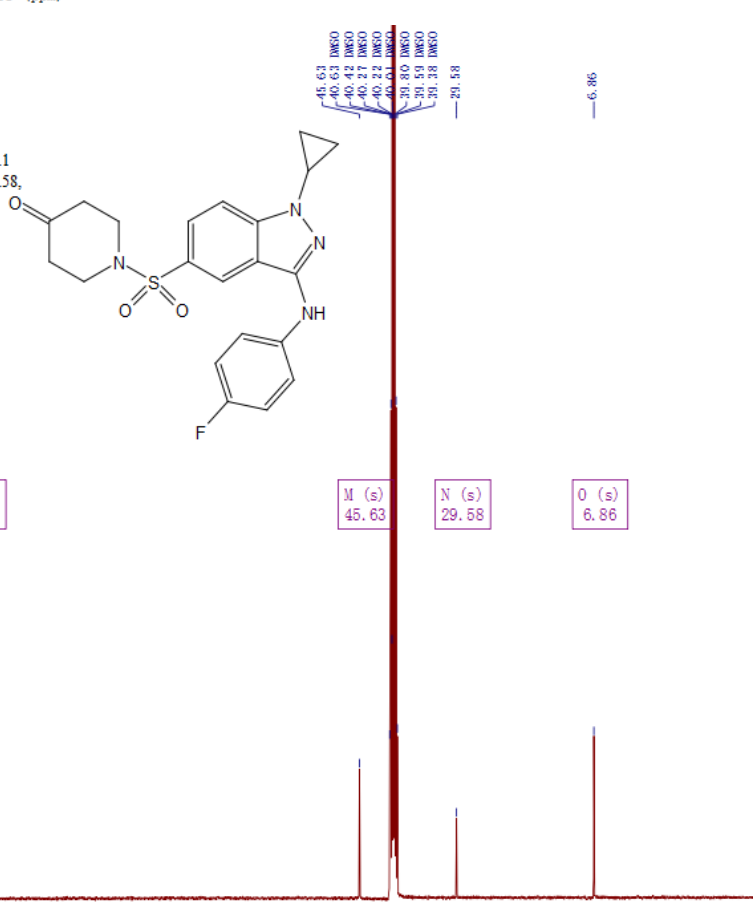

A $(s)$
205.96

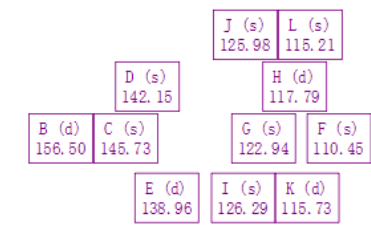
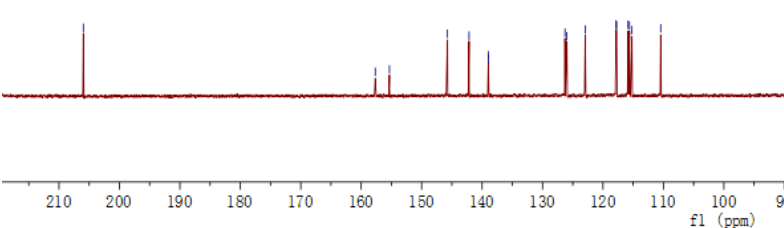


\section{Compound 40.}

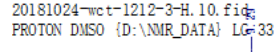

'H NMR (400 MHz, DMSO-d $\mathrm{d}_{\mathrm{s}} \delta 9.29(\mathrm{~s}, 1 \mathrm{H}), 8.59(\mathrm{~d}, J=1.5 \mathrm{~Hz}, 1 \mathrm{H}), 7.78-7.67(\mathrm{~m}, 2 \mathrm{H}), 7.67-7.60$ $(\mathrm{m}, 1 \mathrm{H}), 7.54(\mathrm{dd}, J=6.9,2.8 \mathrm{~Hz}, 1 \mathrm{H}), 7.10(\mathrm{t}, J=9.2 \mathrm{~Hz}, 1 \mathrm{H}), 3.75-3.59(\mathrm{~m}, 5 \mathrm{H}), 2.87$ (dd, $J=5.6$, , Hz $4 \mathrm{H}, 225$ (s, 3H) 1.14 (d, $J=53 \mathrm{~Hz}, 4 \mathrm{H}$ )

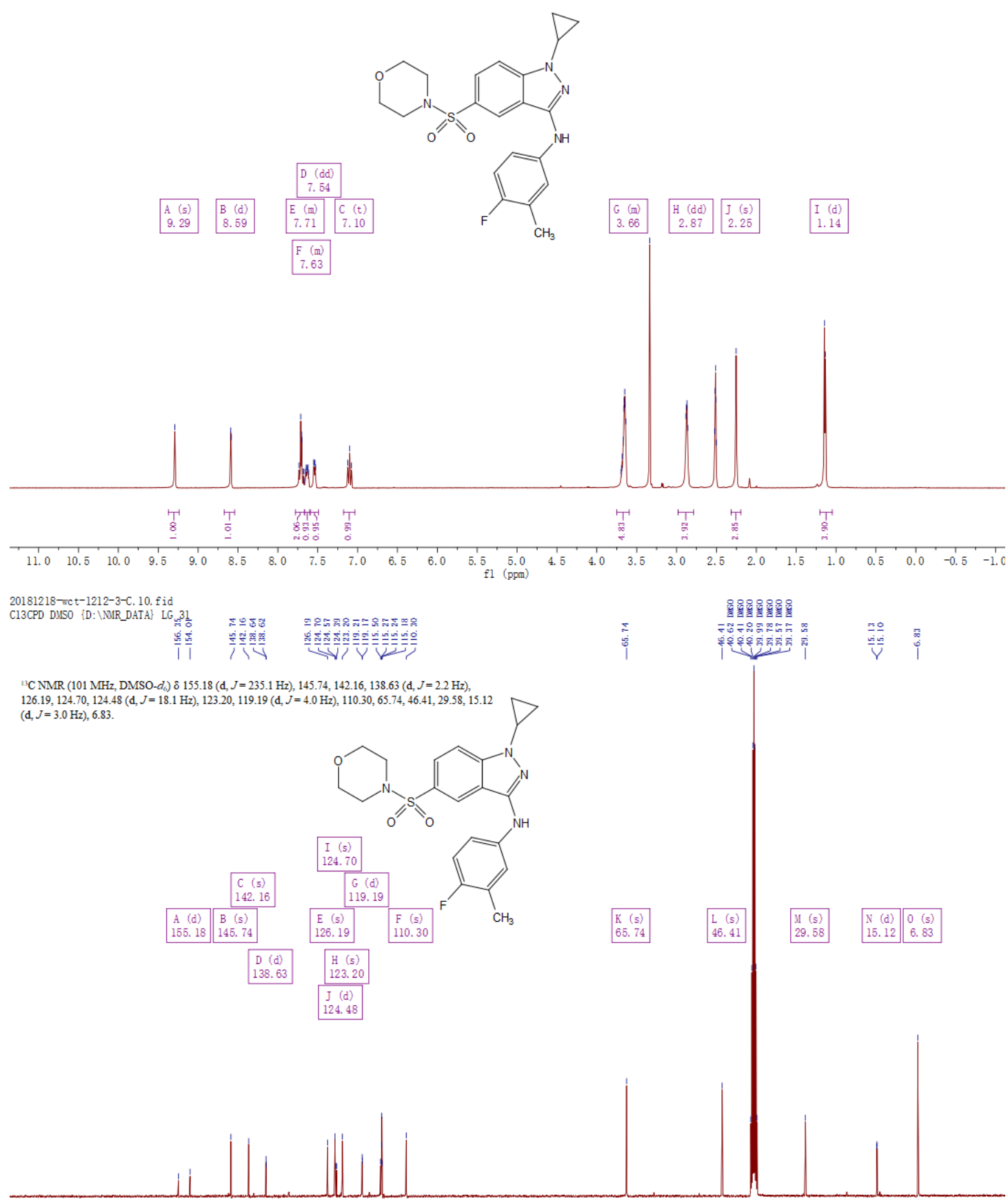

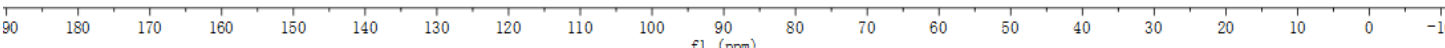




\section{Compound 41.}

20190717-wct-1419-1-H/10
PROTON DUSO \{D: INIR_DATA\} LG

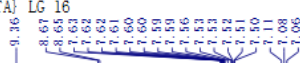

'H NMR ( $400 \mathrm{MHz}$, DMSO-d $\left.d_{6}\right) 89.36(\mathrm{~s}, 1 \mathrm{H}), 8.66(\mathrm{~d}, J=6.7 \mathrm{~Hz}, 1 \mathrm{H}$ ), 7.61 (ddd, $J=8.9,4.4,2.9 \mathrm{~Hz}$

$1 \mathrm{H}), 7.55(\mathrm{~d}, J=11.3 \mathrm{~Hz}, 1 \mathrm{H}), 7.52$ (dd, $J=6.8,2.8 \mathrm{~Hz}, 1 \mathrm{H}), 7.08(\mathrm{t}, J=9.2 \mathrm{~Hz}, 1 \mathrm{H}), 3.77-3.55(\mathrm{~m}$

$5 \mathrm{H}), 3.03$ (dd $J=5.7,3.5 \mathrm{~Hz}, 4 \mathrm{H}$, $22.4(\mathrm{~d}, J=1.8 \mathrm{~Hz}, 3 \mathrm{H}$ ), 1.11 (td, $J=52,4.6,3.1 \mathrm{~Hz}, 4 \mathrm{H}$ ).

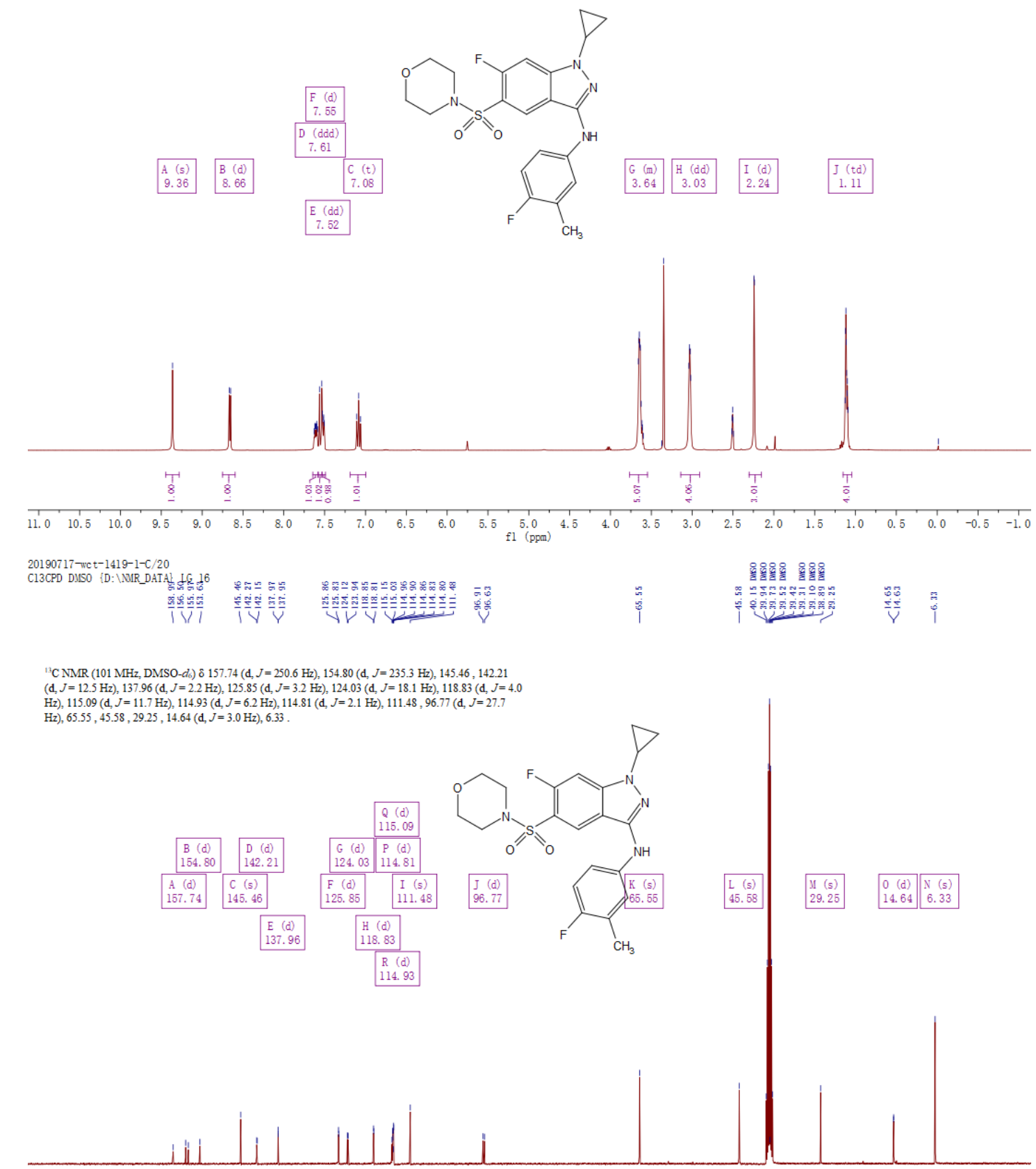

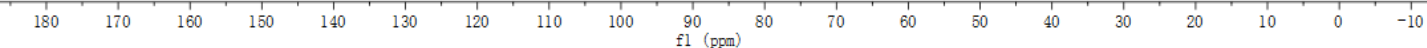




\section{Compound 42.}

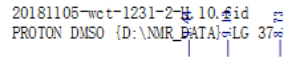
(1)

'H NMR (400 MHz, DMSO-d $\left.\mathrm{d}_{\mathrm{l}}\right) 89.44(\mathrm{~s}, 1 \mathrm{H}), 9.13(\mathrm{~s}, 1 \mathrm{H}), 8.73(\mathrm{~s}, 1 \mathrm{H}), 7.61$ (dt $\left., J=8.4,3.6 \mathrm{~Hz}, 1 \mathrm{H}\right)$, $7.52(\mathrm{dd}, J=6.8,2.8 \mathrm{~Hz}, 1 \mathrm{H}), 7.11(\mathrm{t}, J=9.2 \mathrm{~Hz}, 1 \mathrm{H}), 3.83(\mathrm{tt}, J=7.0,3.8 \mathrm{~Hz}, 1 \mathrm{H}), 3.64(\mathrm{t}, J=4.7 \mathrm{~Hz}$
$4 \mathrm{H}, 3.13(\mathrm{t} J=4.7 \mathrm{~Hz}, 4 \mathrm{H}), 2.26(\mathrm{~d}, J=1.8 \mathrm{~Hz}, 3 \mathrm{H}), 1.31-1.12(\mathrm{~m}, 4 \mathrm{H})$.

$$
\iiint \quad \int f \int
$$
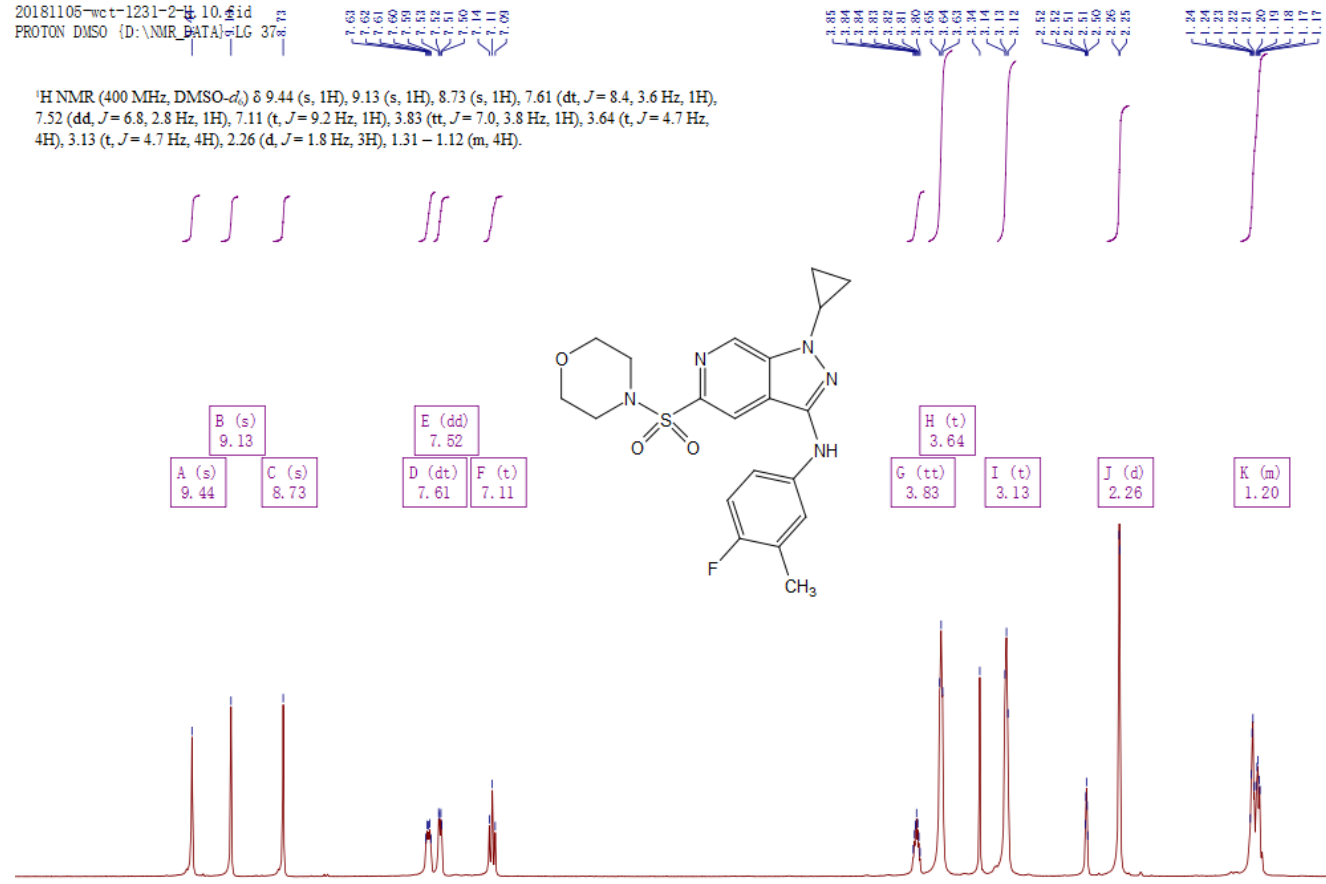

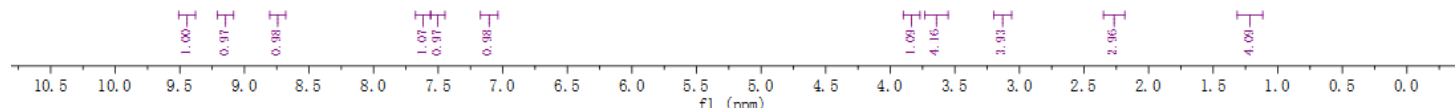

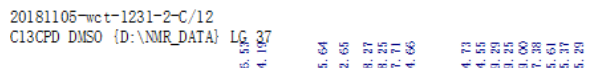

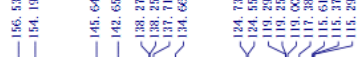

${ }^{13} \mathrm{C} \mathrm{NMR}\left(101 \mathrm{MHz}, \mathrm{DMSO}-d_{\mathrm{l}}\right) \delta 155.36$ (d, $\left.J=235.5 \mathrm{~Hz}\right), 145.64,142.65,138.26(\mathrm{~d}, J=2.2 \mathrm{~Hz})$

$137.71,134.66,124.64(\mathrm{~d}, J=18.0 \mathrm{~Hz}), 119.27(\mathrm{~d}, J=4.2 \mathrm{~Hz}), 119.00,117.38,115.49(\mathrm{~d}, J=23.4 \mathrm{~Hz})$, $115.29,66.07,46.85,30.20,15.11(\mathrm{~d}, J=2.9 \mathrm{~Hz}), 6.95$

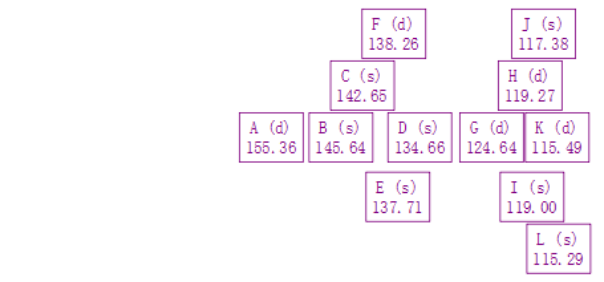<smiles>CNc1nn(C2CC2)c2cnc(S(=O)(=O)N3CCOCC3)cc12</smiles><smiles>Cc1cc(N)ccc1F</smiles>
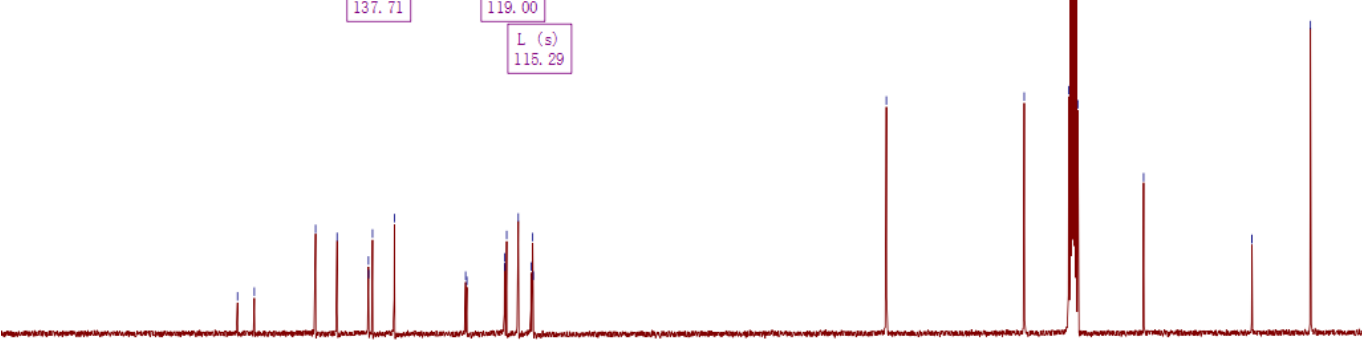


\section{Compound 43.}

\section{4-wct-1290-F-H. 10. fid}

i

in

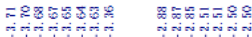

'H NMR (400 MHz, DMSO-d $\left.d_{\text {l }}\right) \delta 9.63(\mathrm{~s}, 1 \mathrm{H}), 8.57(\mathrm{~s}, 1 \mathrm{H}), 7.89$ (dd, $\left.J=13.8,7.1 \mathrm{~Hz}, 1 \mathrm{H}\right), 7.72(\mathrm{q}, J=$

$8.9 \mathrm{~Hz}, 2 \mathrm{H}), 7.45-7.33(\mathrm{~m}, 2 \mathrm{H}), 3.66(\mathrm{dt}, J=15.6,5.0 \mathrm{~Hz}, 5 \mathrm{H}), 2.87(\mathrm{t}, J=4.5 \mathrm{~Hz}, 4 \mathrm{H}), 1.15(\mathrm{~d}, J=5$.

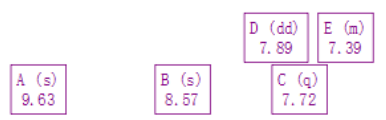<smiles></smiles>

\begin{tabular}{|c|c|}
\hline F (dt) \\
3.66
\end{tabular}$\quad \begin{aligned} & G(t) \\
& 2.87\end{aligned}$

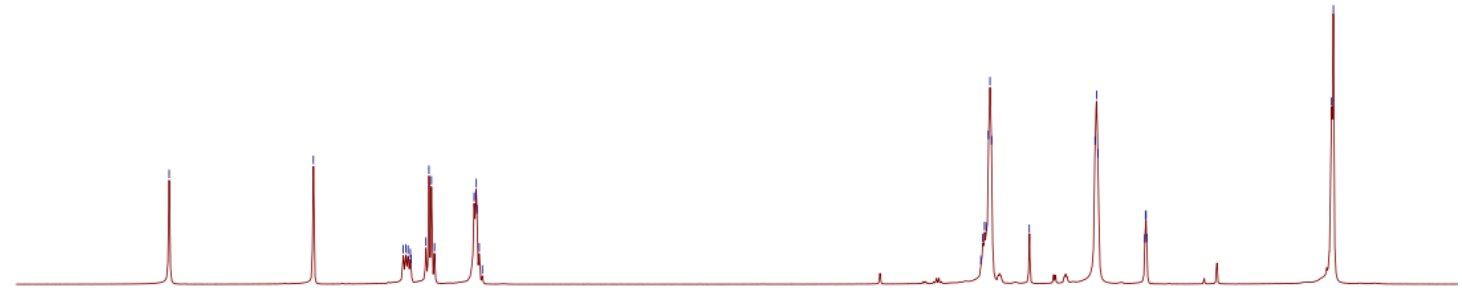

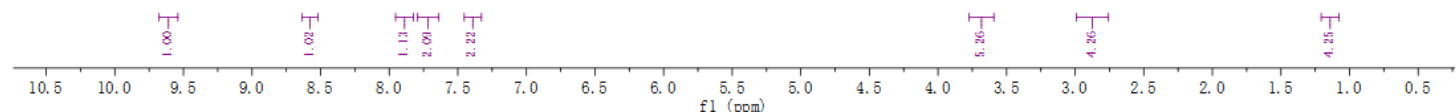

20190104-wct-1290-2-C. 10, fid

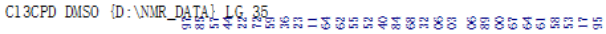

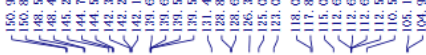

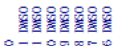

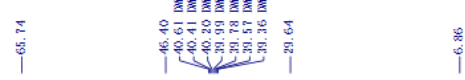

${ }^{13} \mathrm{C} \mathrm{NMR}\left(101 \mathrm{MHz}\right.$, DMSO- $d_{\text {) }} \delta 149.71$ (dd, $J=241.7,12.9 \mathrm{~Hz}$ ), $145.22,143.48$ (dd, $J=237.1,12.9$

$\mathrm{Hz}), 142.11,139.58$ (dd, $J=9.6,2.2 \mathrm{~Hz}), 126.32,125.06,123.03,117.97$ (d, $J=17.8 \mathrm{~Hz}), 115.00,112.62$ (dd, $J=5.6,2.9 \mathrm{~Hz}), 110.53,105.06(\mathrm{~d}, J=22.1 \mathrm{~Hz}), 65.74,46.40,29.64,686$.

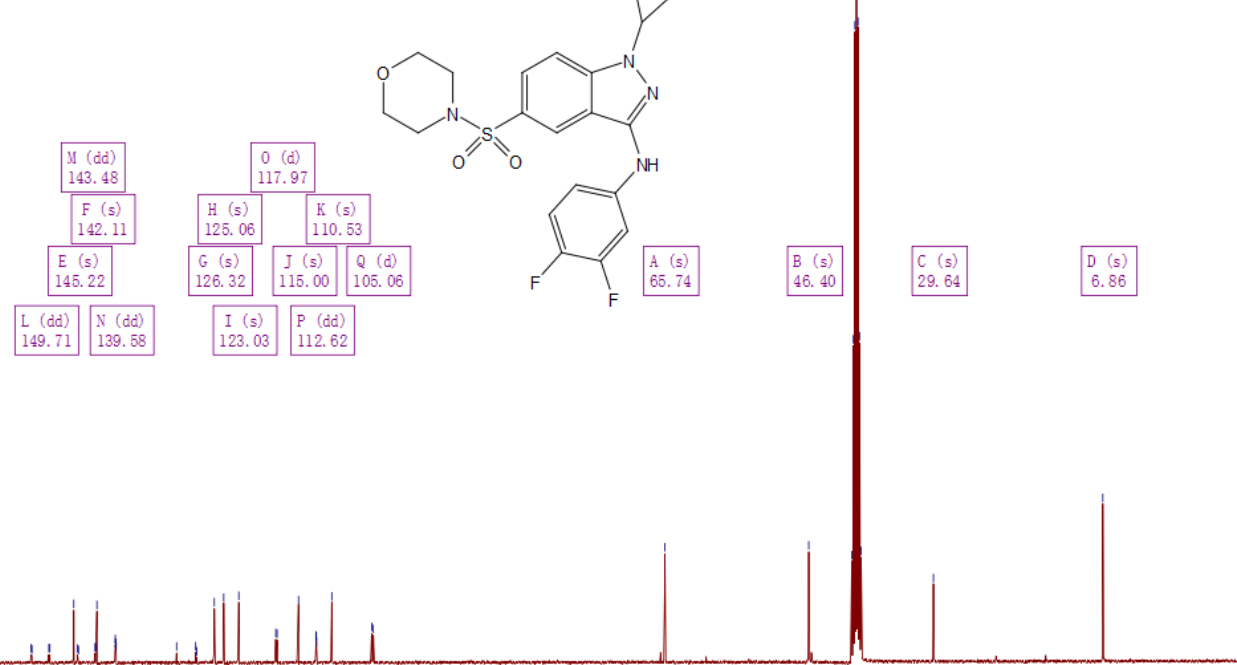




\section{Compound 44.}

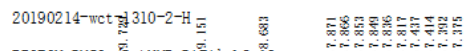

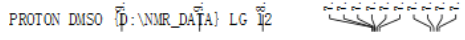

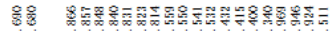

ن

'H NMR (400 MHz, DMSO) $\delta 9.73(\mathrm{~s}, 1 \mathrm{H}), 9.15$ (s, 1H), 8.68 (s, $1 \mathrm{H}), 7.92-7.76(\mathrm{~m}, 1 \mathrm{H}), 7.40$ (dd, $J=$ $3.36(\mathrm{~m}, 2 \mathrm{H}), 2.95(\mathrm{t} J=9.0 \mathrm{~Hz}, 2 \mathrm{H}), 1.44-166(\mathrm{~m}, 2 \mathrm{~m}, 1 \mathrm{H}), 3.55(\mathrm{dd}, J=7.4,3.7 \mathrm{~Hz}, 1 \mathrm{H}), 3.49$

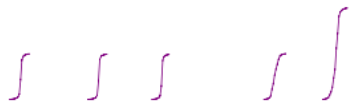<smiles>CCCCC</smiles><smiles>Cc1nc2cc(S(=O)(=O)N3CCC(O)CC3)ncc2cc1N(C1CC1)C1CC1</smiles>

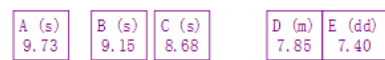

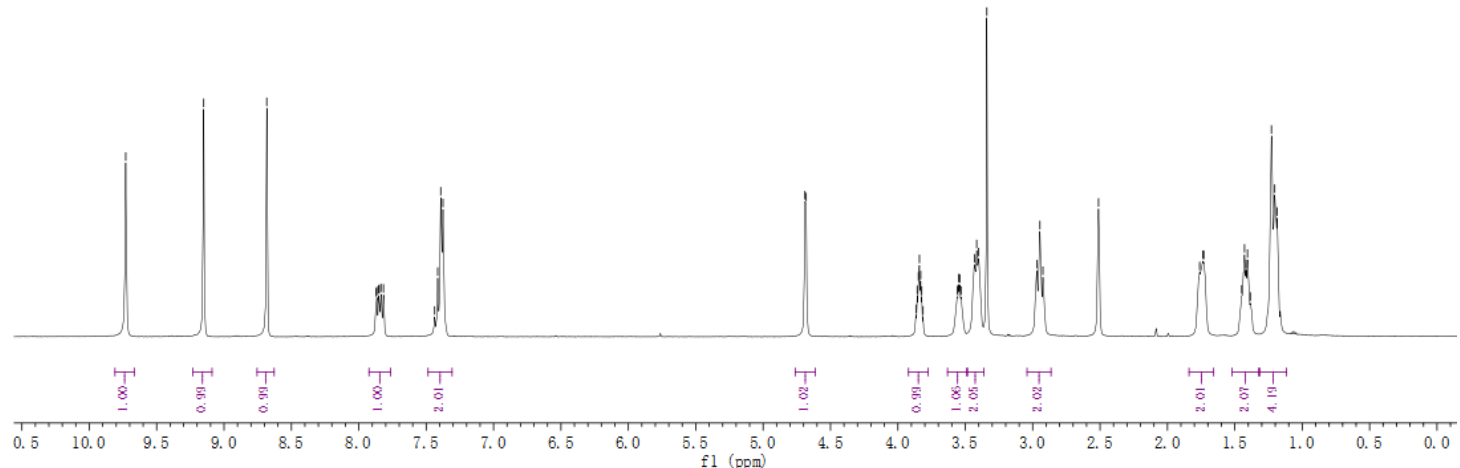

20190222-wct-1310-2-H/10

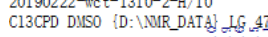

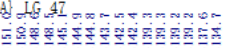

等

${ }_{13}^{13} \mathrm{C} \mathrm{NMR}(101 \mathrm{MHz}$, DMSO-d ) $\delta 149.72$ (dd, $J=242.1,13.2 \mathrm{~Hz}$ ), 145.07, $143.71,143.66$ (dd, $J=$

$237.6,12.8 \mathrm{~Hz}), 139.23(\mathrm{dd}, J=9.3,2.2 \mathrm{~Hz}), 137.58,134.69,118.87,118.06(\mathrm{~d}, J=18.0 \mathrm{~Hz}), 116.66$

$112.71(\mathrm{dd}, J=5.2,2.7 \mathrm{~Hz}), 105.14(\mathrm{~d}, J=22.0 \mathrm{~Hz}), 64.78,44.17,33.77,30.25,6.95$.

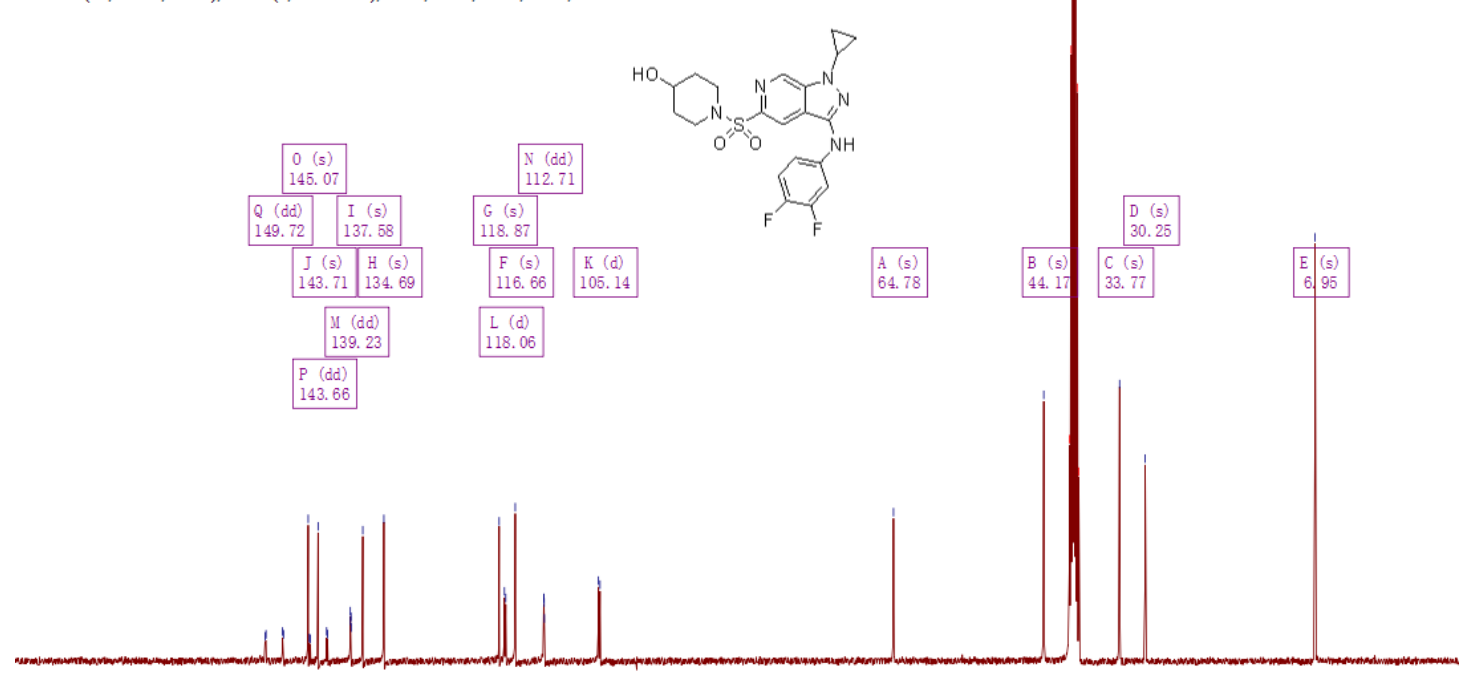

$\begin{array}{lllllllll}1 & 1 & 1 & 1 & 1 & 1 & 1 & 1 & 1 \\ 70 & 50 & 40 & 30 & 20 & 10 & 0 & -10\end{array}$




\section{Compound 45.}

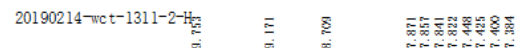

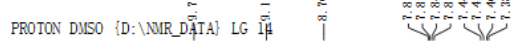

'H NMR (400 MHz, DMSO) $89.75(\mathrm{~s}, 1 \mathrm{H}), 9.17(\mathrm{~s}, 1 \mathrm{H}), 8.71(\mathrm{~s}, 1 \mathrm{H}), 7.85$ (dd, $J=12.8,6.6 \mathrm{~Hz}, 1 \mathrm{H}$ ), $7.41(\mathrm{dd}, J=17.8,7.7 \mathrm{~Hz}, 2 \mathrm{H}), 3.85(\mathrm{~d}, J=3.7 \mathrm{~Hz}, 1 \mathrm{H}), 3.64(\mathrm{~s}, 4 \mathrm{H}), 3.13(\mathrm{~s}, 4 \mathrm{H}), 1.34-1.08(\mathrm{~m}, 4 \mathrm{H})$
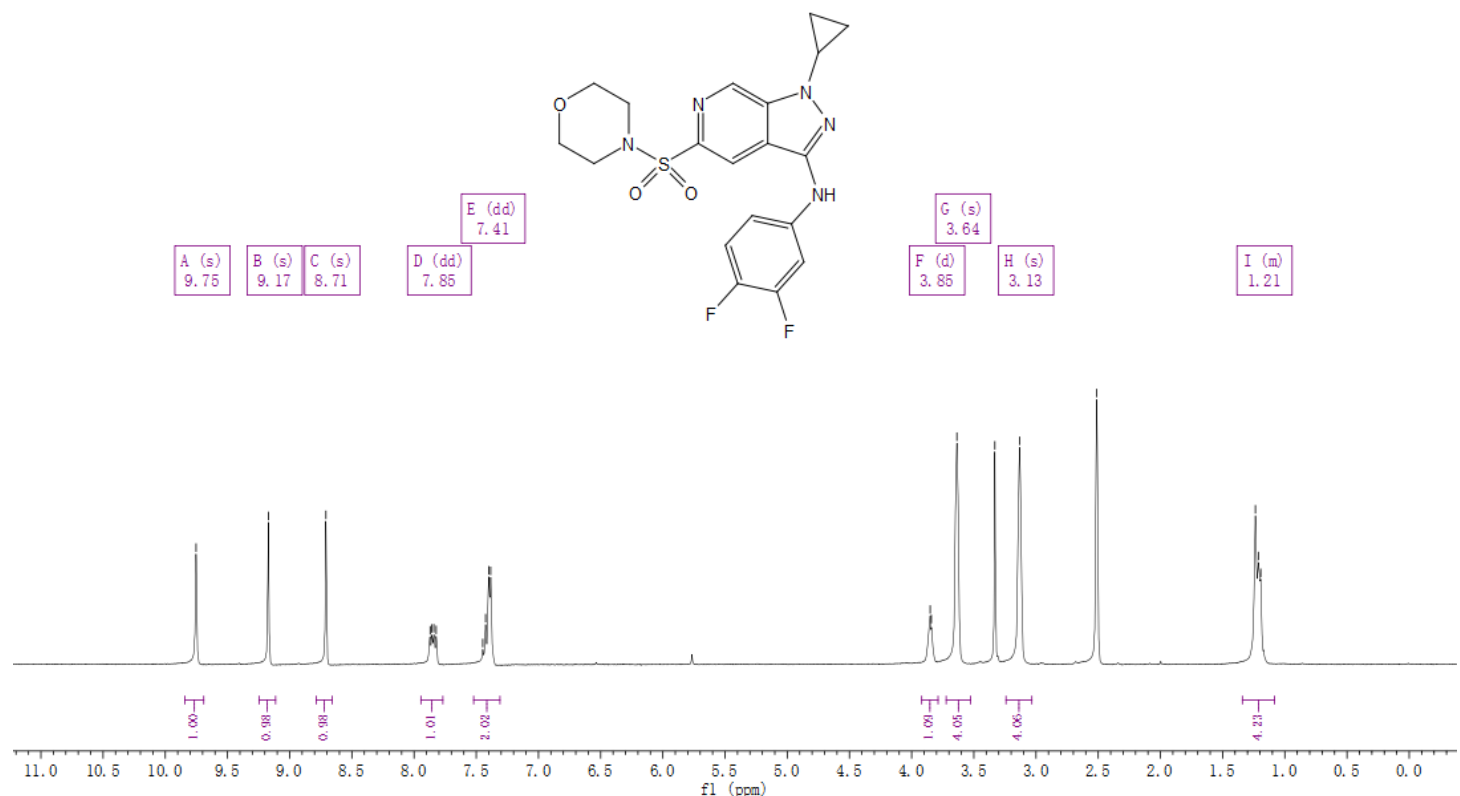

$20190226-w c t-1311-2-\mathrm{C} / 10$

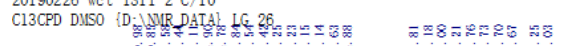

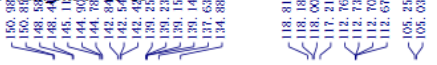

${ }^{13} \mathrm{C} \mathrm{NMR}\left(101 \mathrm{MHz}, \mathrm{DMSO}-d_{\mathrm{l}}\right.$ ) $\delta 149.71$ (dd, $J=242.0,13.3 \mathrm{~Hz}$ ), $145.11,143.66$ (dd, $J=237.7,12.8$

$\mathrm{Hz}), 142.84,139.19$ (dd, $J=9.5,2.1 \mathrm{~Hz}), 137.63,134.88,118.81,118.09(\mathrm{~d}, J=17.8 \mathrm{~Hz}), 117.21$,
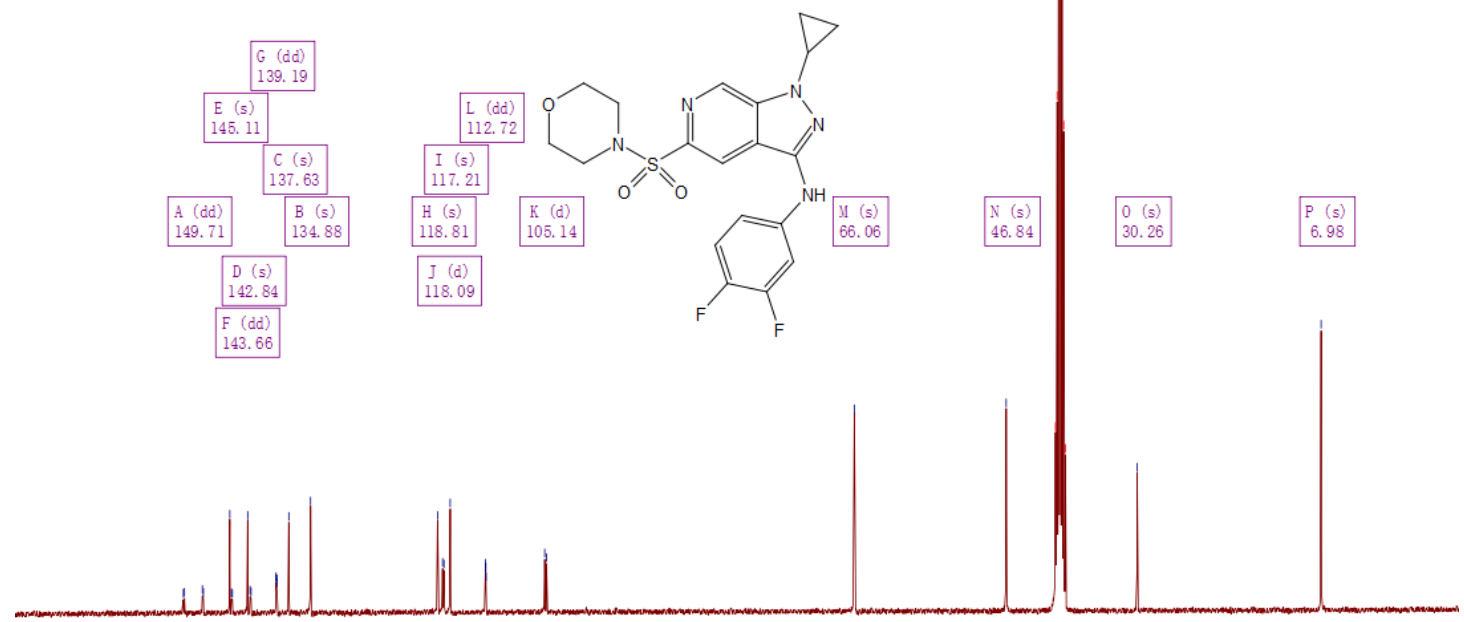

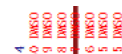
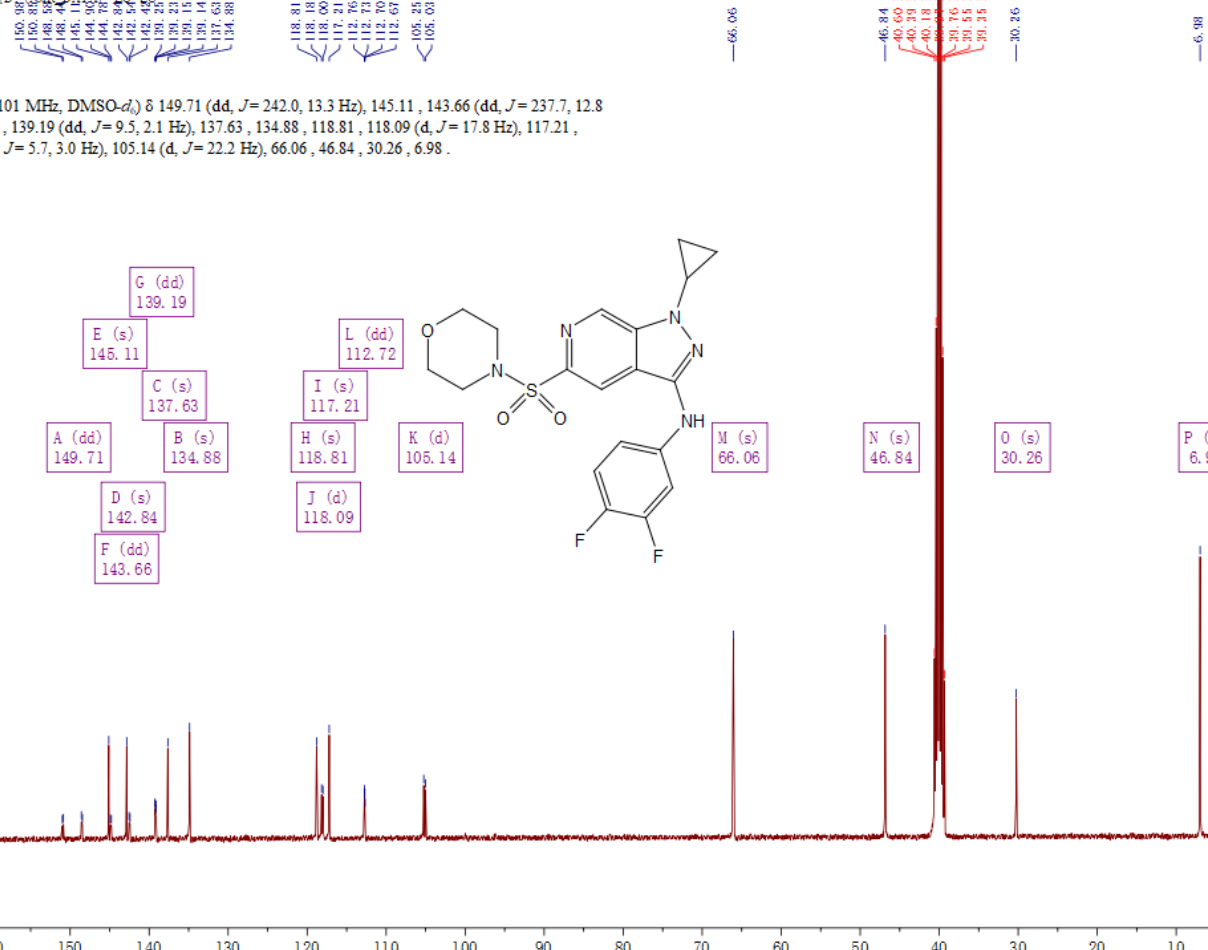

$120 \quad 110$

100

$1 \stackrel{80}{(\mathrm{ppm})}$ 


\section{Compound 46.}

20181229-wct-1286-1-H. 10, fid
PROTON DUSO \{D: \NMR_DATA\} LG 3 i

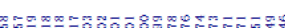
in 整这

H NMR $\left(400 \mathrm{MHz}\right.$, DMSO- $\left.d_{\mathrm{d}}\right) \delta 9.77(\mathrm{~s}, 1 \mathrm{H}), 8.58(\mathrm{~d}, J=1.5 \mathrm{~Hz}, 1 \mathrm{H}), 8.18$ (dd, $\left.J=6.3,2.8 \mathrm{~Hz}, 1 \mathrm{H}\right)$, 8.01 (dt, $J=8.9,3.6 \mathrm{~Hz}, 1 \mathrm{H}), 7.77(\mathrm{~d}, J=8.9 \mathrm{~Hz}, 1 \mathrm{H}), 7.72(\mathrm{dd}, J=8.8,1.7 \mathrm{~Hz}, 1 \mathrm{H}), 7.49(\mathrm{t}, J=9.8 \mathrm{~Hz}$<smiles>CC=C(C=C1C=CC=C1)CC</smiles>
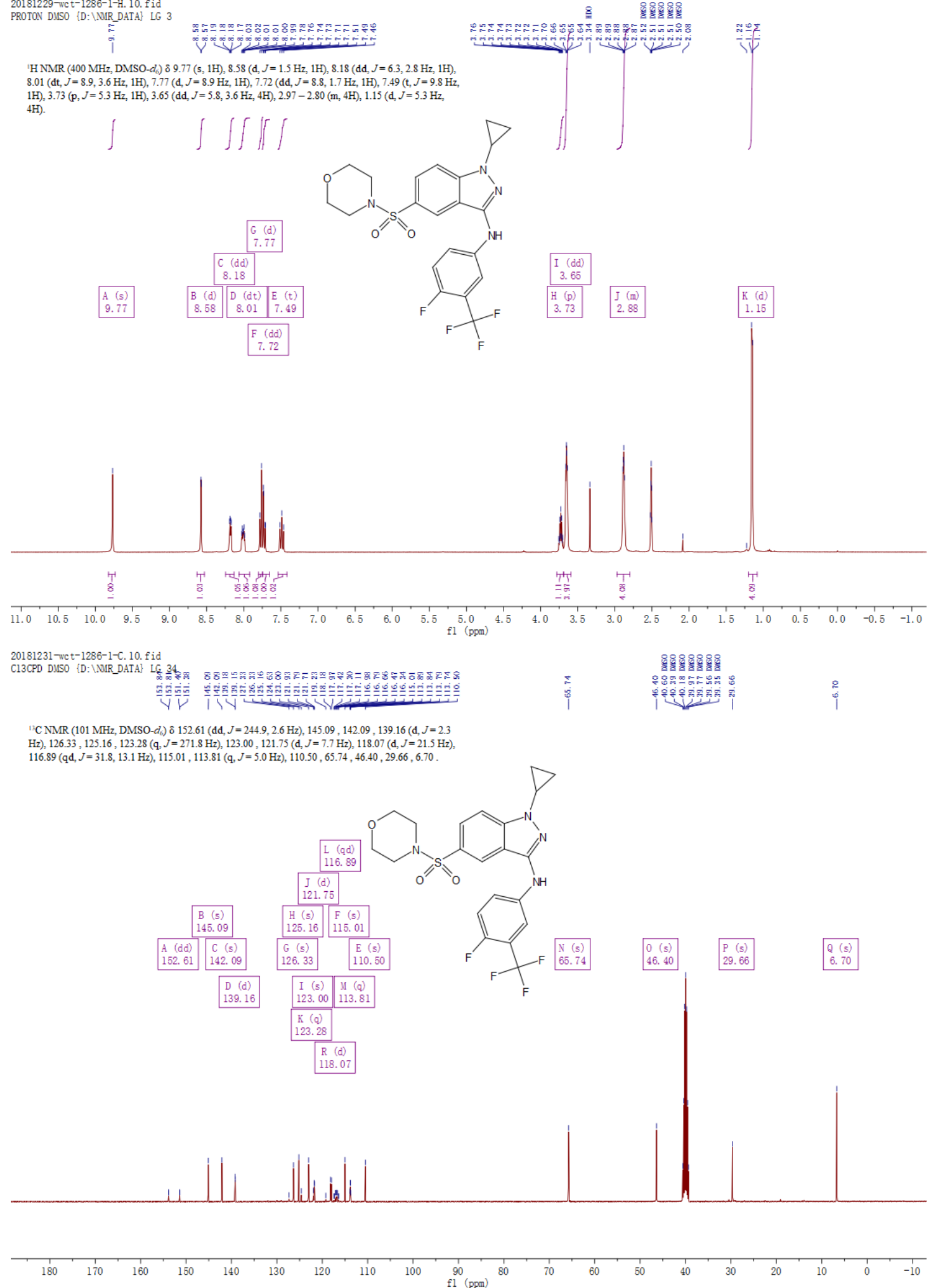


\section{Compound 47.}

20190115-wet-1297-3-H. 10, fid
PROTON DUSO \{D:LNIR__ATAA\} LG 12

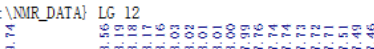

H NMR (400 MHz, DMSO- $\left.d_{6}\right) \delta 9.74(\mathrm{~s}, 1 \mathrm{H}), 8.56(\mathrm{~s}, 1 \mathrm{H}), 8.17$ (dd, $J=6.3,2.8 \mathrm{~Hz}, 1 \mathrm{H}$ ), 8.01 (dt, $J=$

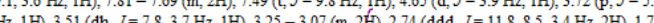

(ddt $J=10.6,69,35 . \mathrm{Hz}, 2 \mathrm{~Hz}, 1.45$ (dtd $J=119,79,3,5 \mathrm{~Hz}, 2 \mathrm{H}), 115(\mathrm{~d}, J=53 \mathrm{Hzz}, 4 \mathrm{H}$ )

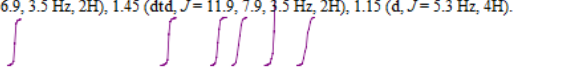

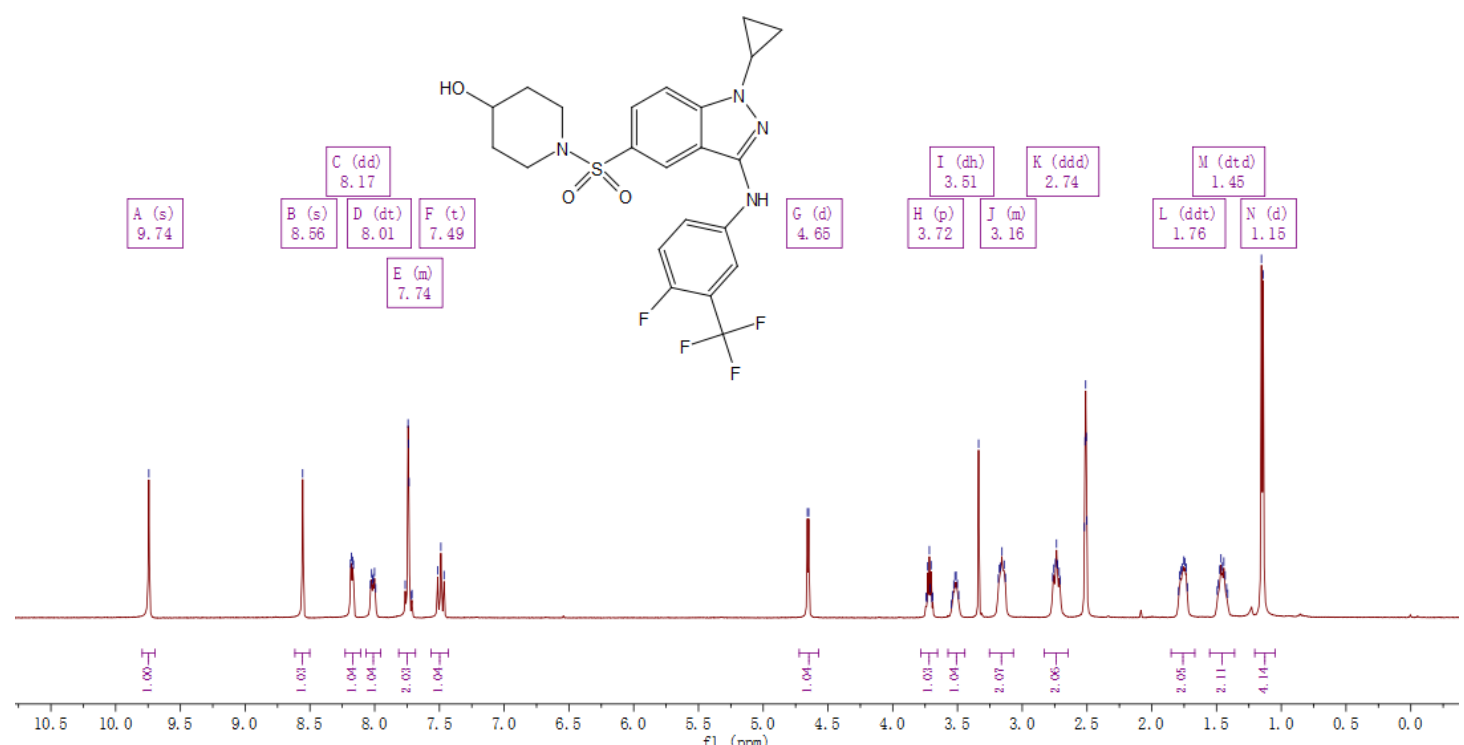

20190115-wct-1297-3-C. 10. fid
C13CPD DISO \{D: \NIR_DATA\} LG J3

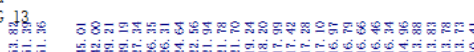

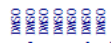

VY

${ }^{12} \mathrm{CNMR}\left(101 \mathrm{MHz}, \mathrm{DMSO}-d_{\mathrm{s}}\right) \delta 154.28-150.91(\mathrm{~m}), 145.01,142.00,139.20(\mathrm{~d}, J=2.2 \mathrm{~Hz}), 126.33$ $(\mathrm{d}, J=3.3 \mathrm{~Hz}), 123.29(\mathrm{q}, J=271.8 \mathrm{~Hz}), 122.56,121.74(\mathrm{~d}, J=7.5 \mathrm{~Hz}), 118.20,117.99,116.88$ (qd, $J=$

$32.0,13.0 \mathrm{~Hz}), 114.96,113.81(\mathrm{q}, J=5.3,4.8 \mathrm{~Hz}), 110.41,64.21,43.69,33.36,29.66,6.71$

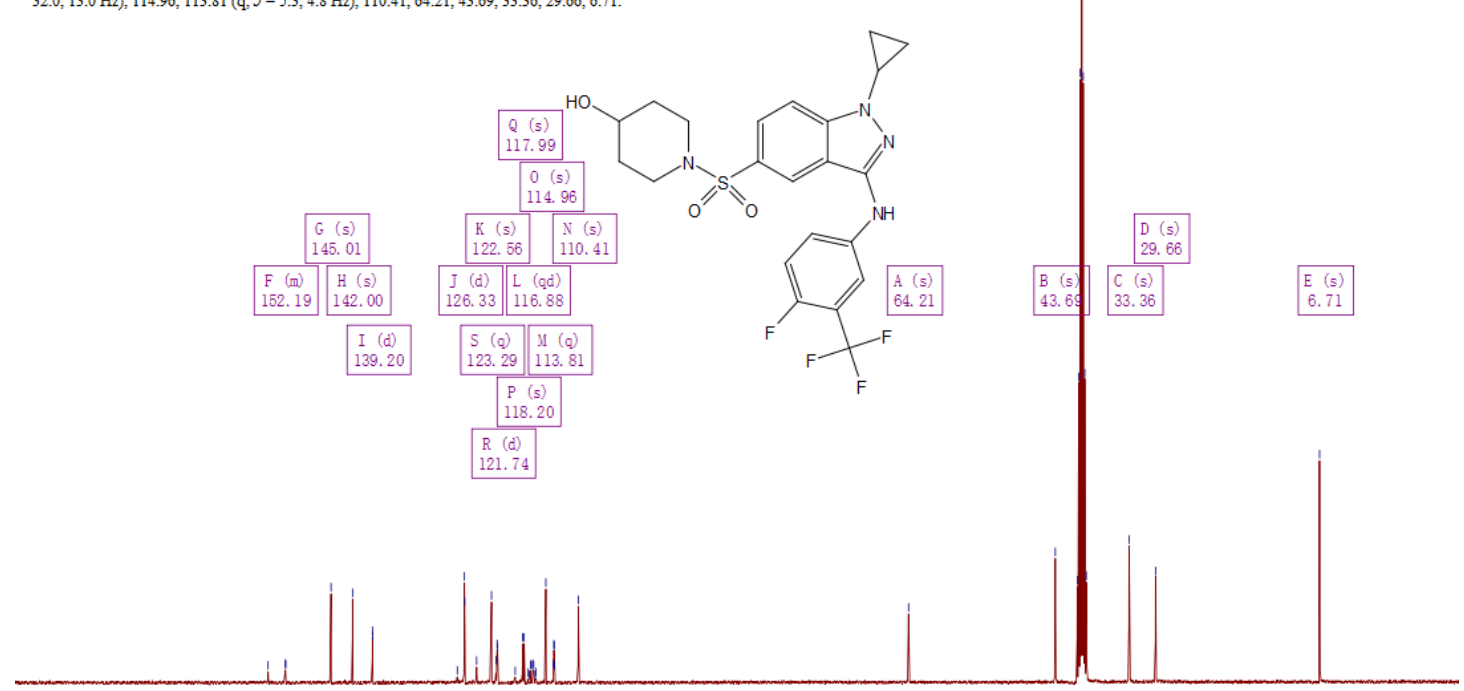

180

$170 \quad 160$

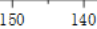

$130 \quad 120$

$110 \quad 100$

fl 90

$70 \quad 60$

30

(20 


\section{Compound 48.}

20190115-wct-1298-1-H. 10, fid
PROTON DUSO \{D: \NIR_DATA\} LG 13

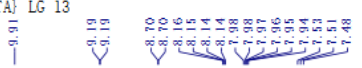

H NMR ( $400 \mathrm{MHz}$, DMSO- $\left.d_{6}\right) \delta 9.91(\mathrm{~s}, 1 \mathrm{H}), 9.19(\mathrm{~d}, J=1.1 \mathrm{~Hz}, 1 \mathrm{H}), 8.70(\mathrm{~d}, J=1.1 \mathrm{~Hz}, 1 \mathrm{H}), 8.15$ (dd, $J=6.2,2.9 \mathrm{~Hz}, 1 \mathrm{H}), 7.96$ (dt $, J=9.0,3.6 \mathrm{~Hz}, 1 \mathrm{H}), 7.51(\mathrm{t}, J=9.8 \mathrm{~Hz}, 1 \mathrm{H}), 3.94-3.83(\mathrm{~m}, 1 \mathrm{H}), 3.64$ (dd, $J=5.9,3.5 \mathrm{~Hz}, 4 \mathrm{H}), 3.25-3.00(\mathrm{~m}, 4 \mathrm{H}), 1.21$ (ddt. $J=12.7,9.3,5.0 \mathrm{~Hz}, 4 \mathrm{H})$.

$$
\iiint \iiint
$$

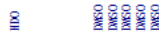

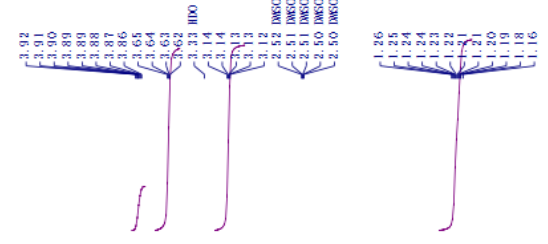

\begin{tabular}{|l|l|l|l|l|l|}
\hline $\begin{array}{l}\text { (s) } \\
9.91\end{array}$ & $\begin{array}{l}\text { B (d) } \\
9.19\end{array}$ & $\begin{array}{c}\text { C (d) } \\
8.70\end{array}$ & $\begin{array}{c}\text { F (dd) } \\
8.15\end{array}$ & $\begin{array}{c}\text { D (t) } \\
7.51\end{array}$ \\
\hline
\end{tabular}<smiles>O=S(=O)(c1ccc2c(c1)c(Nc1ccc(F)c(C(F)(F)F)c1)nn2C1CC1)N1CCOCC1</smiles>
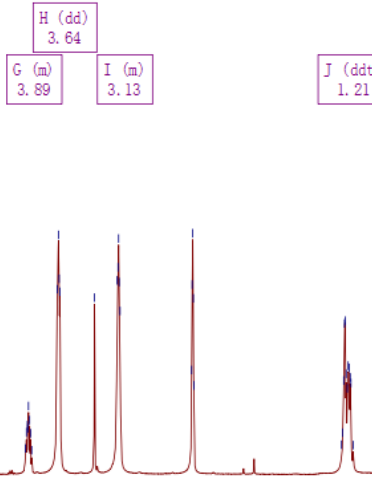

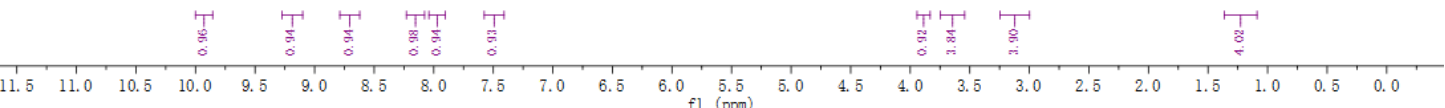

20190115-wct-1298-1-H_2. 10.fid
C13CPD DISO \{D: LNMR_DATA\} LG 14

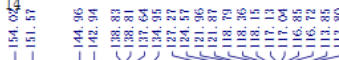

${ }^{13} \mathrm{C} \mathrm{NMR}$ (101 MHz, DMSO- $\left.d_{\text {) }}\right) \delta 152.79$ (d, $\left.J=245.9 \mathrm{~Hz}\right), 144.96,142.94,138.82$ (d, $\left.J=2.5 \mathrm{~Hz}\right)$, $137.64,134.95,128.87-123.58(\mathrm{~m}), 121.92(\mathrm{~d}, J=8.5 \mathrm{~Hz}), 118.79,118.26$ (d, $J=21.3 \mathrm{~Hz}), 117.13$, $137.64,134.95,128.87-123.58(\mathrm{~m}), 121.92(\mathrm{~d}, J=8.5 \mathrm{~Hz}), 118.79$,
$117.25-116.64(\mathrm{~m}), 113.83(\mathrm{~d}, J=5.2 \mathrm{~Hz}), 66.06,46.84,30.31,6.85$
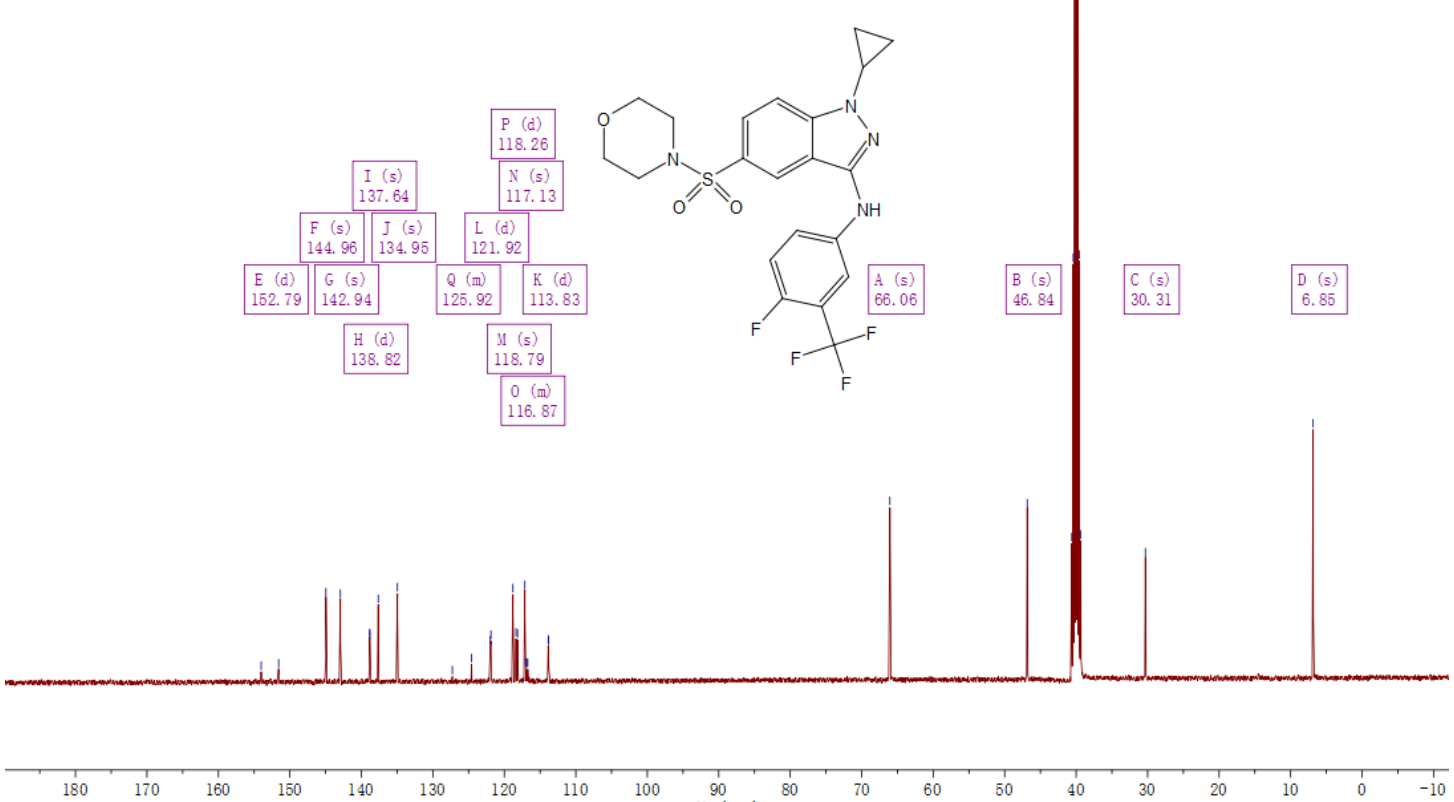

$100 \quad 90$

$80 \quad 70$

60

50

$40 \quad 30$

$10+1,-10$ 


\section{Compound 49.}

20190108-wct-1294-1-H. 10, fid
PROTON DUSO \{D: $:$ NNR_DATA\} LG 23

233000

'H NMR (400 MHz, DMSO- $\left.d_{s}\right) \delta 9.26(\mathrm{~s}, 1 \mathrm{H}), 8.55(\mathrm{~s}, 1 \mathrm{H}), 7.70(\mathrm{~s}, 2 \mathrm{H}), 7.62-7.52$ (m, $\left.1 \mathrm{H}\right), 7.31$ (dd,

$J=6.8,2.8 \mathrm{~Hz}, 1 \mathrm{H}), 7.09(\mathrm{t}, J=9.5 \mathrm{~Hz}, 1 \mathrm{H}), 4.65(\mathrm{~d}, J=3.8 \mathrm{~Hz}, 1 \mathrm{H}), 3.75-3.61(\mathrm{~m}, 1 \mathrm{H}), 3.51(\mathrm{tt}, J=$

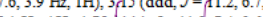

$5.1 \mathrm{~Hz}, 1 \mathrm{H}$ ), 1.75 (ddd, $J=11.3,7.4,3.6 \mathrm{~Hz}, 2 \mathrm{H}$ ), 1.45 (dtd $J=12.3,8.2,3.6 \mathrm{~Hz}, 2 \mathrm{H}$ ), 1.12 (d, $J=2.3$

$\mathrm{Hz}, 4 \mathrm{H}), 1.03(\mathrm{dt}, J=8.7,3.2 \mathrm{~Hz}, 2 \mathrm{H}), 0.78-0.66(\mathrm{~m}, 2 \mathrm{H})$
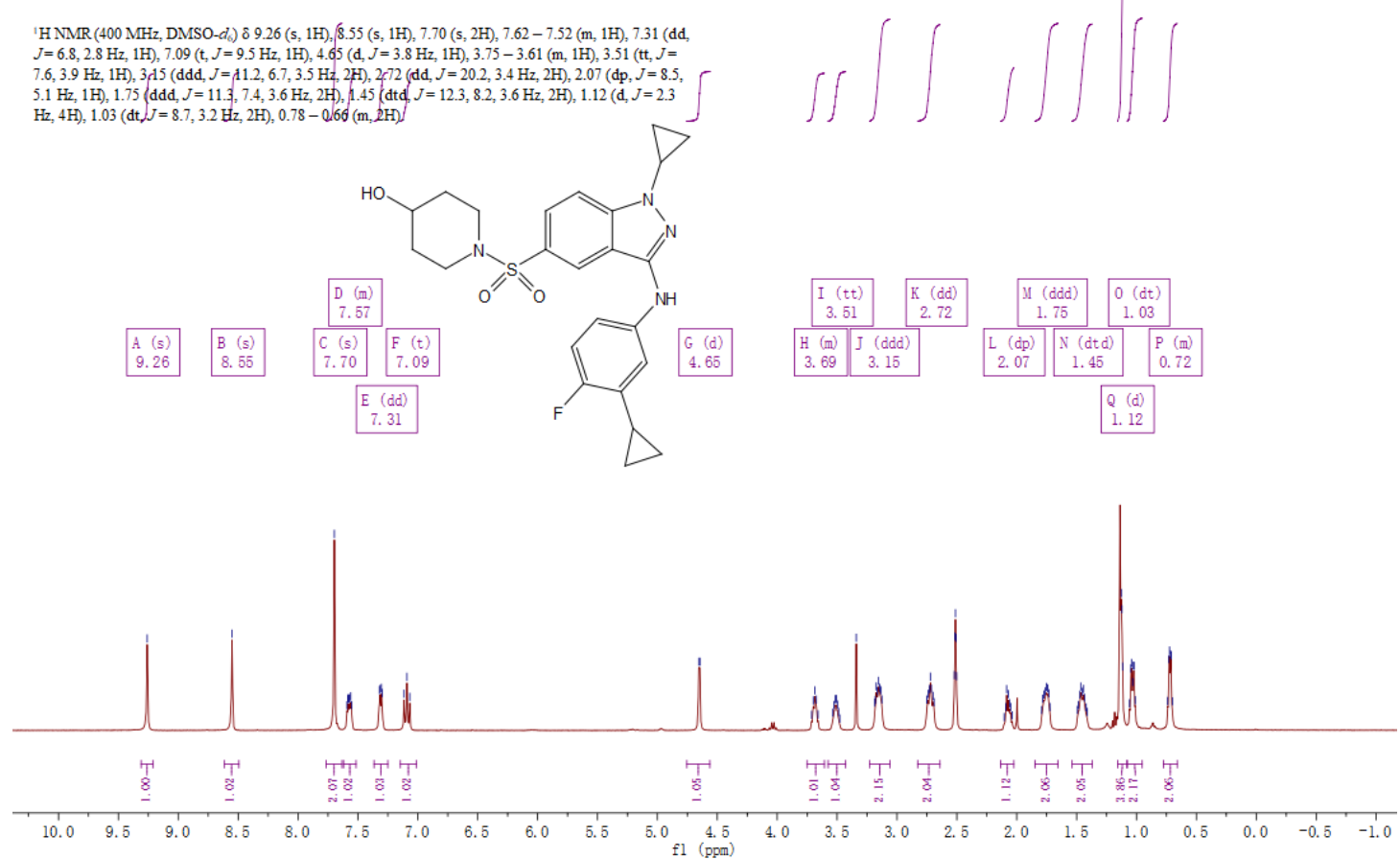

20190111-wct-1294-1-C. 10. fid

C13CPD DUSO $\{\mathrm{D}: \backslash$ NYR D. 10 . fid

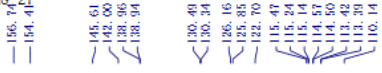

${ }^{12} \mathrm{C} \mathrm{NMR}\left(101 \mathrm{MHz}, \mathrm{DMSO}-d_{\text {) }}\right) 8155.58$ (d, $\left.J=235.3 \mathrm{~Hz}\right), 145.61,142.00,138.95$ (d, $\left.J=2.0 \mathrm{~Hz}\right)$ $130.42(\mathrm{~d}, J=15.3 \mathrm{~Hz}), 126.16,125.85,122.70,115.35(\mathrm{~d}, J=23.1 \mathrm{~Hz}), 115.14,114.54(\mathrm{~d}, J=7.5 \mathrm{~Hz})$ $113.41(\mathrm{~d}, J=3.4 \mathrm{~Hz}), 110.14,64.24,43.71,33.36,29.59,8.94(\mathrm{~d}, J=4.7 \mathrm{~Hz}), 8.53,6.67$.

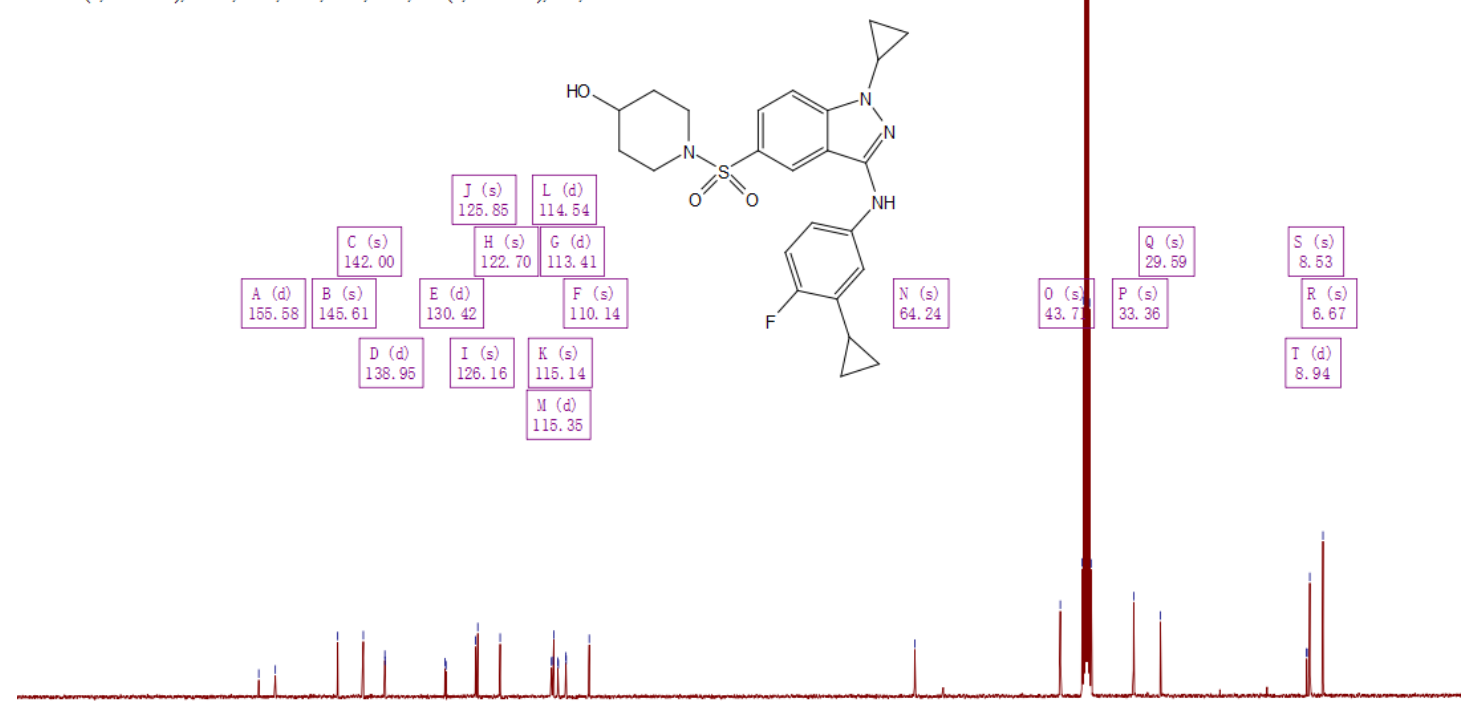

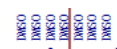

|

$\begin{array}{llllllllllllllllllllllllll}1 & 180 & 170 & 160 & 150 & 140 & 130 & 120 & 110 & 100 & 90 & 80 & 70 & 60 & 50 & 40 & 30 & 20 & 10 & 0 & -10\end{array}$ 


\section{Compound 50.}

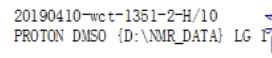

'H NMR ( $400 \mathrm{MHz}$, DMSO- $\left.d_{k}\right) \delta 9.44(\mathrm{~s}, 1 \mathrm{H}), 8.68(\mathrm{~s}, 1 \mathrm{H}), 7.89(\mathrm{~d}, J=8.8 \mathrm{~Hz}, 1 \mathrm{H}), 7.78(\mathrm{~d}, J=9.0$

$\mathrm{Hz}, 1 \mathrm{H}), 7.65(\mathrm{dt}, J=8.2,3.6 \mathrm{~Hz}, 1 \mathrm{H}), 7.59(\mathrm{~d}, J=6.9 \mathrm{~Hz}, 1 \mathrm{H}), 7.11(\mathrm{t}, J=9.2 \mathrm{~Hz}, 1 \mathrm{H}), 5.39(\mathrm{q}, J=9.0$

$\mathrm{Hz}, 2 \mathrm{H}), 3.66(\mathrm{t}, J=4.6 \mathrm{~Hz}, 4 \mathrm{H}), 2.90(\mathrm{t}, J=4.6 \mathrm{~Hz}, 4 \mathrm{H}), 2.26(\mathrm{~s}, 3 \mathrm{H})$
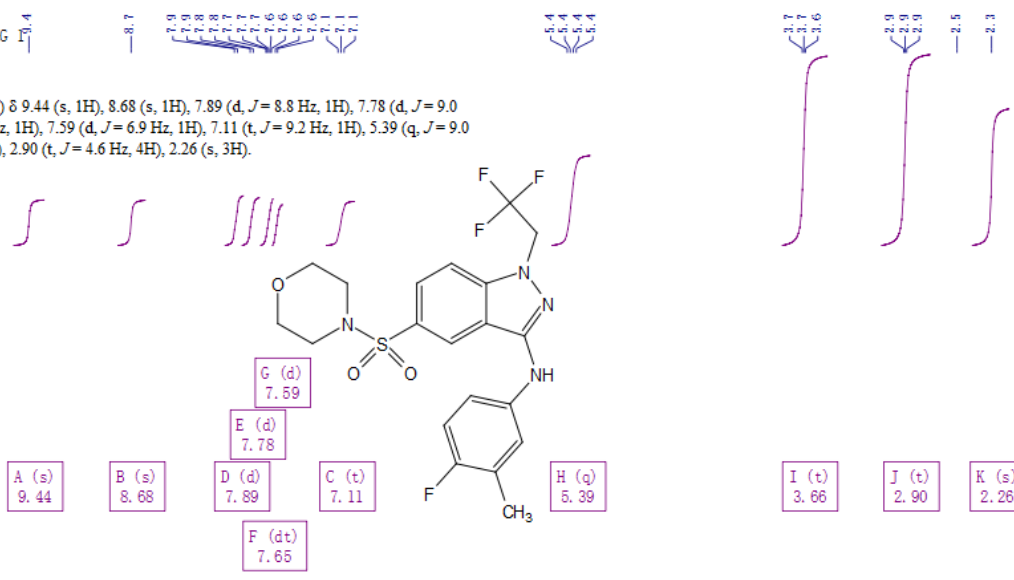

\section{\begin{tabular}{|l|l|l|}
\hline $\begin{array}{l}I(t) \\
3.66\end{array}$ & $\begin{array}{ll}J(t) \\
2.90\end{array}$ & $\begin{array}{l}K(s) \\
2.26\end{array}$ \\
\hline
\end{tabular}}

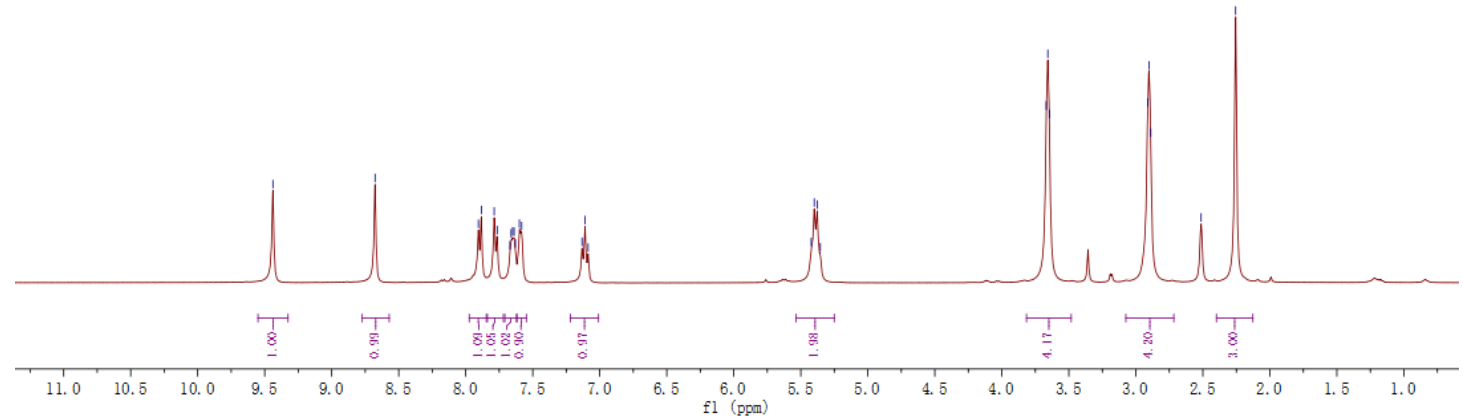

$20190416-w c t-1351-2-C / 10$

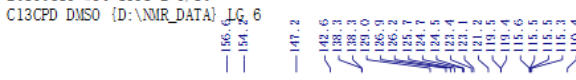

${ }^{13} \mathrm{CNMR}\left(101 \mathrm{MHz}, \mathrm{DMSO}-d_{\mathrm{s}}\right) \delta 155.40(\mathrm{~d}, J=235.5 \mathrm{~Hz}), 147.17,142.58,138.31(\mathrm{~d}, J=22 \mathrm{~Hz})$,

$126.90,126.25(\mathrm{q}, J=281.5 \mathrm{~Hz}), 125.74,124.57(\mathrm{~d}, J=18.0 \mathrm{~Hz}), 123.15,119.46(\mathrm{~d}, J=4.0 \mathrm{~Hz}), 115.60$

115.52, $115.37(\mathrm{~d}, J=16.7 \mathrm{~Hz}), 110.44,65.75,49.24(\mathrm{q}, J=32.8 \mathrm{~Hz}), 46.43,15.09(\mathrm{~d}, J=3.0 \mathrm{~Hz})$.

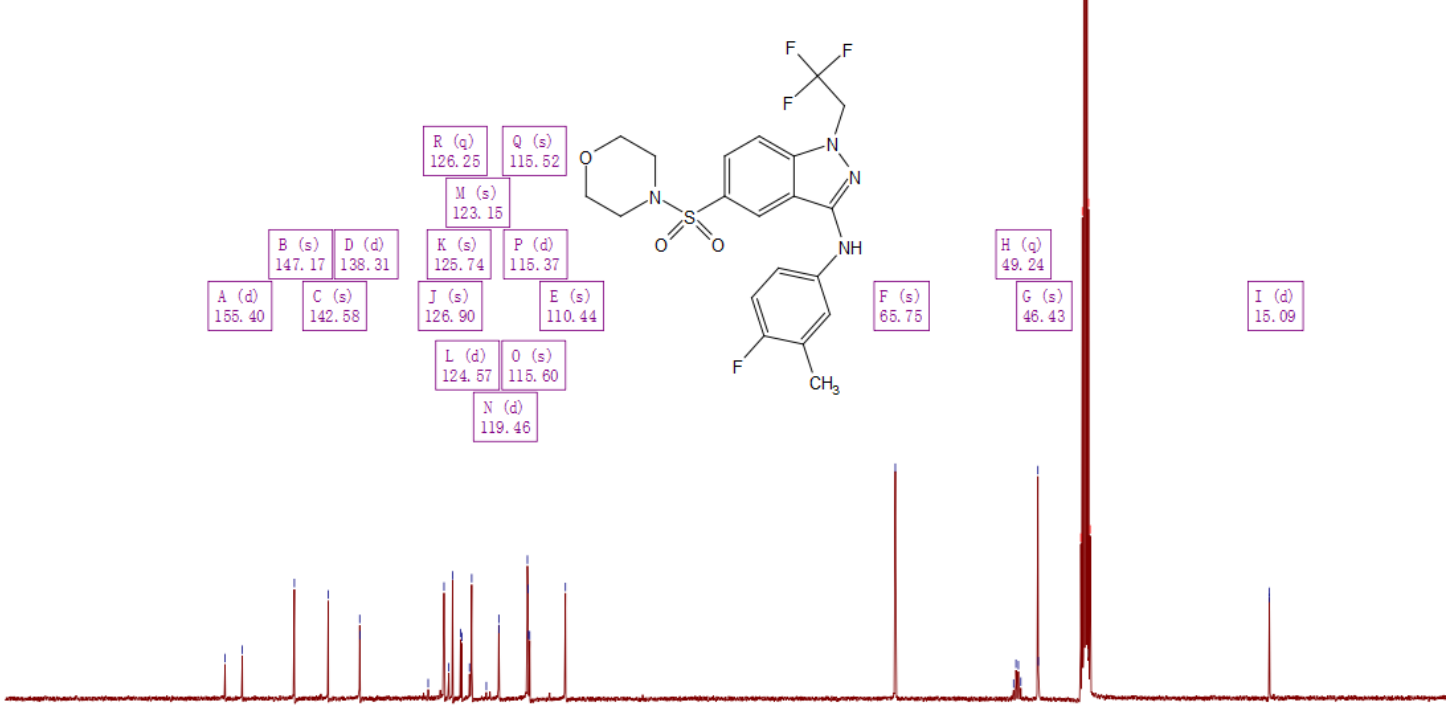




\section{Compound 51.}

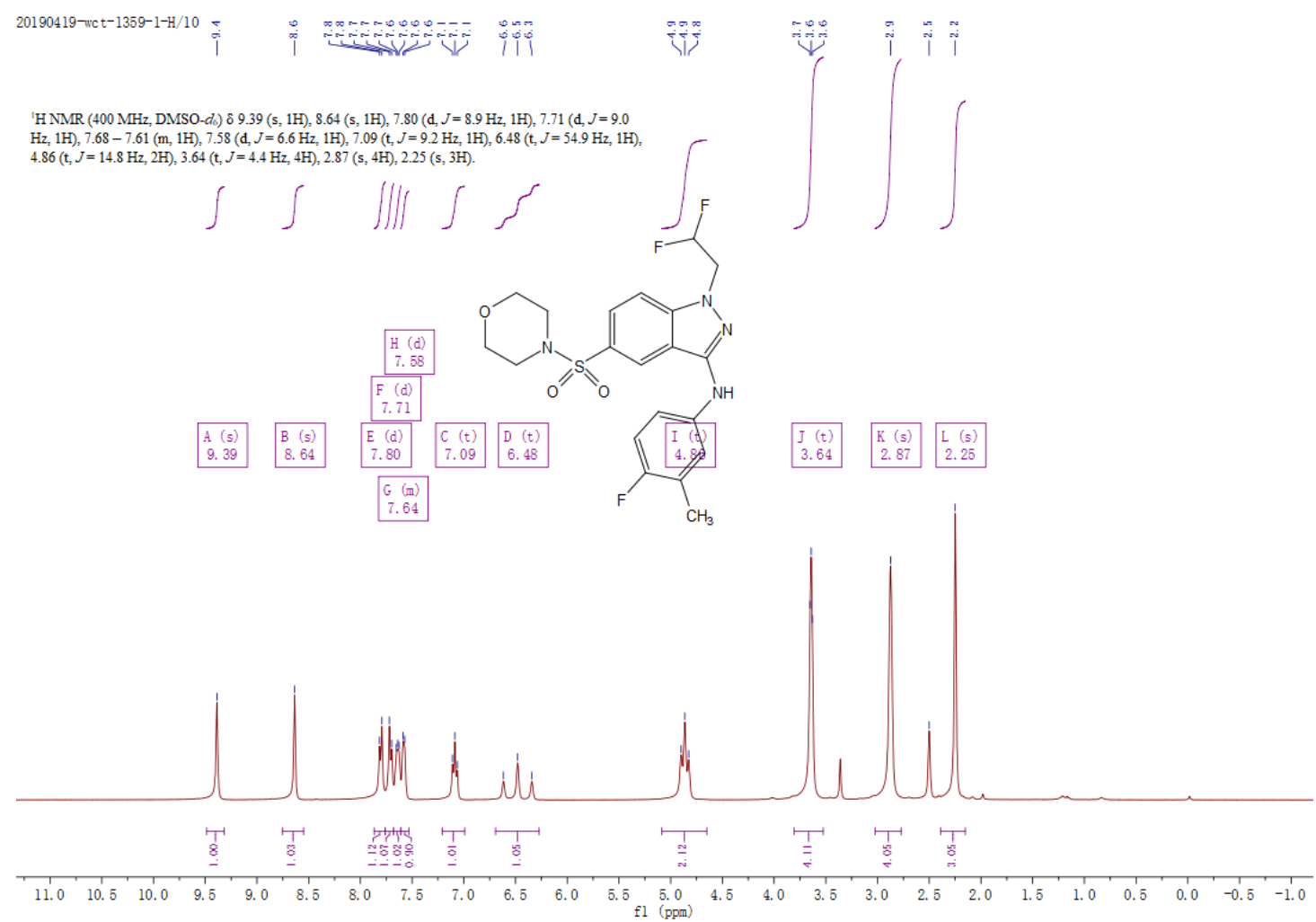

20200105-wct-1359-1-H/10

C13CPD DUSO \{D: \NUR_DAIA]\} LG 24

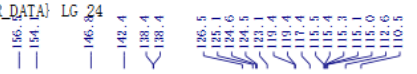

${ }^{12} \mathrm{C} \mathrm{NMR}\left(101 \mathrm{MHz}\right.$, DMSO- $\left.d_{l}\right) \delta 155.31$ (d, $\left.J=235.4 \mathrm{~Hz}\right), 146.82,142.37,138.44(\mathrm{~d}, J=2.2 \mathrm{~Hz})$,

$126.47,125.07,124.55(\mathrm{~d}, J=18.0 \mathrm{~Hz}), 123.11,119.37$ (d, $J=4.1 \mathrm{~Hz}), 115.33(\mathrm{~d}, J=14.7 \mathrm{~Hz}), 115.30$

$(\mathrm{d}, J=35.9 \mathrm{~Hz}), 114.98(\mathrm{t}, J=241.8 \mathrm{~Hz}), 110.48,65.75,50.27(\mathrm{t}, J=26.0 \mathrm{~Hz}), 46.44,15.09(\mathrm{~d}, J=2.9$
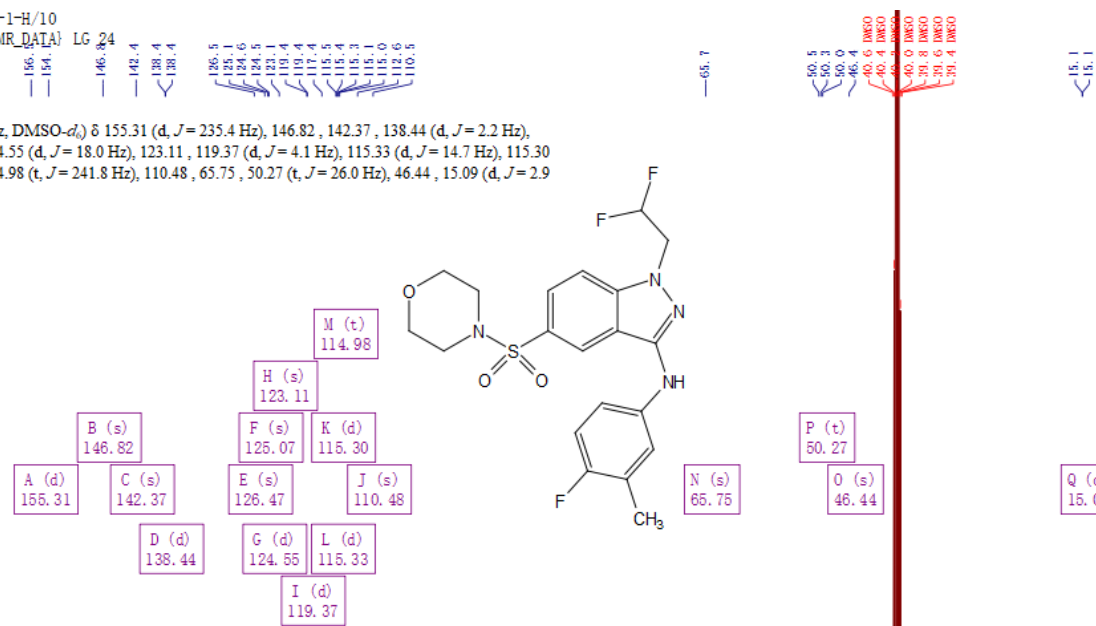

\begin{tabular}{|l|l|}
\hline$P(t)$ \\
50.27
\end{tabular}

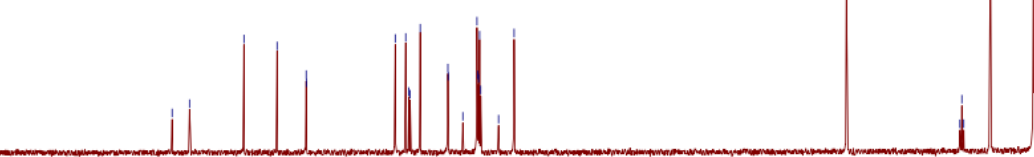

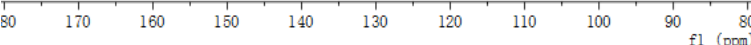




\section{Compound 52.}

\section{Tet $-1352-2-\mathrm{H} / 10$
PROTON DISO [D:}

10

H NMR (400 MHz, DMSO- $\left.d_{\text {) }}\right) 89.36$ (s, 1H), $8.63(\mathrm{~s}, 1 \mathrm{H}), 7.74(\mathrm{~d}, J=8.9 \mathrm{~Hz}, 1 \mathrm{H}), 7.66$ (dd, $J=14.4$,

(dt, $J=27.7,4.8 \mathrm{~Hz}, 2 \mathrm{H}), 3.64(\mathrm{~d}, J=4.6 \mathrm{~Hz}, 4 \mathrm{H}), 2.89(\mathrm{~d}, J=4.6 \mathrm{~Hz}, 4 \mathrm{H}), 2.26(\mathrm{~s}, 3 \mathrm{H})$

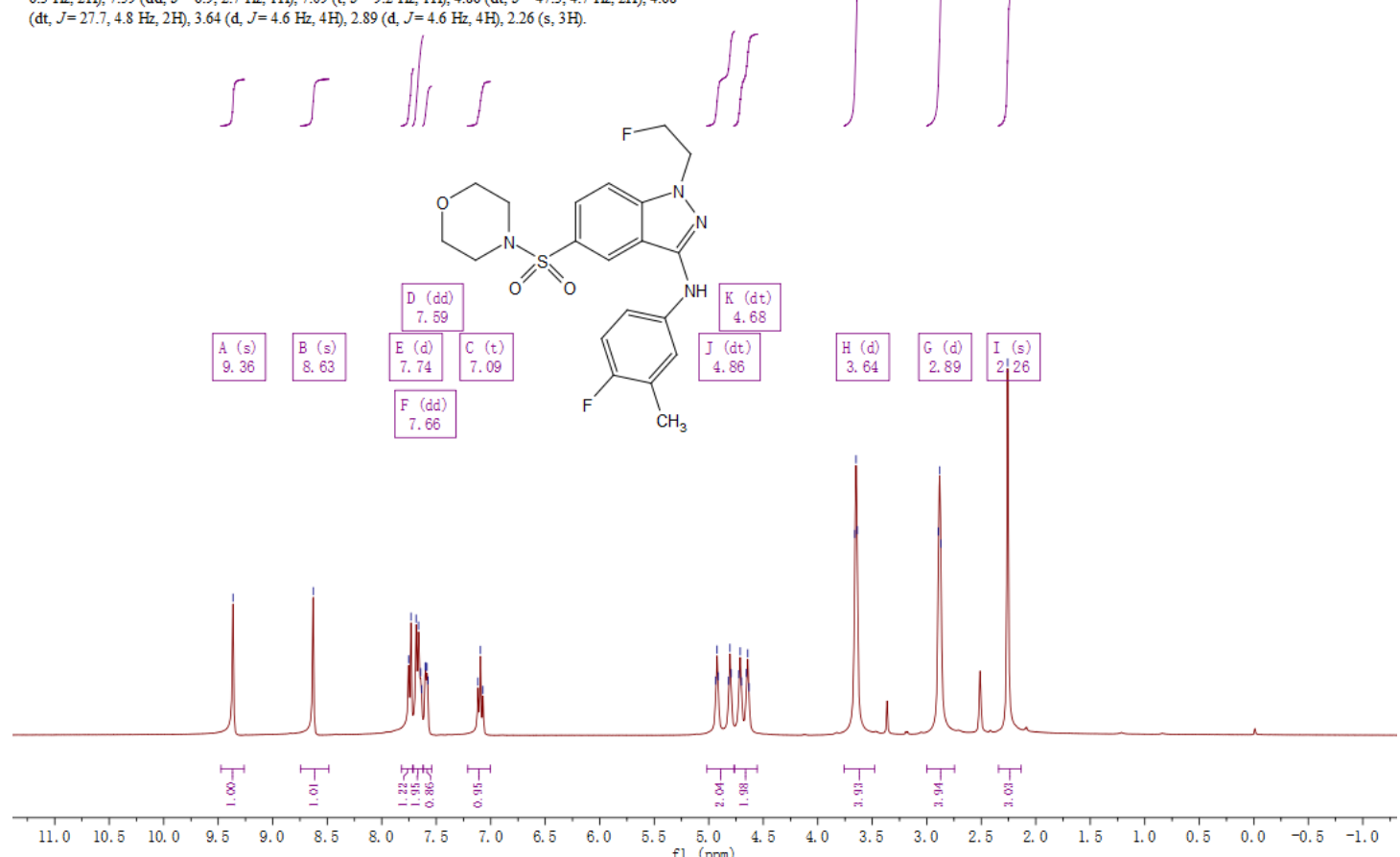

$20190412-$ wct $-1352-2-C / 10$
C13CPD DUSO \{D: $\backslash$ NIR_DATA\} LG ?

$\delta_{1} 155.23(\mathrm{~d}, J=235.1 \mathrm{~Hz}), 146.52,142.00,138.61(\mathrm{~d}, J=2.2 \mathrm{~Hz})$,

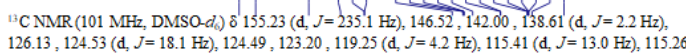
$126.13,124.53(\mathrm{~d}, J=18.1 \mathrm{~Hz}), 124.49,123.20,119.25(\mathrm{~d}, J=4.2 \mathrm{~Hz}), 115.41(\mathrm{~d}, J=13.0 \mathrm{~Hz}), 115.26$
$(\mathrm{~d}, J=2.5 \mathrm{~Hz}), 114.75,110.24,82.65(\mathrm{~d}, J=167.7 \mathrm{~Hz}), 65.75,49.04(\mathrm{~d}, J=19.9 \mathrm{~Hz}), 46.44,15.10$ (d. $(\mathrm{d}, J=2.5 \mathrm{~Hz}$
$J=2.9 \mathrm{~Hz})$
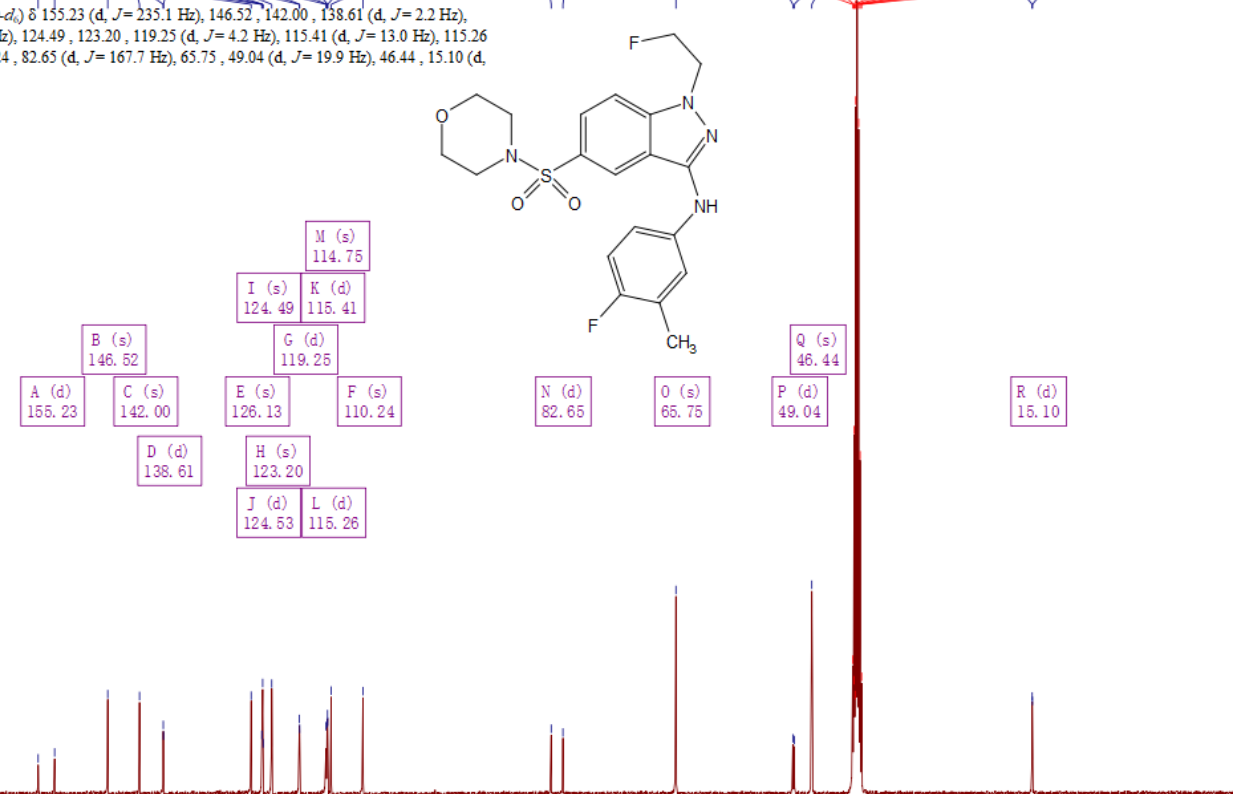

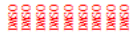

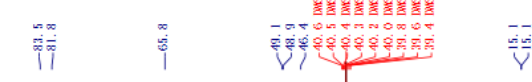

$\overline{x-\bar{x}}$

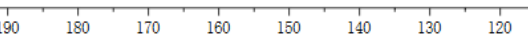

fl $\stackrel{90}{9}(\mathrm{ppm})$ 


\section{Compound 53.}

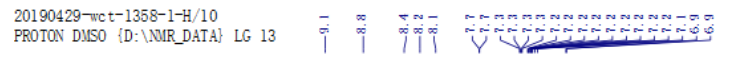

'H NMR (400 MHz, DMSO- $\left.d_{s}\right) \delta 9.11(\mathrm{~s}, 1 \mathrm{H}), 8.76(\mathrm{~s}, 1 \mathrm{H}), 8.23(\mathrm{t}, J=58.7 \mathrm{~Hz}, 1 \mathrm{H}), 7.68(\mathrm{~d}, J=1.4$ $\mathrm{Hz}, 1 \mathrm{H}), 7.29$ (dd, $J=7.0,2.5 \mathrm{~Hz}, 1 \mathrm{H}), 7.25-7.12(\mathrm{~m}, 2 \mathrm{H}), 6.87(\mathrm{~d}, J=1.4 \mathrm{~Hz}, 1 \mathrm{H}), 3.76-3.53(\mathrm{~m}$, $4 \mathrm{H}), 2.92(\mathrm{t}, J=4.7 \mathrm{~Hz}, 4 \mathrm{H}), 2.23(\mathrm{~d}, J=1.9 \mathrm{~Hz}, 3 \mathrm{H})$.

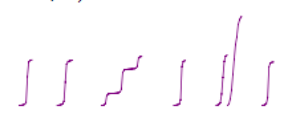

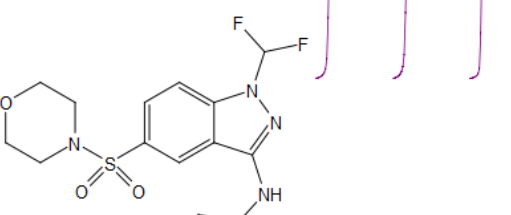

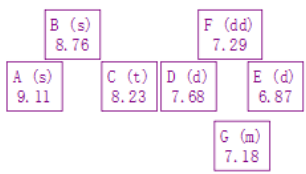<smiles>Cc1ccc(F)c(C)c1</smiles>

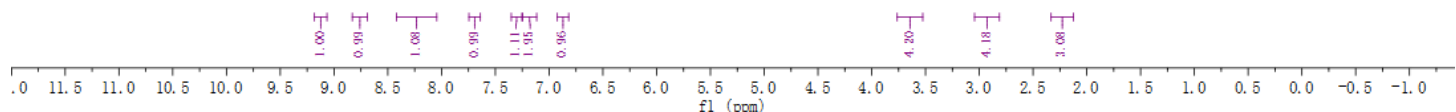
$20190419-w c t-1358-1-C / 10$
C13CPD DUSO $\{$ :D: $\backslash$ NIR__DATAL, LG 46

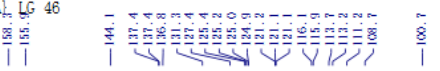

${ }^{13} \mathrm{C} \mathrm{NMR}\left(101 \mathrm{MHz}, \mathrm{DMSO}-d_{s}\right) \delta 157.09$ (d, $\left.J=238.8 \mathrm{~Hz}\right), 144.07,137.37$ (d, $\left.J=2.8 \mathrm{~Hz}\right), 136.81$, $131.30,127.44,125.31$ (d, $J=18.2 \mathrm{~Hz}), 124.93(\mathrm{~d}, J=4.8 \mathrm{~Hz}), 121.20,121.10$ (d, $J=7.8 \mathrm{~Hz}), 116.00$ (d, $J=23.1 \mathrm{~Hz}), 113.17,111.18(\mathrm{t}, J=252.6 \mathrm{~Hz}), 100.66,65.92,46.36,14.83$ (d, $J=2.9 \mathrm{~Hz})$.
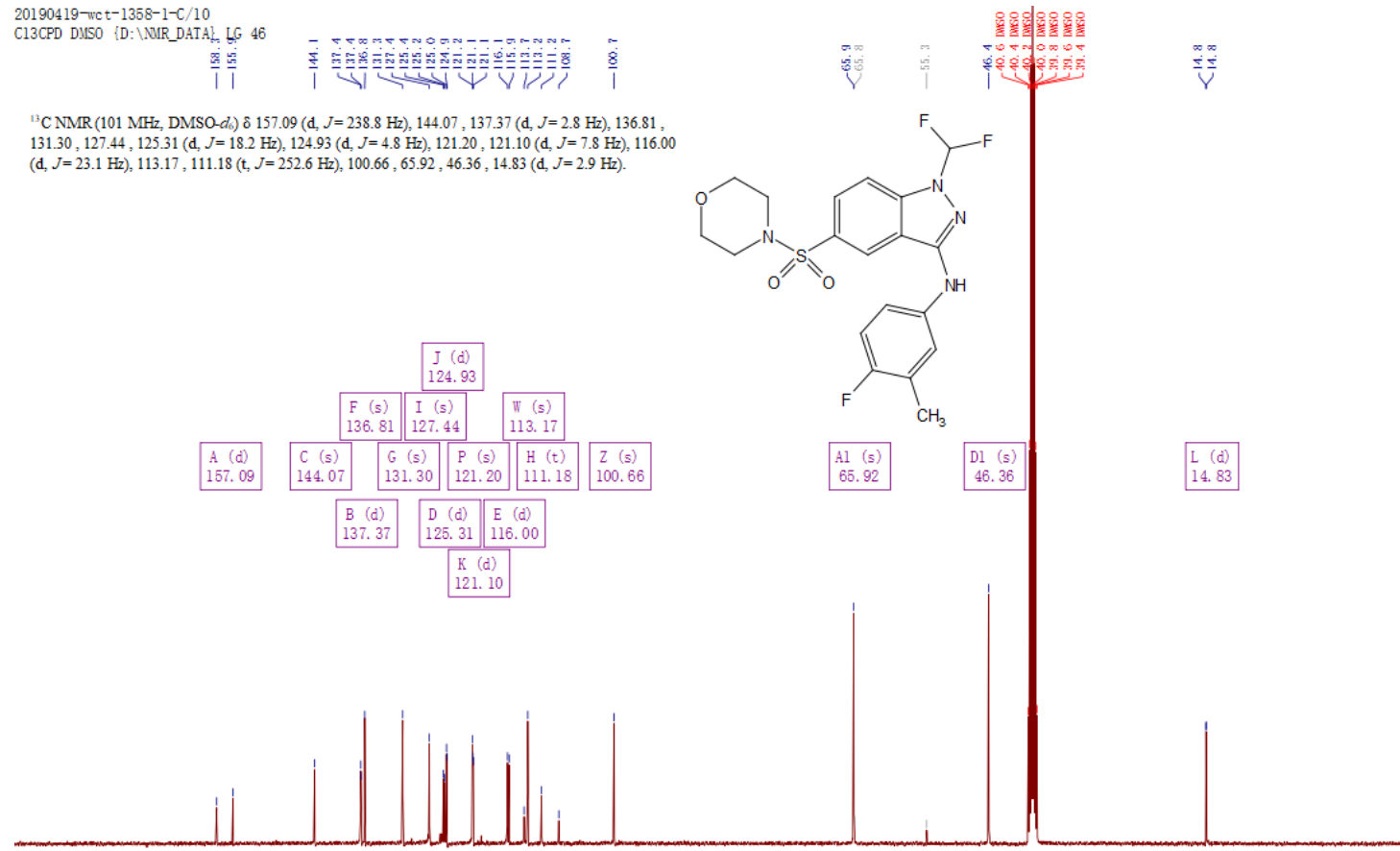

$\begin{array}{llllllllll}180 & 170 & 160 & 150 & 140 & 130 & 120 & 110 & 100 & 90 \\ \mathrm{fl}(\mathrm{ppm}) & 80\end{array}$ 


\section{Compound 54.}

20181203-wct-1263-4-H. 10, fid
PROTON DUSO \{D: \NIR_DATA\} LG

8

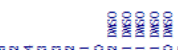

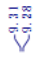

HNMR (400 MHz, DMSO-d $\left.d_{0}\right) 89.28(\mathrm{~s}, 1 \mathrm{H}), 8.67(\mathrm{~d}, J=1.6 \mathrm{~Hz}, 1 \mathrm{H}), 7.82$ (dd, $J=8.8,1.7 \mathrm{~Hz}, 1 \mathrm{H}$ ), 7.69 (d, $J=8.8 \mathrm{~Hz}, 1 \mathrm{H}), 7.64$ (ddd, $J=8.9,4.5,3.0 \mathrm{~Hz}, 1 \mathrm{H}), 7.54$ (dd, $J=6.9,2.8 \mathrm{~Hz}, 1 \mathrm{H}), 7.10$ (t, $J=$

$9.2 \mathrm{~Hz}, 1 \mathrm{H}), 4.46(\mathrm{~d}, J=3.0 \mathrm{~Hz}, 2 \mathrm{H}), 3.72-9.63(\mathrm{~m}, 2 \mathrm{H}), 3.60(\mathrm{dd}, J=7.6,1.8 \mathrm{~Hz}, 1 \mathrm{H}), 3.23(\mathrm{~d}, J=$

$10.0 \mathrm{~Hz}, 1 \mathrm{H}), 3.12(\mathrm{dd}, J=10.0,1.6 \mathrm{~Hz}, 1 \mathrm{H}), 2.25(\mathrm{~d}, J=1.97 / \mathrm{fz}, 3 \mathrm{H}), 1.62-1.49(\mathrm{~m}, 1 \mathrm{H}), 1.19-1.07$

$(\mathrm{m}, 4 \mathrm{H}), 0.96(\mathrm{dd}, J=10.1,2.3 \mathrm{~Hz}, 1 \mathrm{H})$.
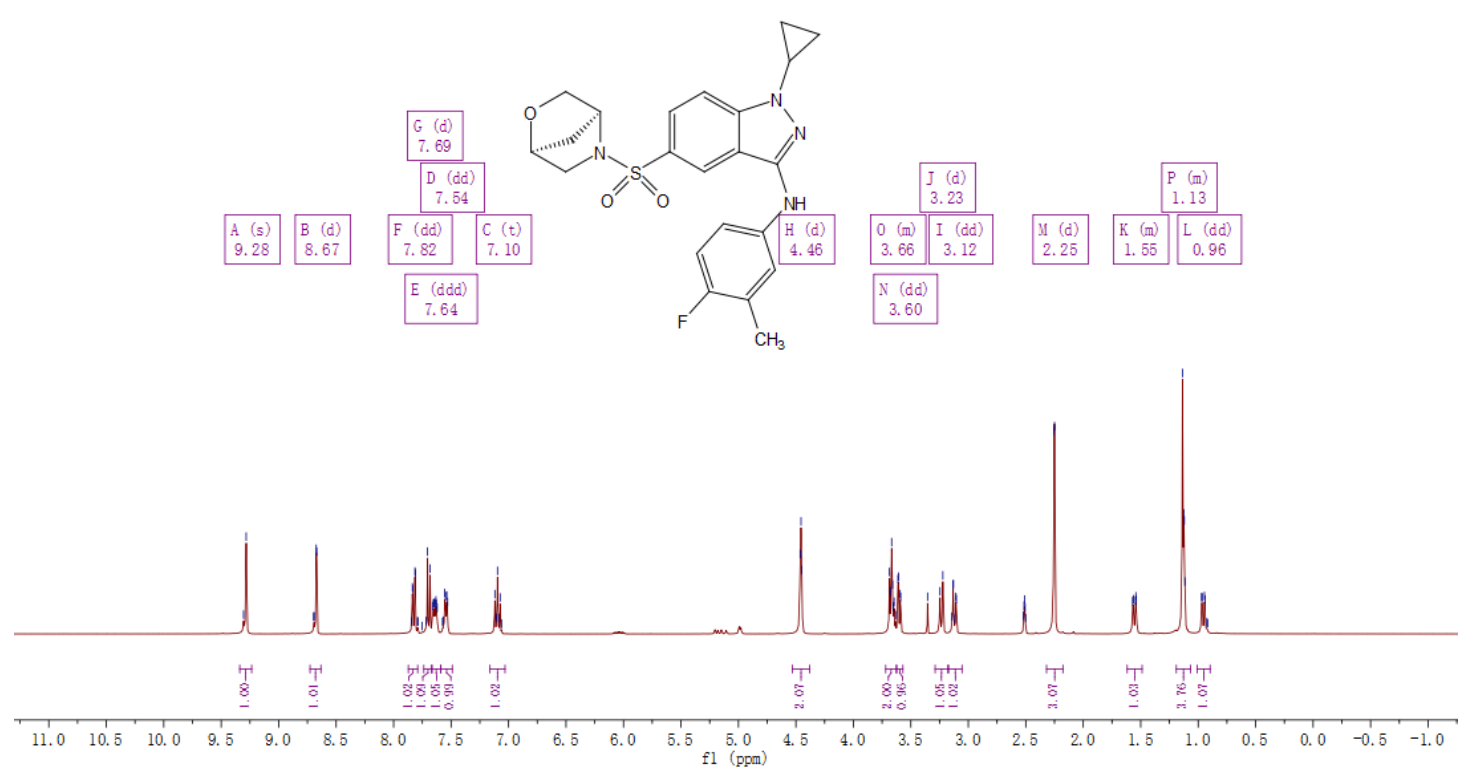

20191223-wct-1263-4-C/10

C13CPD DUSO \{D: $\backslash$ NIP D DTA

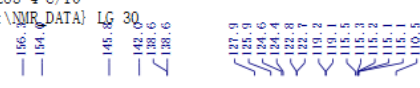

${ }^{13} \mathrm{CNMR}\left(101 \mathrm{MHz}, \mathrm{DMSO}-d_{\mathrm{l}}\right) \delta 155.17$ (d, $\left.J=235.1 \mathrm{~Hz}\right), 145.75,142.05,138.63$ (d, $\left.J=2.4 \mathrm{~Hz}\right)$,

$127.89,125.91,124.49(\mathrm{~d}, J=18.0 \mathrm{~Hz}), 122.70,119.16(\mathrm{~d}, J=4.1 \mathrm{~Hz}), 115.39(\mathrm{~d}, J=22.9 \mathrm{~Hz}), 115.18$
$($ d, $J=7.1 \mathrm{~Hz}), 115.13,110.53,76.09,73.61,60.29,56.24,35.34,29.60 .15 .12(\mathrm{~d}, J=2.9 \mathrm{~Hz}), 6.82$

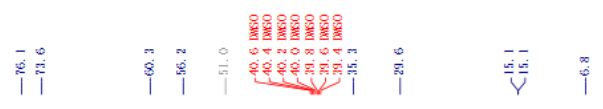
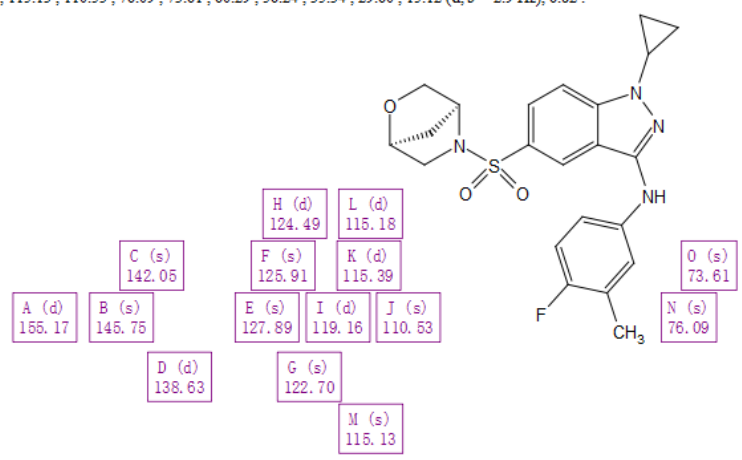

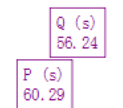

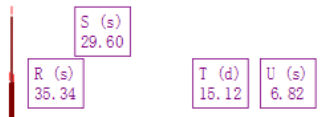

$170 \quad 160$

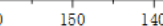

130

$120 \quad 11$

100

$90 \quad 80$ 


\section{Compound 55.}

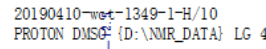

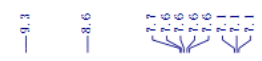

'H NMR (400 MHz, DMSO-d $\left.d_{6}\right) \delta 12.54(\mathrm{~s}, 1 \mathrm{H}), 925(\mathrm{~s}, 1 \mathrm{H}), 8.61(\mathrm{~s}, 1 \mathrm{H}), 7.78-7.49(\mathrm{~m}, 4 \mathrm{H}), 7.07(\mathrm{t}$, $J=9.3 \mathrm{~Hz}, 1 \mathrm{H}), 3.65(\mathrm{~s}, 5 \mathrm{H}), 2.88(\mathrm{~s}, 4 \mathrm{H}), 2.25(\mathrm{~s}, 3 \mathrm{H})$.
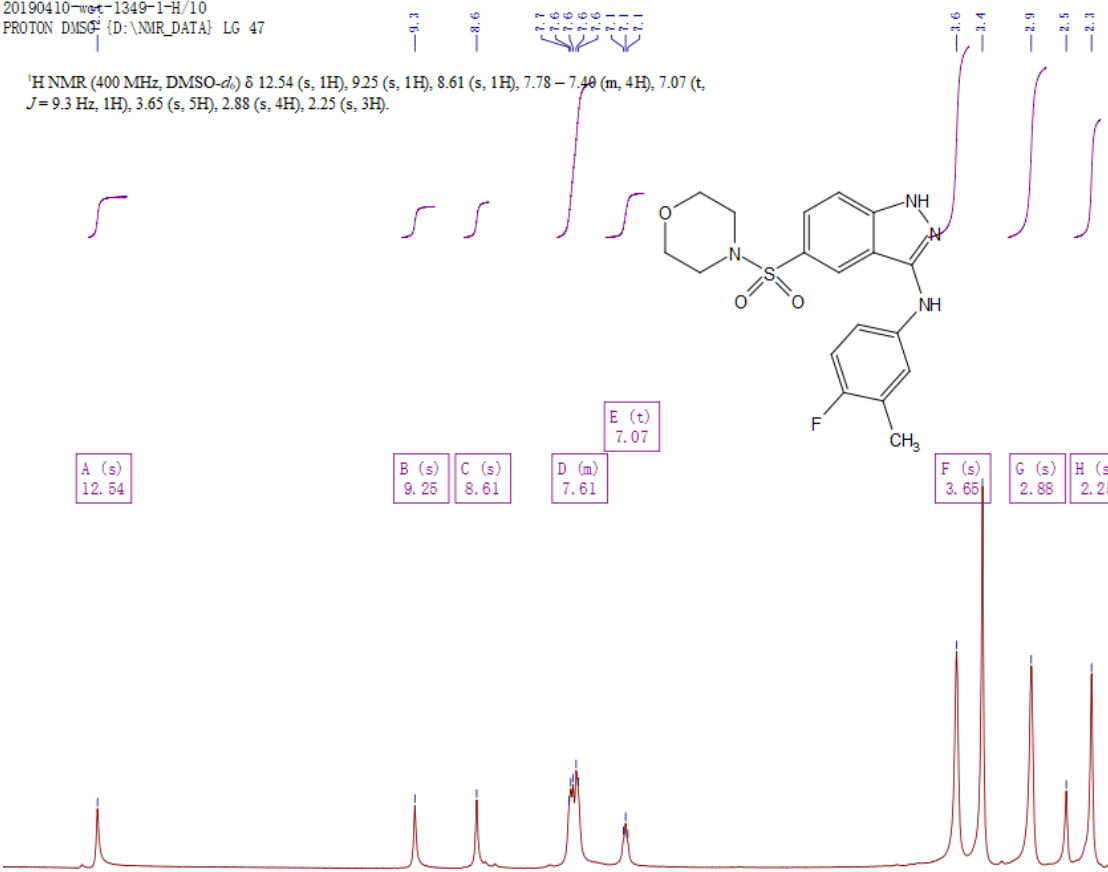

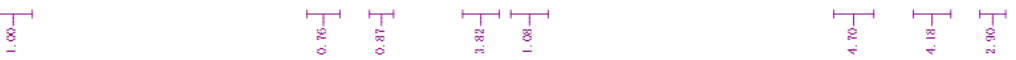

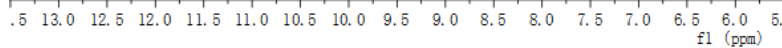

$20190411-w c t-1349-1-\mathrm{C} / 10$

| ।

${ }^{13} \mathrm{C} \mathrm{NMR}\left(101 \mathrm{MHz}, \mathrm{DMSO}-d_{l}\right) \delta 155.04(\mathrm{~d}, J=234.5 \mathrm{~Hz}), 146.88,141.92,138.95,125.90,124.40$

$(\mathrm{d}, J=17.8 \mathrm{~Hz}), 124.19,123.09,119.00$ (d, $J=3.9 \mathrm{~Hz}), 115.36(\mathrm{~d}, J=22.2 \mathrm{~Hz}), 115.18,114.14,110.73$

$, 65.76,46.45,15.09(\mathrm{~d}, J=3.1 \mathrm{~Hz})$
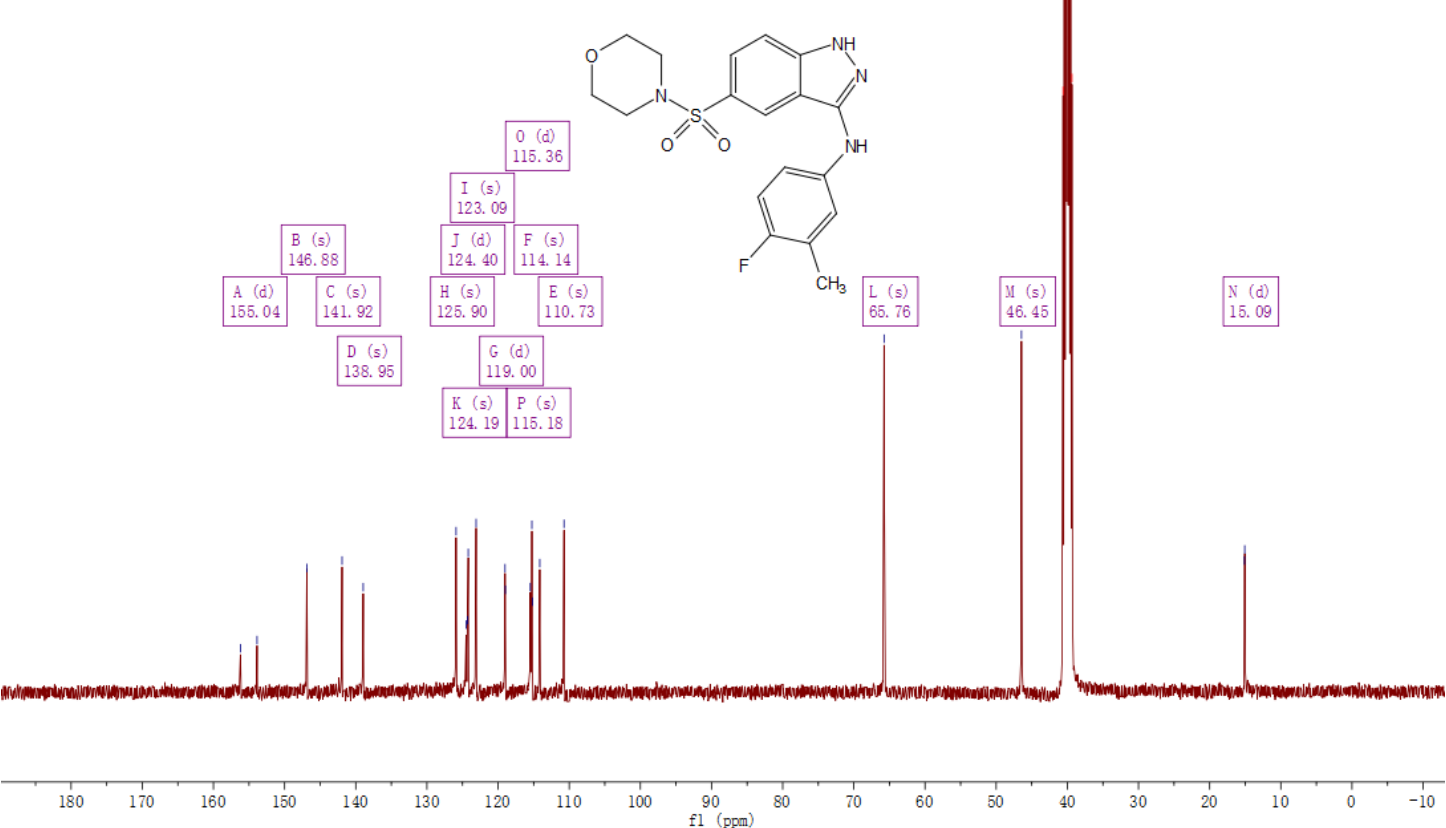


\section{Compound 56.}

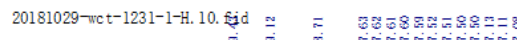

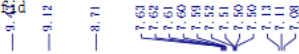

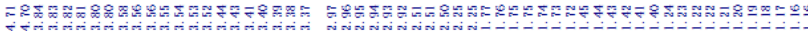

'H NMR (400 MHz, DMSO-d $\left.\mathrm{d}_{\mathrm{s}}\right) 89.43$ (s, $\left.1 \mathrm{H}\right), 9.12(\mathrm{~s}, 1 \mathrm{H}), 8.71(\mathrm{~s}, 1 \mathrm{H}), 7.61$ (dt, $\left.J=8.3,3.6 \mathrm{~Hz}, 1 \mathrm{H}\right)$, $7.51(\mathrm{dd}, J=6.8,2.8 \mathrm{~Hz}, 1 \mathrm{H}), 7.11(\mathrm{t}, J=9.2 \mathrm{~Hz}, 1 \mathrm{H}), 4.70(\mathrm{~d}, J=3.9 \mathrm{~Hz}, 1 \mathrm{H}), 3.82$ (dq, $J=7.1,3.5 \mathrm{~Hz}$ $\mathrm{lH}), 3.55(\mathrm{tt}, J=8.1,4.6 \mathrm{~Hz}, 1 \mathrm{H}), 3.48-3.37(\mathrm{~m}, 2 \mathrm{H}), 2.94(\mathrm{ddd}, J=12.2,8.9,3.4 \mathrm{~Hz}$
$(\mathrm{m}, 3 \mathrm{H}), 1.83-1.68(\mathrm{~m}, 2 \mathrm{H}), 1.42(\mathrm{dtd}, J=12.6,8.3,3 \mathrm{~Hz}, 2 \mathrm{H}), 1.28-1.13(\mathrm{~m}, 4 \mathrm{H})$

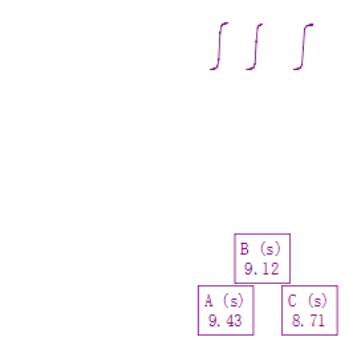<smiles>C=CCCCCCCCCCC</smiles>
$f(1) \int j$<smiles>CCNCC1=NC=C2C(C1)NN2C1CC1</smiles><smiles>C1CCC2CCCCC2C1</smiles>
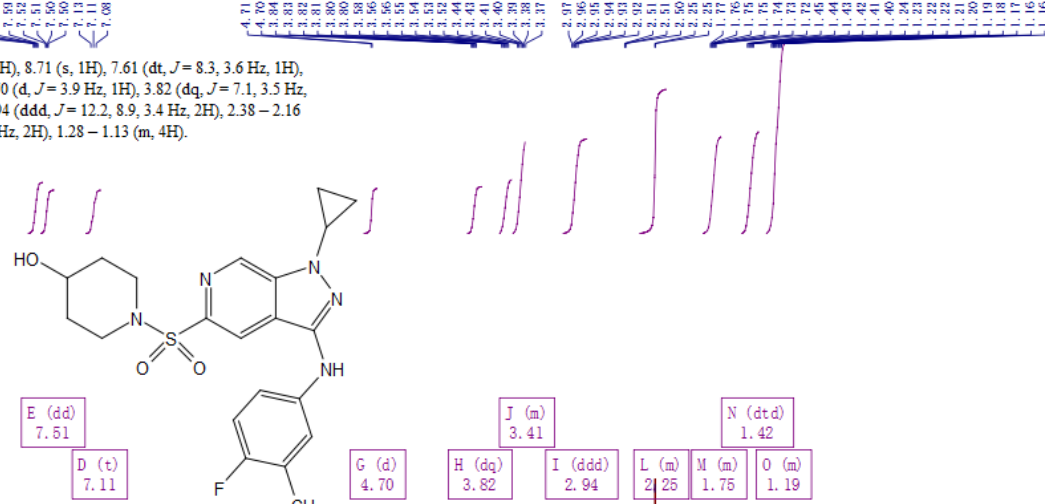

$\mathrm{F}(\mathrm{dt})$
7.61

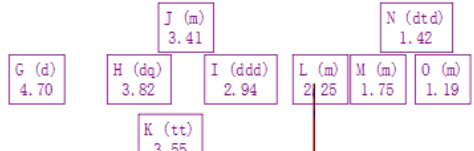<smiles>Cc1ccc(F)c(C)c1</smiles>

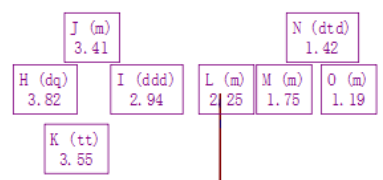

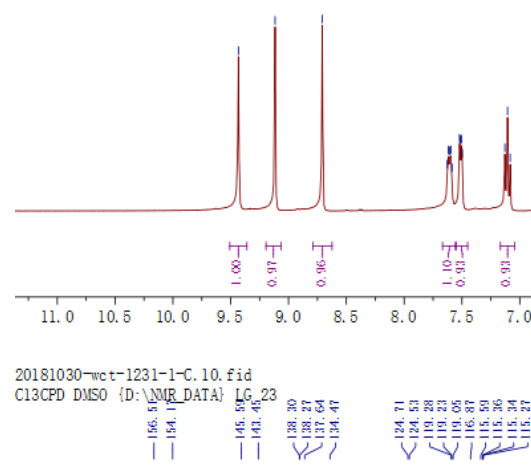

${ }^{3} \mathrm{CNMR}(101 \mathrm{MHz}$, DMSO-d $\mathrm{d}) 8155.34$ (d, $\left.J=235.5 \mathrm{~Hz}\right), 145.59,143.45,138.29$ (d, $\left.J=2.3 \mathrm{~Hz}\right)$ $137.64,134.47,124.62(\mathrm{~d}, J=18.2 \mathrm{~Hz}), 119.26(\mathrm{~d}, J=4.3 \mathrm{~Hz}), 119.05,116.87,115.59,115.36,115.30$ (d, $J=7.2 \mathrm{~Hz}), 64.79,44.18,33.77,30.19,15.11(\mathrm{~d}, J=3.0 \mathrm{~Hz}), 6.94$.

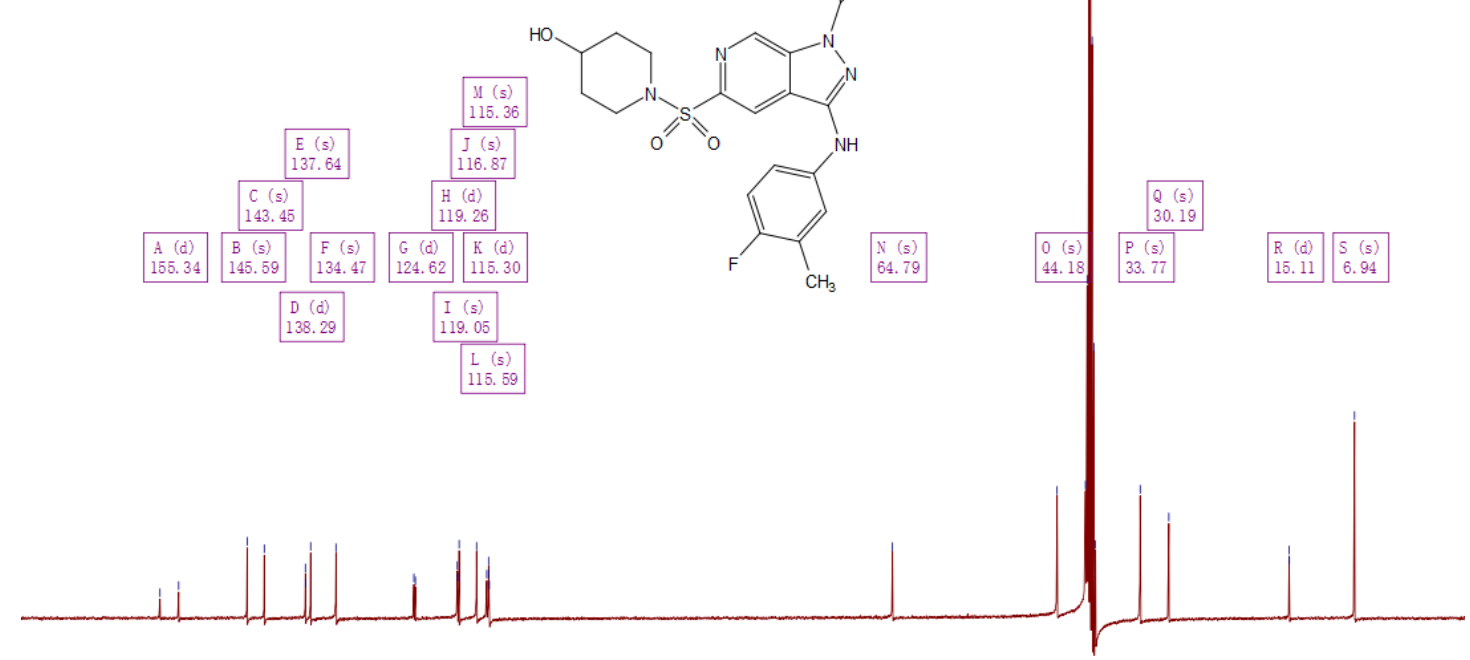

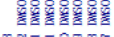

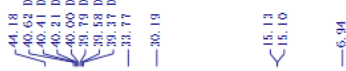

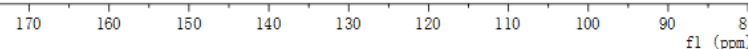




\section{Compound 57.}

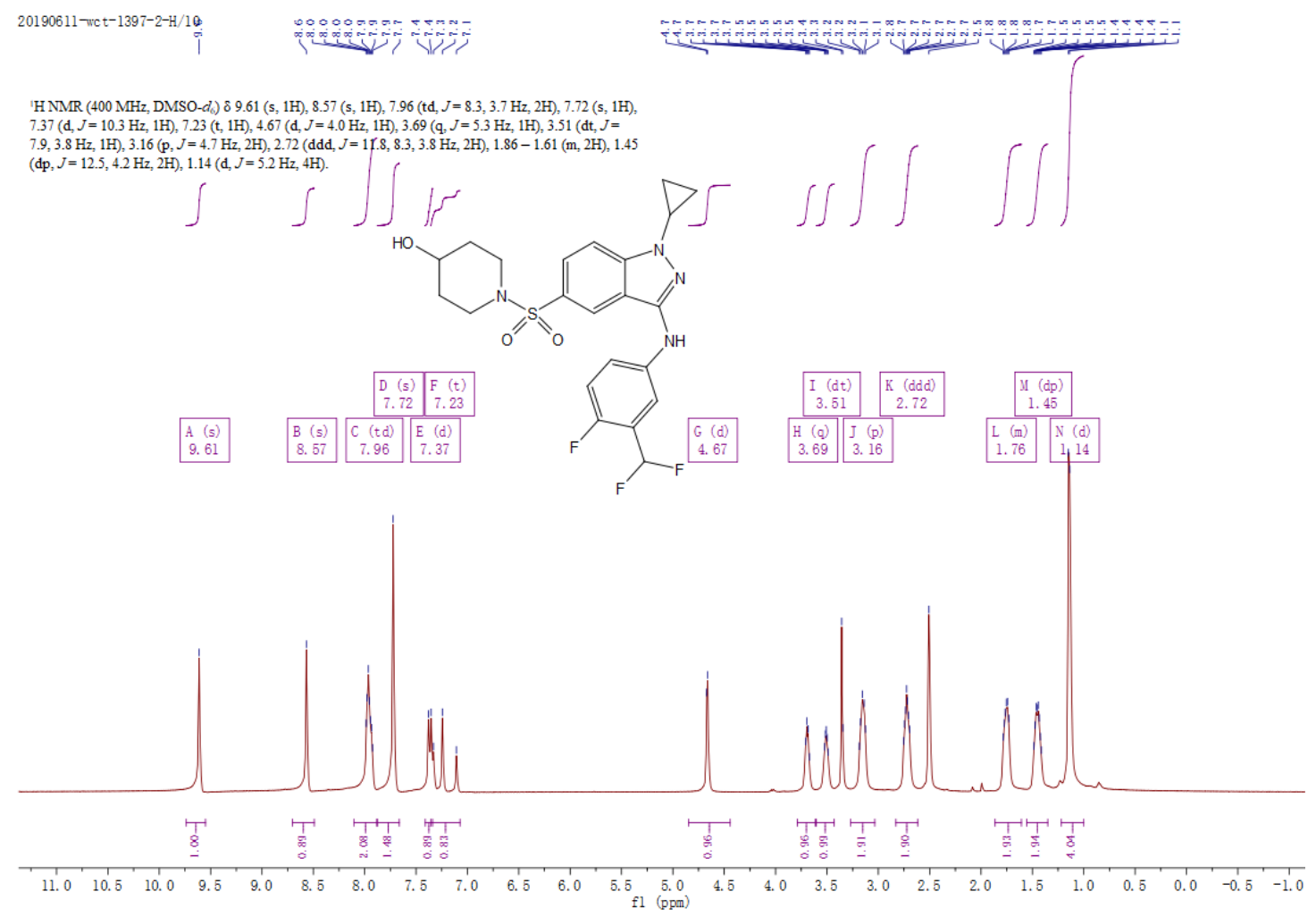

20190611-wct $-1397-2-C / 10$
C13CPD DUSO \{D: $:$ NNR_DATA\} LG, $16_{\infty}$

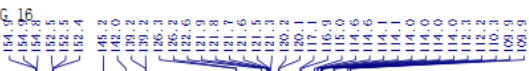

${ }^{13} \mathrm{C} \mathrm{NMR}\left(101 \mathrm{MHz}, \mathrm{DMSO}-d_{\mathrm{l}}\right) \delta 153.66$ (dt, $\left.J=242.3,5.6 \mathrm{~Hz}\right), 145.25,142.04,139.20$ (d, $J=2.2$

$\mathrm{Hz}), 126.26,126.20,122.65,121.62$ (td, $J=22.7,13.5 \mathrm{~Hz}), 120.14$ (d, $J=7.3 \mathrm{~Hz}), 117.00$ (d, $J=21$.

$\mathrm{Hz}), 115.05,114.04$ (dt $J=6.4,3.5 \mathrm{~Hz}), 112.26$ (tdd $J=236.0,3.8 \mathrm{~Hz}), 110.33,64.24,43.71,33.37$,

$29.64,6.78$.
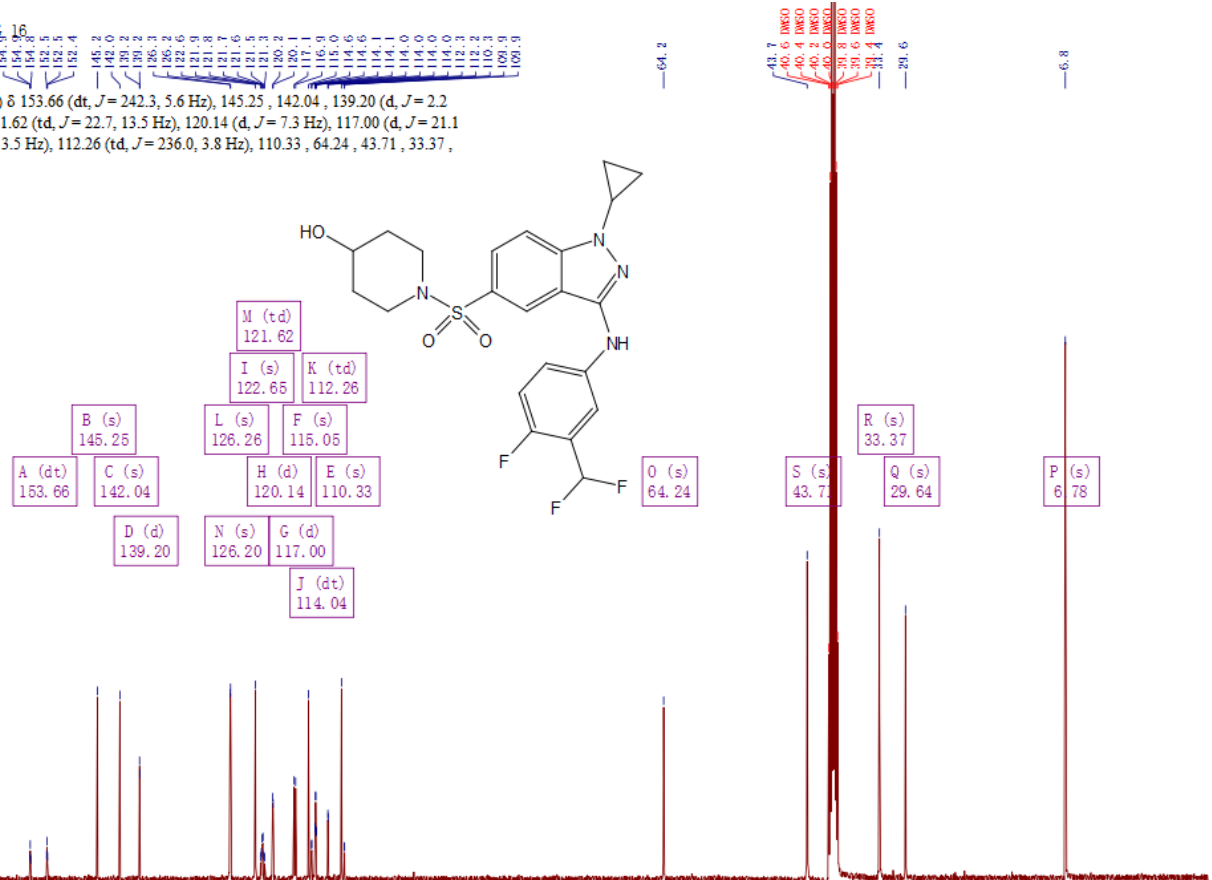

114. 04

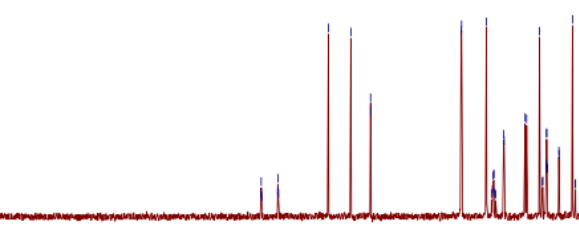

190

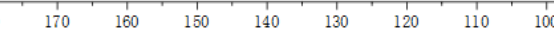

90

$80 \quad 70$

60

40

$20,10 \quad 0 \quad-10$ 


\section{Compound 58.}

\section{1 wct $-1399-1-\mathrm{H} / 10$}

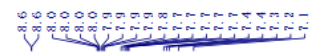

'H NMR (400 MHz, DMSO- $\left.d_{2}\right) \delta 9.63(\mathrm{~s}, 1 \mathrm{H}), 8.58(\mathrm{~d}, J=1.7 \mathrm{~Hz}, 1 \mathrm{H}), 8.04-7.88(\mathrm{~m}, 2 \mathrm{H}), 7.81-7.66$ $(\mathrm{m}, 2 \mathrm{H}), 7.40-7.09(\mathrm{~m}, 2 \mathrm{H}), 3.71(\mathrm{p}, J=5.4 \mathrm{~Hz}, 1 \mathrm{H}), 3.68-3.58(\mathrm{~m}, 4 \mathrm{H}), 2.91-2.82(\mathrm{~m}, 4 \mathrm{H}), 1.20-$ $1.08(\mathrm{~m}, 4 \mathrm{H})$.

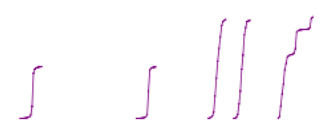

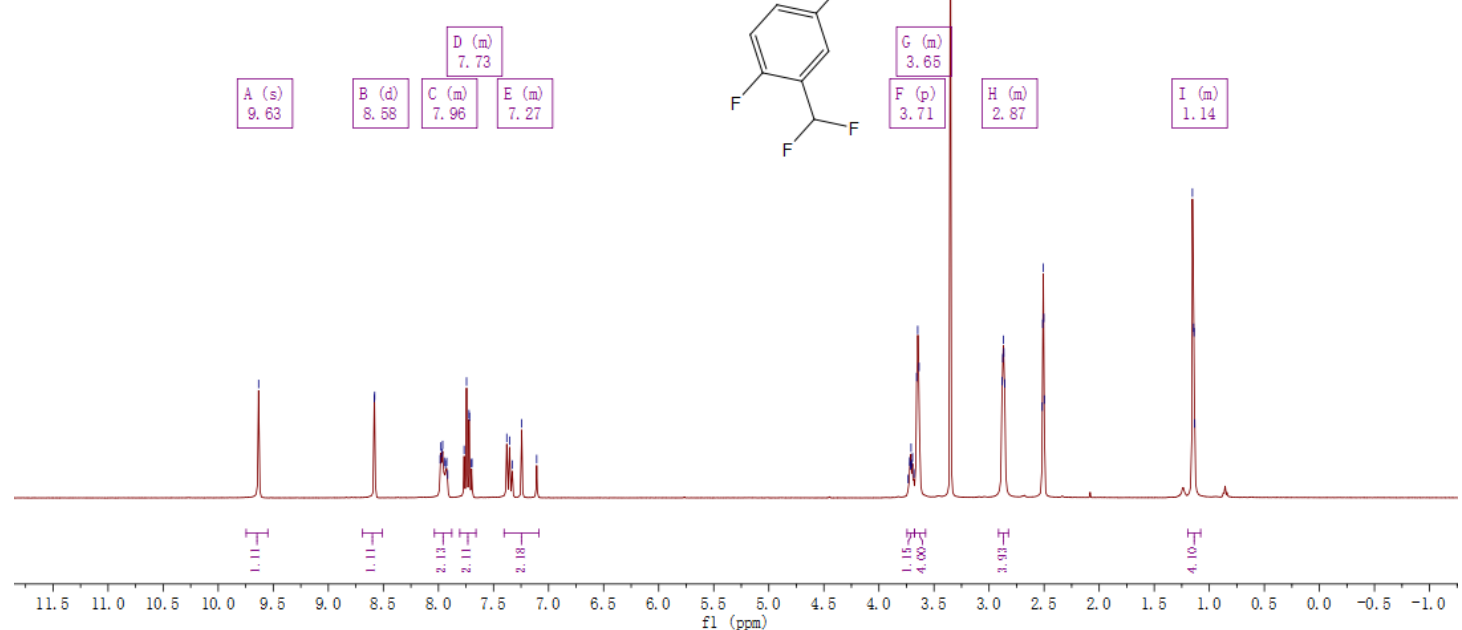<smiles>C/C=C(/CC)S(=O)(=O)N1CCOCC1</smiles>

$20190614-w c t-1399-1-C / 10$

CIXCPD DMSO \{D: WNIR_DATA\} L

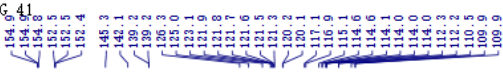

${ }^{13} \mathrm{C} \mathrm{NMR}\left(101 \mathrm{MHz}\right.$, DMSO- $d_{0}$ ) $\delta 153.67$ (dt, $\left.J=242.3,5.7 \mathrm{~Hz}\right), 145.31,142.14,139.17$ (d, $J=2.3$

$\mathrm{Hz}), 126.29,125.02,123.07,121.63(\mathrm{td}, J=22.7,13.5 \mathrm{~Hz}), 120.16(\mathrm{~d}, J=7.6 \mathrm{~Hz}), 117.01(\mathrm{~d}, J=21.3$

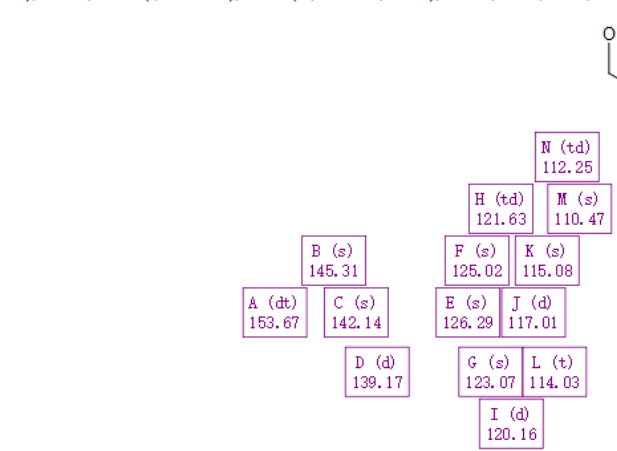<smiles>Nc1nn(C2CC2)c2ccc(S(=O)(=O)N3CCOCC3)cc12</smiles>

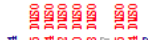

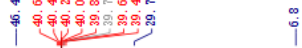

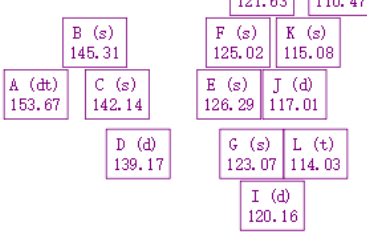
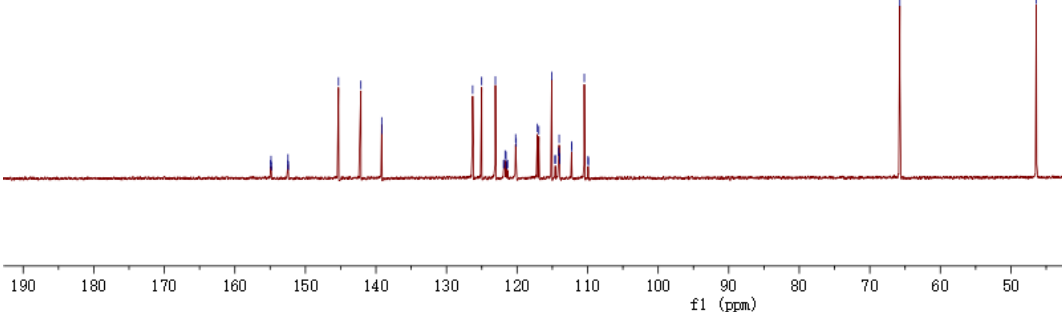

$\stackrel{90}{90}$

1
80

(1) 


\section{Compound 59.}

20190823-wct-1398-2-H/10
PROTON DUSO \{D: LNR__DATA\} LG 31

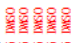

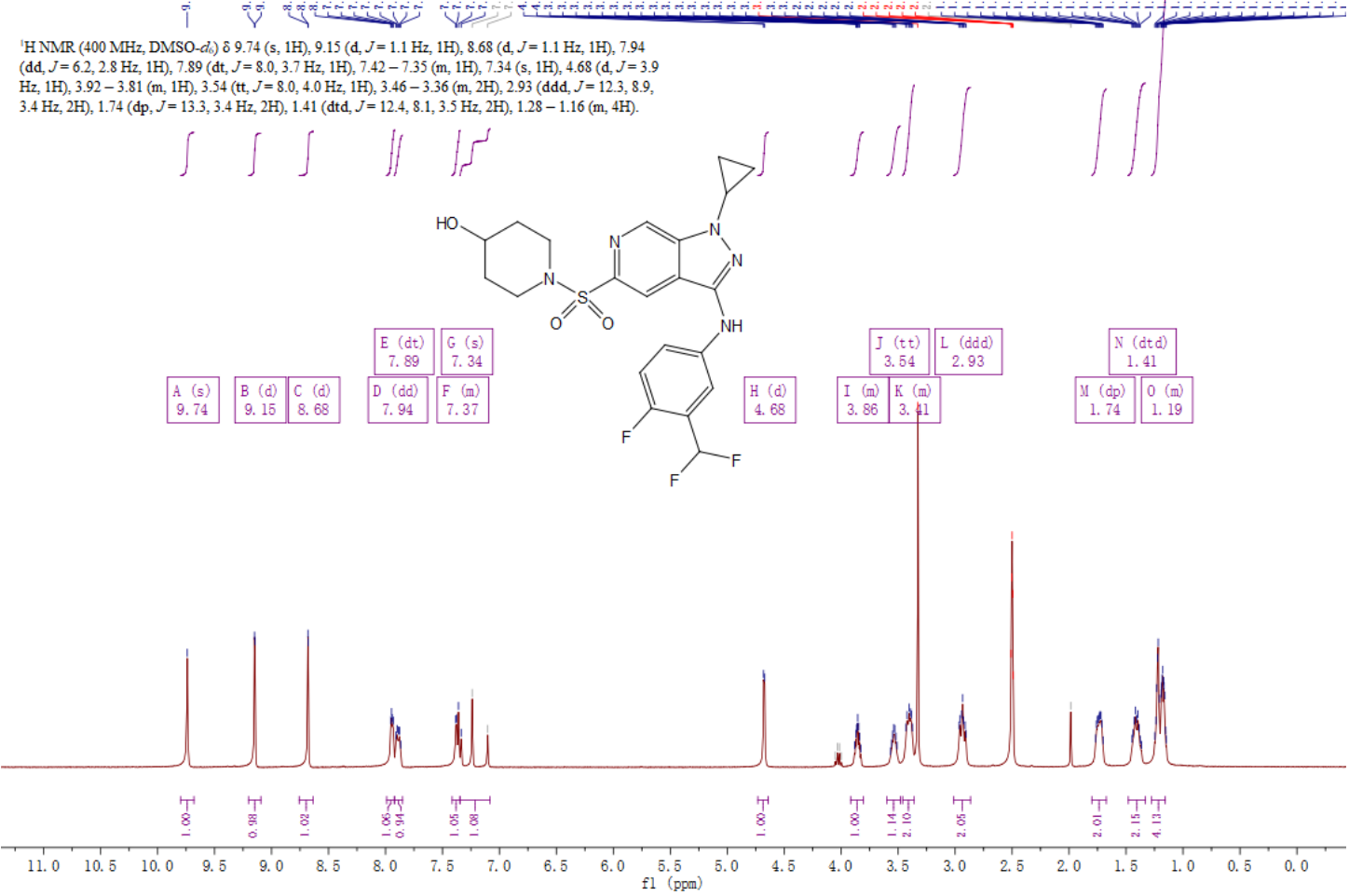

20190611-wct-1398-2-C/10
C13CPD DUSO [D: \NIR_DATA\} LG 18 o

${ }^{12} \mathrm{CNMR}\left(101 \mathrm{MHz}, \mathrm{DMSO}-d_{6}\right) \delta 153.83(\mathrm{~d}, J=243.0 \mathrm{~Hz}), 145.15,143.64,138.83(\mathrm{~d}, J=2.3 \mathrm{~Hz})$,

$137.61,134.69,122.32-121.19(\mathrm{~m}), 120.28(\mathrm{~d}, J=7.5 \mathrm{~Hz}), 118.91,117.16(\mathrm{~d}, J=21.3 \mathrm{~Hz}), 116.71$

$114.06(\mathrm{~d}, J=5.6 \mathrm{~Hz}), 114.87-109.48(\mathrm{~m}), 64.77,44.17,33.76,30.28,6.90$.

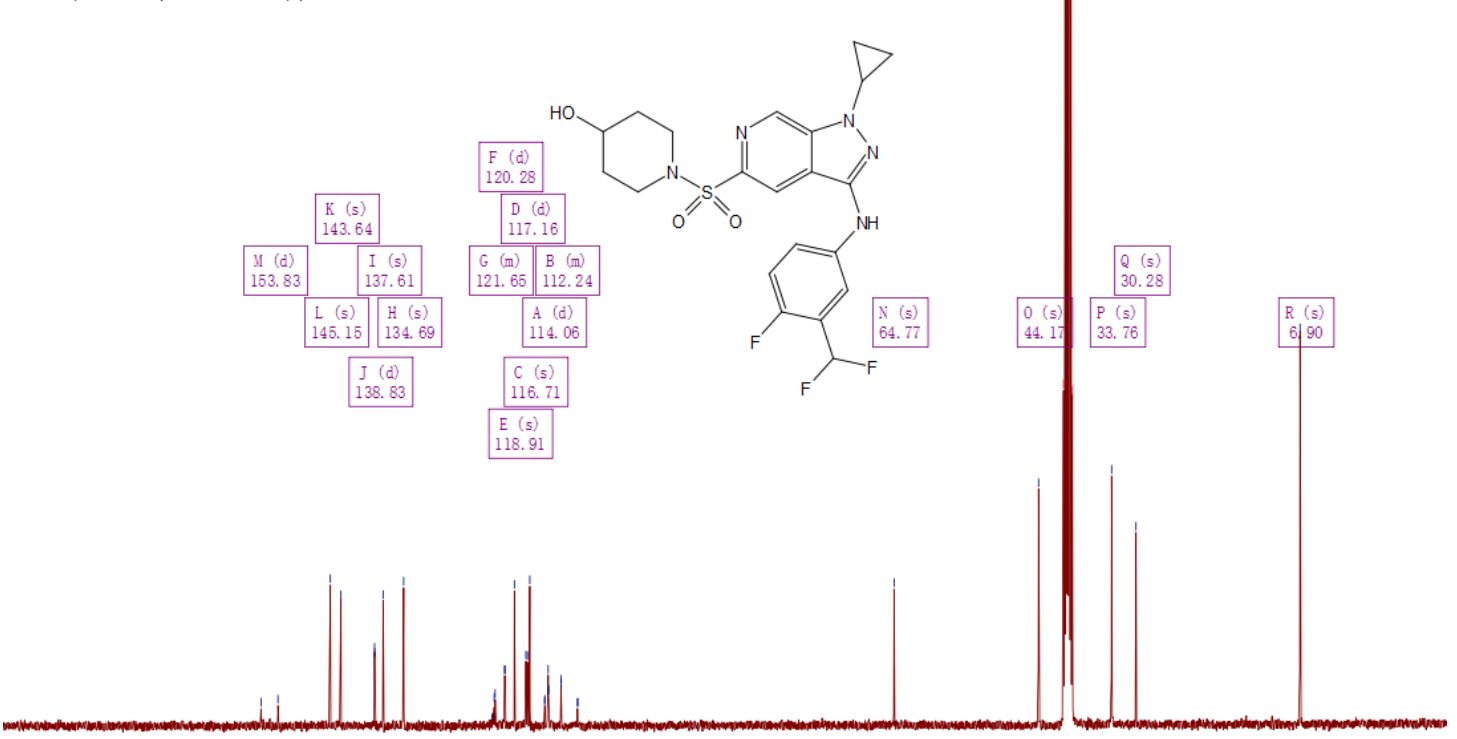

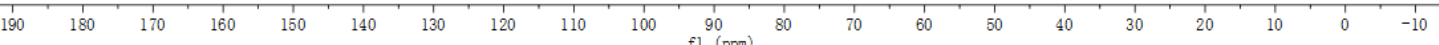




\section{Compound 60.}

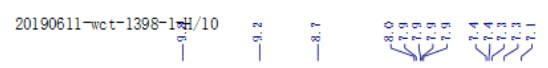

'H NMR (400 MHz, DMSO- $\left.d_{6}\right) \delta 9.78(\mathrm{~s}, 1 \mathrm{H}), 9.17(\mathrm{~s}, 1 \mathrm{H}), 8.71(\mathrm{~s}, 1 \mathrm{H}), 7.95(\mathrm{~d}, J=5.7 \mathrm{~Hz}, 1 \mathrm{H}), 7.93$ $-7.84(\mathrm{~m}, 1 \mathrm{H}), 7.38(\mathrm{~d}, J=8.2 \mathrm{~Hz}, 1 \mathrm{H}), 7.36-7.09(\mathrm{~m}, 1 \mathrm{H}), 3.86(\mathrm{q}, J=4.7,3.5 \mathrm{~Hz}, 1 \mathrm{H}), 3.63(\mathrm{t}, J=4.4$ $\mathrm{Hz}, 4 \mathrm{H}), 3.13(\mathrm{t}, J=4.5 \mathrm{~Hz}, 4 \mathrm{H}), 1.34-1.09(\mathrm{~m}, 4 \mathrm{H})$.<smiles>C=CCCCCCCCCCC</smiles>

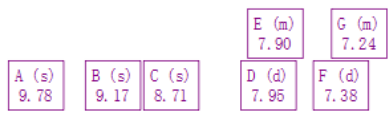<smiles>O=S(=O)(c1cc2c(Nc3ccc(F)c(C(F)F)c3)nn(C3CC3)c2cn1)N1CCOCC1</smiles>

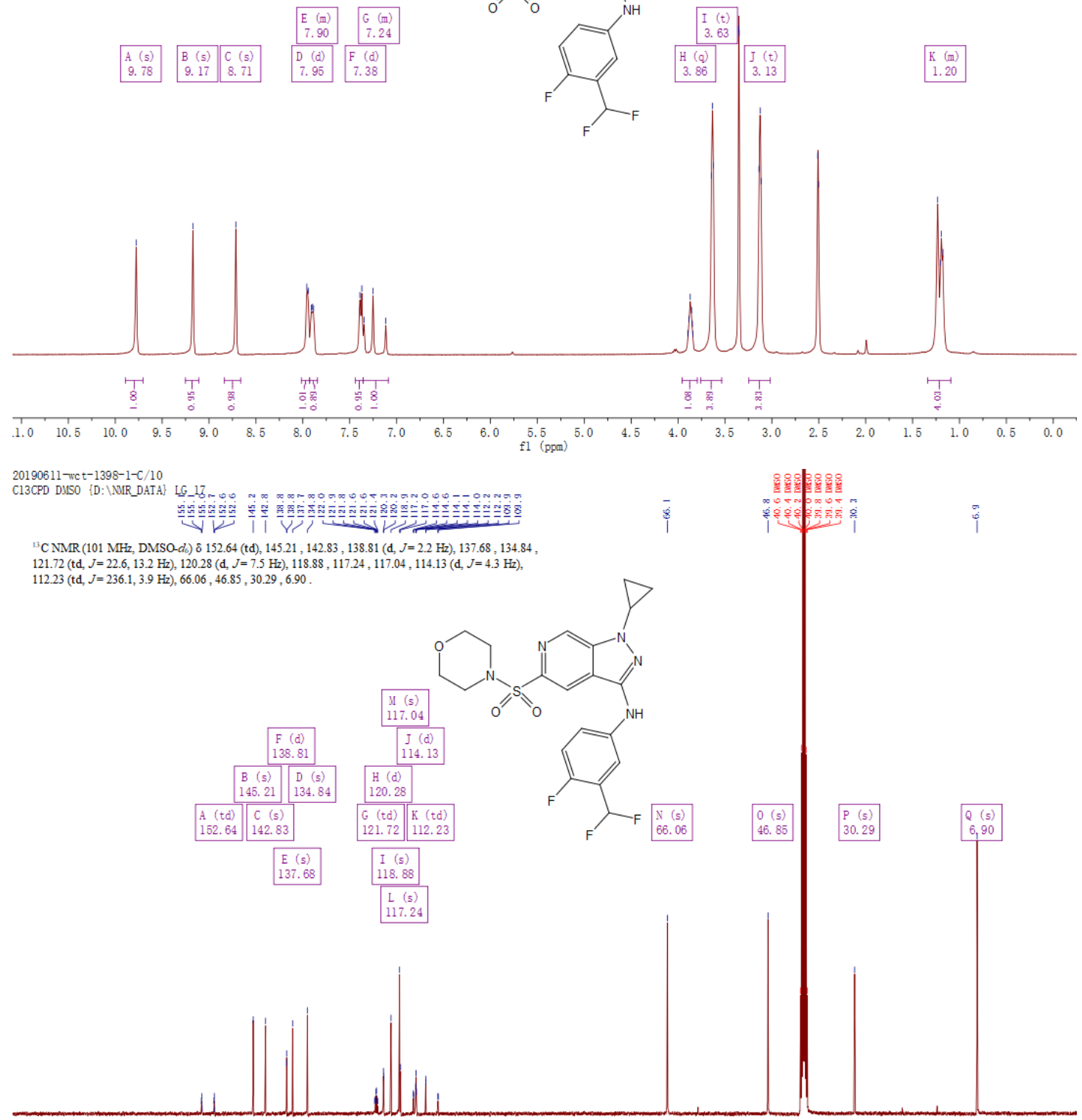

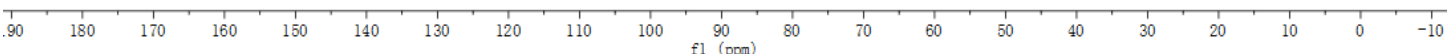




\section{Compound 61.}

20191107-wct-1450-1-H/10
PROTON DISO [D: LNIR_DATA\} LG 46

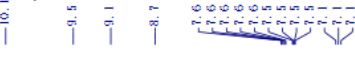

"H NMR ( $\left.400 \mathrm{MHz}, \mathrm{DMSO}-\mathrm{d}_{\mathrm{s}}\right) \delta 10.06(\mathrm{~s}, 1 \mathrm{H}), 9.46(\mathrm{~s}, 1 \mathrm{H}), 9.13(\mathrm{~s}, 1 \mathrm{H}), 8.74(\mathrm{~s}, 1 \mathrm{H}), 7.61$ (dt, $J=$

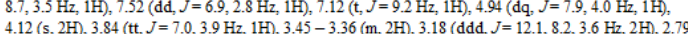

$4.12(\mathrm{~s}, 2 \mathrm{H}), 3.84(\mathrm{tt}, J=7.0,3.9 \mathrm{~Hz}, 1 \mathrm{H}), 3.45-3.36(\mathrm{~m}, 2 \mathrm{H}), 3.18(\mathrm{ddd}, J=12.1,8.2,3.6 \mathrm{~Hz}, 2 \mathrm{H}), 2.7$
$(\mathrm{~s}, 6 \mathrm{H}), 2.26(\mathrm{~d}, J=1.8 \mathrm{~Hz}, 3 \mathrm{H}), 2.01-1.85(\mathrm{~m}, 2 \mathrm{H}), 1.68(\mathrm{q}, J=9.4 \mathrm{~Hz}, 2 \mathrm{H}), 1.26-1.13(\mathrm{~m}, 4 \mathrm{H})$.

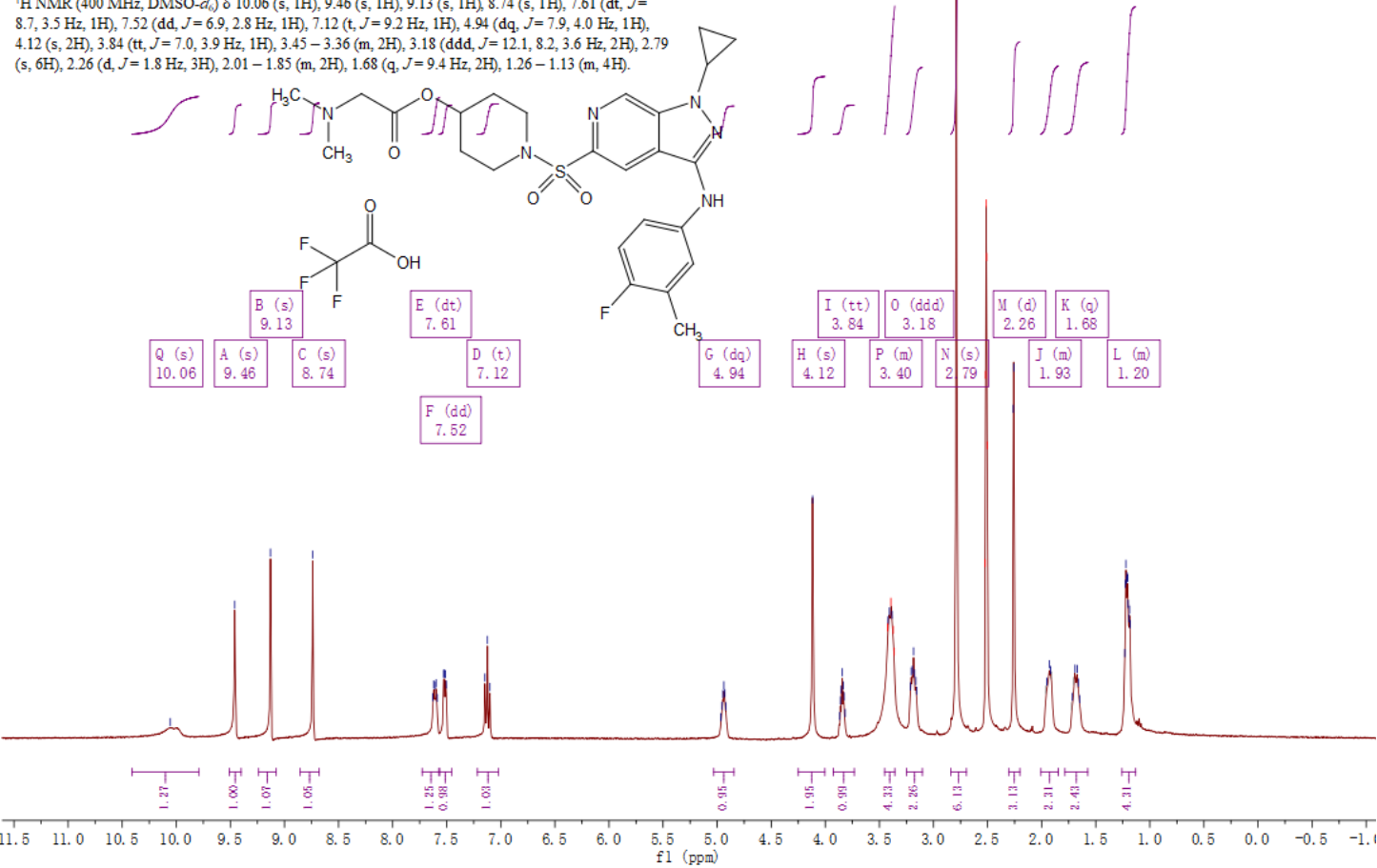




\section{Compound 62}

20191107-wct-1451-1-H/10
PROTON DUSO [D: $\backslash$ NMR_DATA\} LG 47

$$
\text { iे }
$$

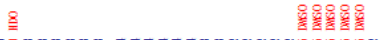

'H NMR (400 MHz, DMSO-d $d_{\text {) }} \delta 9.45$ (s, $\left.1 \mathrm{H}\right), 9.10$ (d, $\left.J=1.1 \mathrm{~Hz}, 1 \mathrm{H}\right), 8.73$ (d, $\left.J=1.1 \mathrm{~Hz}, 1 \mathrm{H}\right), 8.30$

$(\mathrm{s}, 3 \mathrm{H}), 7.60(\mathrm{dt}, J=8.7,3.6 \mathrm{~Hz}, 1 \mathrm{H}), 7.50(\mathrm{dd}, J=6.9,2.8 \mathrm{~Hz}, 1 \mathrm{H}), 7.11(\mathrm{t}, J=9.2 \mathrm{~Hz}, 1 \mathrm{H}), 4.93(\mathrm{tt}, J=$

$7.3,3.6 \mathrm{~Hz}, 1 \mathrm{H}), 3.92-3.86(\mathrm{~m}, 1 \mathrm{H}), 3.82(\mathrm{dq}, J=7.0,4.2 .0 .5 \mathrm{~Hz}, 1 \mathrm{H}), 3.37$ (ddt, $J=14.0,6.9,3.8 \mathrm{~Hz}$

$2 \mathrm{H}), 3.28-3.12(\mathrm{~m}, 2 \mathrm{H}), 2.25(\mathrm{~d}, J=1.8 \mathrm{~Hz}, 3 \mathrm{H}), 2.14-1.98(\mathrm{~m}, 1 \mathrm{H}), 1.91(\mathrm{~s}, 2 \mathrm{H}), 1.75-1.57(\mathrm{~m}, 2 \mathrm{H})$

$1.29-1.13(\mathrm{~m}, 4 \mathrm{H}), 0.89(\mathrm{t}, J=6.4 \mathrm{~Hz}, 6 \mathrm{H})$

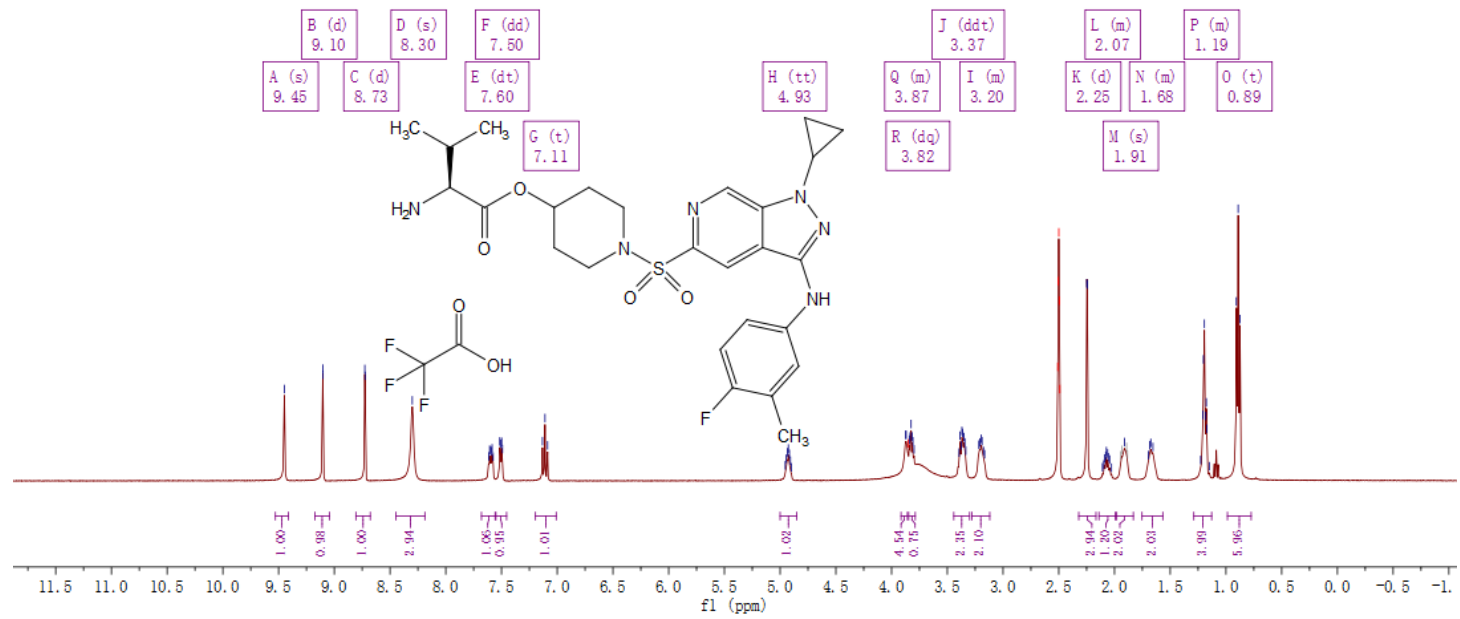

20191223-wct-1451-1-C/10

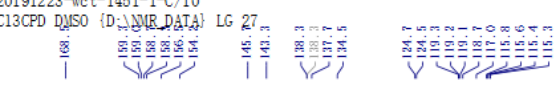

${ }^{13} \mathrm{C} \mathrm{NMR}\left(101 \mathrm{MHz}, \mathrm{DMSO}-d_{\mathrm{c}}\right) \delta 168.50,158.82(\mathrm{q}, J=32.5 \mathrm{~Hz}), 155.35(\mathrm{~d}, J=235.7 \mathrm{~Hz}), 145.67$,

$143.33,138.28,137.65,134.45,124.63(\mathrm{~d}, J=18.1 \mathrm{~Hz}), 119.27(\mathrm{~d}, J=4.1 \mathrm{~Hz}), 119.06,117.25(\mathrm{~d}, J=$

$297.5 \mathrm{~Hz}), 117.03,115.47(\mathrm{~d}, J=23.4 \mathrm{~Hz}), 115.28,70.68,57.70,43.55,40.42,30.18,29.93,29.86$,

$29.80,18.65,17.81,15.09(\mathrm{~d}, J=3.0 \mathrm{~Hz}), 6.93$.

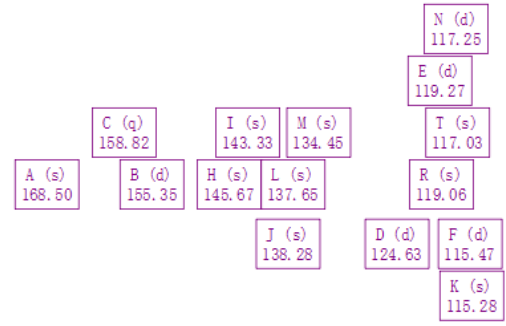<smiles>CC(=O)C(N)C(C)C</smiles>

i

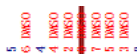

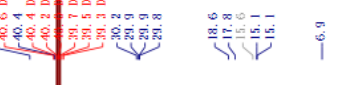

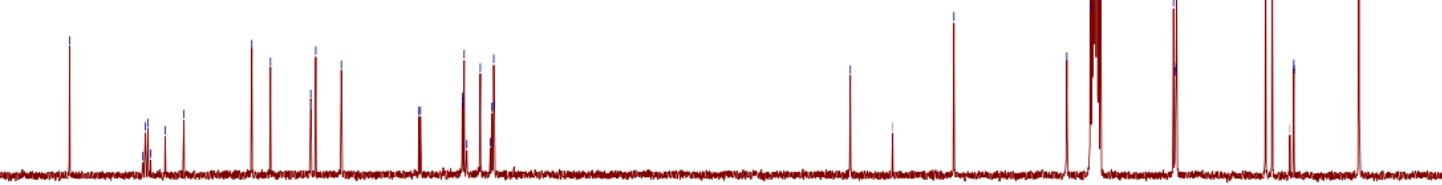




\section{Compound 63.}

\section{6 - $\mathrm{wct}-1453-1-\mathrm{H} / 10$

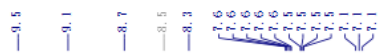

'H NMR (400 MHz, DMSO- $d_{\text {) }} \delta 9.45$ (s, 1H), 9.11 (s, $\left.1 \mathrm{H}\right), 8.73$ (s, $\left.1 \mathrm{H}\right), 8.28$ (s, 3H), 7.60 (dt, $J=7.7$, . $4 \mathrm{~Hz}, 1 \mathrm{H}), 7.50(\mathrm{dd}, J=6.9,2.8 \mathrm{~Hz}, 1 \mathrm{H}), 7.11(\mathrm{t}, J=9.2 \mathrm{~Hz}, 1 \mathrm{H}), 4.88(\mathrm{tt}, J=6.8,3.4 \mathrm{~Hz}, 1 \mathrm{H}), 4.06(\mathrm{~d}$ $J=7.0 \mathrm{~Hz}, 1 \mathrm{H}), 3.82(\mathrm{tt}, J=6.9,4.0 \mathrm{~Hz}, 1 \mathrm{H}), 3.53-3.25(\mathrm{~m}, 2 \mathrm{H}), 3.28-3.0 \mathrm{~s}(\mathrm{~m}, 2 \mathrm{H}), 2.25(\mathrm{~s}, 3 \mathrm{H}), 1.89$

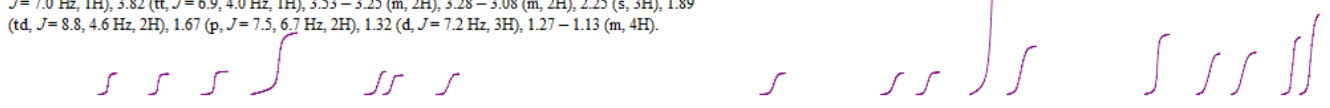

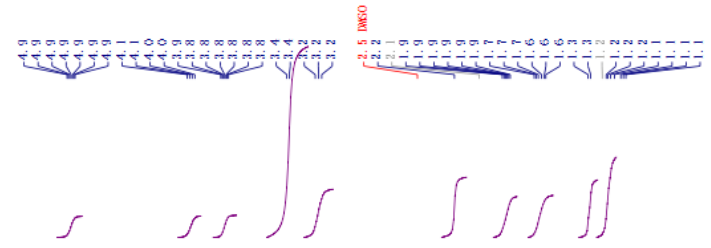

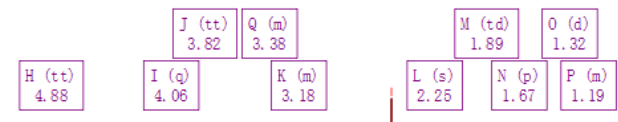
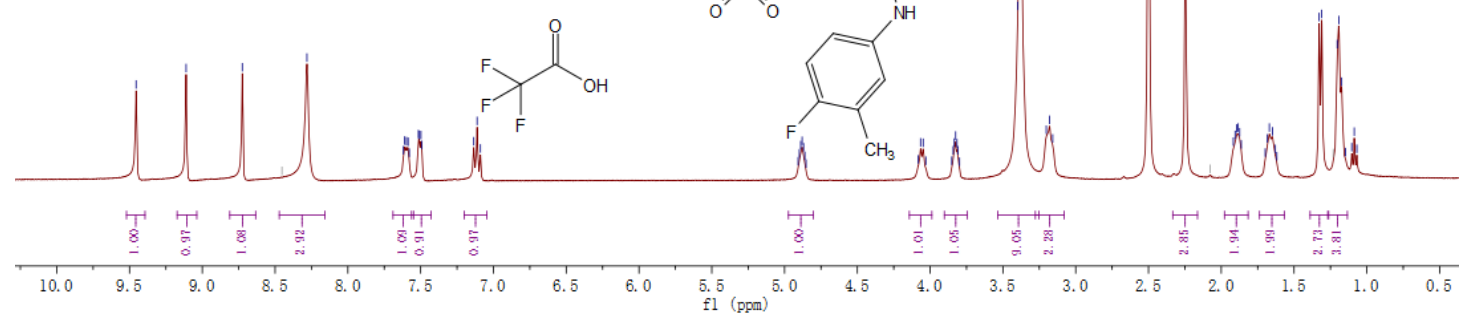

20191223-wct-1453-1-C/10
C13CPD DUSO \{D: $\backslash$ NIR_DATA\} LG 28

$\overbrace{}^{2}$<smiles>CNc1nn(C2CC2)c2cnc(S(=O)(=O)N3CCC(OC(C)=O)CC3)cc12</smiles>

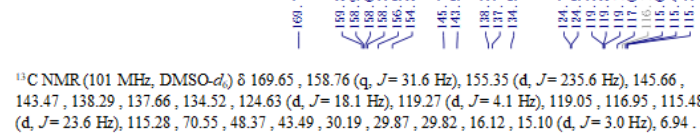<smiles>CCNc1nn(C2CC2)c2cnc(S(=O)(=O)N3CCC(OC(=O)C(C)N)CC3)cc12</smiles>

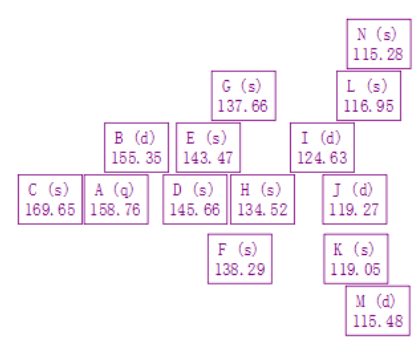

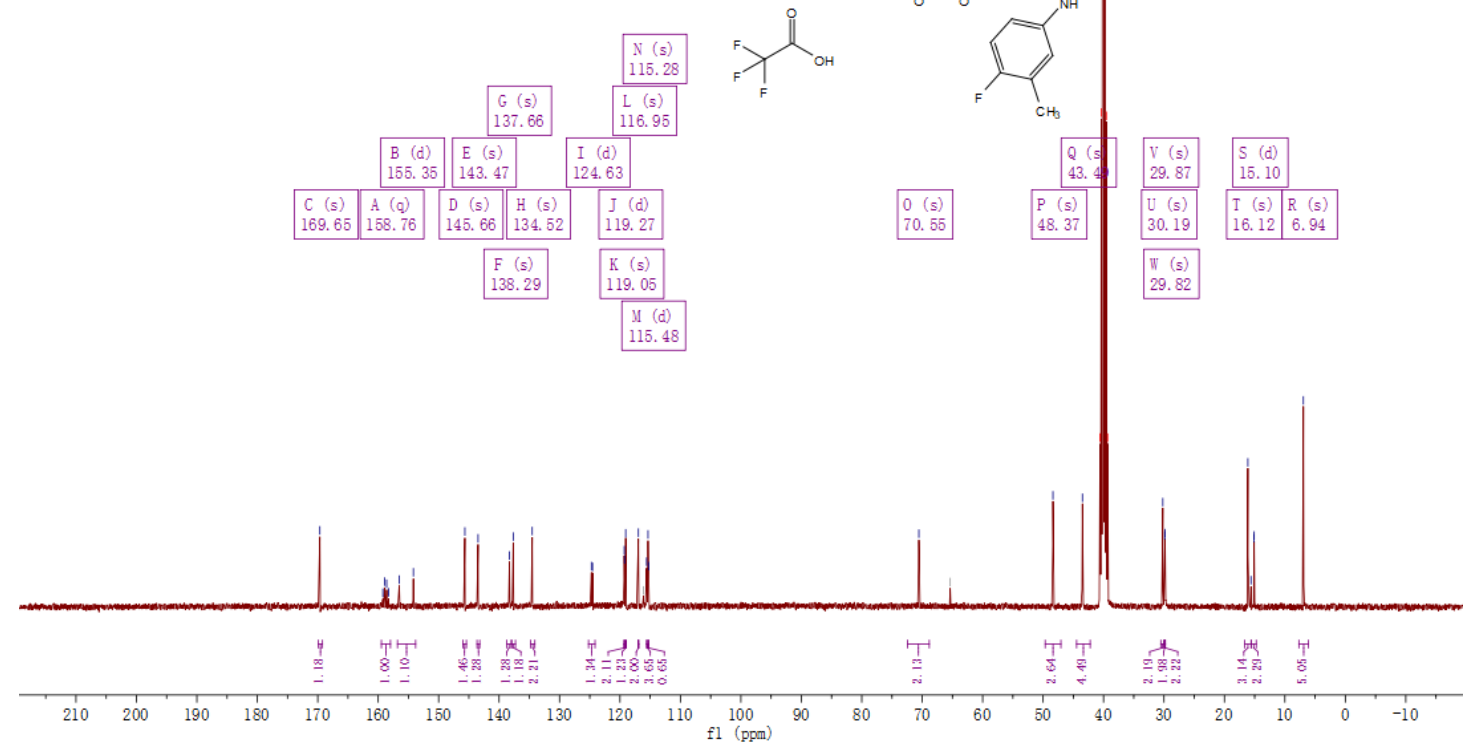

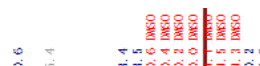

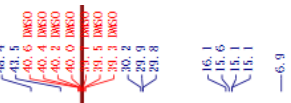




\section{Compound 64.}

191106 -wct-1454-1-H/10

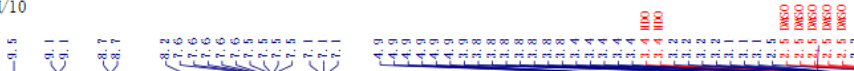

$(\mathrm{s}, 3 \mathrm{H}), 7.61(\mathrm{dt}, J=8.7,3.6 \mathrm{~Hz}, 1 \mathrm{H}), 7.31(\mathrm{dd}, J=6.9,2.8 \mathrm{~Hz}, 1 \mathrm{H}), 7.12(\mathrm{t}, J=9.2 \mathrm{~Hz}, 1 \mathrm{H}), 4.90$ (td, $J$

$12.3,83,3.7 \mathrm{~Hz}, 2 \mathrm{HH}, 225(\mathrm{~d}, J=1.9 \mathrm{~Hz}, 3 \mathrm{H}), 1.98-1.85(\mathrm{~m}, 2 \mathrm{H}), 1.65(\mathrm{dtd}, J=12.1,7.3,6 \mathrm{~Hz}, 2 \mathrm{H})$

1.21 (dddd, $J=12.0,9.5,6.7,5.0 \mathrm{~Hz}, 4 \mathrm{H})$.

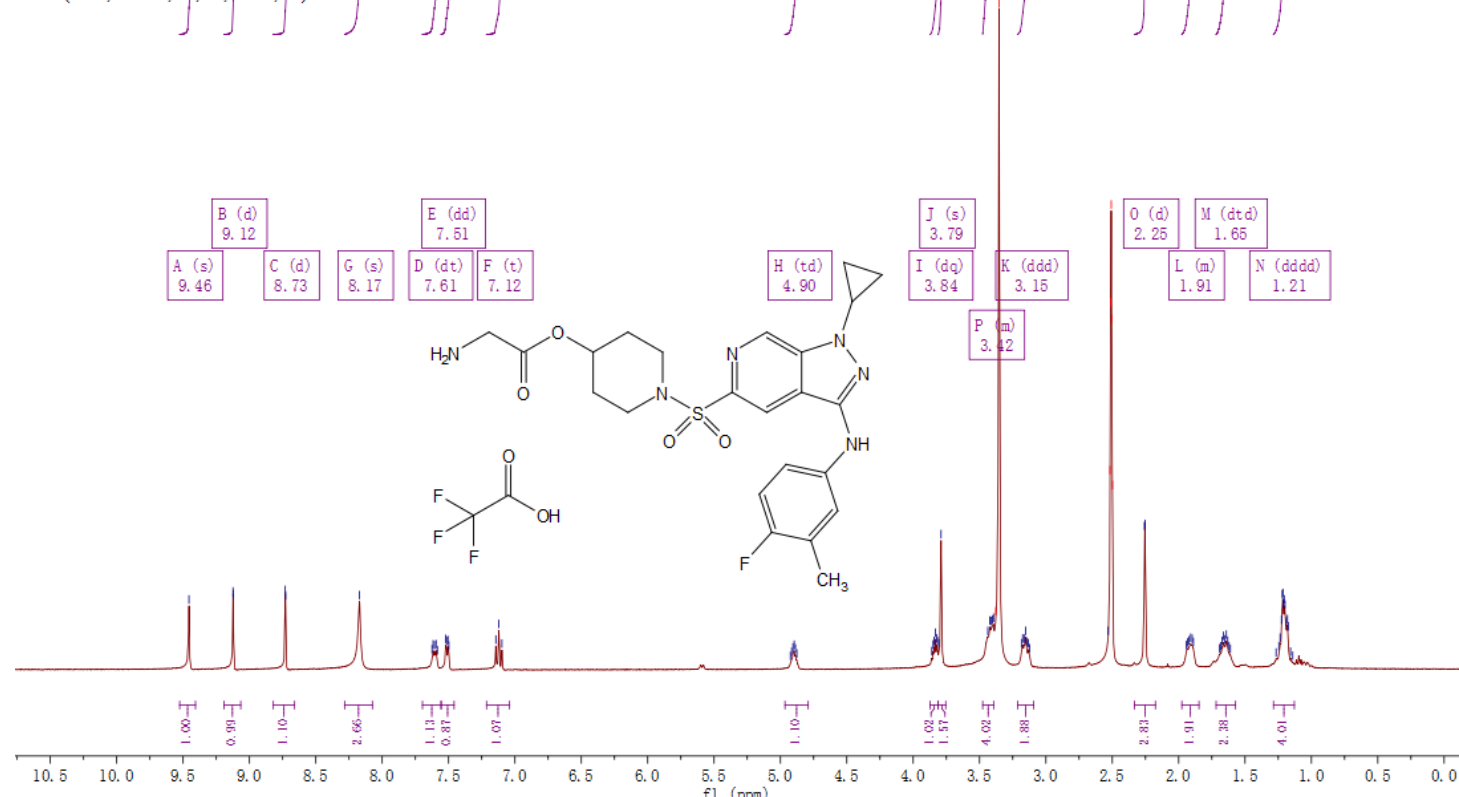

20191223-wct-1454-1-C/10
C13CPD DUSO $\{$ D:dWUR_DATA\} LLE 29

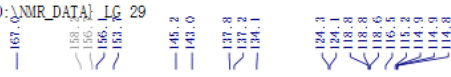

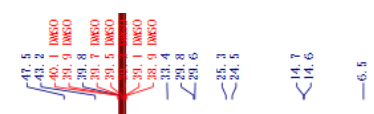
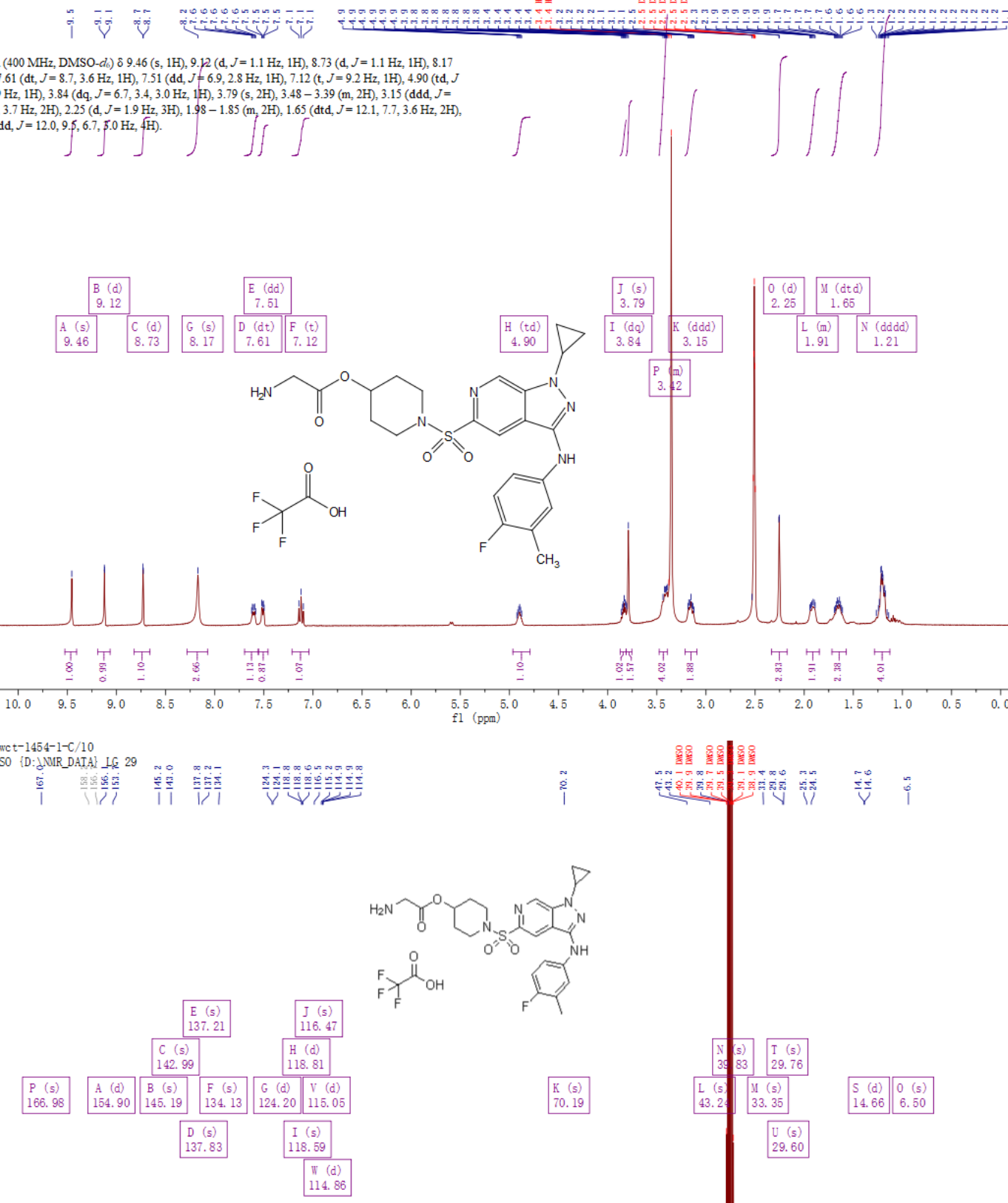


\section{Compound 65.}

20191106-wct-1455-0-H/10
PROTON CDC13 \{D: LNIR_DATA\} LG 56 Oig

요ㄹㅛㅛ 을

(dd, $J=6.6,30 \mathrm{MHz}$, Chloroform- $d$ ) $\delta 8.43$ (dd, $J=4.0,1.1 \mathrm{~Hz}, 1 \mathrm{H}), 7.45$ (dt, $J=8.9,3.6 \mathrm{~Hz}, 1 \mathrm{H}$ ), 7.39

(dd, $J=6.6,3.0 \mathrm{~Hz}, 1 \mathrm{H}), 6.98(\mathrm{t}, J=9.0 \mathrm{~Hz}, 1 \mathrm{H}), 6.95(\mathrm{~s}, 1 \mathrm{H}), 4.86(\mathrm{tt}, J=7.9,3.8 \mathrm{~Hz}, 1 \mathrm{H}), 3.63(\mathrm{tq}, \mathrm{J}=$ $11.6,3.5 \mathrm{~Hz}, 3 \mathrm{H}), 3.18(\mathrm{ddd}, J=12.4,8.6,3.5$
$2 \mathrm{H}), 1.77-1.68(\mathrm{~m}, 2 \mathrm{H}), 1.33-1.21(\mathrm{~m}, 4 \mathrm{H})$.<smiles>CN(C)CC(=O)OC1CCCCC1</smiles><smiles>C1CN2CC23CC2CC23C1</smiles>

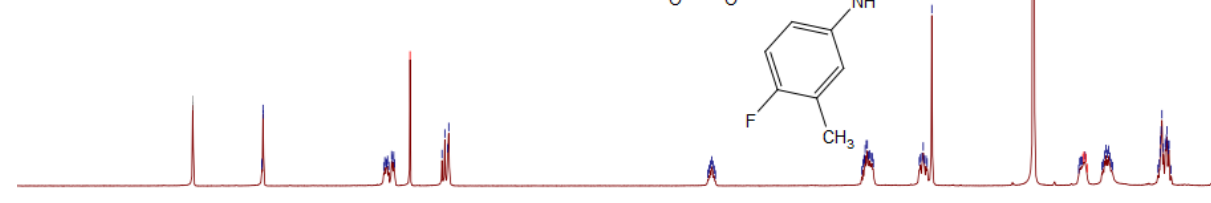

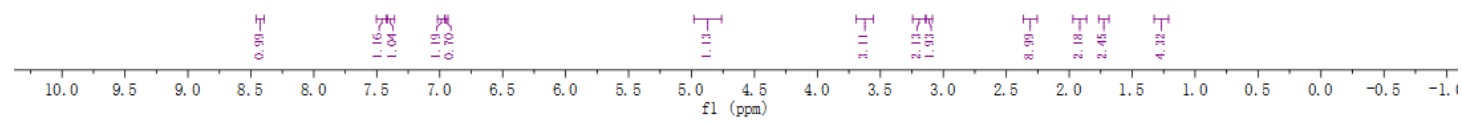

$20200105-w c t-1455-0-C / 10$
C13CPD CDC13 [D: WNIR_DATA\} LG 25

l l

${ }^{13} \mathrm{CNMR}(101 \mathrm{MHz}$, Chloroform- $d$ ) $8169.75,156.32$ (d, $J=238.4 \mathrm{~Hz}), 145.63,143.61,137.82$,

$136.93(\mathrm{~d}, J=2.5 \mathrm{~Hz}), 133.72,125.19(\mathrm{~d}, J=18.4 \mathrm{~Hz}), 119.96(\mathrm{~d}, J=4.4 \mathrm{~Hz}), 119.41,116.19,115.69$

(d, $J=7$.

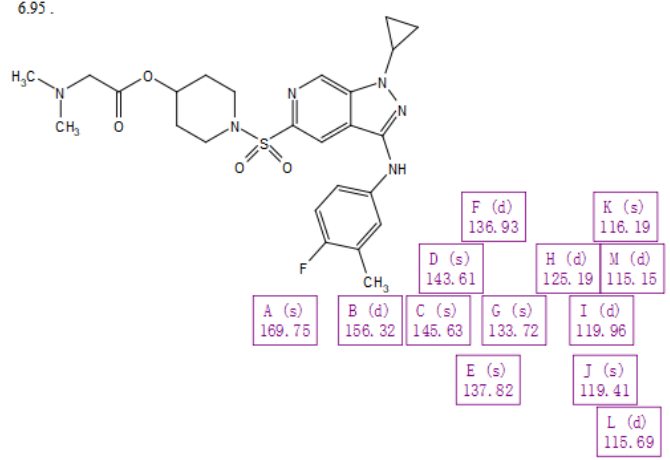<smiles>Nc1[nH]c2cnc(C=O)cc2c1N</smiles><smiles>C1CCCC1</smiles>

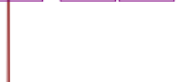


Compound 66.

2019101 G-wct-1455-1-H/10

'H NMR ( $400 \mathrm{MHz}$, DMSO- $\left.d_{\mathrm{s}}\right) \delta 11.93(\mathrm{~s}, 2 \mathrm{H}), 9.44(\mathrm{~d}, J=3.2 \mathrm{~Hz}, 1 \mathrm{H}), 9.12(\mathrm{~d}, J=3.2 \mathrm{~Hz}, 1 \mathrm{H}), 8.71$ $(\mathrm{d}, J=3.2 \mathrm{~Hz}, 1 \mathrm{H}), 7.60$ (dd, $J=8.6,4.3 \mathrm{~Hz}, 1 \mathrm{H}), 7.55-7.44(\mathrm{~m}, 1 \mathrm{H}), 7.11$ (td, $J=9.3,3.3 \mathrm{~Hz}, 1 \mathrm{H})$, $4.78(\mathrm{~s}, 1 \mathrm{H}), 3.82(\mathrm{dq}, J=7.5,3.8 \mathrm{~Hz}, 1 \mathrm{H}), 3.21-3.00(\mathrm{~m}, 4 \mathrm{H}), 2.25(\mathrm{~s}, 3 \mathrm{H}), 2.20(\mathrm{~d}, J=32 \mathrm{~Hz}, 6 \mathrm{H})$ $1.98-1.78(\mathrm{~m}, 5 \mathrm{H}), 1.60(\mathrm{~s}, 2 \mathrm{H}), 1.19(\mathrm{t}, J=7.6 \mathrm{~Hz}, 4 \mathrm{H}) . \iiint \iint$

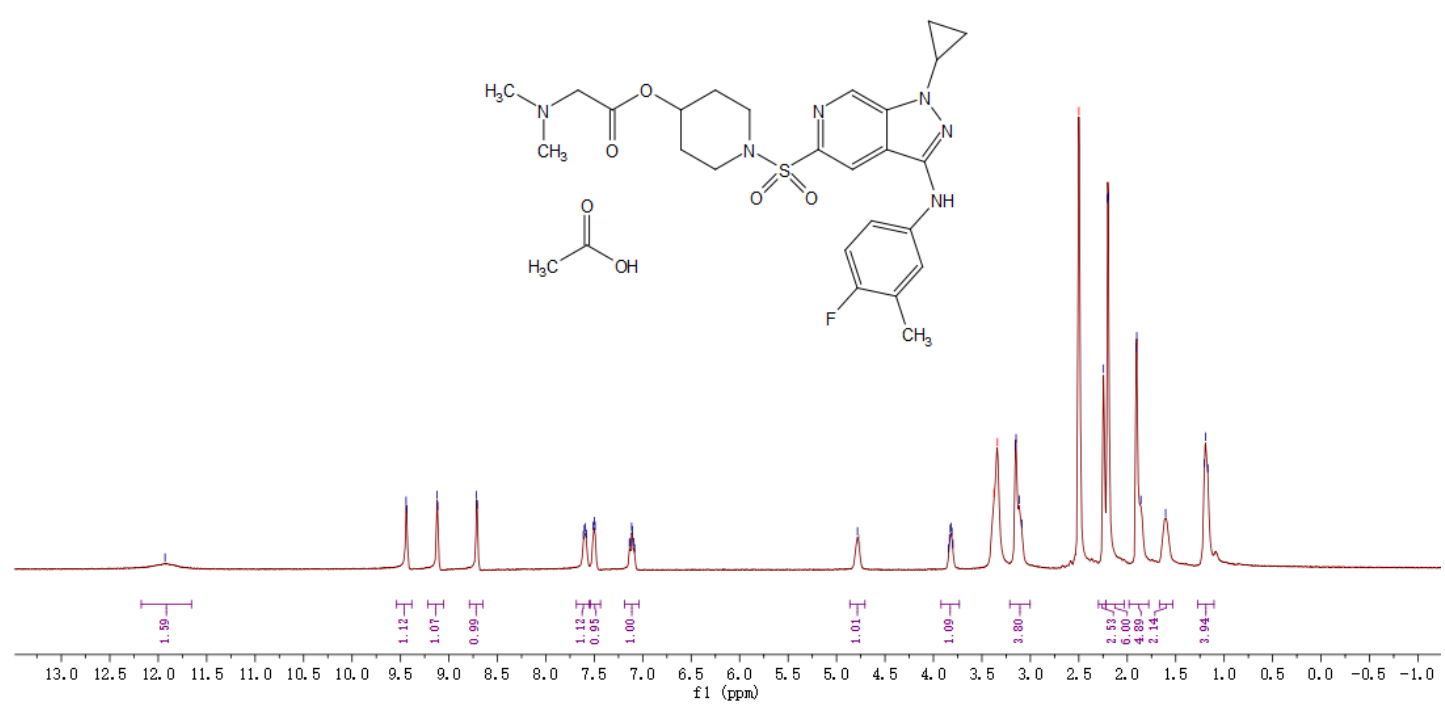

\section{Compound 67.}

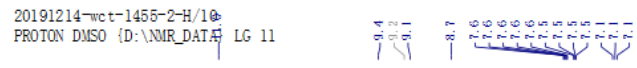

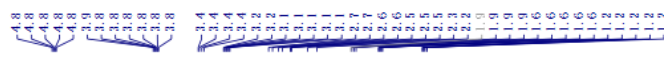

H NMR (400 MHz, DMSO- $\left.d_{\mathrm{s}}\right) \delta 10.99$ (s, $2 \mathrm{H}$ ), $9.43(\mathrm{~s}, 1 \mathrm{H}), 9.12(\mathrm{~s}, 1 \mathrm{H}), 8.71$ (s, $1 \mathrm{H}$ ), 7.60 (dd, $J=$ $8.3,4.1 \mathrm{~Hz}, 1 \mathrm{H}$ ), 7.50 (dd, $J=6.8,2.8 \mathrm{~Hz}, 1 \mathrm{H}$ ), $7.11(\mathrm{t}, J=9.2 \mathrm{~Hz}, 1 \mathrm{H}), 4.82$ (dt, $J=8.0,4.4 \mathrm{~Hz}, 1 \mathrm{H}$ ), 源

$1.24-1.09(\mathrm{~m}, 4 \mathrm{H})$.

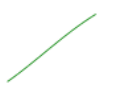

$\iiint$

$\iiint$<smiles>CN(C)CC(=O)OC1CCCCC1</smiles><smiles>CCN(C)S(=O)(=O)c1cc2c(C)nn(C3CC3)c2c2c1CCN2C1CC1</smiles>

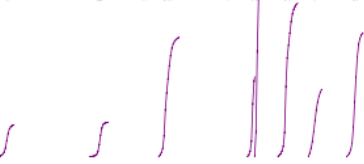




\section{Compound 68.}

20200113-wct-1455-3-H/10
PROTON DUSO [D: INR_DATA
LG 18

'H NMR (400 MHz, DMSO-d $\left.d_{s}\right) 89.44(\mathrm{~s}, 1 \mathrm{H}), 9.45(\mathrm{~s}, 1 \mathrm{H}), 9.12(\mathrm{~s}, 1 \mathrm{H}), 8.73(\mathrm{~s}, 1 \mathrm{H}), 7.68-7.24(\mathrm{~m}$, $3 \mathrm{H}), 7.11(\mathrm{t}, J=9.2 \mathrm{~Hz}, 1 \mathrm{H}), 5.01-4.84(\mathrm{~m}, 1 \mathrm{H}), 4.15(\mathrm{~d}, J=3.8 \mathrm{~Hz}, 2 \mathrm{H}), 3.83(\mathrm{tt}, J=7.0,4.0 \mathrm{~Hz}, 1 \mathrm{H})$,
$3.38(\mathrm{q}, J=7.2,6.8 \mathrm{~Hz}, 2 \mathrm{H}), 3.17(\mathrm{t}, J=9.2 \mathrm{~Hz}, 2 \mathrm{H}), 2.80(\mathrm{~d}, J=3.3 \mathrm{~Hz}, 6 \mathrm{H}), 2.35(\mathrm{~s}, 6 \mathrm{H}), 2.25(\mathrm{~s}, 3 \mathrm{H})$, $1.92(\mathrm{t}, J=9.9 \mathrm{~Hz}, 2 \mathrm{H}), 1.69(\mathrm{~s}, 2 \mathrm{H}), 1.27-1.13(\mathrm{~m}, 4 \mathrm{H})$
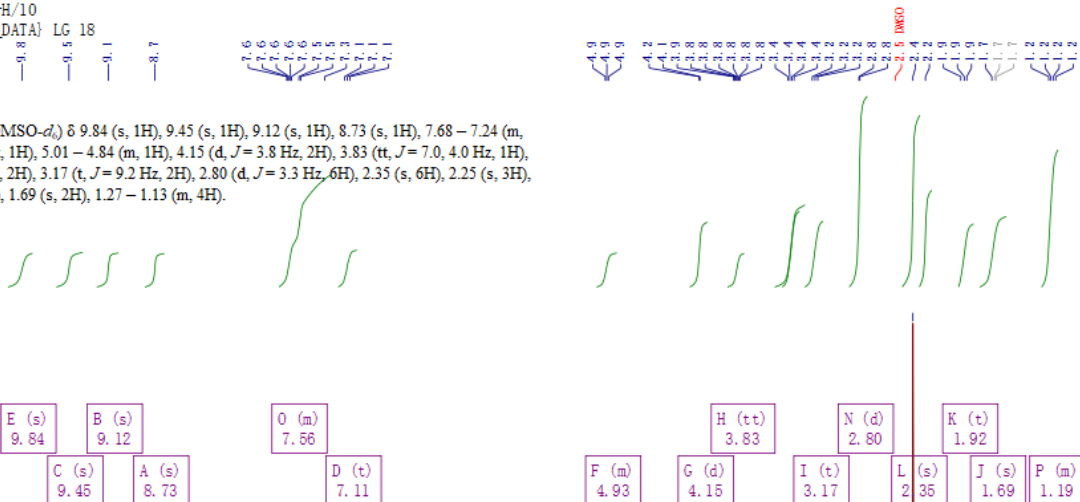

\section{0 (m)
7.56}
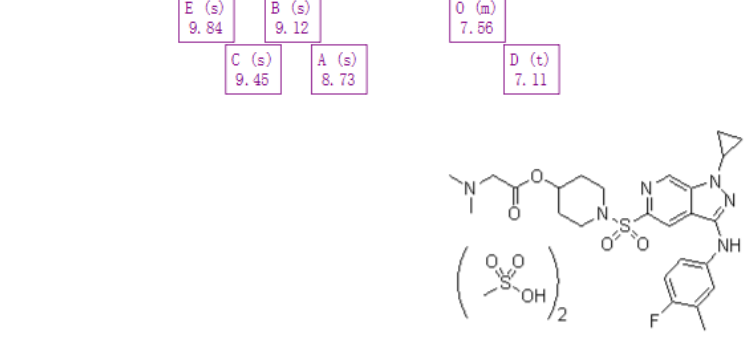

\section{F (II)
4.93}
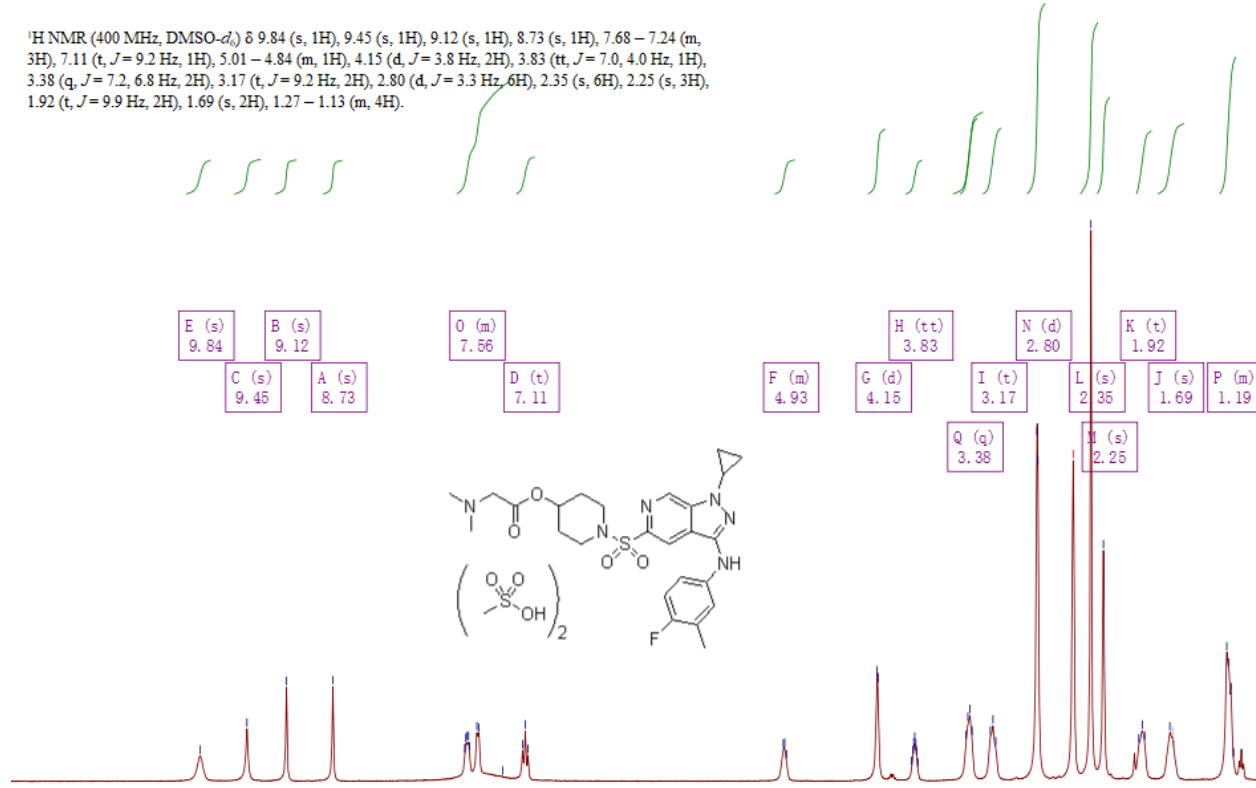

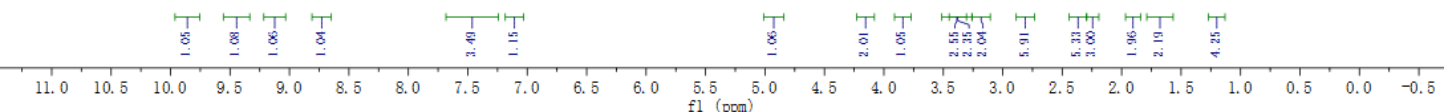

\section{Compound 69.}

191018-wct-1455-4-H/10

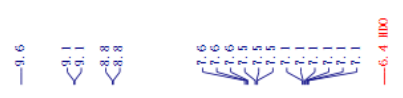

'H NMR ( $400 \mathrm{MHz}$, DMSO- $\left.d_{\mathrm{d}}\right) \delta 10.55(\mathrm{~s}, 1 \mathrm{H}), 9.59(\mathrm{~s}, 1 \mathrm{H}), 9.12(\mathrm{~d}, J=3.3 \mathrm{~Hz}, 1 \mathrm{H}), 8.79(\mathrm{~d}, J=3$. $\mathrm{Hz}, 1 \mathrm{H}), 7.69-7.58(\mathrm{~m}, 1 \mathrm{H}), 7.58-7.49(\mathrm{~m}, 1 \mathrm{H}), 7.11(\mathrm{td}, J=9.4,3.4 \mathrm{~Hz}, 1 \mathrm{H}), 4.93(\mathrm{~s}, 1 \mathrm{H}), 4.15(\mathrm{~s}$, $2 \mathrm{H}), 3.90-3.77(\mathrm{~m}, 1 \mathrm{H}), 3.39(\mathrm{t}, J=9.6 \mathrm{~Hz}, 2 \mathrm{H}), 3.19(\mathrm{t}, J=9.6 \mathrm{~Hz}, 2 \mathrm{H}), 2.79(\mathrm{~s}, 6 \mathrm{H}), 2.25(\mathrm{~s}, 3 \mathrm{H}), 1.93$ (d, $J=12.8 \mathrm{~Hz}, 2 \mathrm{H}), 1.68(\mathrm{~d}, J=11.3 \mathrm{~Hz}, 2 \mathrm{H}), 1.27-1.13(\mathrm{~m}, 4 \mathrm{H})$
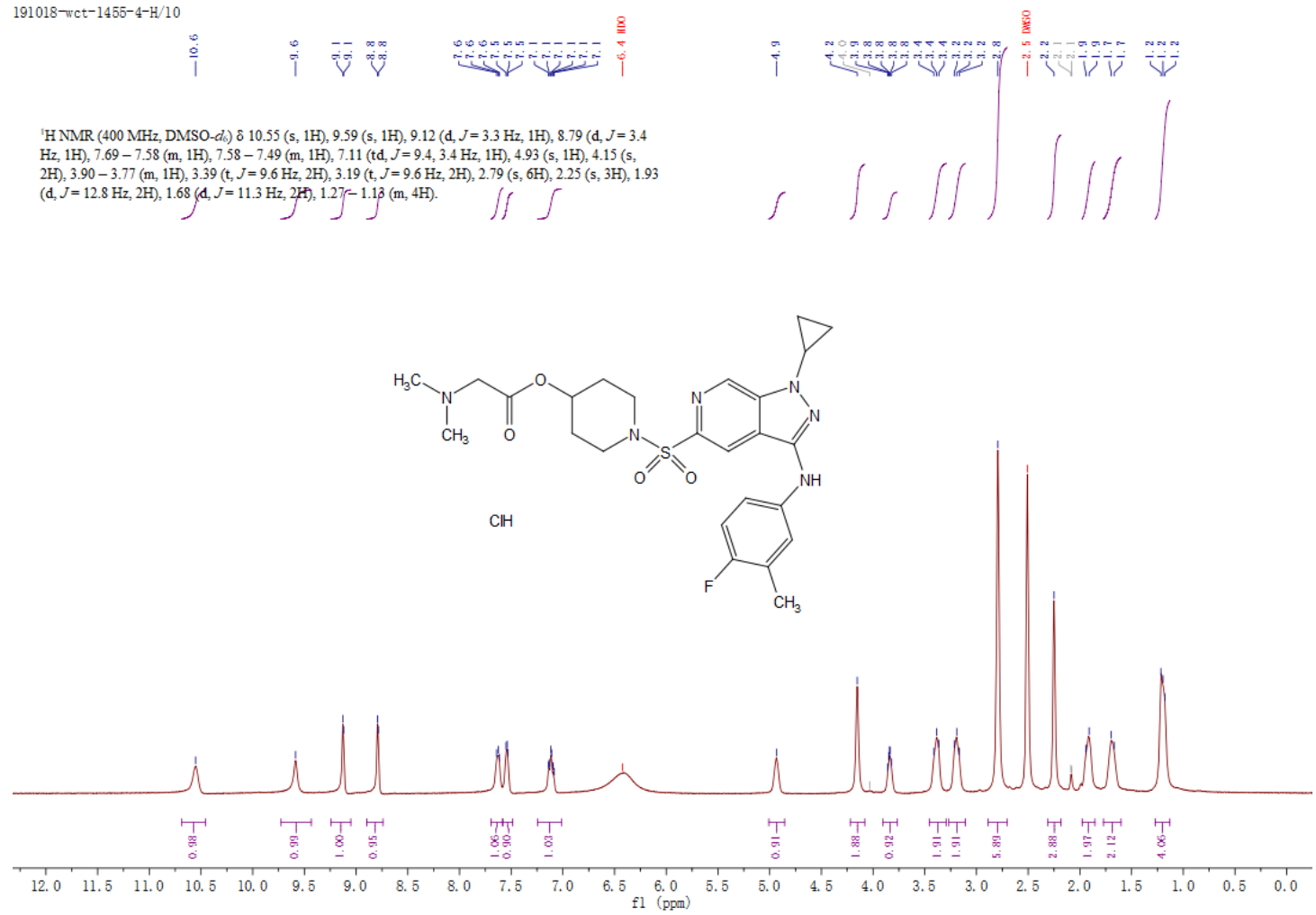


\section{Compound 70.}

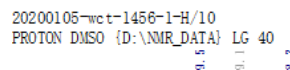

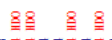

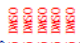

然

'H NMR (400 MHz, DMSO-d $)$ ) $9.45(\mathrm{~s}, 1 \mathrm{H}), 8.74(\mathrm{~s}, 1 \mathrm{H}), 7.61$ (dt, $J=8.9,3.5 \mathrm{~Hz}, 1 \mathrm{H}), 7.51$ (dd, $J=$

$6.8,2.8 \mathrm{~Hz}, 1 \mathrm{H}), 7.12(\mathrm{t}, J=9.2 \mathrm{~Hz}, 1 \mathrm{H}), 6.06(\mathrm{~s}, 2 \mathrm{H}), 4.92(\mathrm{td}, J=7.8,4.0 \mathrm{~Hz}, 1 \mathrm{H}), 4.00(\mathrm{~s}, 2 \mathrm{H}), 3.84$
(tt, $J=6.9,3.9 \mathrm{~Hz}, 1 \mathrm{H}), 3.39(\mathrm{t}, J=7.8 \mathrm{~Hz}, 3 \mathrm{H}), 3.18(\mathrm{ddd}, J=12.2,8.2,3.7 \mathrm{~Hz}, 2 \mathrm{H}), 2.72(\mathrm{~s}, 6 \mathrm{H}), 2.39$

$-2.18(\mathrm{~m}, 3 \mathrm{H}), 1.98-1.82(\mathrm{~m}, 2 \mathrm{H}), 1.68(\mathrm{qd}, J=8.1,4.0 \mathrm{~Hz}, 2 \mathrm{H}), 1.34-1.16(\mathrm{~m}, 4 \mathrm{H})$.
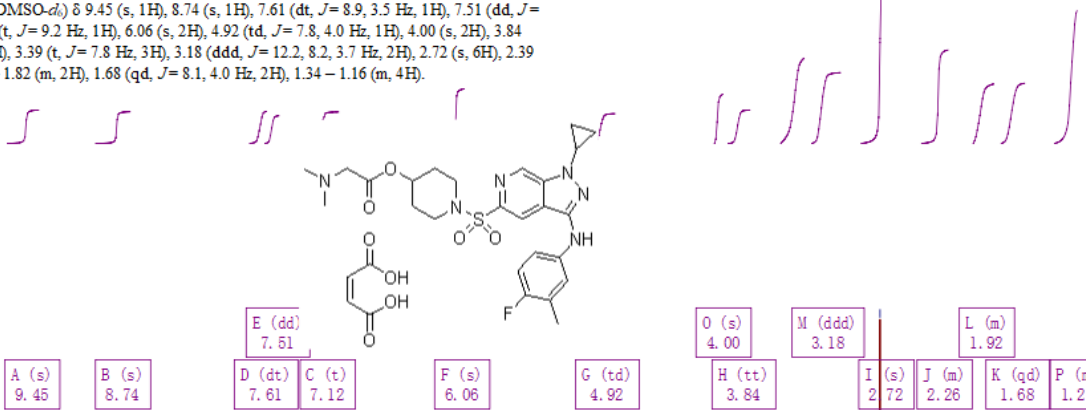

\begin{tabular}{|c|c|c|c|c|c|}
\hline$A(s)$ & $B(s)$ & $D(d t)$ & $C(t)$ & $F(s)$ & $G(t d)$ \\
9.45 & 7.61 & 7.12 & 6.06 & 4.92 \\
\hline
\end{tabular}

\begin{tabular}{|c|c|c|c|c|c|}
\hline $\begin{array}{ll}0(s) \\
4.00 \\
\end{array}$ & \begin{tabular}{|c|} 
M (ddd) \\
3.18 \\
\end{tabular} & & & $\begin{array}{l}\text { L (m) } \\
1.92\end{array}$ & \\
\hline $\begin{array}{c}\mathrm{H}(\mathrm{tt}) \\
3.84\end{array}$ & & $\begin{array}{ll}\text { I } & (\mathrm{s} . \\
2 & 72\end{array}$ & \begin{tabular}{|l} 
J (m) \\
2.26
\end{tabular} & $\begin{array}{c}\mathrm{K}(\mathrm{gd}) \\
1.68\end{array}$ & $\begin{array}{l}\mathrm{P} \text { (m) } \\
1.21\end{array}$ \\
\hline
\end{tabular}

N ( $(\mathrm{t})$
3. 39

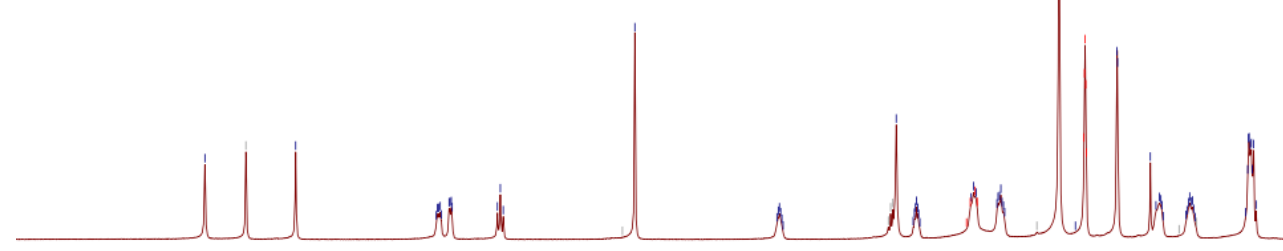

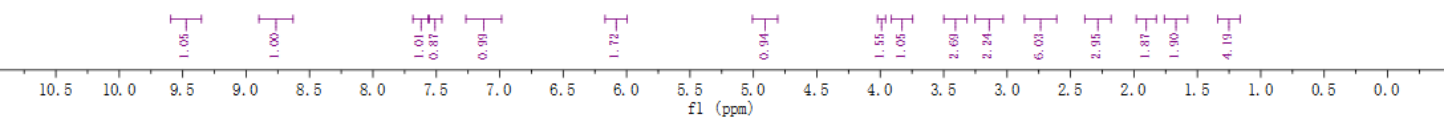


Supplementary Table S2: Purity of the tested compounds.

\begin{tabular}{|c|c|c|c|c|c|}
\hline Compd. & $t_{R}(\min )$ & Purity $(\%)$ & Compd. & $t_{R}(\min )$ & Purity (\%) \\
\hline 1 & 7.885 & 96.41 & 32 & 11.032 & 98.14 \\
\hline 2 & 9.757 & 98.88 & 33 & 11.214 & 98.54 \\
\hline 3 & 10.960 & 96.52 & 34 & $1230-2$ & 97.86 \\
\hline 4 & 9.828 & 99.89 & 35 & 9.681 & 98.07 \\
\hline 5 & 10.163 & 99.62 & 36 & 9.396 & 98.85 \\
\hline 6 & 11.062 & 96.08 & 37 & 9.993 & 98.37 \\
\hline 7 & 12.032 & 97.96 & 38 & 11.826 & 96.76 \\
\hline 8 & 11.422 & 98.94 & 39 & 10.760 & 95.88 \\
\hline 9 & 11.098 & 95.55 & 40 & 11.94 & 95.13 \\
\hline 10 & 11.564 & 98.14 & 41 & 12.233 & 99.58 \\
\hline 11 & 9.805 & 95.58 & 42 & 10.896 & 98.36 \\
\hline 12 & 10.306 & 95.90 & 43 & 11.796 & 98.81 \\
\hline 13 & 10.311 & 99.79 & 44 & 8.983 & 98.39 \\
\hline 14 & 10.133 & 100.00 & 45 & 10.684 & 98.56 \\
\hline 15 & 10.758 & 99.77 & 46 & 12.745 & 98.22 \\
\hline 16 & 9.321 & 99.28 & 47 & 11.349 & 98.99 \\
\hline 17 & 9.221 & 98.90 & 48 & 11.762 & 90.66 \\
\hline 18 & 10.180 & 99.40 & 49 & 11.226 & 96.39 \\
\hline 19 & 10.241 & 99.00 & 50 & 11.483 & 98.73 \\
\hline 20 & 10.338 & 97.76 & 51 & 10.846 & 97.80 \\
\hline $21^{a}$ & 1.55 & 95.85 & 52 & 11.519 & 98.26 \\
\hline 22 & 9.553 & 98.20 & 53 & 10.189 & 98.09 \\
\hline 23 & 7.600 & 98.72 & 54 & 11.297 & 97.18 \\
\hline 24 & 7.773 & 99.24 & 55 & 13.265 & 97.60 \\
\hline 25 & 8.264 & 97.99 & 56 & 9.158 & 98.97 \\
\hline 26 & 8.502 & 98.44 & 57 & 10.058 & 98.94 \\
\hline 27 & 9.272 & 99.00 & 58 & 11.538 & 97.48 \\
\hline 28 & 8.299 & 97.99 & 59 & 8.875 & 98.85 \\
\hline 29 & 7.650 & 97.98 & 60 & 10.414 & 97.95 \\
\hline 30 & 9.326 & 97.99 & 67 & 7.240 & 99.60 \\
\hline 31 & 10.290 & 98.32 & & & \\
\hline
\end{tabular}




\section{HPLC spectrum}

1

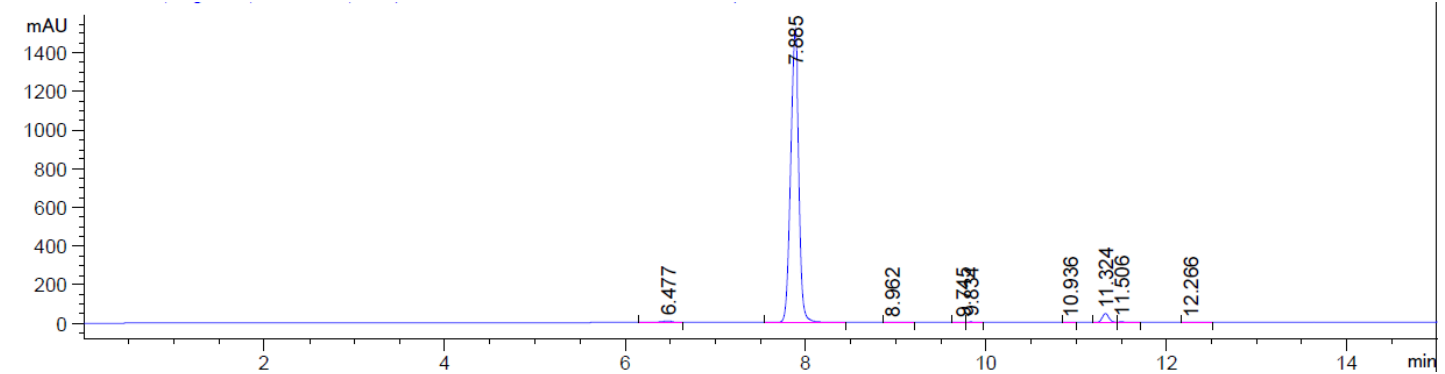

2

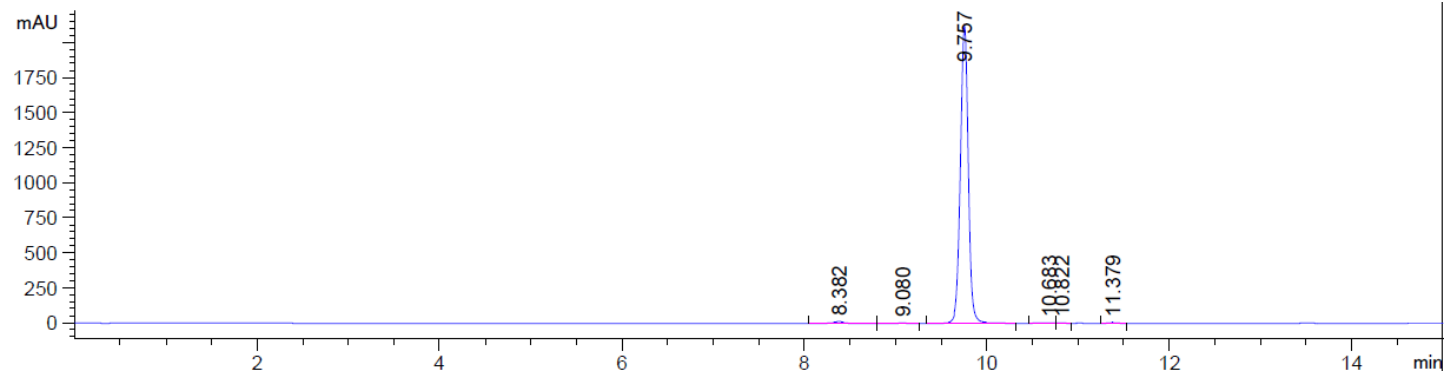

3

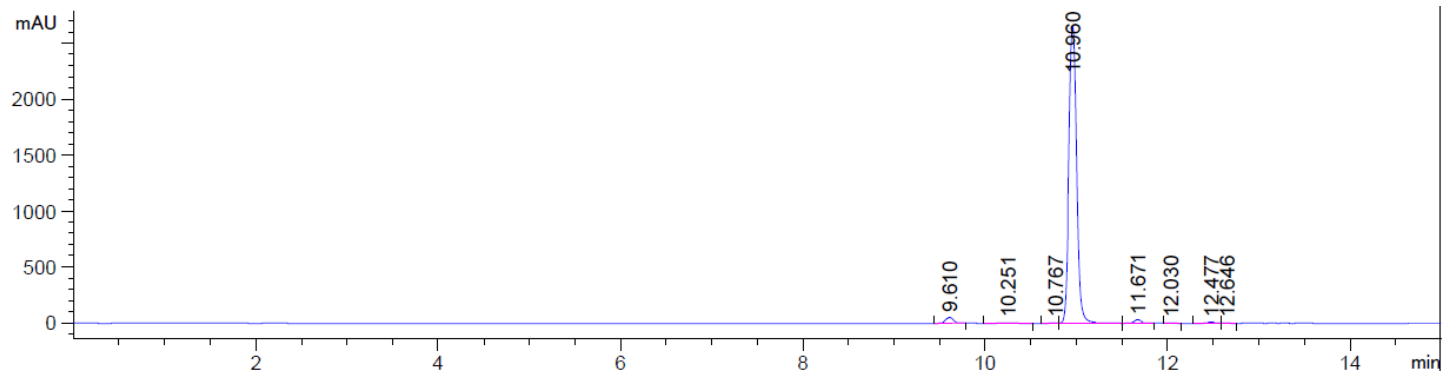

4

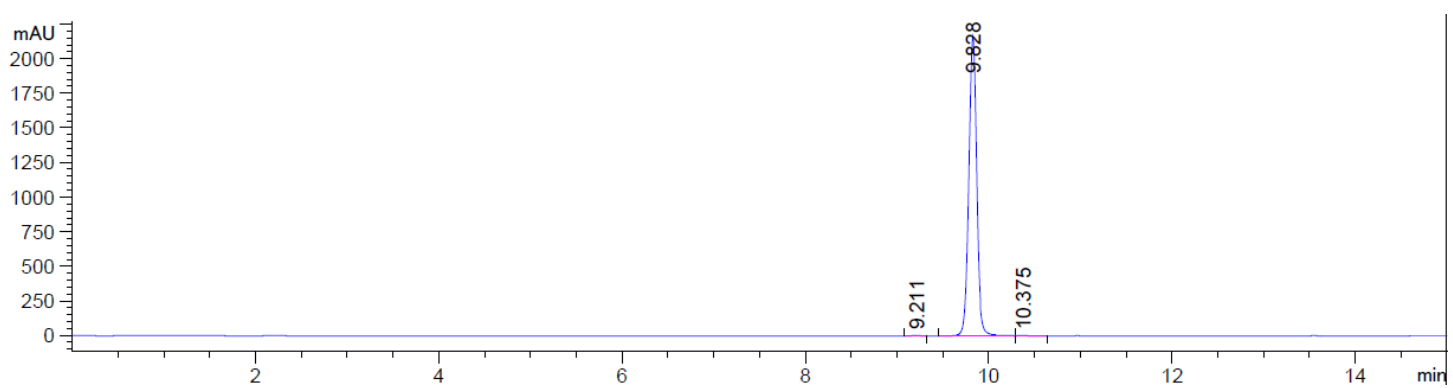

5

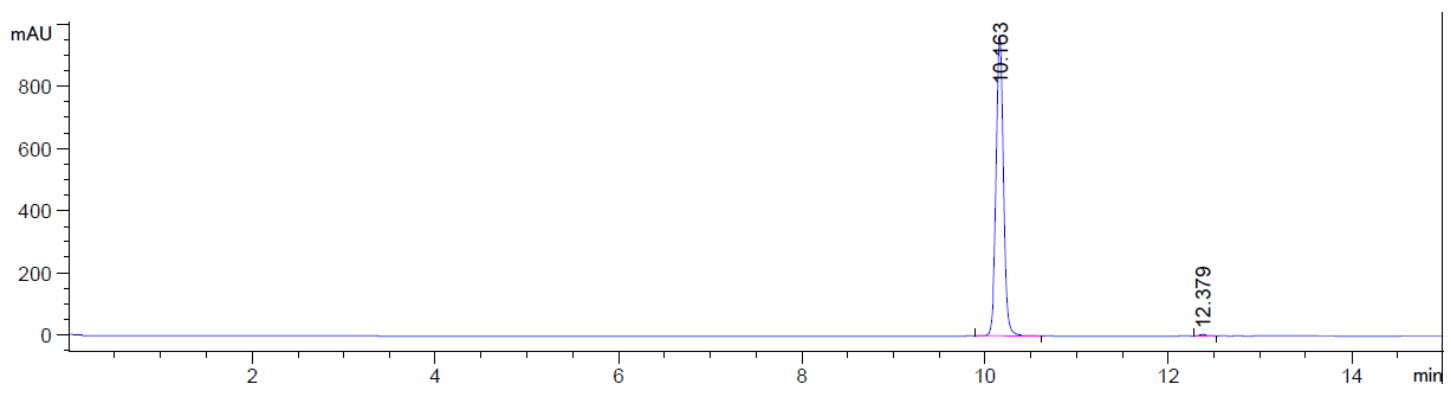




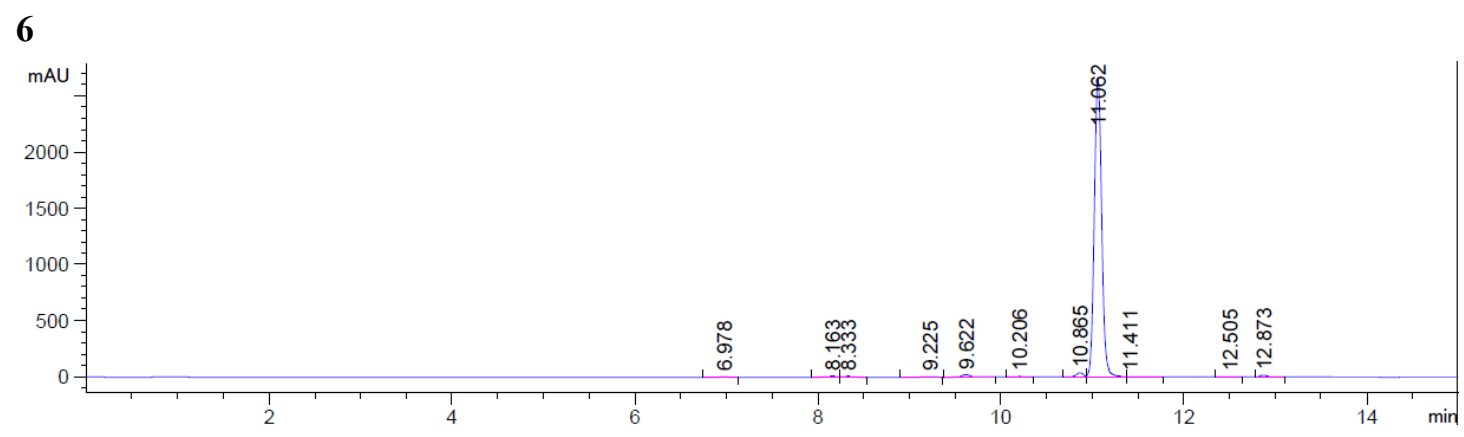

7

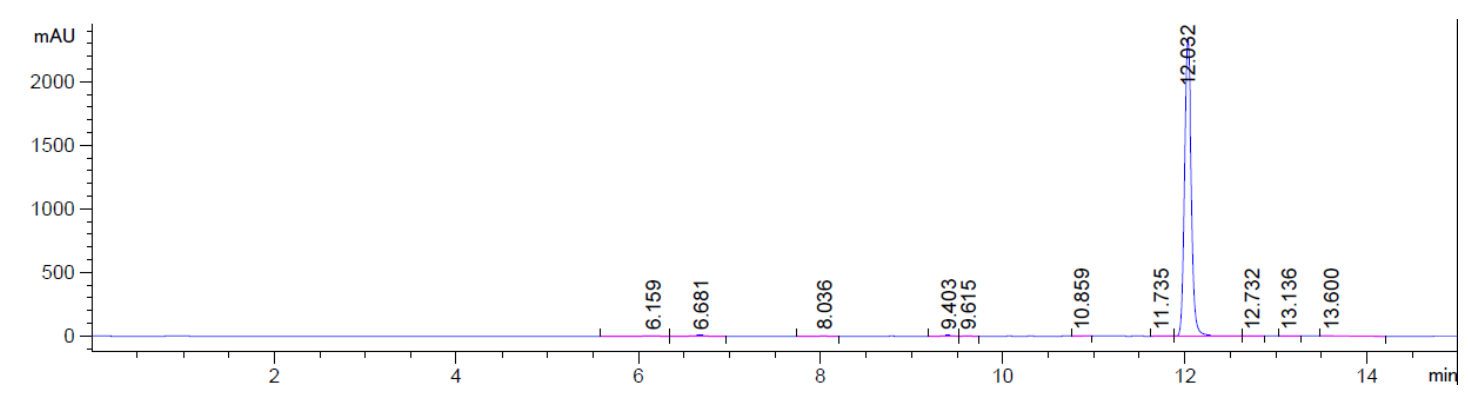

8

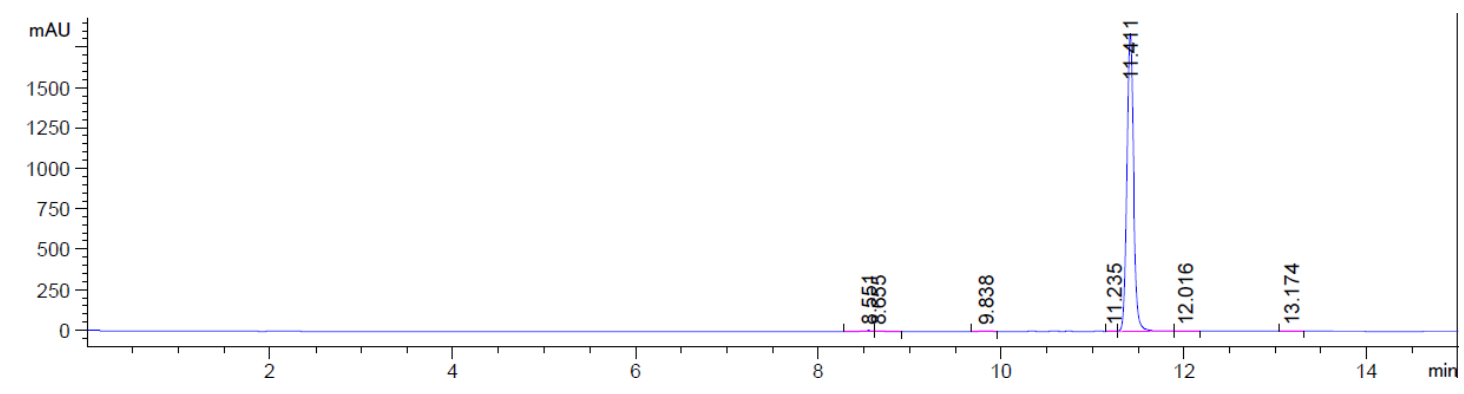

9

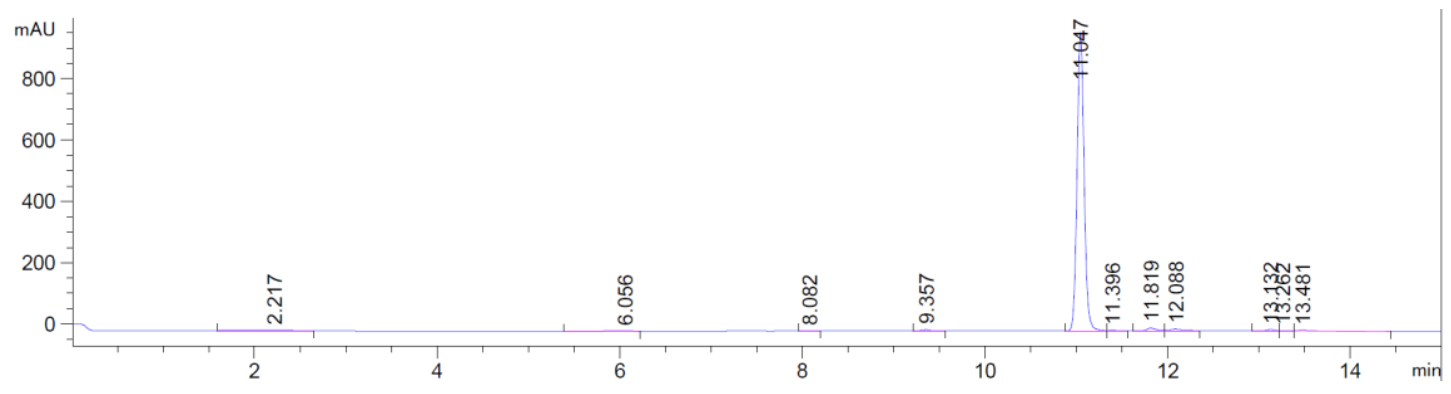

10

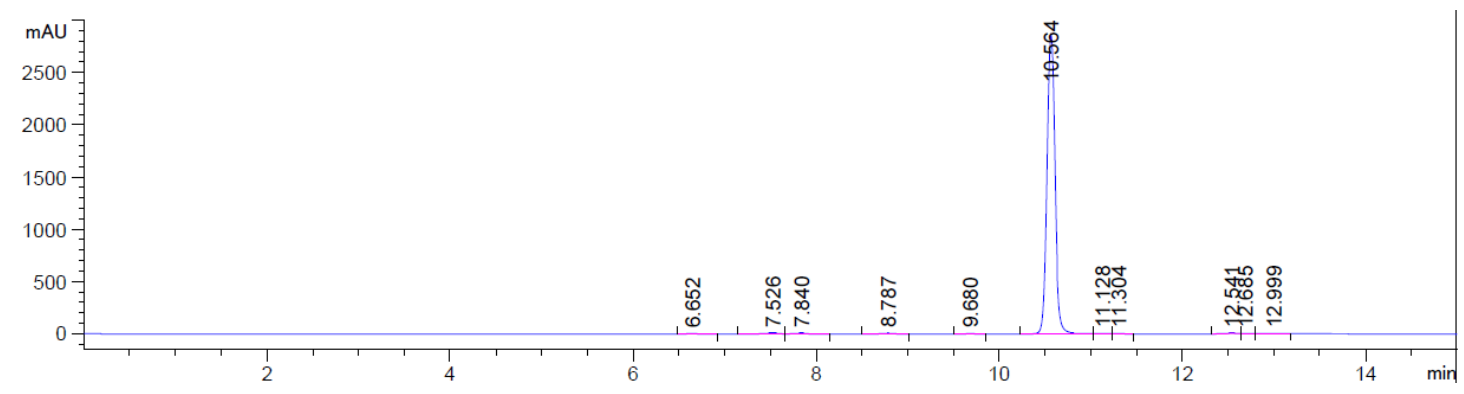




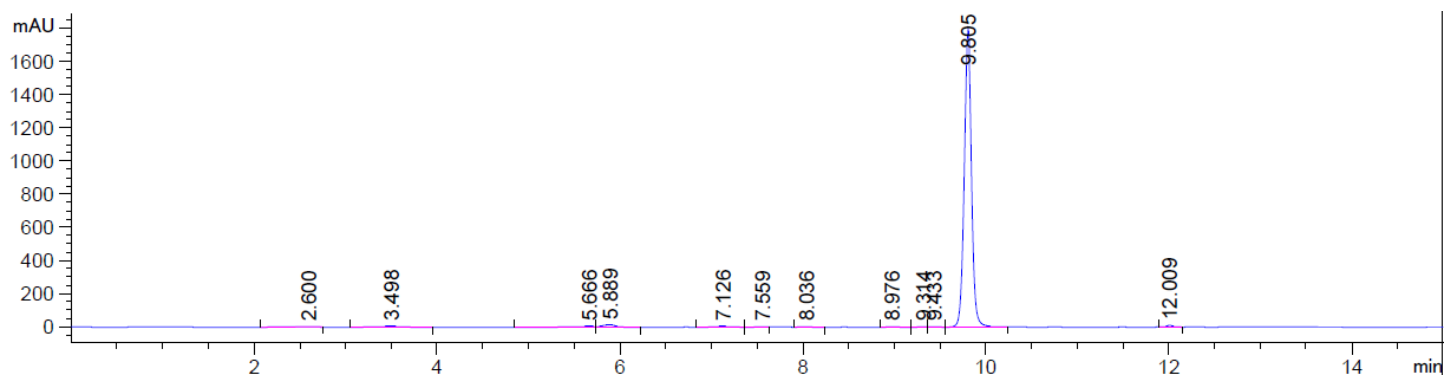

12

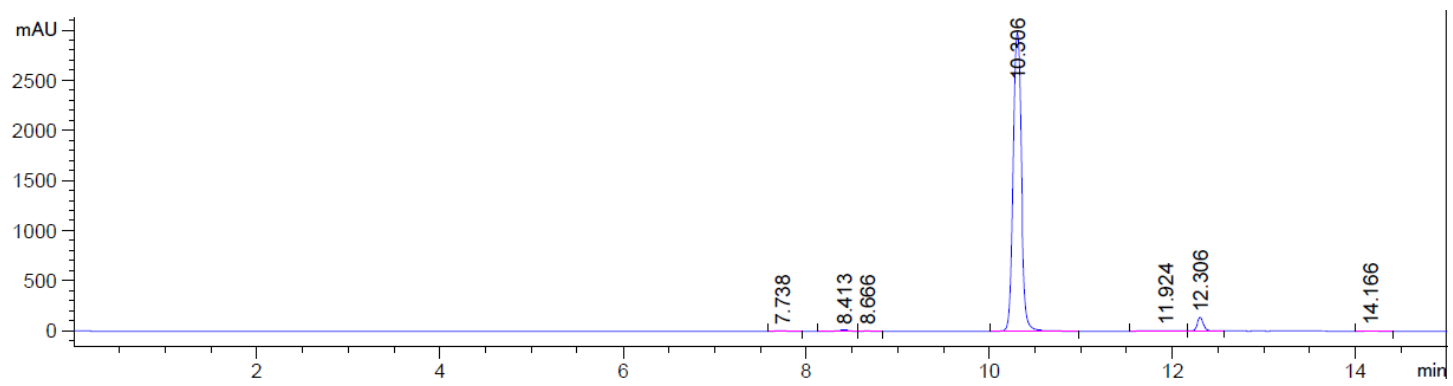

13

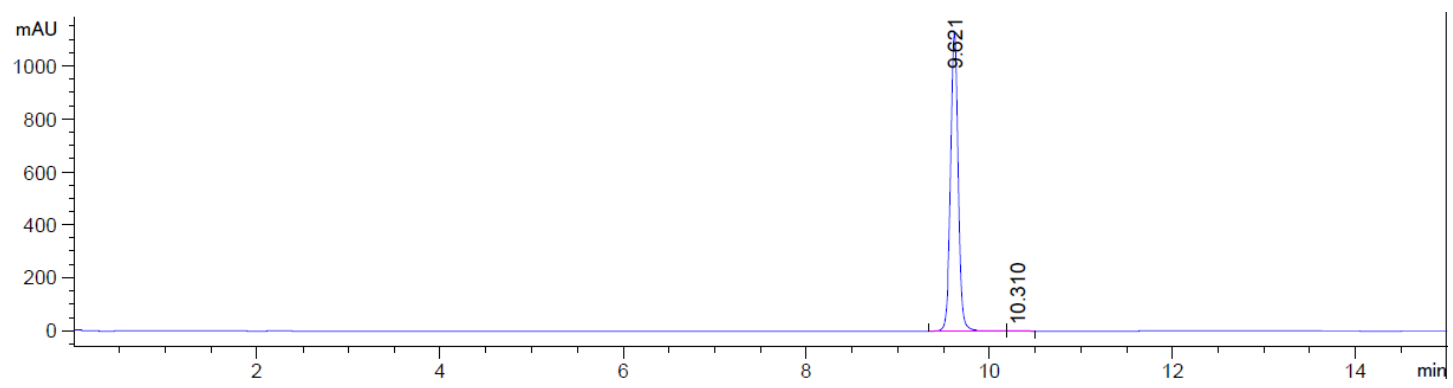

14

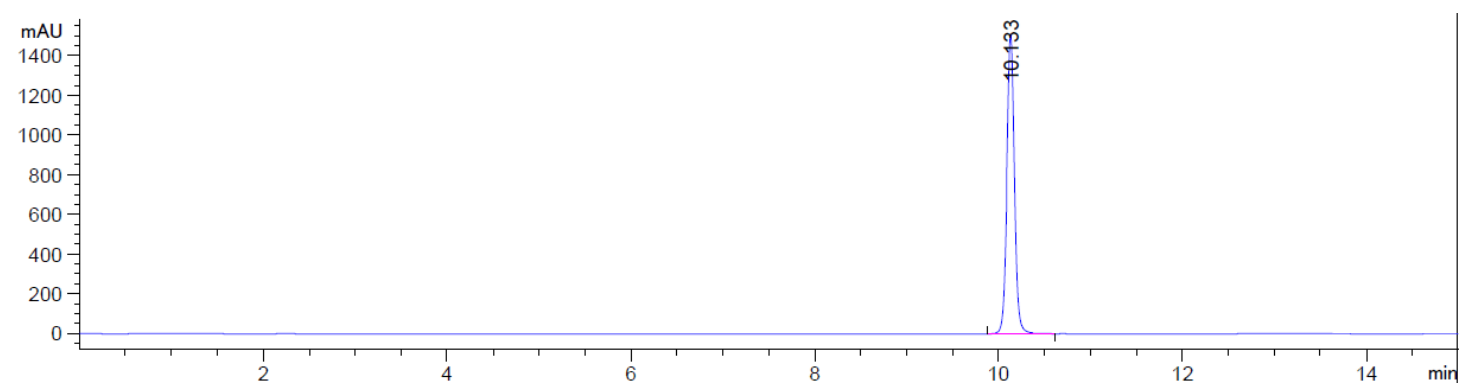

15

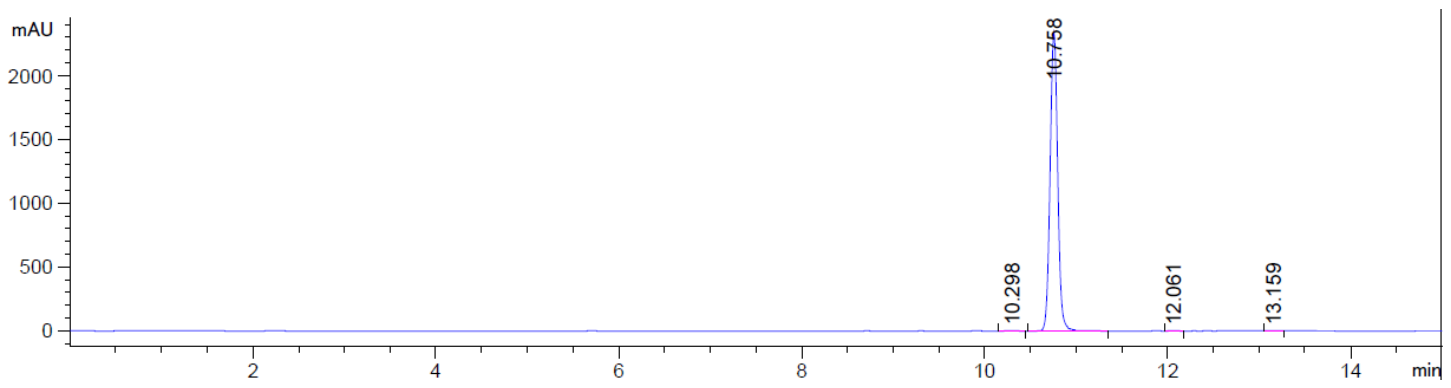




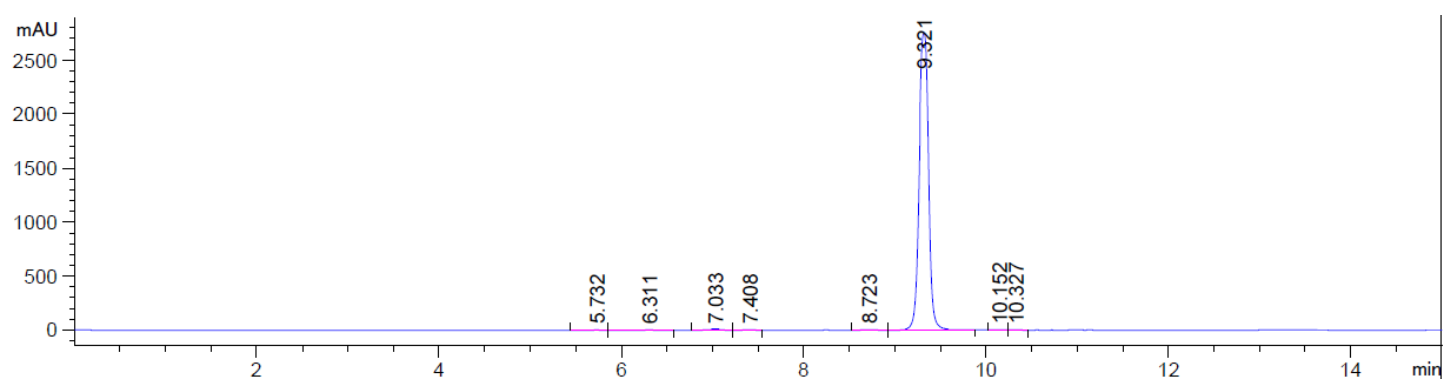

17

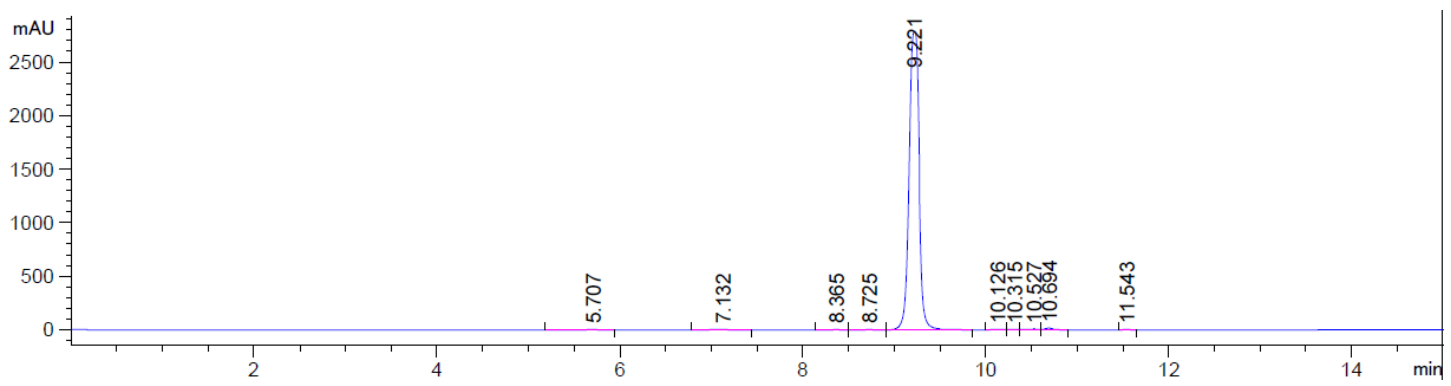

18

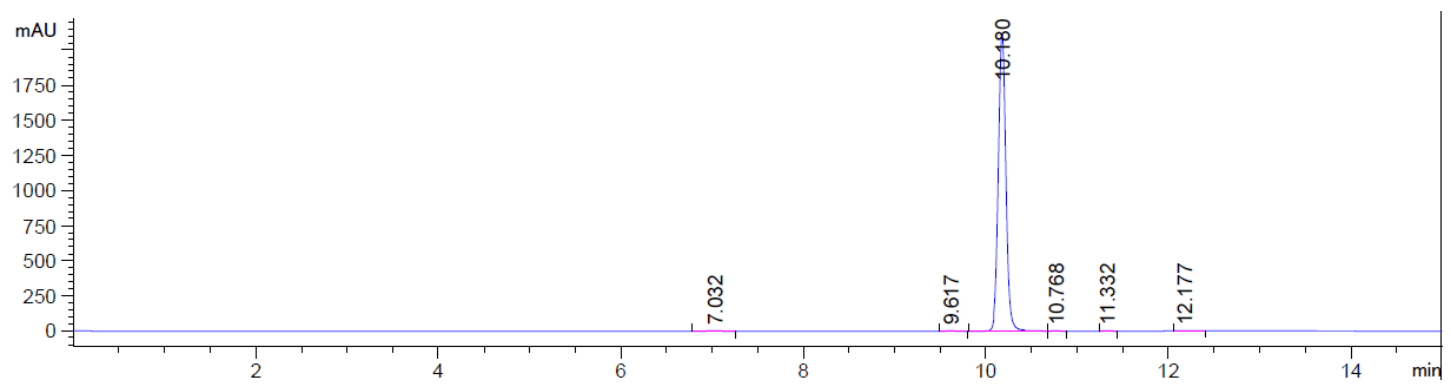

19

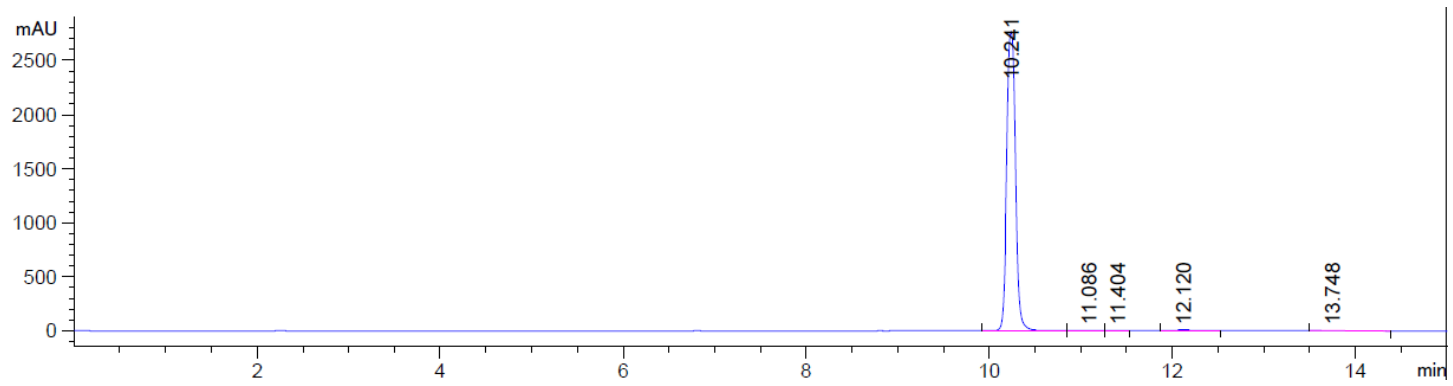

20

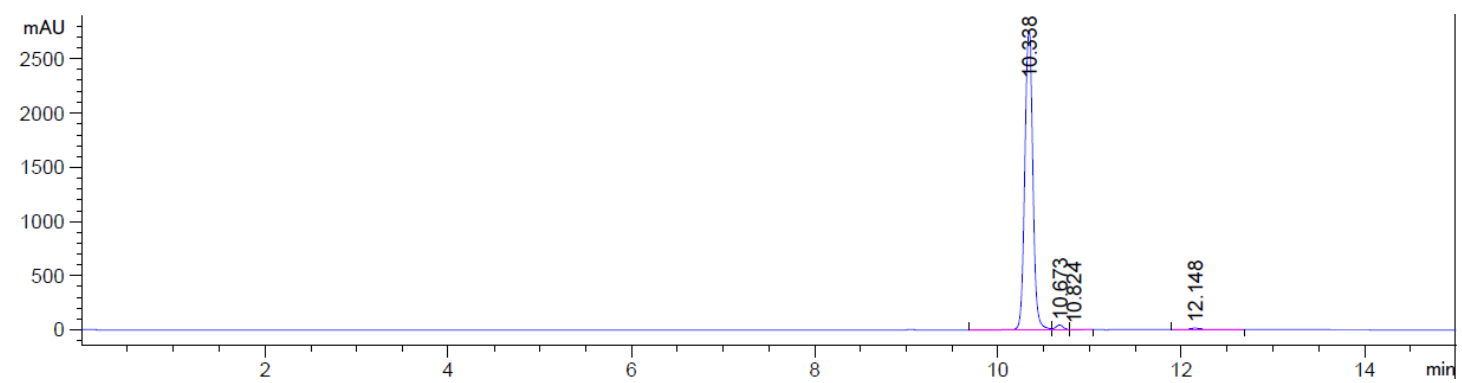

21

21-Apr-2020
20200419-wct-1200-1-1

20200419-wct-1200-1-1

-

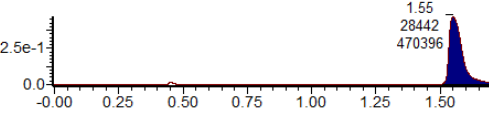

\begin{tabular}{rrr}
2.04 & 2.29 & 2.45 \\
269 & 590 \\
6001 & 23087 & 68 \\
\hline
\end{tabular}

$15: 48: 0$
(1) PDA Ch1 254nm@1.2nn

Area, Heiah
ime Height

$\begin{array}{rrrr}1.55 & 470396 & 28441.77 & 95.8 \\ 2.04 & 6001 & 269.00 & 0.9\end{array}$ 
22

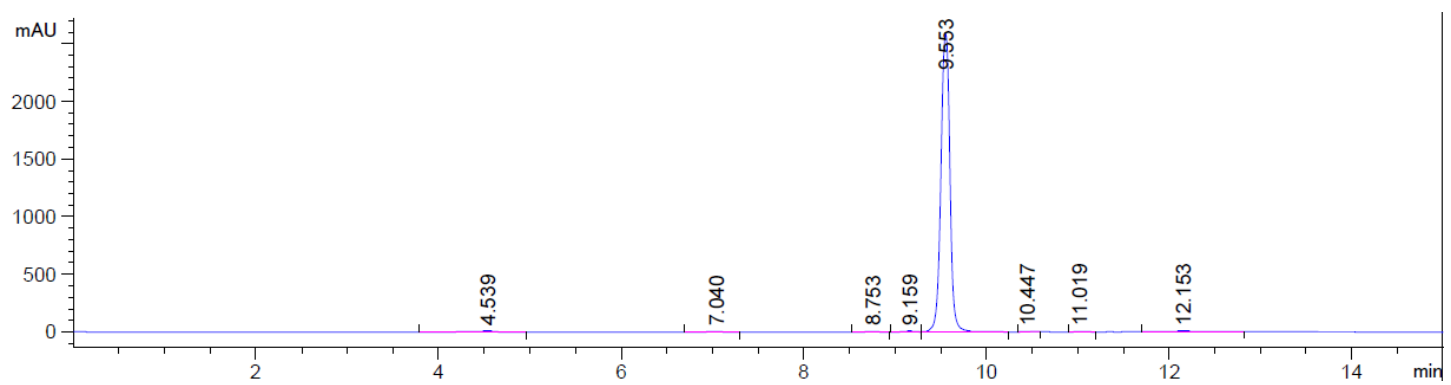

23

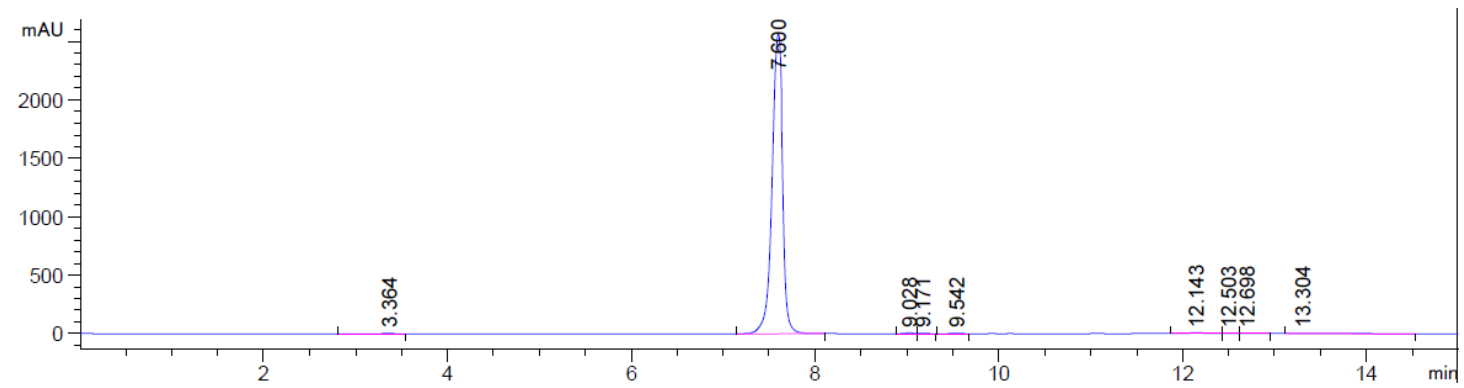

24

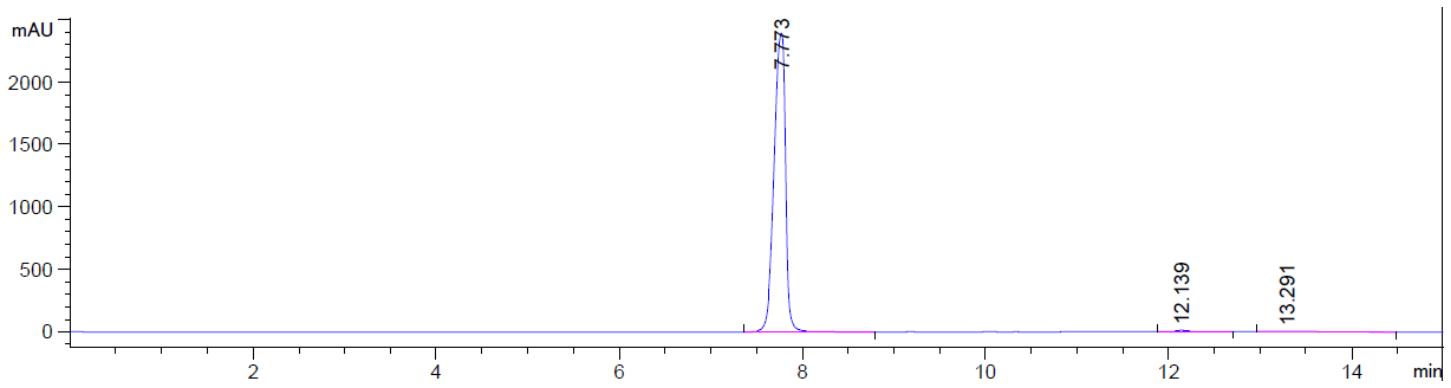

25

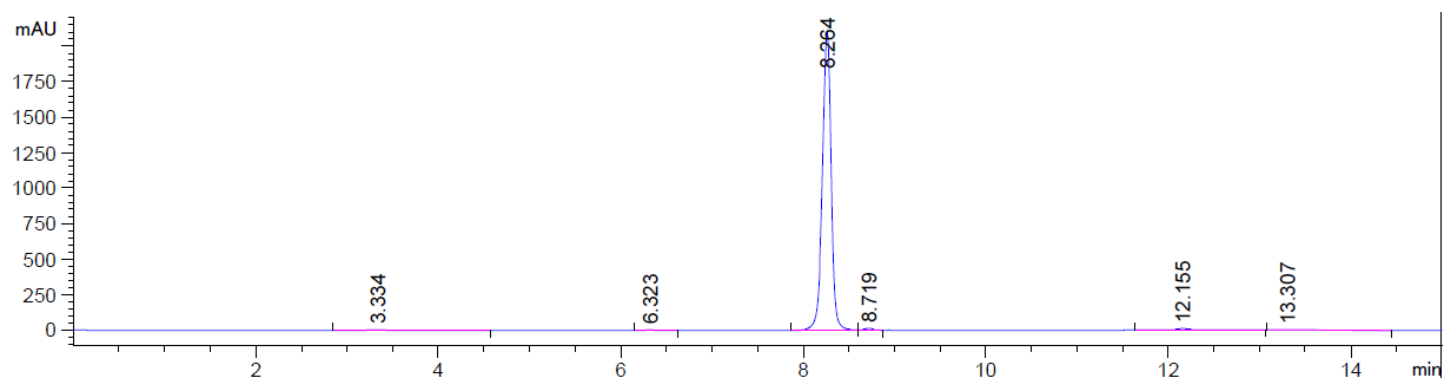

26

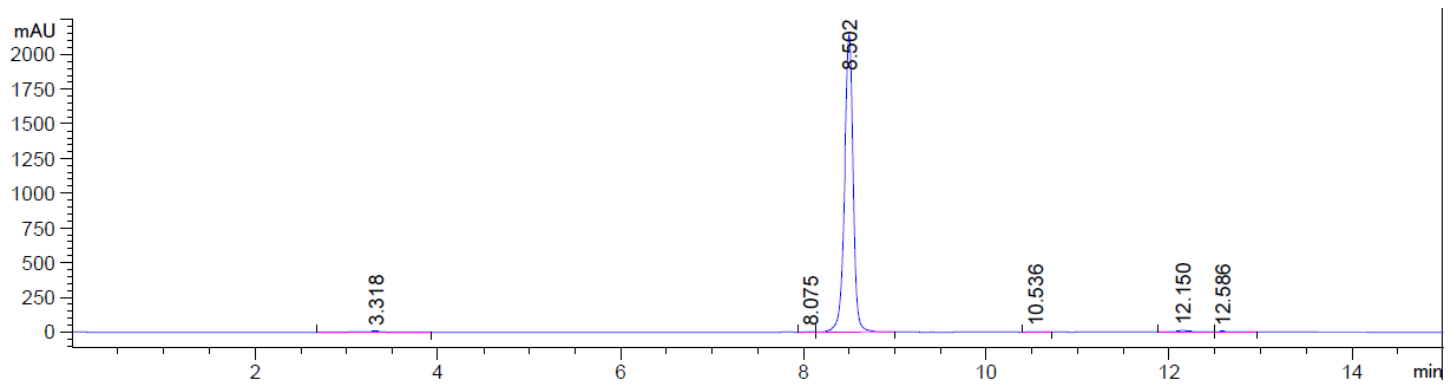


27

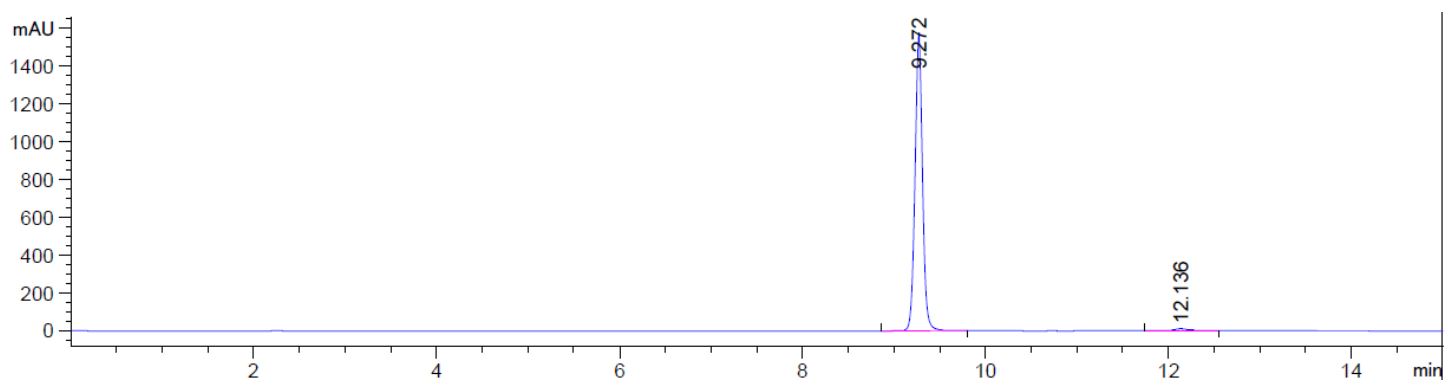

28

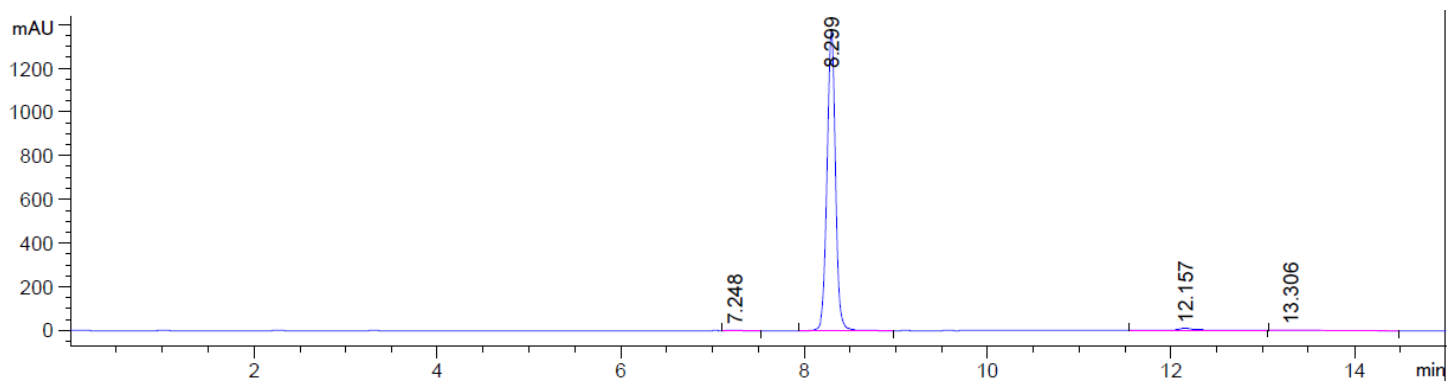

29

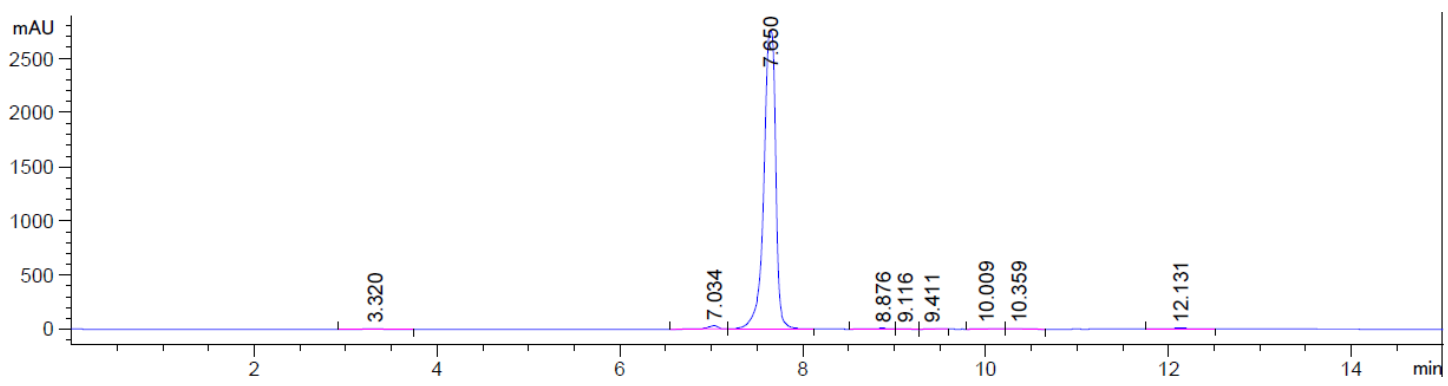

30

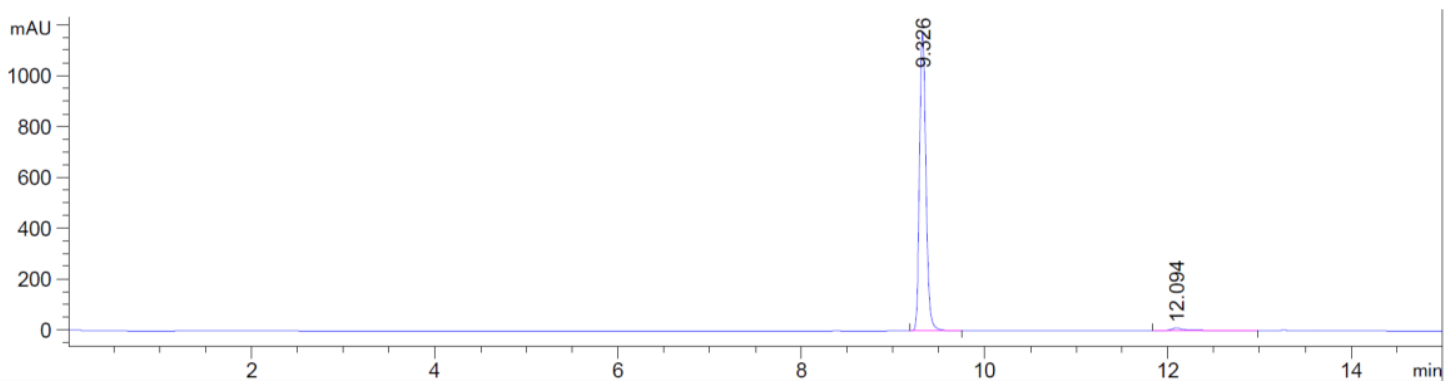

31

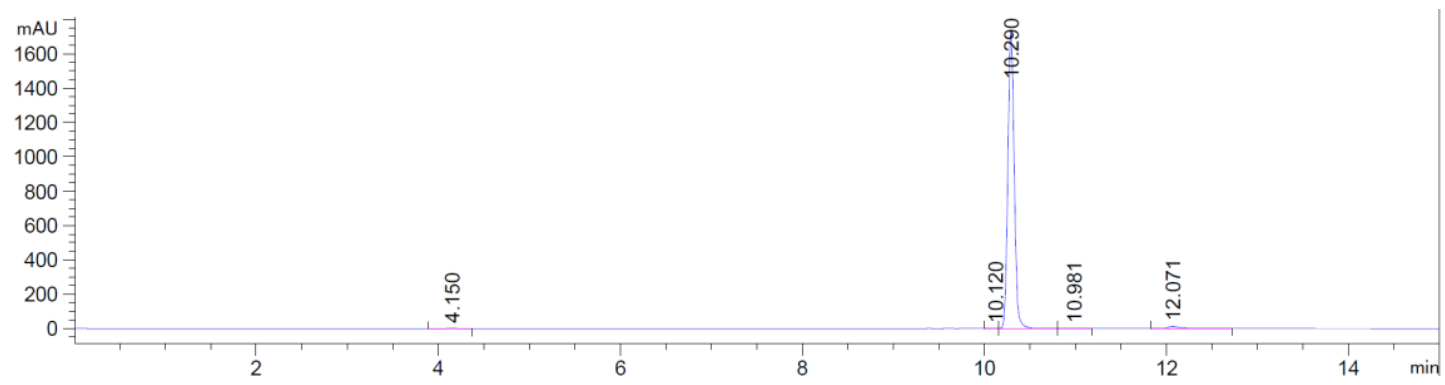


32

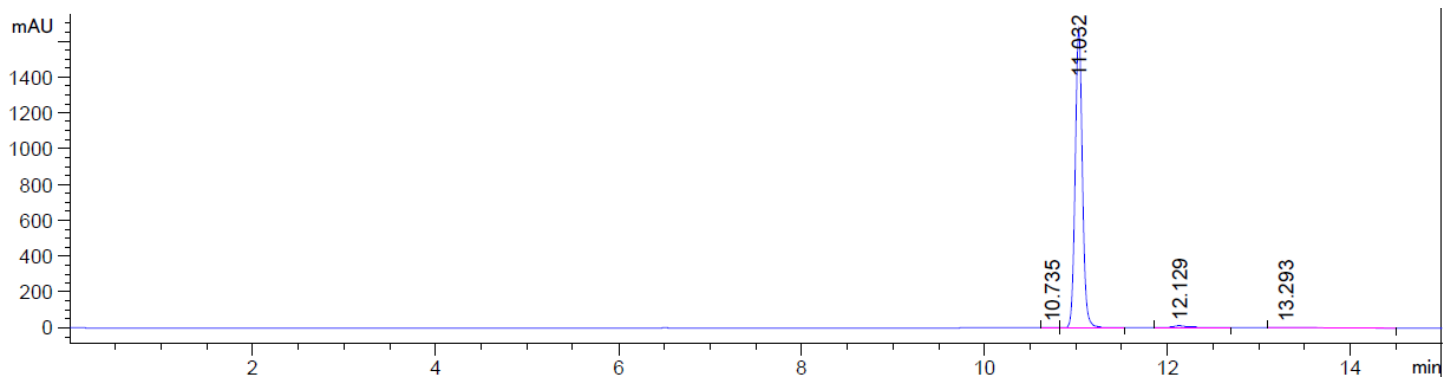

33

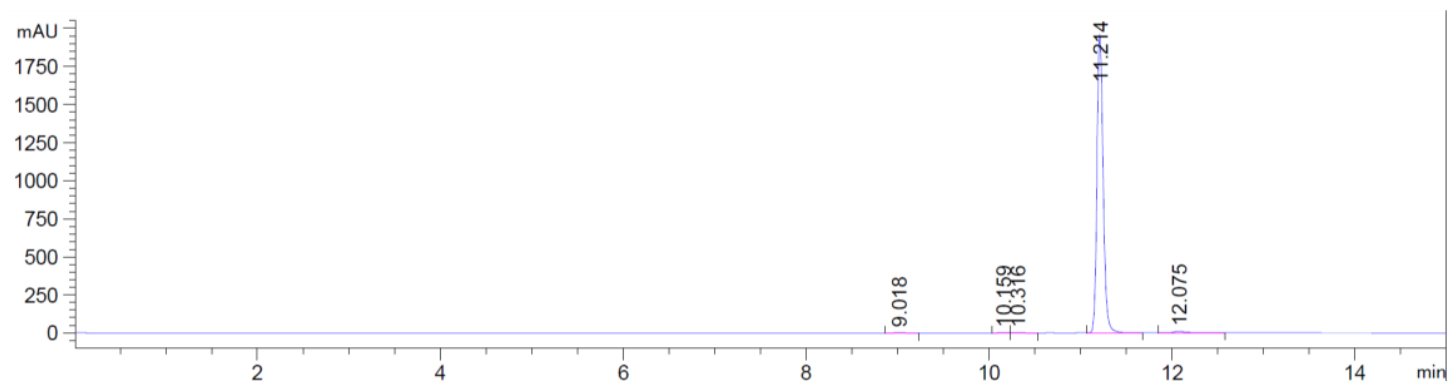

34

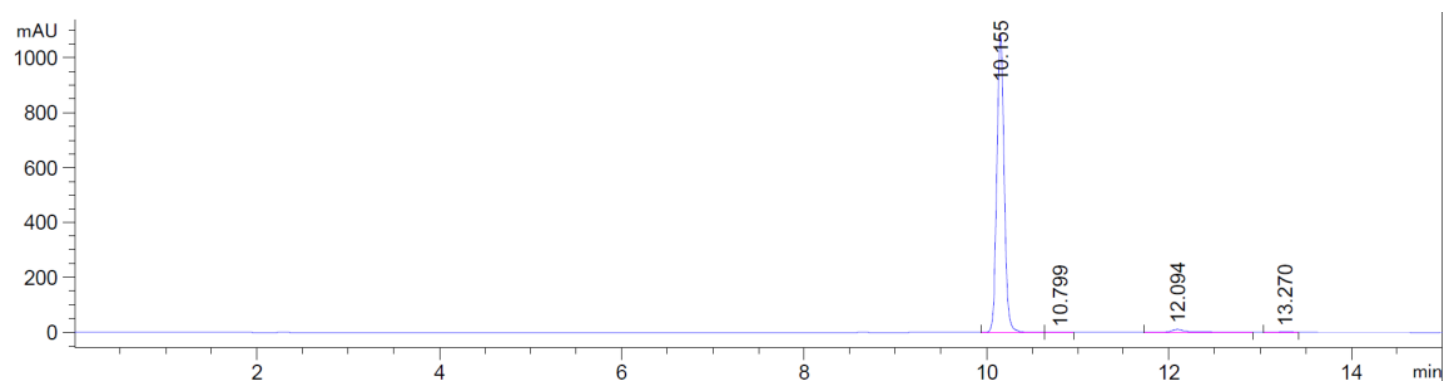

35

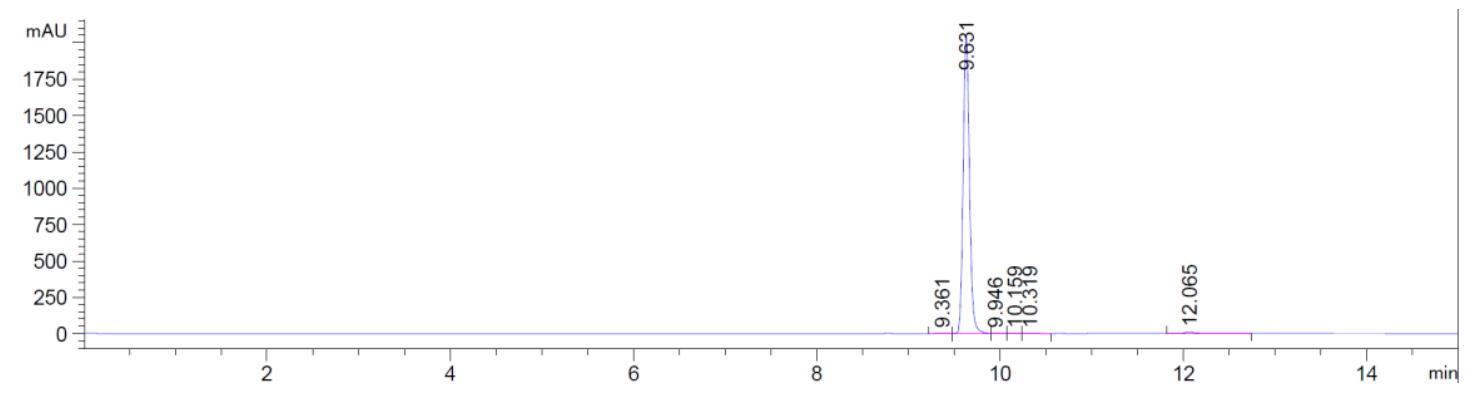

36

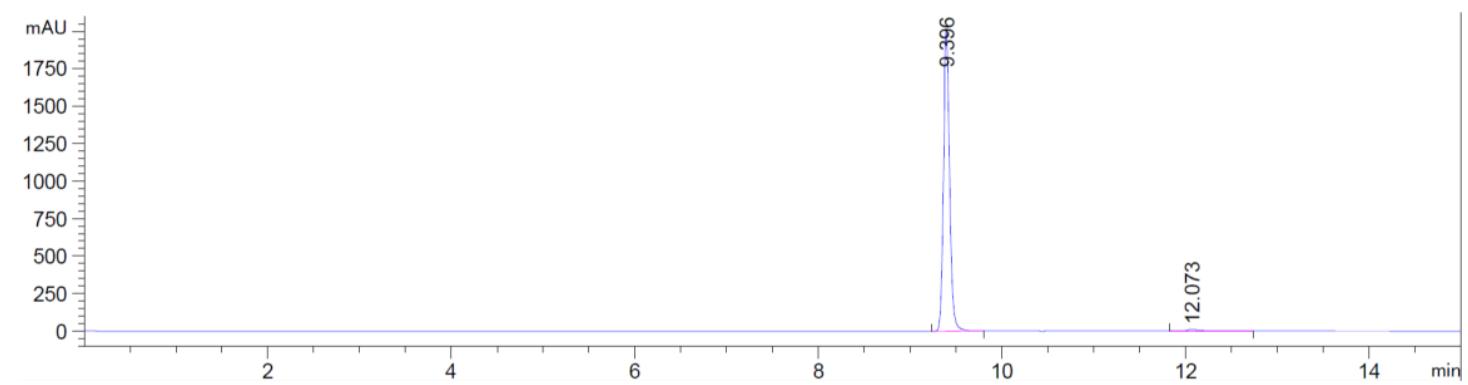


37

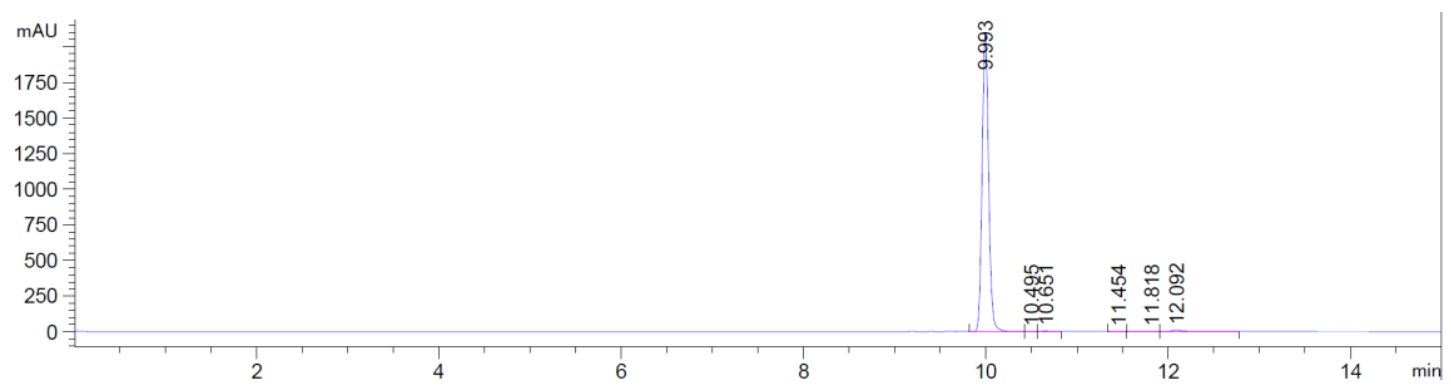

38

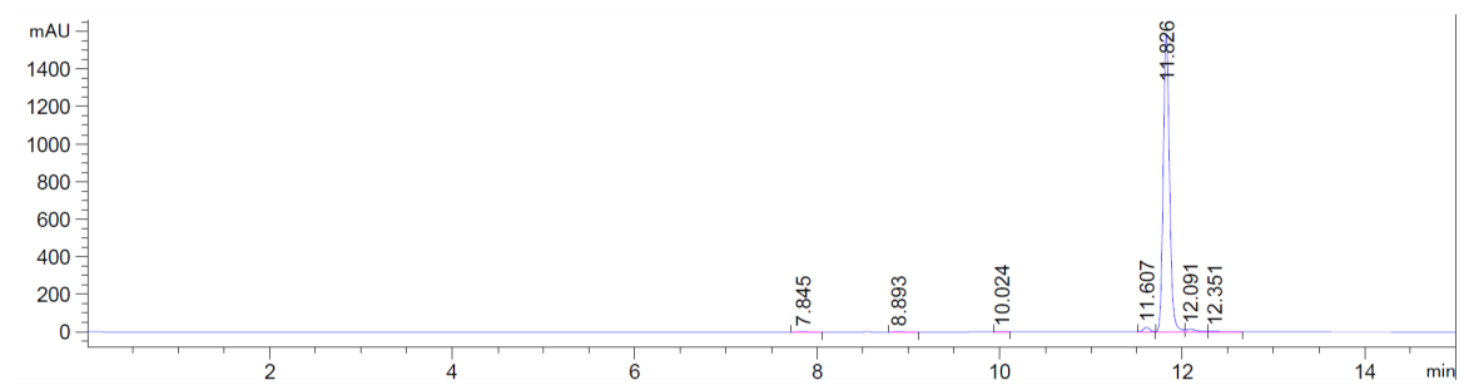

39

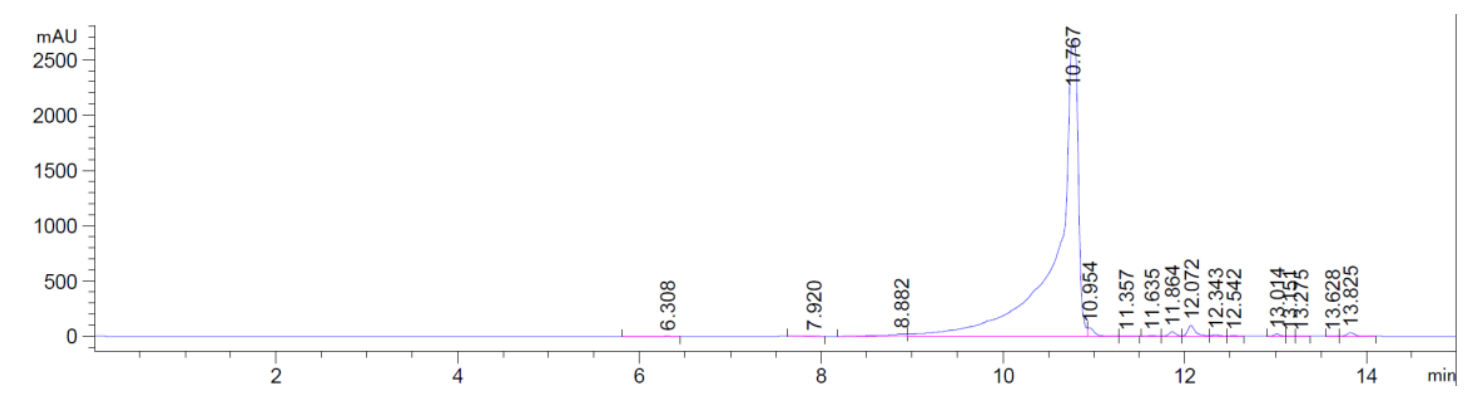

40

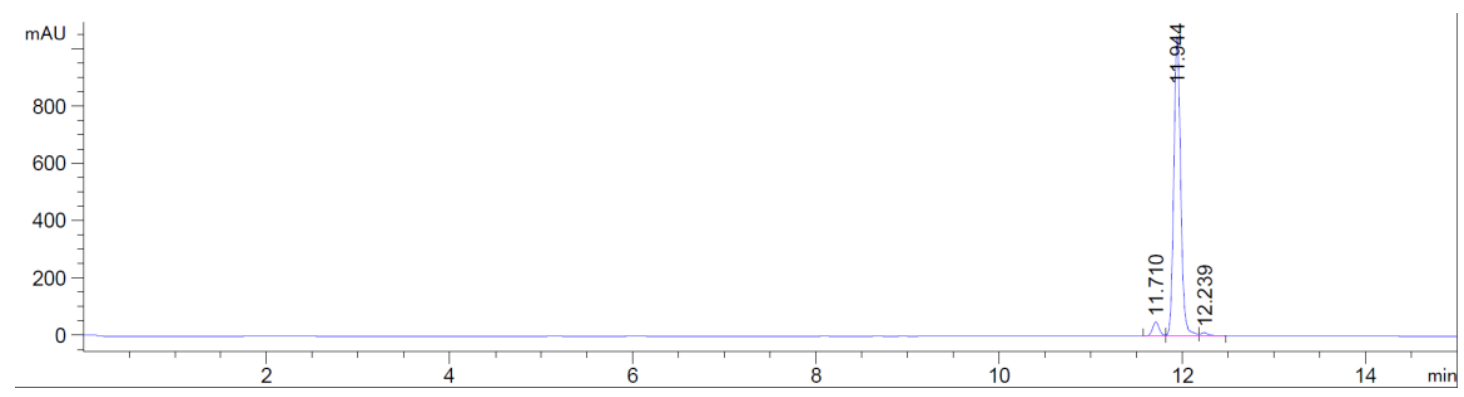

41

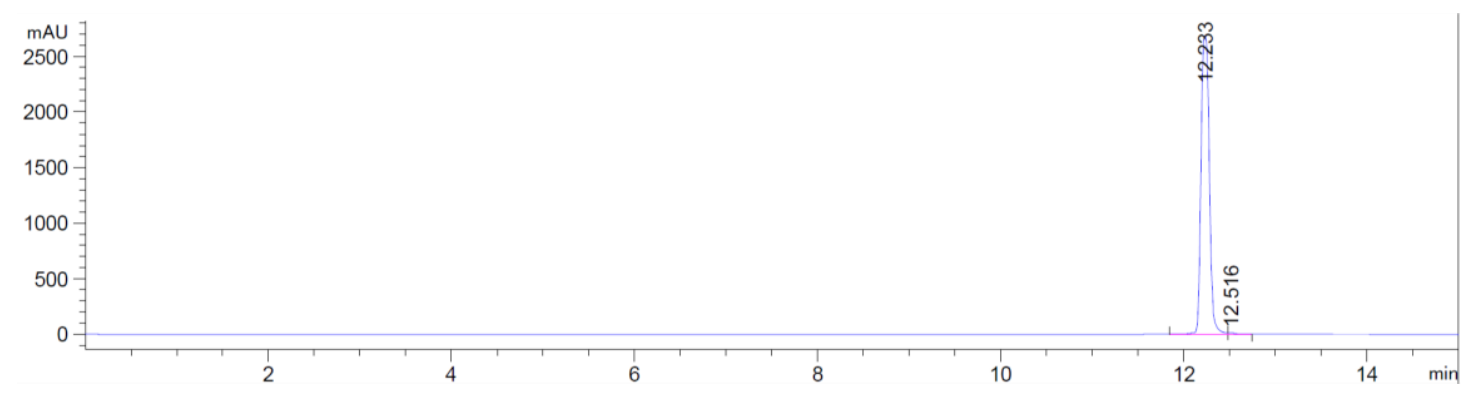


42

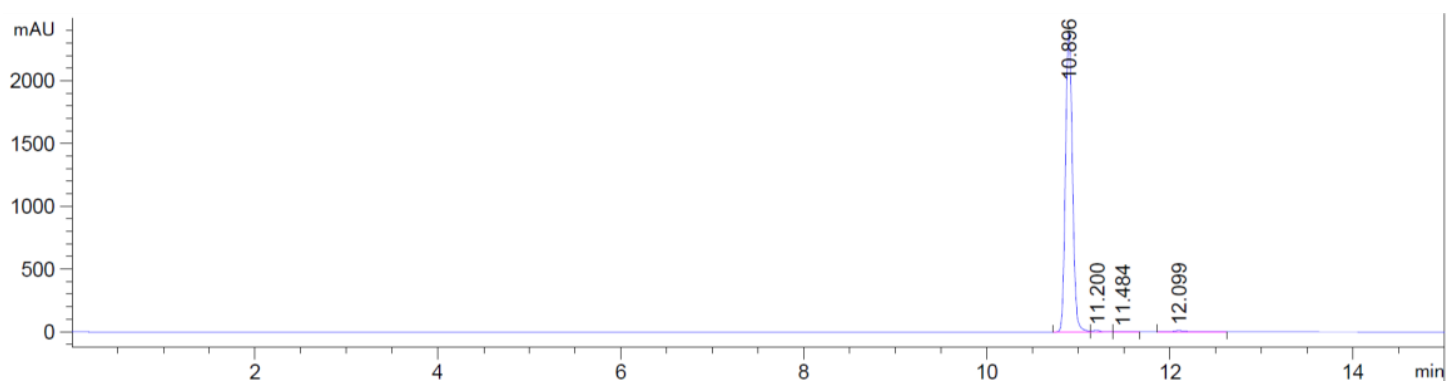

43

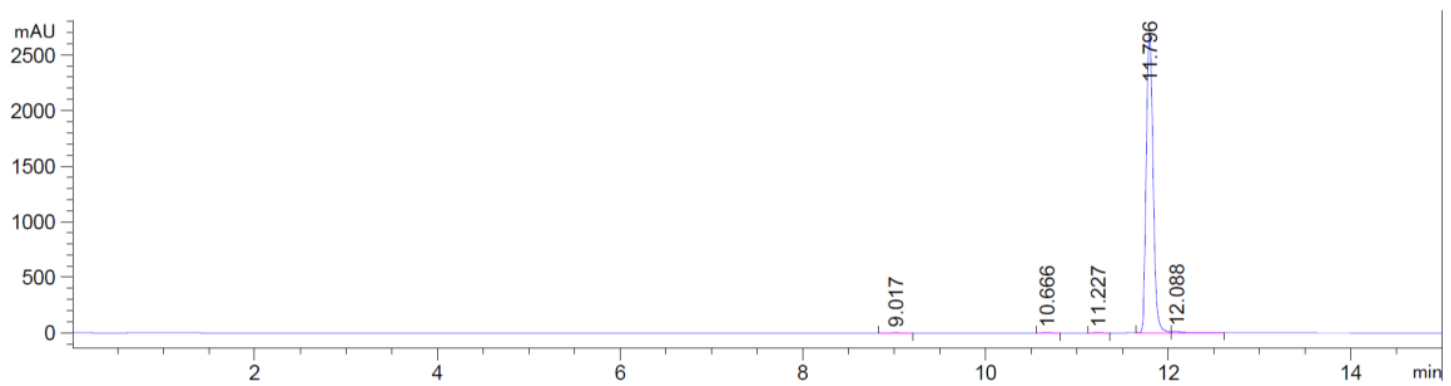

44

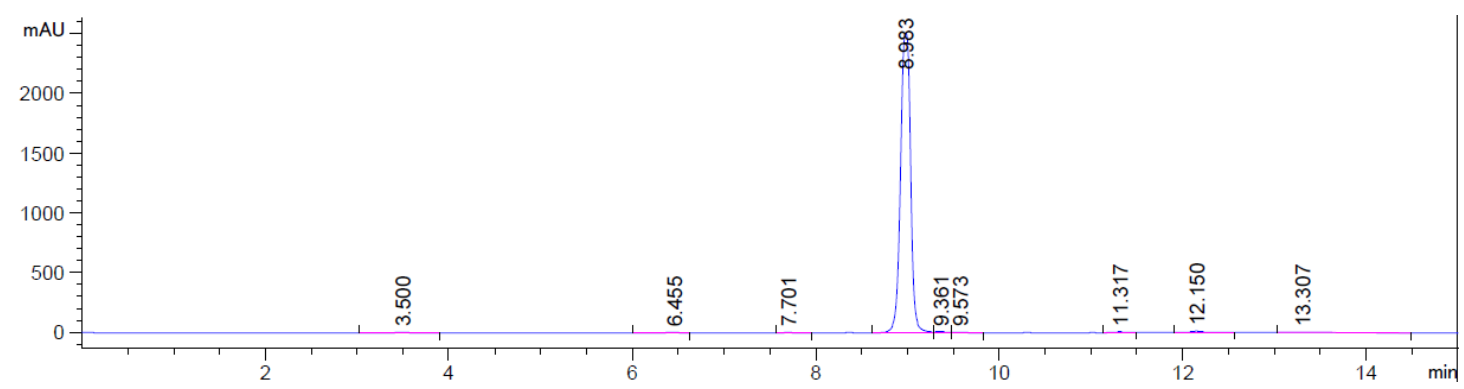

45

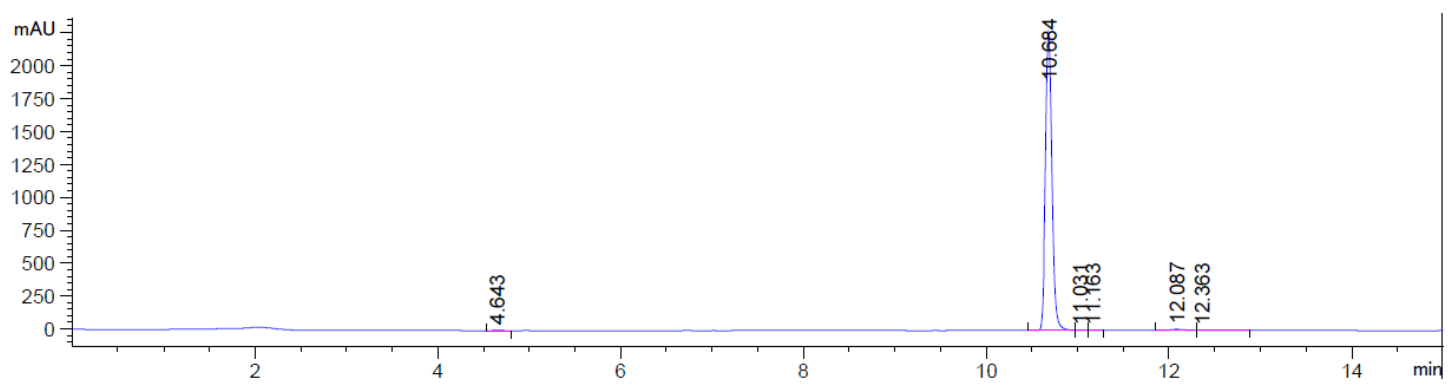

46

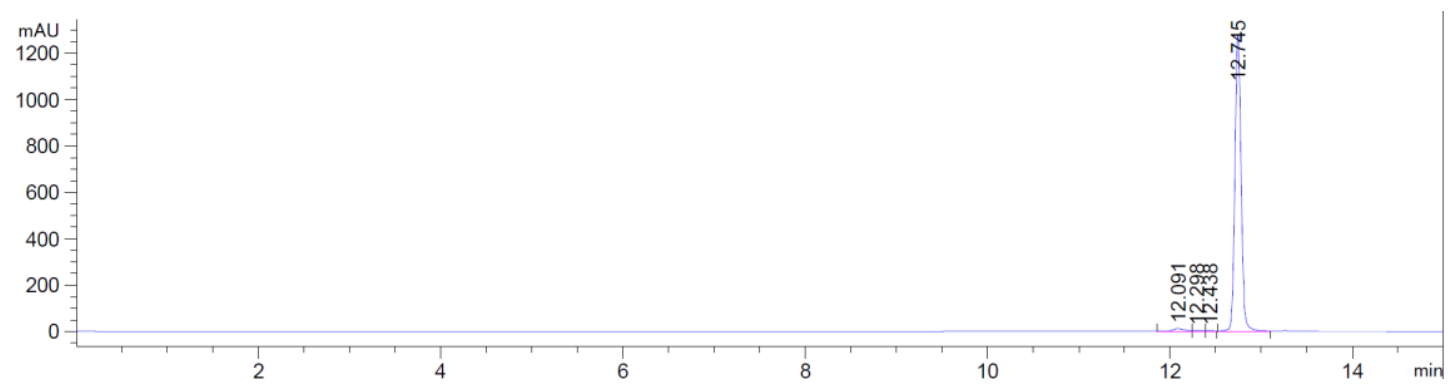


47

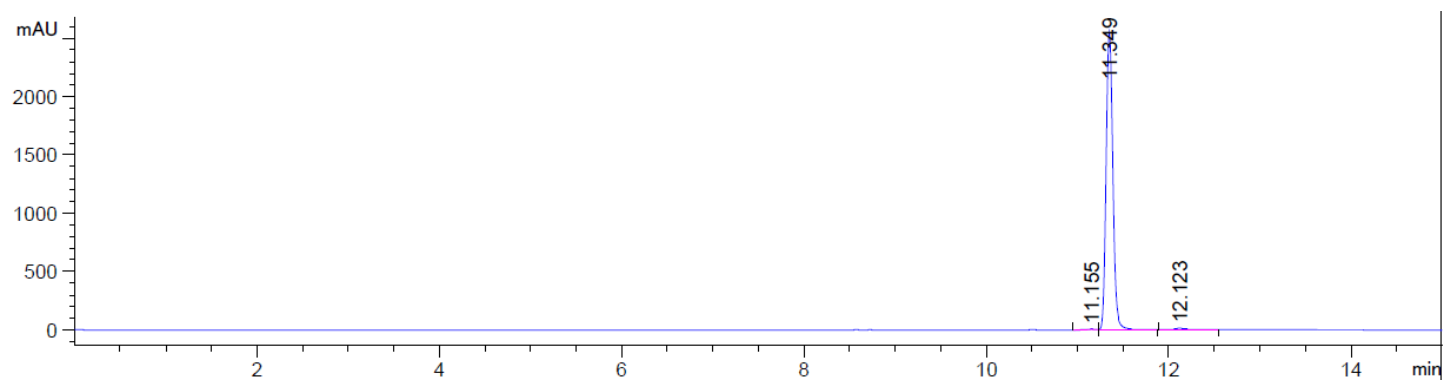

49

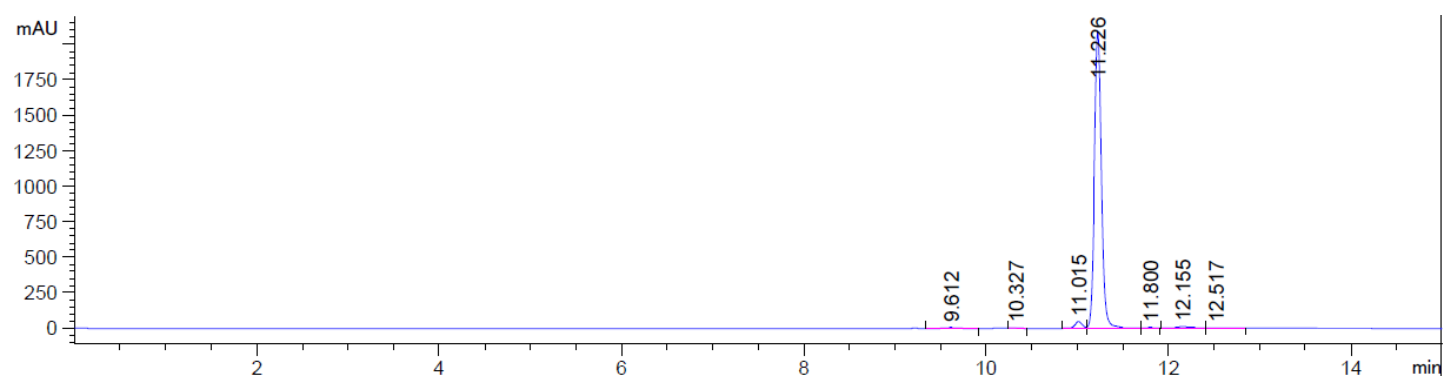

50

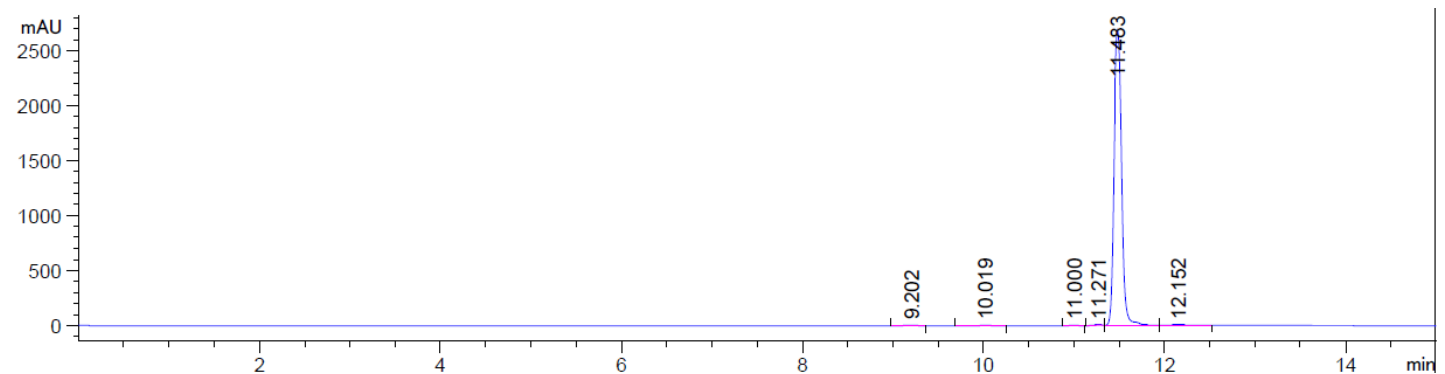

51

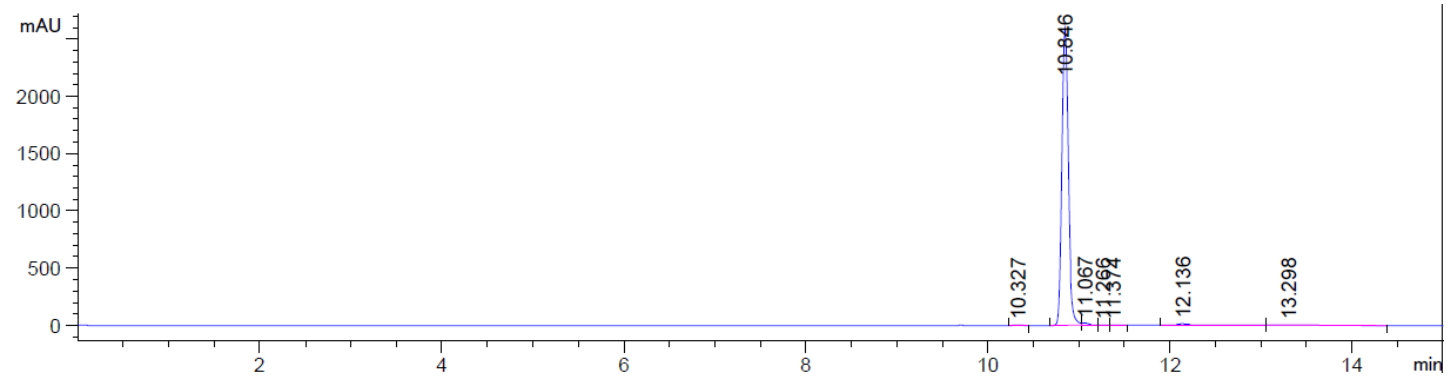

52

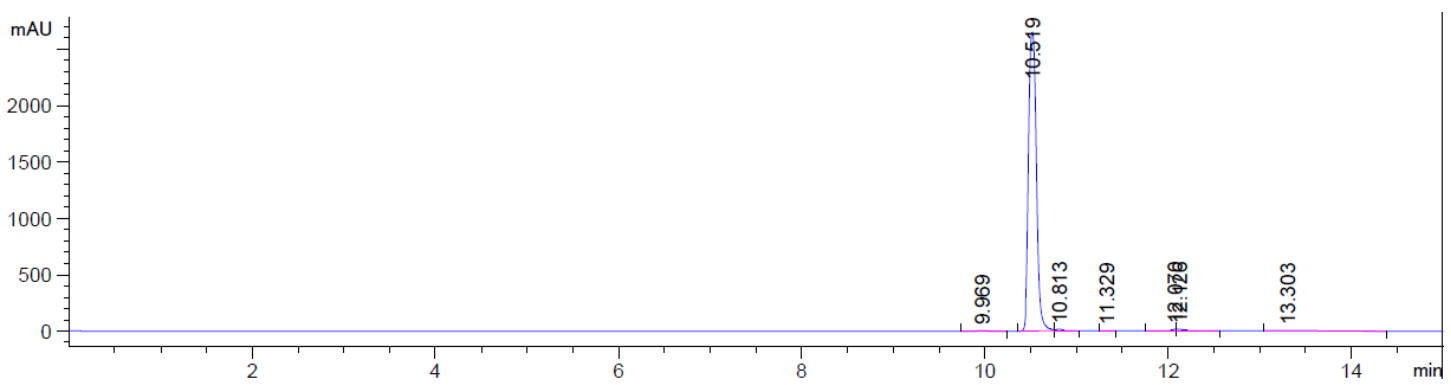


53

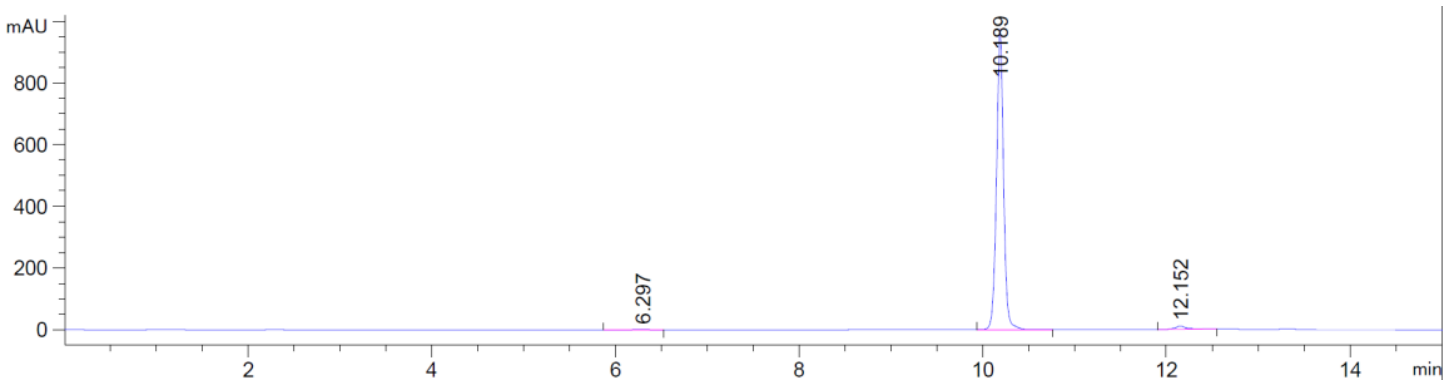

54 (containing an diastereomer as indicated in the ${ }^{1} \mathrm{H}$ NMR)

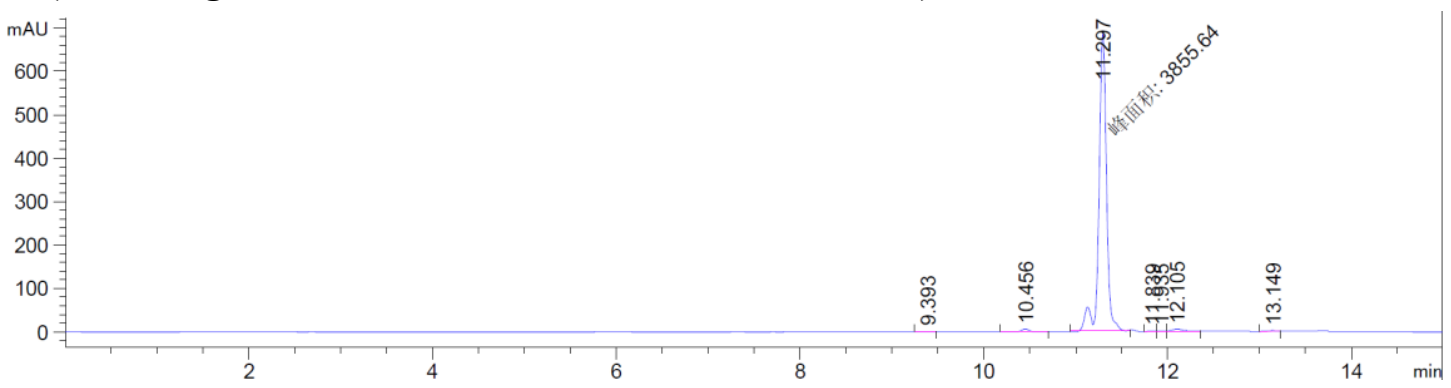

55

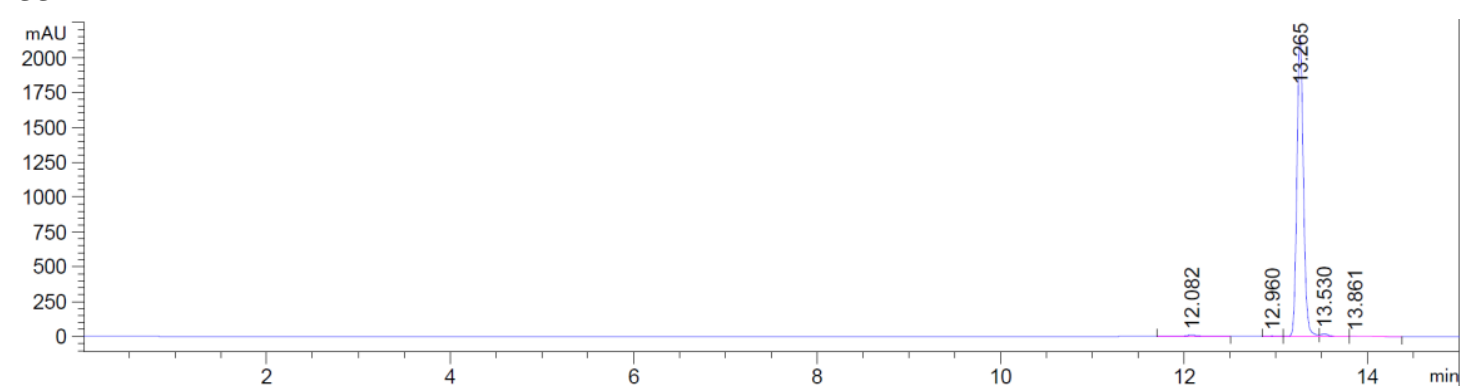

56

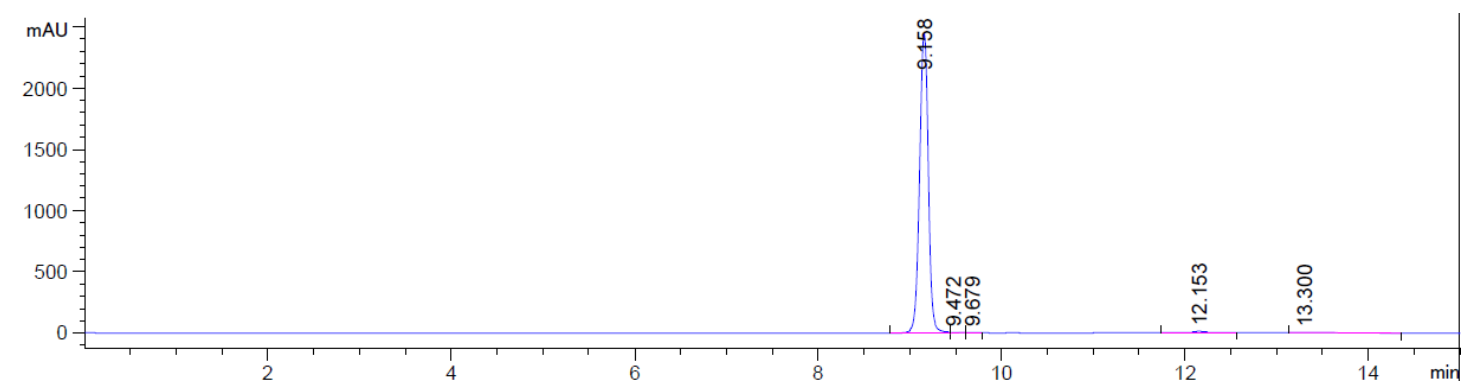

57

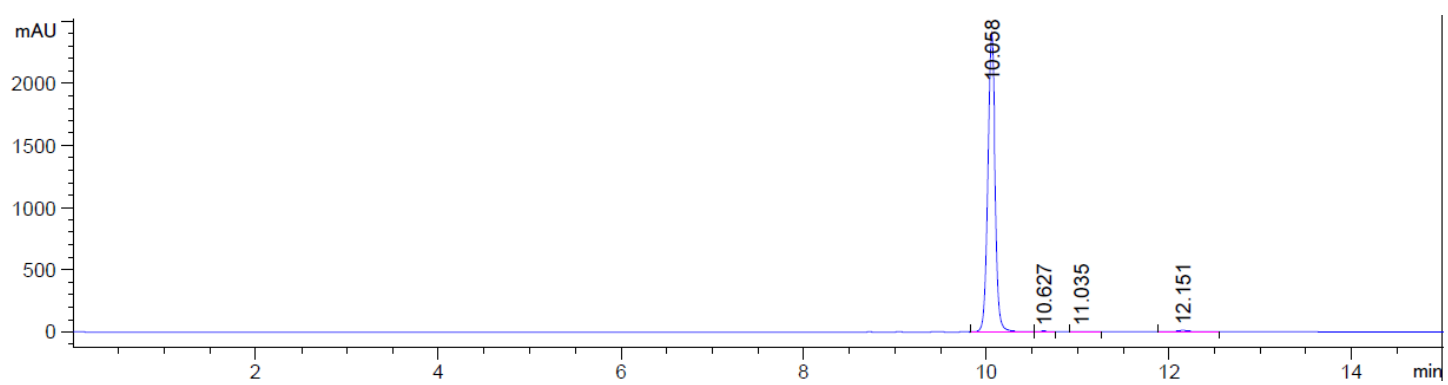


58

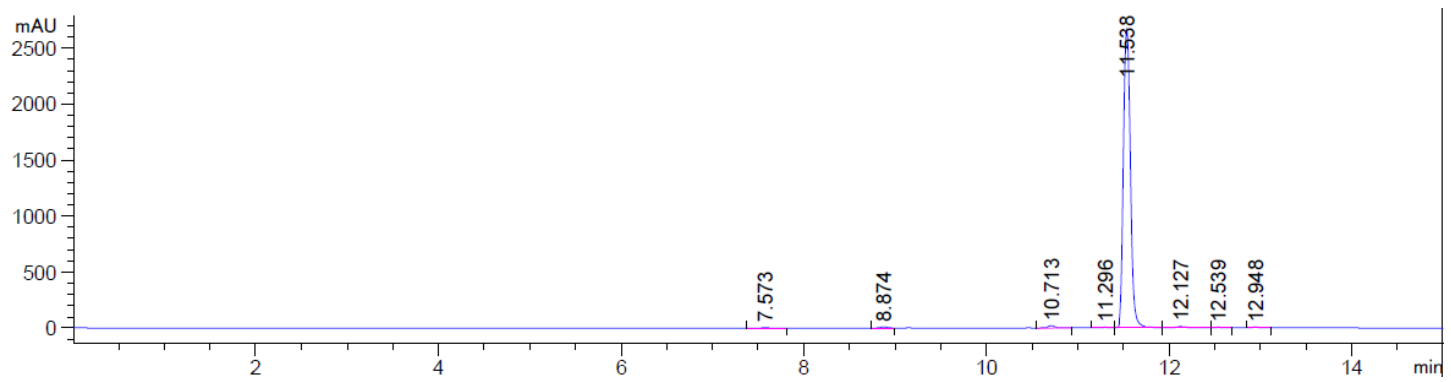

59

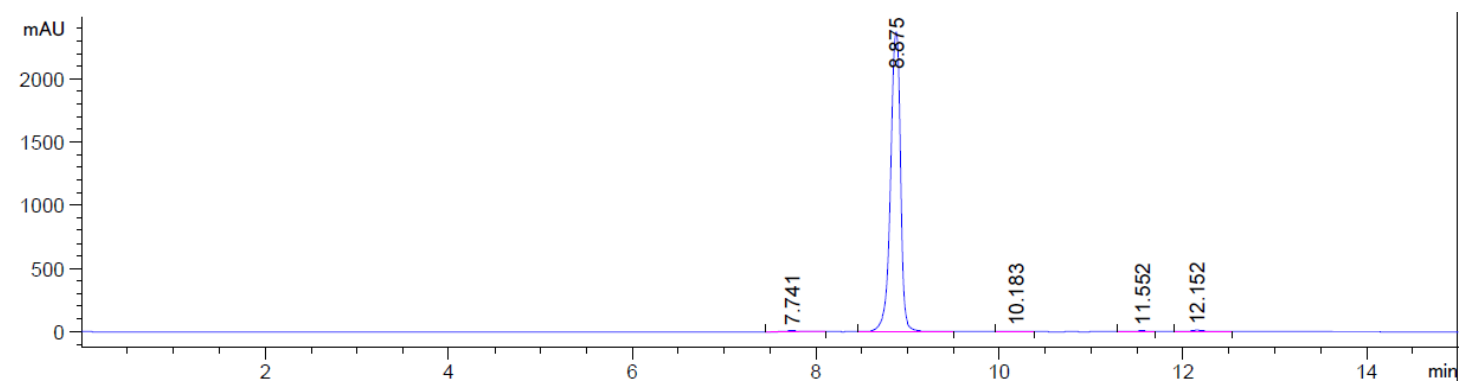

60

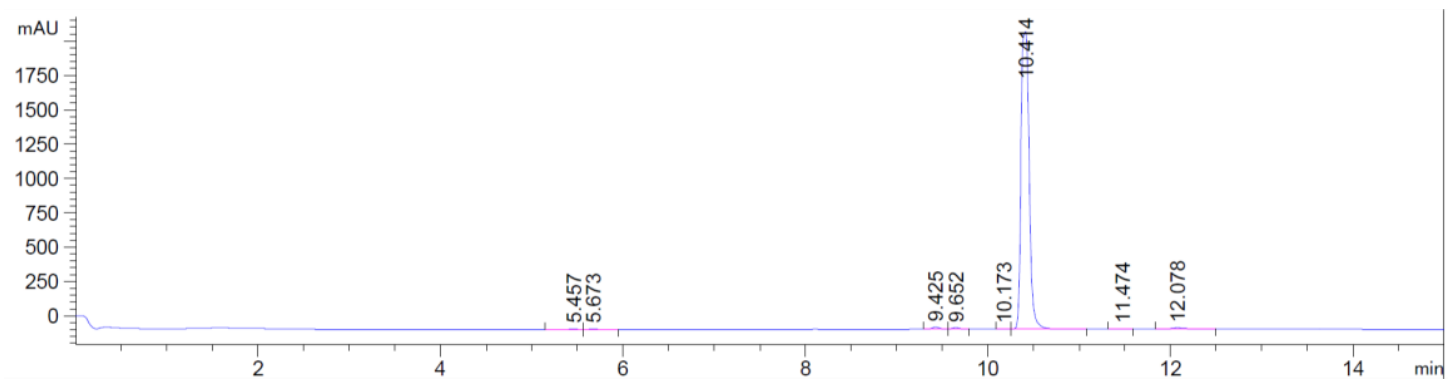

67

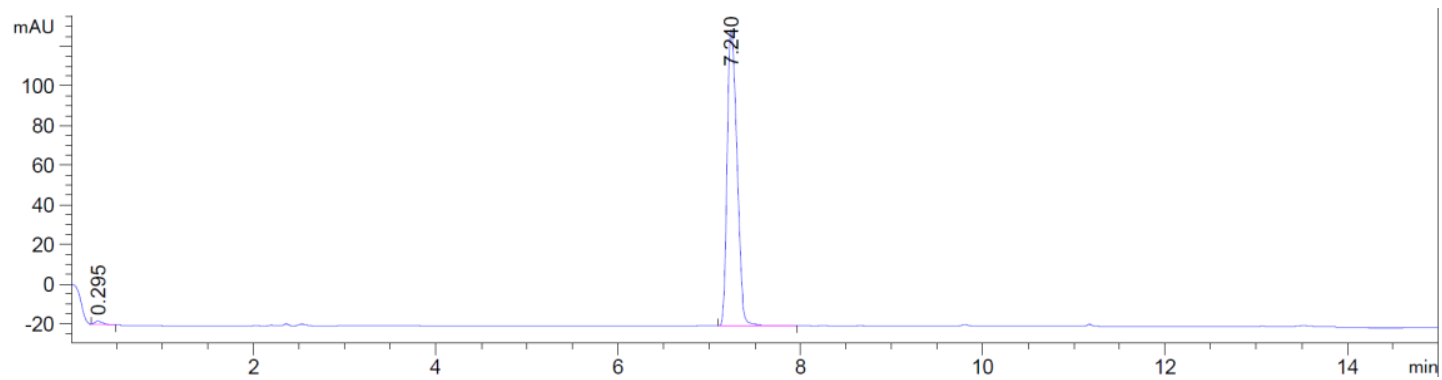

